\title{
Diet, Nutrient Status and - Gestational Diabetes
}

$\therefore$

$8^{80}$

8 Moniek Looman

○

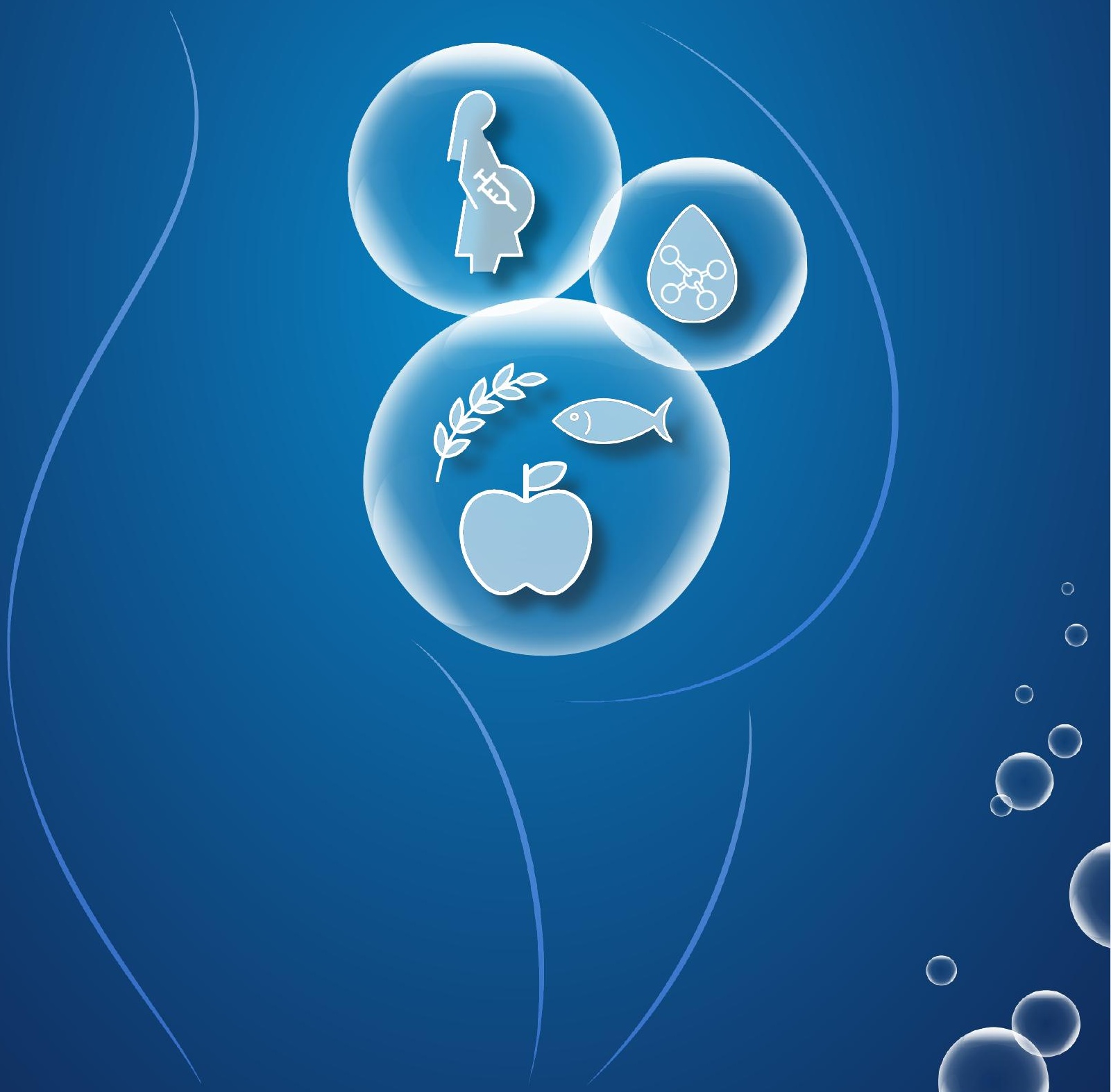




\section{Diet, nutrient status and gestational diabetes}

Moniek Looman 


\section{Thesis committee}

\section{Promotor}

Prof. Dr E.J.M. Feskens

Professor of Nutrition and Health over the Lifecourse

Wageningen University \& Research

\section{Co-promotor}

Dr A. Geelen

Associate professor at the Division of Human Nutrition

Wageningen University \& Research

\section{Other members}

Prof. Dr B. Kemp, Wageningen University \& Research

Prof. Dr T.J. Roseboom, AMC - University of Amsterdam

Dr H.W. de Valk, UMC Utrecht

Dr M. Erkkola, University of Helsinki, Finland

This research was conducted under the auspices of the Graduate School VLAG (Advanced studies in Food Technology, Agrobiotechnology, Nutrition and Health Sciences). 


\title{
Diet, nutrient status and gestational diabetes
}

\author{
Moniek Looman
}

Thesis

submitted in fulfilment of the requirements for the degree of doctor

at Wageningen University

by the authority of the Rector Magnificus,

Prof. Dr A.P.J. Mol,

in the presence of the

Thesis Committee appointed by the Academic Board

to be defended in public

on Friday 18 May 2018

at 1.30 p.m. in the Aula. 


\section{Moniek Looman}

Diet, nutrient status and gestational diabetes 220 pages

PhD thesis, Wageningen University, Wageningen, the Netherlands (2018)

With references, with summary in English

ISBN: 978-94-6343-863-6

DOI: $\quad 10.18174 / 446742$ 
Table of contents

$\begin{array}{lll}\text { Chapter } 1 & \text { General introduction } & 7\end{array}$

Chapter 2 Development and evaluation of the Dutch Healthy Diet index $2015 \quad 27$

Chapter 3 Using enhanced regression calibration to combine dietary intake

51 estimates from 24-hour recall and food frequency questionnaire improves diet-disease associations

Chapter 4 Adverse pregnancy outcomes in treated gestational diabetes and 69 untreated borderline gestational diabetes

Chapter 5 Pre-pregnancy dietary carbohydrate quantity and quality, and risk 85 of developing gestational diabetes: the Australian Longitudinal Study on Women's Health

Chapter 6 Pre-pregnancy dietary micronutrient adequacy and risk of developing gestational diabetes: the Australian Longitudinal Study on Women's Health

Chapter 7 Longitudinal study on micronutrient intake and status, diet quality and glucose tolerance from preconception to the second trimester of pregnancy

Chapter 8 Supplement use and dietary sources of folate, vitamin D, and n-3 fatty acids during preconception: the GLIMP2 study

Chapter 9 General discussion

Samenvatting

Dankwoord 



\section{Chapter 1}

General introduction 


\section{The role of diet in pregnancy}

An adequate and healthy diet is needed to ensure optimal foetal development, birth outcomes and health later in life [1-3]. The maternal diet must provide sufficient energy, macro- and micronutrients to meet the mother's usual requirements, as well as the needs of the growing foetus, and supporting tissues [4]. Undernutrition during pregnancy can have lasting negative consequences for the offspring's health and in this way, many chronic diseases including cardiovascular disease and diabetes may originate in the womb [5]. The hypothesis for the underlying mechanism of these associations is called foetal programming and was first introduced by Hales and Barker in their developmental origins of health and disease (DOHaD) hypothesis [6]. It assumes that metabolic changes in the mother during pregnancy can lead to structural and functional adaptations during the development of the foetus, with potential consequences for growth and metabolism in the child's later life.

Historically, the DOHaD hypothesis focused on the effects of maternal undernutrition. Nowadays, besides maternal undernutrition, maternal overnutrition poses a major public health challenge [7]. Maternal obesity has been associated with both intrauterine growth restriction and large-forgestational age foetuses and is contributing to the epidemic of childhood obesity and metabolic syndrome [8].

Dietary recommendations for pregnant women are quite similar to those for other adults with the main recommendation to follow a healthy and balanced diet [9-13]. Most national guidelines, including Dutch guidelines, recommend an additional energy intake of 150-500 kcal/d in the second and third trimester. Although most pregnant women did not meet this recommendation [14, 15], it is unlikely that women are in energy deficit during pregnancy, despite energy intakes below recommendations, as there is an increasing prevalence of overweight and excessive gestational weight gain during pregnancy in developed countries [16-18]. A plausible reason for the observed discrepancy could be that pregnant women compensate for the increased energy need by reducing their physical activities [19].

Adequate micronutrient intake is critical for foetal development. Micronutrients may affect pregnancy outcomes and foetal development through alterations in maternal and foetal metabolism owing to their role in enzyme activity, signal transduction and transcription pathways, biological functions and oxidative stress [2]. However, the biological mechanisms underlying these associations are not completely understood. The most well-known example is that adequate folate intake in the periconceptional period reduces the risk of neural tube defects [20]. Furthermore, methyl-donor nutrients such as folate, vitamin B6 and vitamin B12 have been positively associated with brain growth and cognitive development in the offspring [21, 22]. In addition, zinc, iron and n-3 fatty acids have 
been related to foetal brain development, as deficiencies were associated with higher risk of preterm birth and poorer attention, learning and memory [23-25]. Sufficient iron intake is also needed to accommodate the increased production of haemoglobin, as blood volume expands by approximately $1500 \mathrm{ml}$, with a 200-250 $\mathrm{ml}$ increase in red blood cell mass during pregnancy [26]. Vitamin D requirements are increased to support foetal skeletal growth, and vitamin $D$ deficiency has also been linked to low birth weight, increased risk of small for gestational age infants [27] and immune function [28]. A meta-analysis examined adherence of pregnant women to micronutrient recommendations in developed countries and observed that most women do not meet micronutrient recommendations. In particular, suboptimal intakes of folate and vitamin D were observed in a large proportion of the pregnant women [29].

\section{Supplement use}

The gap between recommended and actual dietary intake can be partly bridged by taking supplements. The World Health Organization (WHO) recommends folic acid supplements to reduce risk of neural tube defects and iron supplements for pregnant women at risk of developing anaemia [30]. Furthermore, some countries, including the Netherlands, recommend vitamin D supplementation for pregnant women as vitamin D deficiency is common in pregnant populations [26, 31]. However, the WHO advises against other supplements including multivitamins as there has been no proven additional benefit of other supplements [30] and it may even lead to consumption of micronutrients above the recommended upper level of intake [32].

\section{Nutrient status}

Adequate dietary intake is needed to ensure adequate nutrient stores to support the developing foetus without depleting the mother. Intake and status are both part of the DISH model (Figure 1.1), a theoretical model linking behaviour via intake and status to health outcomes. Although intake and status are correlated, they do not necessarily reflect the same. Intake is determined by the diet consumed, whereas status is affected by absorption, distribution, metabolism and excretion [33]. Nutrient status is a measure of the amount of nutrient available to the body and the link between intake and disease development. Nutrient status can thus provide information on the mechanism underlying a diet-disease association [34]. For example, vitamin D status is dependent on both intake of vitamin D and production of vitamin D in the skin under influence of sunlight [35]. The biological effect of vitamin D intake on health outcomes will be mediated by vitamin D status [36]. In short, intake and status provide complementary information. However, studies examining both dietary intake and nutrient status during pregnancy are limited, with studies reporting either only dietary intakes $[32,37$, 38] or nutrient status markers [39-41]. 
Figure 1.1: The DISH model representing the link between diet and health through determinants, intake and status [42].

\section{Gestational diabetes}

\section{Definition and prevalence}

Gestational diabetes mellitus (GDM) is one of the most common metabolic complications during pregnancy [43]. GDM is defined as carbohydrate intolerance or hyperglycaemia (high blood glucose concentration) with first onset or recognition during pregnancy [44]. In 2015, globally, one in eight births was affected by GDM according to the International Diabetes Federation estimates [45]. However, a lack of consensus regarding the appropriate diagnostic criteria, and the selective one-step or universal two-step screening (Box 1.1) have hampered accurate prevalence rates of GDM [46, 47]. Depending on the population studied and the diagnostic criteria used, GDM prevalence estimates range from $1-18 \%$ of all pregnancies $[46,48]$. Across Europe, including the Netherlands, prevalence of GDM is estimated to be around 2-5\% of all pregnancies [46], with prevalence going up to $25 \%$ in highrisk groups $[49,50]$. The risk of developing GDM is increased in women with the following risk factors: high BMI, age $>25$ years, non-Caucasian ethnicity, family history of diabetes, GDM in a previous pregnancy, chronic hypertension, large abdominal circumference, high fasting glycaemia in the first trimester of pregnancy, and the presence of polycystic ovary syndrome [51,52]. With the increasing burden of obesity among women of reproductive age, the prevalence of GDM is increasing [53]. In the USA, prevalence of GDM has increased from $0.3 \%$ in 1979-1980 to 5.8\% in 2008-2010 [54], affecting over 110,000 women every year [55]. Increasing rates of GDM have also been observed in Europe, Australia and Asia [56-60]. 
Box 1.1: Screening and diagnosis of gestational diabetes (GDM)

Screening for GDM is done at 24-28 weeks of gestation using either a universal two-step diagnosis strategy or a selective one-step diagnosis strategy.

Selective one-step diagnosis strategy: Pregnant women with one or more risk factors for GDM undergo a 100 grams or 75 grams oral glucose tolerance test (OGTT) after an overnight fast.

Universal two-step diagnosis strategy: All pregnant women undergo a non-fasting 50 grams glucose challenge test. After one hour a blood sample is drawn. Women with a one-hour glucose value $\geq 7.8$ $\mathrm{mmol} / \mathrm{L}$ undergo a 100 grams or 75 grams OGTT after an overnight fast.

A positive GDM diagnosis is given using one of the diagnostic criteria sets displayed below.

Commonly used diagnostic criteria for GDM after an OGTT

\begin{tabular}{llllll}
\hline & NDDG (1979) [61] & \multicolumn{2}{c}{ ADA (1997) [62] } & WHO (1999) [63] & IADPSG $^{\text {(2010) [64] }}$ \\
\cline { 2 - 6 } & 100-g OGTT & $100-\mathrm{g} \mathrm{OGTT}$ & 75-g OGTT & 75-g OGTTc & 75-g OGTTc \\
\hline Fasting glucose & $\geq 5.8 \mathrm{mmol} / \mathrm{L}$ & $\geq 5.3 \mathrm{mmol} / \mathrm{L}$ & $\geq 5.3 \mathrm{mmol} / \mathrm{L}$ & $\geq 6.1 \mathrm{mmol} / \mathrm{L}$ & $\geq 5.1 \mathrm{mmol} / \mathrm{L}$ \\
1 hour glucose & $\geq 10.5 \mathrm{mmol} / \mathrm{L}$ & $\geq 10.0 \mathrm{mmol} / \mathrm{L}$ & $\geq 10.0 \mathrm{mmol} / \mathrm{L}$ & - & $\geq 10.0 \mathrm{mmol} / \mathrm{L}$ \\
2 hour glucose & $\geq 9.1 \mathrm{mmol} / \mathrm{L}$ & $\geq 8.6 \mathrm{mmol} / \mathrm{L}$ & $\geq 8.6 \mathrm{mmol} / \mathrm{L}$ & $\geq 7.8 \mathrm{mmol} / \mathrm{L}$ & $\geq 8.5 \mathrm{mmol} / \mathrm{L}$ \\
3 hour glucose & $\geq 8.0 \mathrm{mmol} / \mathrm{L}$ & $\geq 7.8 \mathrm{mmol} / \mathrm{L}$ & - & - & - \\
\hline
\end{tabular}

NDDG, National Diabetes Data Group; ADA, American Diabetes Association; WHO, World Health Organization; IADPSG, International Association of Diabetes and Pregnancy Study Group; OGTT, oral glucose tolerance test

${ }^{1}$ After publication of IADPSG diagnostic criteria, ADA and WHO endorse the use of the IADPSG criteria.

a Glucose tolerance test with $100 \mathrm{~g}$ of oral glucose: two abnormal values at any time of the curve indicate GDM.

${ }^{b}$ Glucose tolerance test with $75 \mathrm{~g}$ of oral glucose: two abnormal values at any time of the curve indicate GDM.

Although diagnostic criteria for the 75-g OGTT are defined, the ADA recommends the use of the 100-g OGTT.

c Glucose tolerance test with $75 \mathrm{~g}$ of oral glucose: one or more abnormal values indicate GDM.

\section{Adverse health effects associated with GDM}

GDM has been related to significant short-term and long-term adverse health outcomes for both mothers and offspring [65-68]. In 2008 the results from the landmark Hyperglycaemia and Adverse Pregnancy Outcome (HAPO) study were published and showed that glucose levels during pregnancy are linearly related to risk of adverse pregnancy outcomes including birth weight $>90$ th percentile, primary Caesarean section, shoulder dystocia, neonatal hyperbilirubinemia and neonatal hypoglycaemia [69]. Women with a history of GDM are seven times more likely to develop type 2 diabetes mellitus (T2DM) than women without GDM and the onset of T2DM after GDM occurs already within five to ten years after the GDM pregnancy [70, 71]. Furthermore, women with a history of GDM have a $30-40 \%$ higher risk of cardiovascular events, for women who develop T2DM after GDM the risk of cardiovascular events is even three-fold higher compared to women without a history of GDM [72, 73]. 
Children born to mothers with GDM have an increased risk of developing overweight, the metabolic syndrome and T2DM during childhood, adolescence, and adulthood [74, 75]. Uncontrolled GDM leads to a hyperglycaemic intra-uterine environment, as glucose can cross the placenta and insulin cannot. This may lead to overstimulation of foetal beta cells, accelerating foetal growth and fat disposition and is also known as the Pedersen's hypothesis [76]. Furthermore, epigenetic changes in response to the hyperglycaemic intra-uterine environment are thought to be a likely mechanism by which the intrauterine environment affects health and disease of the offspring of GDM mothers [77, 78].

\section{Pathophysiology of GDM}

Over the course of normal pregnancy, insulin sensitivity decreases by $50-60 \%$ [79]. The decrease in insulin sensitivity during normal pregnancy is thought to be the result of increased maternal adiposity, production of insulin desensitizing hormones by the placenta, subclinical inflammation, and reduced adiponectin secretion [80]. The proposed key cellular mechanism is the post-receptor insulin-signalling cascade including the insulin receptor substrate 1 tyrosine phosphorylation [80, 81]. GDM results from an imbalance between decreased insulin sensitivity and the capacity of pancreatic beta cells to compensate by increasing insulin production. Most women who develop GDM have subclinical metabolic dysfunction, including impaired insulin response, already prior to conception [81].

\section{Treatment and prevention}

After GDM diagnosis, women receive treatment from medical care providers. The primary approach for GDM treatment is dietary advice in combination with self-monitoring of blood glucose levels [82]. Main aim of the treatment is to achieve optimal glycaemic control, i.e. regulation and maintenance of blood glucose levels within the normal range. Although there are no specific guidelines for diet in GDM, dietary advice mostly includes carbohydrate distribution and a reduction in rapidly digestible sugars. Seventy to $85 \%$ of the women with GDM achieve glycaemic control by adjustments to their diet [82]. Women who fail to maintain glycaemic control within 1-2 weeks generally receive additional insulin therapy.

Two large randomized controlled trials showed that treatment of GDM consisting of dietary counselling, self-monitoring of blood glucose and, if needed, insulin therapy is effective in reducing adverse pregnancy outcomes, including shoulder dystocia, bone fracture, nerve palsy, Caesarean delivery and foetal overgrowth $[83,84]$. Although treatment is effective in reducing adverse pregnancy outcomes, it is not yet clear whether risk of adverse pregnancy outcomes for treated GDM is similar to risk of women with normal glucose tolerance. 
Prevention of GDM is desirable as this might reduce long-term adverse health outcomes of mother and offspring and puts less burden on health care systems and costs than GDM treatment $[85,86]$. As $\mathrm{BMI}$ is one of the most important risk factors for development of GDM, prevention of GDM has focused on modifiable factors including diet, exercise and weight $[87,88]$. As diet plays a central role in GDM treatment and there is substantial evidence relating dietary factors to glucose homeostasis [89, 90], the potential role of diet in GDM prevention has increasingly received attention [91, 92].

\section{Diet and GDM prevention}

A few intervention studies have investigated a dietary intervention, or lifestyle intervention with a dietary component, to prevent GDM development $[93,94]$. Several have found a reduced incidence of GDM in the intervention group. One RCT included 50 Danish obese pregnant women, and the intervention group receiving 10 one-hour consultations with a dietician had significantly less deterioration in glucose homeostasis than the control group [95]. One non-randomized trial including 185 Finnish mothers at risk of GDM receiving diet and physical activity counselling, starting at the end of the first trimester, reported a 50\% reduction in GDM incidence compared with the control group [96]. The largest RCT in 400 Finnish women with at least one risk factor for GDM receiving intensive individual counselling on diet and physical activity showed a 39\% reduction in GDM prevalence compared to standard care [97]. However, others, including a large European multicentre trial in overweight and obese pregnant women, did not find a reduction in GDM prevalence after lifestyle or dietary counselling [98-101]. Differences in dietary intervention (e.g. focus on macronutrient composition, low glycaemic (GI) index, healthy dietary pattern), intensity of the intervention, start of the intervention (e.g. first trimester, second trimester) and study population (e.g. obese pregnant women, women who had GDM in a previous pregnancy) might account for differences in effect. Thus, no definite conclusion on the role of diet in GDM prevention can be drawn from these intervention studies.

Most evidence on the associations between diet and GDM comes from observational studies, and has been summarized by two recent reviews [91, 93]. These reviews give a comprehensive overview of studies investigating dietary factors and the development of GDM. A summary of their results is presented in Table 1.1. A wide range of dietary factors has been investigated, but results per dietary factor are limited to only a few studies and, together with the observational nature of the studies, the associations between individual dietary factors and GDM development remain inconclusive. Several observational studies have investigated dietary patterns in relation to GDM risk. Most studies observed a lower risk of GDM when adhering to a healthy dietary pattern, e.g. Mediterranean diet, prudent 
dietary pattern [102-111]. These healthy dietary patterns are often characterized by a high intake of fruit, vegetables, whole grain products and a low intake of animal and processed food products.

Only a few studies have investigated nutrient status of different micronutrients and the association with GDM development. A high folate status [112] and vitamin D deficiency [113] have been linked to a higher risk of GDM, whereas a lower risk of GDM was observed with higher levels of vitamin B12 $[112,114,115]$, vitamin C [116], zinc and selenium [117].

Current evidence from observational studies is dominated by findings from the Nurses' Health Study II, which may not be representative for other populations and incomplete adjustment or clustering of health behaviours may confound reported associations [91]. Furthermore, detailed dietary assessments and blood samples are lacking and GDM diagnosis relies on self-report of participants. Furthermore, dietary assessment is done either before pregnancy or once during pregnancy, missing potential changes in dietary intake occurring due to getting pregnant. The role of nutrient status in the preconception period as well as the influence of changes in dietary intake from preconception to GDM diagnosis remain to be investigated.

Table 1.1: Summary of associations between dietary intake and gestational diabetes mellitus reported in observational studies, stratified for study design. Adapted from Schoenaker et al. [91] and Donazar et al. [93].

\begin{tabular}{|c|c|c|c|c|c|}
\hline & \multicolumn{4}{|c|}{ Study design } & \multirow[b]{2}{*}{ References } \\
\hline & $\#$ & Prospective & $\begin{array}{l}\text { Cross- } \\
\text { sectional }\end{array}$ & Case-control & \\
\hline \multicolumn{6}{|c|}{ Energy and macronutrients } \\
\hline Total energy & 14 & $x x x x x$ & $\uparrow x x x$ & $x x x x x$ & [117-130] \\
\hline Protein & 11 & $x x x x$ & $\uparrow x x$ & $x x x x$ & $\begin{array}{l}{[118,120,121,123-125,128-} \\
132]\end{array}$ \\
\hline Carbohydrates & 12 & $x x x x \downarrow$ & $\uparrow \times \downarrow$ & $x x x \downarrow$ & $\begin{array}{l}{[118,120,121,123-125,127-} \\
130,132,133]\end{array}$ \\
\hline Dietary fibre & 10 & $x x \downarrow$ & $\uparrow x x x$ & $x \downarrow \downarrow$ & {$[119-122,124-127,130,133]$} \\
\hline Total fat & 13 & $\uparrow \uparrow \uparrow x x x$ & $\uparrow \uparrow x$ & $x x x \downarrow$ & $\begin{array}{l}{[118-121,123-125,127-130,132,} \\
134]\end{array}$ \\
\hline Saturated fat & 10 & $x x$ & $x x x$ & 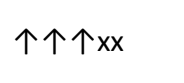 & $\begin{array}{l}{[120-122,124,126-128,130} \\
132,134]\end{array}$ \\
\hline Monounsaturated fat & 9 & $x x$ & $x x$ & $\uparrow x x x \downarrow$ & $\begin{array}{l}{[120,121,124,126-128,130,} \\
132,134]\end{array}$ \\
\hline Polyunsaturated fat & 9 & $x x$ & $\uparrow x$ & $\uparrow x \downarrow \downarrow \downarrow$ & $\begin{array}{l}{[120,121,124,126-128,130,} \\
132,134]\end{array}$ \\
\hline Omega-3 fatty acids & 4 & $\uparrow x$ & & $\uparrow$ & {$[126,127,134]$} \\
\hline Omega- 6 fatty acids & 3 & $x x$ & & & {$[127,134]$} \\
\hline Trans fat & 5 & $x x$ & $x x$ & $\uparrow$ & {$[121,124,127,130,134]$} \\
\hline PUFA:SAFA ratio & 5 & $x x$ & $x x$ & $\downarrow$ & {$[121,124,127,130,134]$} \\
\hline Cholesterol & 9 & $\uparrow \uparrow$ & $\uparrow x$ & $\uparrow x$ & {$[121,124,126,128,134]$} \\
\hline
\end{tabular}


Micronutrients

$[122,135,136]$

xx

$\begin{array}{ll}\text { Iron } & 3 \\ \text { Zinc } & 2 \\ \text { Selenium } & 1 \\ \text { Vitamin A } & 1 \\ \text { Vitamin E } & 3 \\ \text { Vitamin C } & 4 \\ \text { Magnesium } & 2\end{array}$

$[117,135]$

$x$

$\downarrow$

[117]

[117]

[117, 126, 137]

xx

$[116,117,126,138]$

$x \downarrow \downarrow$

$[128,133]$

\section{Dietary patterns}

Mediterranean diet

DASH diet

$3 \downarrow \downarrow$

$\downarrow$

Healthy Eating Index

Prudent

Western

Low-Carbohydrate Diet

[104, 105, 109]

$[104,109]$

$[109,110]$

$[102,103,107,110,111]$

$[102,103,107,108,111]$

[139]

Foods

\begin{tabular}{|c|c|c|c|}
\hline Fruits and vegetables & 1 & $x$ & \\
\hline Fruit & 3 & $x x$ & \\
\hline Fruit juice & 2 & $x x$ & \\
\hline Vegetables & 2 & $\mathrm{x}$ & \\
\hline Potato & 2 & $\uparrow \downarrow$ & \\
\hline Legumes & 2 & $x x$ & \\
\hline Dairy products & 3 & $x x$ & \\
\hline Cheese & 1 & $\uparrow$ & \\
\hline Red meat & 4 & 个个x & \\
\hline Processed meat & 4 & 个个x & \\
\hline Poultry & 1 & $x$ & \\
\hline Fish & 3 & $x x$ & $x$ \\
\hline Eggs & 3 & $x \uparrow$ & \\
\hline Nuts & 1 & $\downarrow$ & \\
\hline Whole grains & 2 & $x$ & \\
\hline Cereal & 1 & $\downarrow$ & \\
\hline Fried foods & 2 & 个个 & \\
\hline Sugar-sweetened cola & 1 & $\uparrow$ & \\
\hline $\begin{array}{l}\text { Sugar-sweetened } \\
\text { beverages (not cola) }\end{array}$ & 1 & $x$ & \\
\hline Diet cola & 1 & $x$ & \\
\hline $\begin{array}{l}\text { Other diet beverages (not } \\
\text { cola) }\end{array}$ & 1 & $x$ & \\
\hline Coffee & 1 & $x$ & \\
\hline Tea & 1 & $x$ & \\
\hline Olive oil & 1 & $\uparrow$ & \\
\hline
\end{tabular}

[123, 126, 141]

$[118,141]$

$[123,126]$

$[123,142]$

$[123,131]$

$[123,126,131]$

[123]

$[111,126,127,131]$

[111, 126, 127, 131]

[131]

$[123,131,143]$

$[126,131]$

[131]

$[126,127]$

[123]

$[119,144]$

PUFA, polyunsaturated fatty acids; SAFA, saturated fatty acids; DASH, dietary approaches to stop hypertension 个 Significant positive association between dietary intake and risk of gestational diabetes mellitus $(\mathrm{P}<0.05)$ $\downarrow$ Significant inverse association between dietary intake and risk of gestational diabetes mellitus $(P<0.05)$ $x$ No statistically significant association between dietary intake and gestational diabetes mellitus 


\section{Dietary assessment issues}

To study dietary intakes during pregnancy and the association between diet and GDM development dietary assessment is essential. However, there are some obstacles in dietary assessment. The concept of diet is complex as dietary intake can be expressed as intake of single nutrients, food items, food groups or dietary patterns. Foods and nutrients are consumed in combinations, which can induce interactions and synergies between dietary components. Therefore, dietary pattern analysis is assumed a more appropriate approach for investigating diet-disease associations than focusing on a single food or nutrient [147]. Furthermore, a dietary pattern represents a single summary measure of one's diet. Dietary guidelines represent the current state of scientific knowledge and are designed to prevent nutrient deficiencies and chronic diseases. A dietary pattern index score assessing adherence to dietary guidelines can be used as a measure of diet quality [148]. However, dietary guidelines may differ between countries and index scores need to be properly designed and evaluated before they can be used in research.

A second issue in dietary assessment is the measurement error present in dietary intake estimates. Food-frequency questionnaires (FFQ) and 24-hour recalls (24hR) are commonly used dietary assessment methods. These methods both have their strengths and limitations [34]. The FFQ is, for example, relatively cheap and easy to administer, but relies on memory and can lead to social desirable answers, while a limited set of aggregated food items leads to loss of precision, and portion sizes are difficult to assess accurately. The $24 \mathrm{hR}$ assesses all foods consumed on a single day, but also relies on memory and can lead to social desirable answers. Furthermore, multiple recalls are necessary to capture individual habitual intake. Altogether, dietary intake estimates assessed with the FFQ or $24 \mathrm{hR}$ are known to be biased due to random and systematic measurement error, which can lead to biased associations and reduced power $[149,150]$. Statistical methods can partly correct the bias in diethealth associations introduced by measurement errors, but require intake estimates from a second (superior) assessment method, i.e. a reference method.

\section{Aim and outline of the thesis}

This thesis aims to provide more insight into dietary intake and nutrient status before and during pregnancy and into the association of dietary intake and nutrient status with development of GDM. To achieve this overall aim, one objective and four research questions were defined: 
- Develop an index to assess adherence to the Dutch dietary guidelines of 2015

- Does combining dietary data of a FFQ with dietary data of $24 \mathrm{hR}$ reduce bias in diet-disease associations?

- Are women with GDM who receive treatment at increased risk of common adverse pregnancy outcomes?

- What is the association between dietary intake, nutrient status and development of GDM?

- How do dietary intake, supplement use and nutrient status change during pregnancy?

The first part of the thesis focusses on two methodological issues of nutrition research. In chapter $\mathbf{2}$, the development and evaluation of the Dutch Healthy Diet index 2015 (DHD15 index) score assessing adherence to the Dutch dietary guidelines of 2015 is described. Chapter 3 describes the impact of combining $24 \mathrm{hR}$ and FFQ estimates using (enhanced) regression calibration on diet-disease associations. In both chapters data from the NQplus study is used, see box $\mathbf{1 . 2}$ for details of the study. The next chapter, chapter 4, focusses on adverse pregnancy outcomes related to GDM, by comparing adverse pregnancy outcomes of women who develop GDM and receive treatment to pregnant women with untreated borderline GDM and pregnant women with normal glucose tolerance. For this chapter, data from medical records (GLIMP study) is used, see box 1.2. In the next chapters, the association of pre-pregnancy dietary intake and the development of GDM is examined. Chapter $\mathbf{5}$ focusses on the association between pre-pregnancy carbohydrate intake and development of GDM and in chapter 6, the association between pre-pregnancy micronutrient intake and development of GDM is described. Data from the prospective cohort study Australian Longitudinal Study on Women's Health (ALSWH) is used in chapters 5 and 6 , see box 1.2. Dietary intake and supplement use might change over the course of the pregnancy, and consequently influence nutrient status. This is described in chapter $\mathbf{7}$, as well as the association of diet quality, micronutrient intake and status with glucose tolerance markers measured in the preconception period, at the end of the first trimester and the end of the second trimester. In the final part of this thesis, focus lies on dietary intakes in the preconception period. In chapter 8, dietary intake in the preconception period is examined in more detail with a description of supplement use and dietary sources of folate, vitamin $D$, and $n-3$ fatty acids and the correlation between supplemental and dietary intake with blood levels is examined. Chapter 7 and 8 use data from the small-scale GLIMP2 study, see box 1.2. In the final chapter of this thesis, chapter 9, the main findings of the studies are summarized and discussed. This general discussion puts the findings into perspective and gives implications for practice and further suggestions for future research. 
Box 1.2: Characteristics of the studies used in this thesis.

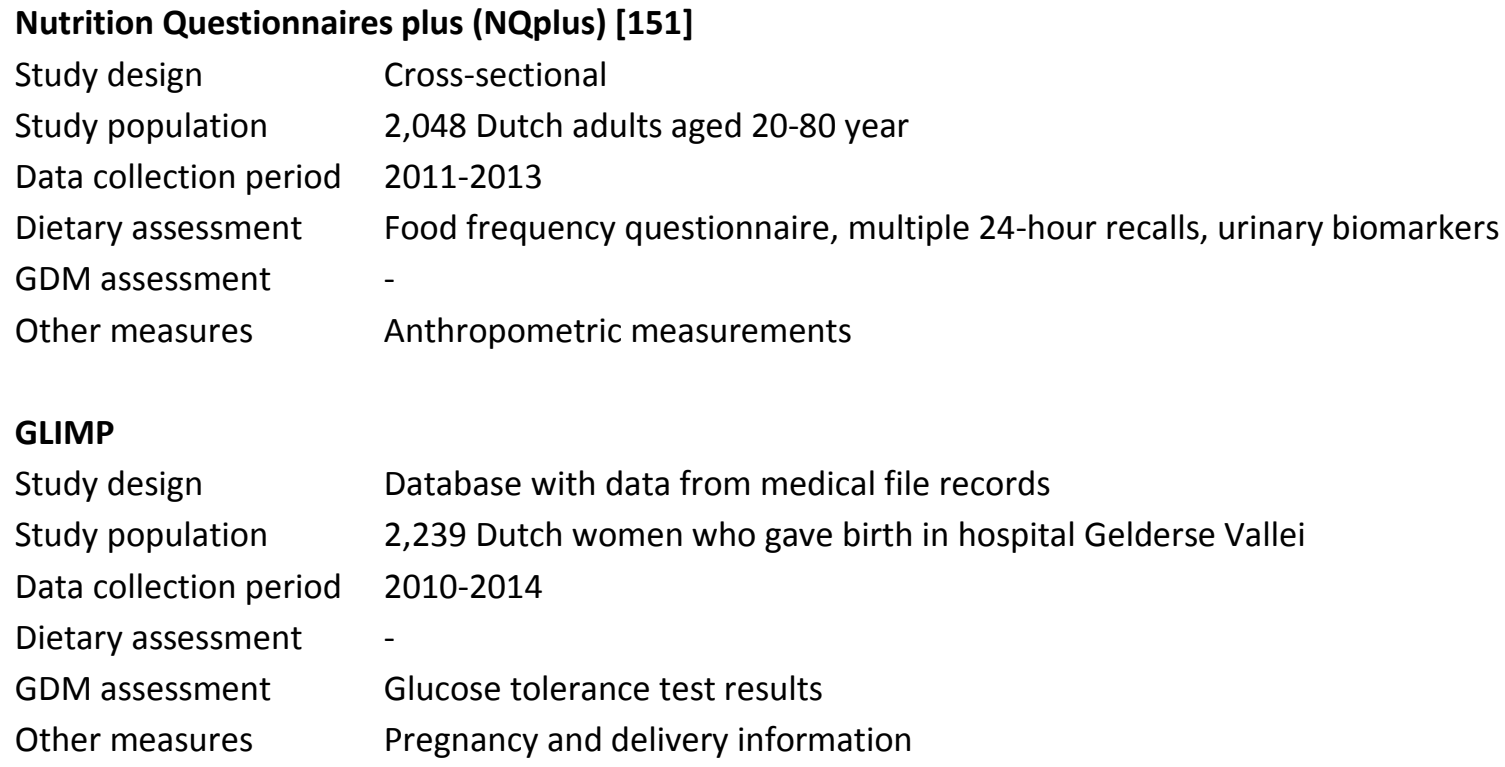

\section{GLIMP2}

Study design Longitudinal prospective study, measurements at preconception, 12 weeks pregnant, 24 weeks pregnant, and 6-12 weeks after delivery

Study population $\quad 115$ Dutch women aged 18-40 with either a wish to get pregnant within one year or those less than 24 weeks pregnant

Data collection period

Dietary assessment

2015-2017

markers

GDM assessment Glucose tolerance test results

Other measures Anthropometrics, supplement use 


\section{References}

1. Abu-Saad, K. and D. Fraser, Maternal nutrition and birth outcomes. Epidemiol. Rev., 2010. 32: p. 5-25.

2. Berti, C., H.K. Biesalski, R. Gartner, et al., Micronutrients in pregnancy: current knowledge and unresolved questions. Clin. Nutr., 2011. 30(6): p. 689-701.

3. Roseboom, T.J., J.H. van der Meulen, A.C. Ravelli, et al., Effects of prenatal exposure to the Dutch famine on adult disease in later life: an overview. Mol. Cell. Endocrinol., 2001. 185(1-2): p. 93-8.

4. Kaiser, L., L.H. Allen, and American Dietetic Association, Position of the American Dietetic Association: nutrition and lifestyle for a healthy pregnancy outcome. J. Am. Diet. Assoc., 2008. 108(3): p. 553-61.

5. Roseboom, T.J., R.C. Painter, A.F. van Abeelen, et al., Hungry in the womb: what are the consequences? Lessons from the Dutch famine. Maturitas, 2011. 70(2): p. 141-5.

6. Barker, D.J.P., K.M. Godfrey, P.D. Gluckman, et al., Fetal nutrition and cardiovascular disease in adult life. The Lancet, 1993. 341(8850): p. 938-941.

7. Jehn, M. and A. Brewis, Paradoxical malnutrition in mother-child pairs: untangling the phenomenon of over-and under-nutrition in underdeveloped economies. Econ. Hum. Biol., 2009. 7(1): p. 28-35.

8. Neri, C. and A.G. Edlow, Effects of Maternal Obesity on Fetal Programming: Molecular Approaches. Cold Spring Harb. Perspect. Med., 2015. 6(2): p. a026591.

9. Health Council of the Netherlands, Towards an adequate intake of vitamins and minerals. Publication no. 2009/06E. 2009, Health council of the Netherlands: The Hague.

10. Institute of Medicine, Nutrition During Pregnancy. 1990, National Academies Press: Washington DC.

11. National Health and Medical Research Council, Nutrient Reference Values for Australia and New Zealand. 2005, NHMRC: Canberra.

12. Scientific Advisory Committee on Nutrition, Dietary Reference Values for Energy. 2011, TSO: London.

13. European Food Safety Authority, Dietary Reference Values for nutrients: Summary report. 2017, EFSA supporting publication 2017:e15121.

14. Blumfield, M.L., A.J. Hure, L. Macdonald-Wicks, et al., Systematic review and meta-analysis of energy and macronutrient intakes during pregnancy in developed countries. Nutr. Rev., 2012. 70(6): p. 322-36.

15. Goletzke, J., A.E. Buyken, J.C.Y. Louie, et al., Dietary micronutrient intake during pregnancy is a function of carbohydrate quality. Am. J. Clin. Nutr., 2015. 102(3): p. 626-632.

16. Cogswell, M.E., G.S. Perry, L.A. Schieve, et al., Obesity in women of childbearing age: risks, prevention, and treatment. Prim. Care Update Ob Gyns, 2001. 8(3): p. 89-105.

17. Helms, E., C.C. Coulson, and S.L. Galvin, Trends in weight gain during pregnancy: a population study across 16 years in North Carolina. Am. J. Obstet. Gynecol., 2006. 194(5): p. e32-4.

18. Schieve, L.A., M.E. Cogswell, and K.S. Scanlon, Trends in pregnancy weight gain within and outside ranges recommended by the Institute of Medicine in a WIC population. Matern Child Health J, 1998. 2(2): p. 111-6.

19. Lof, M., Physical activity pattern and activity energy expenditure in healthy pregnant and non-pregnant Swedish women. Eur. J. Clin. Nutr., 2011. 65(12): p. 1295-301.

20. Lumley, J., L. Watson, M. Watson, et al., Periconceptional supplementation with folate and/or multivitamins for preventing neural tube defects. The Cochrane database of systematic reviews, 2001(3): p. CD001056.

21. Cusick, S.E. and M.K. Georgieff, Nutrient supplementation and neurodevelopment: timing is the key. Arch. Pediatr. Adolesc. Med., 2012. 166(5): p. 481-2.

22. Villamor, E., S.L. Rifas-Shiman, M.W. Gillman, et al., Maternal intake of methyl-donor nutrients and child cognition at 3 years of age. Paediatr. Perinat. Epidemiol., 2012. 26(4): p. 328-35.

23. Hermoso, M., V. Vucic, C. Vollhardt, et al., The effect of iron on cognitive development and function in infants, children and adolescents: a systematic review. Ann. Nutr. Metab., 2011. 59(2-4): p. 154-65.

24. Szajewska, H., M. Ruszczynski, and A. Chmielewska, Effects of iron supplementation in nonanemic pregnant women, infants, and young children on the mental performance and psychomotor development of children: a systematic review of randomized controlled trials. Am. J. Clin. Nutr., 2010. 91(6): p. 1684-90.

25. Uriu-Adams, J.Y. and C.L. Keen, Zinc and reproduction: effects of zinc deficiency on prenatal and early postnatal development. Birth Defects Res. B Dev. Reprod. Toxicol., 2010. 89(4): p. 313-25.

26. Williamson, C.S., Nutrition in pregnancy. Nutr. Bull., 2006. 31(1): p. 28-59.

27. Aghajafari, F., T. Nagulesapillai, P.E. Ronksley, et al., Association between maternal serum 25-hydroxyvitamin D level and pregnancy and neonatal outcomes: systematic review and meta-analysis of observational studies. BMJ, 2013. 346: p. f1169.

28. Weinert, L.S. and S.P. Silveiro, Maternal-fetal impact of vitamin D deficiency: a critical review. Matern Child Health J, 2015. 19(1): p. 94-101.

29. Blumfield, M.L., A.J. Hure, L. Macdonald-Wicks, et al., A systematic review and meta-analysis of micronutrient intakes during pregnancy in developed countries. Nutr. Rev., 2013. 71(2): p. 118-32.

30. World Health Organization, WHO recommendations on antenatal care for a positive pregnancy experience. 2016, World Health Organization: Geneva.

31. Health Council of the Netherlands, Towards an adequate intake of vitamin D. Publication no. 2008/15E, 2008: p. The Hague: Health Council of the Netherlands. 
32. Dubois, L., M. Diasparra, B. Bedard, et al., Adequacy of nutritional intake from food and supplements in a cohort of pregnant women in Quebec, Canada: the 3D Cohort Study (Design, Develop, Discover). Am. J. Clin. Nutr., 2017. 106(2): p. 541-548.

33. Potischman, N., Biologic and methodologic issues for nutritional biomarkers. J. Nutr., 2003. 133 Suppl 3: p. 875S$880 \mathrm{~S}$.

34. Willett WC, Nutritional Epidemiology. 3rd ed. 2013, New York: Oxford University Press.

35. Brouwer-Brolsma, E.M., A.M. Vaes, N.L. van der Zwaluw, et al., Relative importance of summer sun exposure, vitamin D intake, and genes to vitamin D status in Dutch older adults: The B-PROOF study. The Journal of steroid biochemistry and molecular biology, 2016. 164: p. 168-176.

36. Holick, M.F. and T.C. Chen, Vitamin D deficiency: a worldwide problem with health consequences. Am. J. Clin. Nutr., 2008. 87(4): p. 1080S-6S.

37. Crozier, S.R., S.M. Robinson, K.M. Godfrey, et al., Women's dietary patterns change little from before to during pregnancy. J. Nutr., 2009. 139(10): p. 1956-63.

38. Gresham, E., C.E. Collins, G.D. Mishra, et al., Diet quality before or during pregnancy and the relationship with pregnancy and birth outcomes: the Australian Longitudinal Study on Women's Health. Public Health Nutr., 2016. 19(16): p. 2975-2983.

39. Cikot, R.J., R.P. Steegers-Theunissen, C.M. Thomas, et al., Longitudinal vitamin and homocysteine levels in normal pregnancy. Br. J. Nutr., 2001. 85(1): p. 49-58.

40. Milman, N., T. Bergholt, K.E. Byg, et al., Reference intervals for haematological variables during normal pregnancy and postpartum in 434 healthy Danish women. Eur. J. Haematol., 2007. 79(1): p. 39-46.

41. Zhao, Y., W. Miao, C. Li, et al., Dynamic changes in serum 25-hydroxyvitamin D during pregnancy and lack of effect on thyroid parameters. PLoS One, 2014. 9(3): p. e90161.

42. Brown, K.A., L. Timotijevic, M. Geurts, et al., Concepts and procedures for mapping food and health research infrastructure: New insights from the EuroDISH project. Trends Food Sci Tech, 2017. 63: p. 113-131.

43. American Diabetes Association, Diagnosis and classification of diabetes mellitus. Diabetes Care, 2014. 37 Suppl 1: p. S81-90.

44. World Health Organization, Diagnostic criteria and classification of hyperglycaemia first detected in pregnancy. 2013, Geneva, Switzerland: World Health Organization.

45. International Diabetes Federation, IDF diabetes at/as. 2015: Seventh Edition.

46. Buckley, B.S., J. Harreiter, P. Damm, et al., Gestational diabetes mellitus in Europe: prevalence, current screening practice and barriers to screening. A review. Diabet. Med., 2012. 29(7): p. 844-54.

47. Koning, S.H., K. Hoogenberg, H.L. Lutgers, et al., Gestational Diabetes Mellitus:current knowledge and unmet needs. J. Diabetes, 2016. 8(6): p. 770-781.

48. Bardenheier, B.H., G. Imperatore, S.M. Gilboa, et al., Trends in Gestational Diabetes Among Hospital Deliveries in 19 U.S. States, 2000-2010. Am. J. Prev. Med., 2015. 49(1): p. 12-9.

49. Arendz, I.J., P.H. Oomen, A. Wolthuis, et al., [Prevalence of gestational diabetes in high-risk pregnancies: screened using an oral glucose tolerance test]. Ned. Tijdschr. Geneeskd., 2013. 157(18): p. A5409.

50. MacNeill, S., L. Dodds, D.C. Hamilton, et al., Rates and risk factors for recurrence of gestational diabetes. Diabetes Care, 2001. 24(4): p. 659-62.

51. Chen, P., S. Wang, J. Ji, et al., Risk factors and management of gestational diabetes. Cell Biochem. Biophys., 2015. 71(2): p. 689-94.

52. Popova, P.V., E.N. Grineva, A.S. Gerasimov, et al., The new combination of risk factors determining a high risk of gestational diabetes mellitus. Minerva Endocrinol., 2015. 40(4): p. 239-47.

53. Dabelea, D., J.K. Snell-Bergeon, C.L. Hartsfield, et al., Increasing prevalence of gestational diabetes mellitus (GDM) over time and by birth cohort: Kaiser Permanente of Colorado GDM Screening Program. Diabetes Care, 2005. 28(3): p. $579-84$.

54. Lavery, J.A., A.M. Friedman, K.M. Keyes, et al., Gestational diabetes in the United States: temporal changes in prevalence rates between 1979 and 2010. BJOG, 2017. 124(5): p. 804-813.

55. Getahun, D., C. Nath, C.V. Ananth, et al., Gestational diabetes in the United States: temporal trends 1989 through 2004. Am. J. Obstet. Gynecol., 2008. 198(5): p. 525 e1-5.

56. Chamberlain, C., E. Banks, G. Joshy, et al., Prevalence of gestational diabetes mellitus among Indigenous women and comparison with non-Indigenous Australian women: 1990-2009. Aust. N. Z. J. Obstet. Gynaecol., 2014. 54(5): p. 433-40.

57. Helseth, R., O. Salvesen, S.N. Stafne, et al., Gestational diabetes mellitus among Nordic Caucasian women: prevalence and risk factors according to WHO and simplified IADPSG criteria. Scand. J. Clin. Lab. Invest., 2014. 74(7): p. 620-8.

58. Ignell, C., R. Claesson, E. Anderberg, et al., Trends in the prevalence of gestational diabetes mellitus in southern Sweden, 2003-2012. Acta Obstet. Gynecol. Scand., 2014. 93(4): p. 420-4.

59. Ovesen, P.G., D.M. Jensen, P. Damm, et al., Maternal and neonatal outcomes in pregnancies complicated by gestational diabetes. a nation-wide study. J. Matern. Fetal Neonatal Med., 2015. 28(14): p. 1720-4.

60. Zhang, F., L. Dong, C.P. Zhang, et al., Increasing prevalence of gestational diabetes mellitus in Chinese women from 1999 to 2008. Diabet. Med., 2011. 28(6): p. 652-7. 
61. National Diabetes Data Group, Classification and diagnosis of diabetes mellitus and other categories of glucose intolerance. Diabetes, 1979. 28(12): p. 1039-57.

62. American Diabetes Association, Report of the Expert Committee on the Diagnosis and Classification of Diabetes Mellitus. Diabetes Care, 1997. 20(7): p. 1183-97.

63. World Health Organization, Definition, diagnosis and classification of diabetes mellitus and its complications. 1999, World Health Organization: Geneva, Switzerland.

64. International Association of Diabetes Pregnancy Study Groups Consensus Panel, International association of diabetes and pregnancy study groups recommendations on the diagnosis and classification of hyperglycemia in pregnancy. Diabetes Care, 2010. 33(3): p. 676-82.

65. Boney, C.M., A. Verma, R. Tucker, et al., Metabolic syndrome in childhood: association with birth weight, maternal obesity, and gestational diabetes mellitus. Pediatrics, 2005. 115(3): p. e290-6.

66. Burguet, A., Long-term outcome in children of mothers with gestational diabetes. Diabetes Metab., 2010. 36(6 Pt 2): p. 682-94.

67. Cho, N.H., B.L. Silverman, T.A. Rizzo, et al., Correlations between the intrauterine metabolic environment and blood pressure in adolescent offspring of diabetic mothers. J. Pediatr., 2000. 136(5): p. 587-92.

68. Dabelea, D., R.L. Hanson, R.S. Lindsay, et al., Intrauterine exposure to diabetes conveys risks for type 2 diabetes and obesity: a study of discordant sibships. Diabetes, 2000. 49(12): p. 2208-11.

69. Hapo Study Cooperative Research Group, B.E. Metzger, L.P. Lowe, et al., Hyperglycemia and adverse pregnancy outcomes. N. Engl. J. Med., 2008. 358(19): p. 1991-2002.

70. Kim, C., K.M. Newton, and R.H. Knopp, Gestational diabetes and the incidence of type 2 diabetes: a systematic review. Diabetes Care, 2002. 25(10): p. 1862-8.

71. Bellamy, L., J.P. Casas, A.D. Hingorani, et al., Type 2 diabetes mellitus after gestational diabetes: a systematic review and meta-analysis. Lancet, 2009. 373(9677): p. 1773-9.

72. Brewster, S., B. Zinman, R. Retnakaran, et al., Cardiometabolic consequences of gestational dysglycemia. J. Am. Coll. Cardiol., 2013. 62(8): p. 677-84.

73. Retnakaran, R. and B.R. Shah, Role of Type 2 Diabetes in Determining Retinal, Renal, and Cardiovascular Outcomes in Women With Previous Gestational Diabetes Mellitus. Diabetes Care, 2017. 40(1): p. 101-108.

74. Clausen, T.D., E.R. Mathiesen, T. Hansen, et al., High prevalence of type 2 diabetes and pre-diabetes in adult offspring of women with gestational diabetes mellitus or type 1 diabetes: the role of intrauterine hyperglycemia. Diabetes Care, 2008. 31(2): p. 340-6.

75. Vaarasmaki, M., A. Pouta, P. Elliot, et al., Adolescent manifestations of metabolic syndrome among children born to women with gestational diabetes in a general-population birth cohort. Am. J. Epidemiol., 2009. 169(10): p. 120915.

76. Pedersen, J., Weight and length at birth of infants of diabetic mothers. Acta Endocrinol. (Copenh.), 1954. 16(4): p. 330-42.

77. Lehnen, H., U. Zechner, and T. Haaf, Epigenetics of gestational diabetes mellitus and offspring health: the time for action is in early stages of life. Mol. Hum. Reprod., 2013. 19(7): p. 415-22.

78. Mitanchez, D., C. Yzydorczyk, B. Siddeek, et al., The offspring of the diabetic mother - Short-and long-term implications. Best Pract. Res. Clin. Obstet. Gynaecol., 2014.

79. Catalano, P.M., L. Huston, S.B. Amini, et al., Longitudinal changes in glucose metabolism during pregnancy in obese women with normal glucose tolerance and gestational diabetes mellitus. Am. J. Obstet. Gynecol., 1999. 180(4): p. 903-16.

80. Barbour, L.A., C.E. McCurdy, T.L. Hernandez, et al., Cellular mechanisms for insulin resistance in normal pregnancy and gestational diabetes. Diabetes Care, 2007. 30 Suppl 2: p. S112-9.

81. Catalano, P.M., Trying to understand gestational diabetes. Diabet. Med., 2014. 31(3): p. 273-81.

82. American Diabetes Association, Gestational diabetes mellitus. Diabetes Care, 2004. 27 Suppl 1: p. S88-90.

83. Crowther, C.A., J.E. Hiller, J.R. Moss, et al., Effect of treatment of gestational diabetes mellitus on pregnancy outcomes. N. Engl. J. Med., 2005. 352(24): p. 2477-86.

84. Landon, M.B., C.Y. Spong, E. Thom, et al., A multicenter, randomized trial of treatment for mild gestational diabetes. N. Engl. J. Med., 2009. 361(14): p. 1339-48.

85. Halperin, I.J. and D.S. Feig, The role of lifestyle interventions in the prevention of gestational diabetes. Curr. Diab. Rep., 2014. 14(1): p. 452.

86. Veeraswamy, S., B. Vijayam, V.K. Gupta, et al., Gestational diabetes: the public health relevance and approach. Diabetes Res. Clin. Pract., 2012. 97(3): p. 350-8.

87. Farrar, D., L. Duley, N. Medley, et al., Different strategies for diagnosing gestational diabetes to improve maternal and infant health. The Cochrane database of systematic reviews, 2015. 1: p. CD007122.

88. Farrar, D., M. Simmonds, M. Bryant, et al., Treatments for gestational diabetes: a systematic review and metaanalysis. BMJ open, 2017. 7(6): p. e015557.

89. Heer, M. and S. Egert, Nutrients other than carbohydrates: their effects on glucose homeostasis in humans. Diabetes Metab. Res. Rev., 2015. 31(1): p. 14-35.

90. Russell, W.R., A. Baka, I. Bjorck, et al., Impact of Diet Composition on Blood Glucose Regulation. Crit. Rev. Food Sci. Nutr., 2016. 56(4): p. 541-90. 
91. Schoenaker, D.A., G.D. Mishra, L.K. Callaway, et al., The Role of Energy, Nutrients, Foods, and Dietary Patterns in the Development of Gestational Diabetes Mellitus: A Systematic Review of Observational Studies. Diabetes Care, 2016. 39(1): p. 16-23.

92. Zhang, C. and Y. Ning, Effect of dietary and lifestyle factors on the risk of gestational diabetes: review of epidemiologic evidence. Am. J. Clin. Nutr., 2011. 94(6 Suppl): p. 1975S-1979S.

93. Donazar-Ezcurra, M., C. Lopez-del Burgo, and M. Bes-Rastrollo, Primary prevention of gestational diabetes mellitus through nutritional factors: a systematic review. BMC Pregnancy Childbirth, 2017. 17.

94. Rogozinska, E., M. Chamillard, G.A. Hitman, et al., Nutritional manipulation for the primary prevention of gestational diabetes mellitus: a meta-analysis of randomised studies. PLoS One, 2015. 10(2): p. e0115526.

95. Wolff, S., J. Legarth, K. Vangsgaard, et al., A randomized trial of the effects of dietary counseling on gestational weight gain and glucose metabolism in obese pregnant women. Int. J. Obes. (Lond.), 2008. 32(3): p. 495-501.

96. Mustila, T., J. Raitanen, P. Keskinen, et al., Pragmatic controlled trial to prevent childhood obesity in maternity and child health care clinics: pregnancy and infant weight outcomes (the VACOPP Study). BMC Pediatr., 2013. 13: p. 80.

97. Koivusalo, S.B., K. Rono, M.M. Klemetti, et al., Gestational Diabetes Mellitus Can Be Prevented by Lifestyle Intervention: The Finnish Gestational Diabetes Prevention Study (RADIEL): A Randomized Controlled Trial. Diabetes Care, 2016. 39(1): p. 24-30.

98. Asbee, S.M., T.R. Jenkins, J.R. Butler, et al., Preventing excessive weight gain during pregnancy through dietary and lifestyle counseling: a randomized controlled trial. Obstet. Gynecol., 2009. 113(2 Pt 1): p. 305-12.

99. Markovic, T.P., R. Muirhead, S. Overs, et al., Randomized Controlled Trial Investigating the Effects of a Low-Glycemic Index Diet on Pregnancy Outcomes in Women at High Risk of Gestational Diabetes Mellitus: The GI Baby 3 Study. Diabetes Care, 2015.

100. Poston, L., R. Bell, H. Croker, et al., Effect of a behavioural intervention in obese pregnant women (the UPBEAT study): a multicentre, randomised controlled trial. The lancet. Diabetes \& endocrinology, 2015. 3(10): p. 767-77.

101. Simmons, D., R. Devlieger, A. van Assche, et al., Effect of Physical Activity and/or Healthy Eating on GDM Risk: The DALI Lifestyle Study. J. Clin. Endocrinol. Metab., 2017. 102(3): p. 903-913.

102. Donazar-Ezcurra, M., C. Lopez-Del Burgo, M.A. Martinez-Gonzalez, et al., Pre-pregnancy adherences to empirically derived dietary patterns and gestational diabetes risk in a Mediterranean cohort: the Seguimiento Universidad de Navarra (SUN) project. Br. J. Nutr., 2017. 118(9): p. 715-721.

103. He, J.R., M.Y. Yuan, N.N. Chen, et al., Maternal dietary patterns and gestational diabetes mellitus: a large prospective cohort study in China. Br. J. Nutr., 2015. 113(8): p. 1292-300.

104. Izadi, V., H. Tehrani, F. Haghighatdoost, et al., Adherence to the DASH and Mediterranean diets is associated with decreased risk for gestational diabetes mellitus. Nutrition, 2016. 32(10): p. 1092-6.

105. Karamanos, B., A. Thanopoulou, E. Anastasiou, et al., Relation of the Mediterranean diet with the incidence of gestational diabetes. Eur. J. Clin. Nutr., 2014. 68(1): p. 8-13.

106. Meinila, J., A. Valkama, S.B. Koivusalo, et al., Association between diet quality measured by the Healthy Food Intake Index and later risk of gestational diabetes-a secondary analysis of the RADIEL trial. Eur. J. Clin. Nutr., 2017. 71(4): p. 555-557.

107. Schoenaker, D.A., S.S. Soedamah-Muthu, L.K. Callaway, et al., Pre-pregnancy dietary patterns and risk of gestational diabetes mellitus: results from an Australian population-based prospective cohort study. Diabetologia, 2015. 58(12): p. 2726-35.

108. Shin, D., K.W. Lee, and W.O. Song, Dietary Patterns during Pregnancy Are Associated with Risk of Gestational Diabetes Mellitus. Nutrients, 2015. 7(11): p. 9369-82.

109. Tobias, D.K., C. Zhang, J. Chavarro, et al., Prepregnancy adherence to dietary patterns and lower risk of gestational diabetes mellitus. Am. J. Clin. Nutr., 2012. 96(2): p. 289-95.

110. Tryggvadottir, E.A., H. Medek, B.E. Birgisdottir, et al., Association between healthy maternal dietary pattern and risk for gestational diabetes mellitus. Eur. J. Clin. Nutr., 2016. 70(2): p. 237-42.

111. Zhang, C., M.B. Schulze, C.G. Solomon, et al., A prospective study of dietary patterns, meat intake and the risk of gestational diabetes mellitus. Diabetologia, 2006. 49(11): p. 2604-13.

112. Lai, J.S., W.W. Pang, S. Cai, et al., High folate and low vitamin B12 status during pregnancy is associated with gestational diabetes mellitus. Clin. Nutr., 2017.

113. Zhang, M.X., G.T. Pan, J.F. Guo, et al., Vitamin D Deficiency Increases the Risk of Gestational Diabetes Mellitus: A Meta-Analysis of Observational Studies. Nutrients, 2015. 7(10): p. 8366-75.

114. Krishnaveni, G.V., J.C. Hill, S.R. Veena, et al., Low plasma vitamin B12 in pregnancy is associated with gestational 'diabesity' and later diabetes. Diabetologia, 2009. 52(11): p. 2350-8.

115. Sukumar, N., H. Venkataraman, S. Wilson, et al., Vitamin B12 Status among Pregnant Women in the UK and Its Association with Obesity and Gestational Diabetes. Nutrients, 2016. 8(12).

116. Zhang, C., M.A. Williams, T.K. Sorensen, et al., Maternal plasma ascorbic Acid (vitamin C) and risk of gestational diabetes mellitus. Epidemiology, 2004. 15(5): p. 597-604.

117. Bo, S., A. Lezo, G. Menato, et al., Gestational hyperglycemia, zinc, selenium, and antioxidant vitamins. Nutrition, 2005. 21(2): p. 186-91.

118. Belzer, L.M., J.C. Smulian, S.E. Lu, et al., Food cravings and intake of sweet foods in healthy pregnancy and mild gestational diabetes mellitus. A prospective study. Appetite, 2010. 55(3): p. 609-15. 
119. Bennett, W.L., S.H. Liu, H.C. Yeh, et al., Changes in weight and health behaviors after pregnancies complicated by gestational diabetes mellitus: the CARDIA study. Obesity (Silver Spring), 2013. 21(6): p. 1269-75.

120. Bo, S., G. Menato, A. Lezo, et al., Dietary fat and gestational hyperglycaemia. Diabetologia, 2001. 44(8): p. 972-8.

121. Gonzalez-Clemente, J.M., O. Carro, I. Gallach, et al., Increased cholesterol intake in women with gestational diabetes mellitus. Diabetes Metab., 2007. 33(1): p. 25-9.

122. Helin, A., T.I. Kinnunen, J. Raitanen, et al., Iron intake, haemoglobin and risk of gestational diabetes: a prospective cohort study. BMJ open, 2012. 2(5).

123. Karamanos, B., A. Thanopoulou, E. Anastasiou, et al., Relation of the Mediterranean diet with the incidence of gestational diabetes. Eur. J. Clin. Nutr., 2013.

124. Ley, S.H., A.J. Hanley, R. Retnakaran, et al., Effect of macronutrient intake during the second trimester on glucose metabolism later in pregnancy. Am. J. Clin. Nutr., 2011. 94(5): p. 1232-40.

125. Park, S., M.Y. Kim, S.H. Baik, et al., Gestational diabetes is associated with high energy and saturated fat intakes and with low plasma visfatin and adiponectin levels independent of prepregnancy BMI. Eur. J. Clin. Nutr., 2013. 67(2): p. 196-201.

126. Qiu, C., I.O. Frederick, C. Zhang, et al., Risk of gestational diabetes mellitus in relation to maternal egg and cholesterol intake. Am. J. Epidemiol., 2011. 173(6): p. 649-58.

127. Radesky, J.S., E. Oken, S.L. Rifas-Shiman, et al., Diet during early pregnancy and development of gestational diabetes. Paediatr. Perinat. Epidemiol., 2008. 22(1): p. 47-59.

128. Rezvan, N., M.J. Hosseinzadeh-Attar, F. Masoudkabir, et al., Serum visfatin concentrations in gestational diabetes mellitus and normal pregnancy. Arch. Gynecol. Obstet., 2012. 285(5): p. 1257-62.

129. Saldana, T.M., A.M. Siega-Riz, and L.S. Adair, Effect of macronutrient intake on the development of glucose intolerance during pregnancy. Am. J. Clin. Nutr., 2004. 79(3): p. 479-86.

130. Wang, Y., L.H. Storlien, A.B. Jenkins, et al., Dietary variables and glucose tolerance in pregnancy. Diabetes Care, 2000. 23(4): p. 460-4.

131. Bao, W., K. Bowers, D.K. Tobias, et al., Prepregnancy dietary protein intake, major dietary protein sources, and the risk of gestational diabetes mellitus: a prospective cohort study. Diabetes Care, 2013. 36(7): p. 2001-8.

132. Chen, X., T.O. Scholl, M. Leskiw, et al., Differences in maternal circulating fatty acid composition and dietary fat intake in women with gestational diabetes mellitus or mild gestational hyperglycemia. Diabetes Care, 2010. 33(9): p. 2049-54.

133. Zhang, C., S. Liu, C.G. Solomon, et al., Dietary fiber intake, dietary glycemic load, and the risk for gestational diabetes mellitus. Diabetes Care, 2006. 29(10): p. 2223-30.

134. Bowers, K., D.K. Tobias, E. Yeung, et al., A prospective study of prepregnancy dietary fat intake and risk of gestational diabetes. Am. J. Clin. Nutr., 2012. 95(2): p. 446-53.

135. Behboudi-Gandevani, S., K. Safary, L. Moghaddam-Banaem, et al., The relationship between maternal serum iron and zinc levels and their nutritional intakes in early pregnancy with gestational diabetes. Biol. Trace Elem. Res., 2013. 154(1): p. 7-13.

136. Bowers, K., E. Yeung, M.A. Williams, et al., A prospective study of prepregnancy dietary iron intake and risk for gestational diabetes mellitus. Diabetes Care, 2011. 34(7): p. 1557-63.

137. Ley, S.H., A.J. Hanley, M. Sermer, et al., Lower dietary vitamin E intake during the second trimester is associated with insulin resistance and hyperglycemia later in pregnancy. Eur. J. Clin. Nutr., 2013. 67(11): p. 1154-6.

138. Zhang, C., M.A. Williams, I.O. Frederick, et al., Vitamin C and the risk of gestational diabetes mellitus: a case-control study. J. Reprod. Med., 2004. 49(4): p. 257-66.

139. Bao, W., K. Bowers, D.K. Tobias, et al., Prepregnancy low-carbohydrate dietary pattern and risk of gestational diabetes mellitus: a prospective cohort study. Am. J. Clin. Nutr., 2014.

140. Campbell, S.K., J. Lynch, A. Esterman, et al., Pre-pregnancy predictors of diabetes in pregnancy among Aboriginal and Torres Strait Islander women in North Queensland, Australia. Matern Child Health J, 2012. 16(6): p. 1284-92.

141. Chen, L., F.B. Hu, E. Yeung, et al., Prepregnancy consumption of fruits and fruit juices and the risk of gestational diabetes mellitus: a prospective cohort study. Diabetes Care, 2012. 35(5): p. 1079-82.

142. Bao, W., D.K. Tobias, F.B. Hu, et al., Pre-pregnancy potato consumption and risk of gestational diabetes mellitus: prospective cohort study. BMJ, 2016. 352: p. h6898.

143. Canda, M.T., O. Sezer, and N. Demir, An audit of seafood consumption awareness during pregnancy and its association with maternal and fetal outcomes in a Turkish population. J. Obstet. Gynaecol., 2011. 31(4): p. $293-7$.

144. Bao, W., D.K. Tobias, S.F. Olsen, et al., Pre-pregnancy fried food consumption and the risk of gestational diabetes mellitus: a prospective cohort study. Diabetologia, 2014.

145. Chen, L., F.B. Hu, E. Yeung, et al., Prospective study of pre-gravid sugar-sweetened beverage consumption and the risk of gestational diabetes mellitus. Diabetes Care, 2009. 32(12): p. 2236-41.

146. Hinkle, S.N., S.K. Laughon, J.M. Catov, et al., First trimester coffee and tea intake and risk of gestational diabetes mellitus: a study within a national birth cohort. BJOG, 2015. 122(3): p. 420-8.

147. Hu, F.B., Dietary pattern analysis: a new direction in nutritional epidemiology. Curr. Opin. Lipidol., 2002. 13(1): p. 39.

148. Kant, A.K., Indexes of overall diet quality: a review. J. Am. Diet. Assoc., 1996. 96(8): p. 785-91.

149. Kipnis, V., A.F. Subar, D. Midthune, et al., Structure of dietary measurement error: results of the OPEN biomarker study. Am. J. Epidemiol., 2003. 158(1): p. 14-21; discussion 22-6. 


\section{Chapter 1}

150. Prentice, R.L., Y. Mossavar-Rahmani, Y. Huang, et al., Evaluation and comparison of food records, recalls, and frequencies for energy and protein assessment by using recovery biomarkers. Am. J. Epidemiol., 2011. 174(5): p. 591-603.

151. Brouwer-Brolsma, E.M., M.T. Streppel, L. van Lee, et al., A National Dietary Assessment Reference Database (NDARD) for the Dutch Population: Rationale behind the Design. Nutrients, 2017. 9(10).

152. Lee, C., A.J. Dobson, W.J. Brown, et al., Cohort Profile: the Australian Longitudinal Study on Women's Health. Int. J. Epidemiol., 2005. 34(5): p. 987-91. 
General introduction 



\section{Chapter 2}

Development and evaluation of the Dutch Healthy Diet index 2015

Moniek Looman, Edith J.M. Feskens, Mariëlle de Rijk, Saskia Meijboom, Sander Biesbroek, Elisabeth H.M. Temme, Jeanne de Vries, Anouk Geelen

Published in Public Health Nutrition, volume 20, September 2017, pages 2289-2299 


\section{Abstract}

Objective: To update the Dutch Healthy Diet index, a measure of diet quality, to reflect adherence to the Dutch dietary guidelines 2015 and to evaluate against participants' characteristics and nutrient intakes with the score based on 24 hour recall (24hR) data and FFQ data.

Design: The Dutch Healthy Diet index 2015 (DHD15-index) consists of 15 components representing the 15 food-based Dutch dietary guidelines of 2015. Per component the score ranges between zero and ten, resulting in a total score between zero (no adherence) and 150 (complete adherence).

Setting: Wageningen area, the Netherlands, 2011-2013

Subjects: Data of 885 men and women, aged 20-70 years, participating in the longitudinal NQplus study, who filled out two $24 \mathrm{hR}$ and one FFQ was used.

Results: The mean \pm SD score of the DHD15-index was $68.7 \pm 16.1$ for men and $79.4 \pm 16.0$ for women. Significant inverse trends were found between the DHD15-index and BMI, smoking, and intakes of energy, total fat, and saturated fat. A positive trend was seen across the sex-specific quintiles of the DHD15-index score with energy-adjusted micronutrient intakes. Mean DHD15-index score of the FFQ data was 15.5 points higher compared to $24 \mathrm{hR}$ data, with a correlation coefficient of 0.56 between the scores. Observed trends of the DHD15-index based on FFQ with participant characteristics, macronutrient intake and energy-adjusted micronutrients were similar to those with the DHD15-index based on $24 \mathrm{hR}$.

Conclusions: The DHD15-index score assesses adherence to the Dutch dietary guidelines 2015 and indicates diet quality. The DHD15-index score can be based on 24hR data and on FFQ data. 


\section{Introduction}

The Dutch Healthy Diet (DHD) index is a measure of diet quality as it assesses adherence to the Dutch dietary guidelines published in 2006 by the Health Council of the Netherlands [1]. The DHD-index has been used in a variety of applications, including intervention monitoring and epidemiological research [2-4]. A higher DHD-index score has been associated with more nutrient-dense diets [5]and lower risk of mortality[6], but not with cardiovascular disease [7] or quality adjusted life years [8].

In 2015, the Health Council of the Netherlands published an updated version of the Dutch dietary guidelines, based upon the latest scientific evidence [9]. Where the Dutch dietary guidelines of 2006 consisted of food-based and nutrient-based guidelines (e.g. vegetables, fruit, fibre and saturated fat), the 2015 guidelines are completely food-based (e.g. whole grain products, red and processed meat, and fats and oils) [10]. Due to this revision of the Dutch Dietary Guidelines the DHD-index needed to be updated. Therefore, we developed the Dutch Healthy Diet 2015 (DHD15) index as a tool to measure adherence to the Dutch dietary guidelines of 2015. In this paper we describe the development of this new score and examine associations between the DHD15-index with participants' characteristics, and energy, macro- and micronutrient intakes based on 24 hour recall (24hR) data. Additionally, the DHD15-index score derived from FFQ data was compared to the DHD15-index score derived from the $24 \mathrm{hR}$ data to assess comparability of the index when based on different dietary assessment instruments.

\section{Methods}

\section{Study design and population}

The Nutrition Questionnaires plus (NQplus) study is a longitudinal study on diet and health in the general Dutch population. The NQplus study has been described elsewhere [11]. Briefly, between May 2011 and December 2013, a total of 2,048 men and women were included, all randomly selected inhabitants of the cities Wageningen, Renkum, Ede, Arnhem, and Veenendaal which are located in the central part of the Netherlands. Inclusion criteria were age between 20-70 years and being able to speak and write Dutch.

Baseline measurements consisted of dietary assessment (including supplement use) with multiple $24 \mathrm{hR}$ and FFQ, anthropometric measurements (including height and body weight measurements), a venepuncture, a 24-hour urine collection and general questionnaires (including age, sex, highest achieved education level (low: primary school, vocational or lower general secondary education, 
moderate: higher secondary education or intermediate vocational training, high: higher vocational education or university) and smoking (yes/no)). Participants with data of least two telephone administered $24 \mathrm{hR}$ and a baseline FFQ $(n=885)$ were included for the current analysis.

\section{Dietary assessment}

\section{4 hour recalls}

The $24 \mathrm{hR}$ was administered by means of a telephone interview by trained dietitians of the Division of Human Nutrition of Wageningen University. The dietitian made an unannounced phone call to the participant and asked about the foods and drinks consumed the previous day according to a standardized protocol based on the five-step multiple-pass method [12]. Recalls were at least one month apart and the first two completed 24hR were used for the present analyses. Energy and nutrient intakes were estimated using the 2011 Dutch food composition table [13].

\section{Food frequency questionnaire (FFQ)}

A 180 -item semi-quantitative FFQ was used to assess usual dietary intake and was previously evaluated for energy intake, macronutrients, dietary fibre and selected vitamins $[14,15]$. Answer categories for frequency questions ranged between 'not in this month' to '6-7 days/week', and portion sizes were estimated using natural portions (bread shapes) and commonly used household measures (e.g. spoon and cup). Average daily nutrient intakes were calculated by multiplying frequency of consumption by portion size and nutrient content per gram using the 2011 Dutch food composition table [13]. We estimated the same micronutrient intakes as for the $24 \mathrm{hR}$, except for iron and magnesium as the FFQ was not developed to estimate these intakes. The FFQ was administered online using the open-source survey tool Limesurvey.

\section{Development of the DHD15-index}

In Table 2.1 an overview of the components and their cut-off and threshold values can be found. For all 15 components a maximum of ten points could be allotted, resulting in a total score ranging from zero to 150 points. The components vegetables, fruit, legumes, nuts, fish, and tea are adequacy components, and the components red meat, processed meat, sweetened beverages and fruit juices, sodium and alcohol are moderation components. The component dairy is an optimum component with an optimal range of intakes, whereas the fats and oils component is defined as a ratio component to reflect replacement of intake of less desired foods with healthier options in that food group. The coffee component is defined as a qualitative component based on type of coffee. The component wholegrain is scored based on two subcomponents as there are two guidelines for grain products: an adequacy 


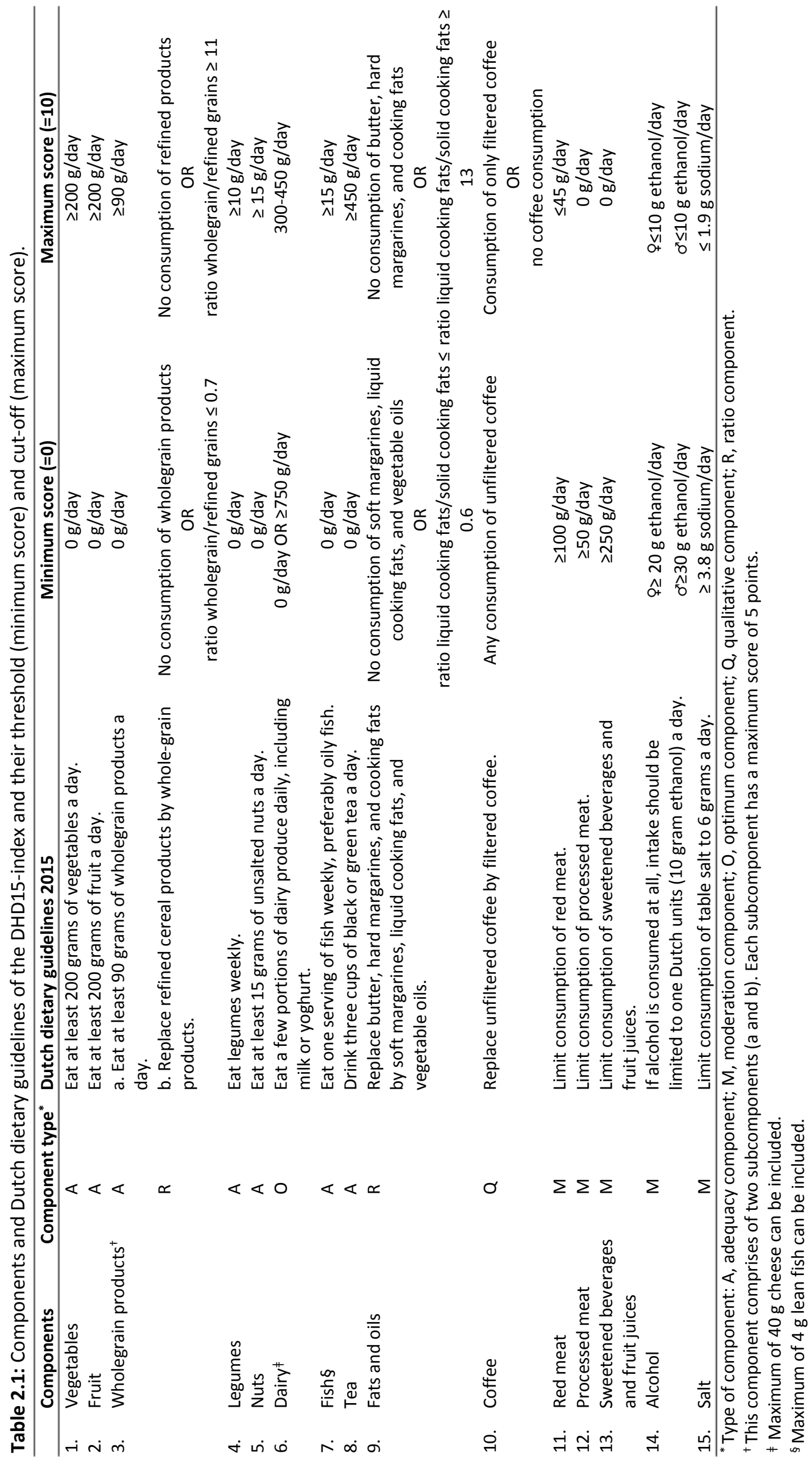


component for wholegrain consumption and a ratio component to reflect replacement of refined grain products by wholegrain products.

Cut-off values represent the minimum (for adequacy components) and maximum (for moderation components) required amount of consumption awarded with ten points. The threshold values represent the lowest level of intake awarded with zero points (for moderation components only). For adequacy components, no intake is awarded with zero points. Intakes between the cut-off and threshold value are scored proportionally. In the text below, for each guideline/component the included food groups, explanation of cut-off and threshold values and adaptations for estimation with an FFQ are discussed, if applicable. This is followed by an explanation of the scoring per type of component.

\section{Vegetables}

The first component is based on the recommendation to consume at least 200 grams of vegetables per day. Foods for this component are vegetables including frozen and canned vegetables, peas, and salads, but not legumes or potatoes. The cut-off was set at 200 grams as quantified in the guideline.

\section{Fruit}

The second component is based on the recommendation to consume at least 200 grams of fruit a day. Fresh fruit intake was included for this component, but not dried fruit as this has a relatively high energy and sugar content compared to fresh fruit [16]. In contrast to the previous DHD-index, fruit juices are no longer included. The cut-off was set at $\mathbf{2 0 0}$ grams as quantified in the guideline.

\section{Wholegrain products}

The third component was based on two guidelines regarding wholegrain foods and therefore scored with two subcomponents. The first subcomponent was based on the recommendation to consume at least 90 grams of wholegrain products per day. The cut-off was set at 90 grams as quantified in the guideline. The second subcomponent is based on the recommendation to replace refined cereal products by wholegrains products and is scored as a ratio component obtained by dividing intake of wholegrain products by intake of refined cereal products. There was no quantitative recommendation, nor information about the level of intake of refined cereal products associated with adverse health effects to base the cut-off or threshold value on. Therefore, we used the 15th percentile of the intake distribution of the Dutch reference population based on two day averages (Dutch National Food Consumption Survey 2007-2010 [17]) as (arbitrary) cut-off value. The threshold value was equal to the 85th percentile as we also did for the DHD-index. The maximum score for both subcomponents is five points. By adding the scores of the two subcomponents the score for wholegrain products is obtained. Included food groups were cereal products used as staple component of the diet (e.g. bread products, 
bread replacement products, muesli, pasta and rice), but no snacks made of cereal products such as biscuits. Foods were categorized as wholegrain product if they contained at least 25 per cent wholegrain flour; otherwise they were categorized as refined grain product.

\section{Legumes}

The fourth component is based on the recommendation to consume legumes weekly. As intakes in the score are expressed per day, one portion of legumes (60 grams [16]) was divided by seven and rounded to $10 \mathrm{~g} /$ day to obtain the cut-off value. Included food groups are pulses, lentils, beans and chickpeas, but not peas and peanuts [18].

Nuts

The fifth component assesses unsalted nut consumption and is based on the recommendation to consume at least 15 grams of unsalted nuts a day. The cut-off was set at 15 grams as quantified in the guideline. As stated in the guideline, only unsalted nuts were included in this component. However, the FFQ did not distinguish between unsalted and salted nuts. Therefore, total nut consumption was included for this component for the FFQ based DHD15-index score.

\section{Dairy}

The sixth component, dairy, is based on the recommendation to consume a few portions of dairy a day including milk and yoghurt. Included food groups are milk, milk products, yoghurt, cheese, cream, custard, and porridge prepared with dairy. This component was interpreted as an optimum component. Two to three portions a day, with a portion size of $150 \mathrm{~g}$ per portion (Netherlands Nutrition Centre), resulted in the optimum range of intake (300-450g/day) and a score of ten points. An intake of more than two times the average recommended amount was set as threshold value. Cheese intake was also included in total dairy intake, but limited to a maximum of $40 \mathrm{~g}$ (as set by the Netherlands Nutrition Centre) to account for differences in portion sizes between milk and cheese. Furthermore, a limitation in cheese intake ensures that the maximum score for dairy can only be obtained when milk or yoghurt products are consumed, as specified in the recommendation of the Health Council of the Netherlands.

\section{Fish}

The seventh component, fish, is based on the recommendation to consume one portion of fish a week, preferably oily fish. One portion of fish (100 grams [16]) was divided by seven and rounded to obtain the cut-off value of $15 \mathrm{~g} / \mathrm{d}$ for fish. As the recommendation favours intake of oily fish, a maximum of $4 \mathrm{~g} / \mathrm{d}$ of lean fish was included. This maximum was derived from the ratio three times oily fish and one time lean fish (per month) as set by the Netherlands Nutrition Centre. 
Tea

The eighth component is based on the recommendation to consume three cups of black or green tea a day. Portion sizes set by the Netherlands Nutrition Centre were used to arrive at a cut-off value of $450 \mathrm{~g} /$ day (equal to $450 \mathrm{ml} /$ day). As the FFQ does not distinguish between types of tea, total tea consumption was used for this component for the FFQ based DHD15-index score.

\section{Fats and oils}

The ninth component is a ratio component based on the recommendation for fats and oils. The ratio is obtained by dividing intake of soft margarines, liquid cooking fats and vegetable oils by intake of butter, hard margarines and cooking fats. Similar to the ratio subcomponent whole grains, cut-off and threshold values were derived from the 15th percentile and 85th percentile of the intake distribution of the Dutch reference population [17].

\section{Coffee}

The tenth component, coffee, is a quality component, based on type of coffee (filtered or unfiltered). Scoring for this component is, contrary to the other components, dichotomous. No consumption of unfiltered coffee or no consumption of coffee at all, was set as criterion for the maximum score of ten points, whereas any consumption of unfiltered coffee is awarded with zero points. Because both the 24hR and FFQ do not distinguish between types of coffee consumed, the component score for coffee could not be assessed in this study.

\section{Red meat}

The eleventh component, red meat, is based on the recommendation to limit red meat consumption. Included food products for this component are beef, pork, duck, pheasant, offal, and game products. The Netherlands Nutrition Centre advices to consume less than 300 grams of red meat per week (about $45 \mathrm{~g} /$ day). The cut-off value was thus set at an intake of $45 \mathrm{~g} /$ day. The Health Council of the Netherlands indicated that with a consumption of $100 \mathrm{~g} /$ day or more negative health effects were observed [19]. Consequently, the threshold value was set at an intake of $100 \mathrm{~g} / \mathrm{day}$.

\section{Processed meat}

The twelfth component, processed meat, is based on the recommendation to limit consumption of processed meat. Both processed red meat and processed white meat are food groups included in this component. As the Health Council of the Netherlands indicated that especially consumption of processed meat should be limited, the cut-off value was set at no consumption. The Health Council of the Netherlands indicated that negative health effects of processed meat are observed at intakes of $50 \mathrm{~g} /$ day or more [19], and therefore this was set as threshold value. 


\section{Sweetened beverages and fruit juices}

The thirteenth component was based on the recommendation to limit consumption of sweetened beverages and fruit juices. For this component, sugar-sweetened soft drinks, sugar-sweetened dairy drinks and fruit juices were included. No consumption was set as cut-off value and a consumption of $250 \mathrm{~g} /$ day as threshold value, as consumption of more than 250 grams per day was found to be associated with weight gain [20].

\section{Alcohol}

The fourteenth component, alcohol, is based on the recommendation to limit consumption to one Dutch unit (10 gram ethanol a day), if alcohol is consumed at all. This 10 gram ethanol per day was set as cut-off value. Negative health effects occur at different intakes for men and women [21], therefore the threshold value was differentiated by sex. For women an intake of 20 gram ethanol a day was associated with negative health effects, and thus this intake was used as threshold value, whereas for men this was set at 30 gram ethanol a day.

\section{Salt}

The last component, salt, is based on the recommendation to consume less than 6 grams of table salt a day. This corresponds to a recommended consumption of 2.4 grams of sodium per day or less. For this component the sodium content of all consumed foods was summed to obtain sodium intake per day. Ideally, salt consumption is assessed with sodium content based on a $24 \mathrm{~h}$ urine collection. However, this was not available for the Dutch reference population. Therefore, the threshold value is based on the intake distribution of the Dutch reference population assessed with two 24hR. With sodium intakes based on $24 \mathrm{hR}$ and especially $\mathrm{FFQ}$, salt added during cooking and the dinner table is not taken into account. The contribution of these sources is assumed to be on average $20 \%$ of total sodium intake in the Netherlands [22]. In most studies there is no data available on the amount of salt added during cooking and at the dinner table. Therefore we adjusted the cut-off value, representing the recommended maximum sodium intake, by $20 \%$ to compensate for this.

\section{Scoring}

For the adequacy components vegetables, fruit, nuts, legumes, nuts, fish, and tea the minimum score was given when there was no consumption of this component. Intakes equal to the cut-off value or higher were given the maximum score of ten points. The scores for the intake between zero and the cut-off value were calculated by dividing the reported intake by the cut-off value and subsequently multiplying the obtained ratio by ten (Figure 2.1a). For the moderation components red meat, processed meat, sweetened beverages and fruit juices, sodium and alcohol zero points were assigned if intake was above the threshold value. Ten points were assigned if intake was equal to or lower than 
the cut-off value. The scores for intake between threshold value and cut-off value were calculated by dividing the difference between the intake and the cut-off value by the difference between the threshold value and the cut-off value. This ratio was subsequently multiplied by ten. The obtained score was subtracted from ten to obtain the component score, as the score for moderation components has to decrease when intake increases (Figure 2.1b). The maximum score for the optimum component dairy was assigned if intake was within the given range. No consumption was scored with the minimum score of zero points. Intakes lower than the cut-off value were scored by dividing the reported intake by the lower cut-off value of the range and subsequently multiplying the obtained ratio by ten. Intakes between the higher cut-off value of the range and the threshold value were scored by dividing the difference between the intake and the cut-off value by the difference between the threshold value and the cut-off value. This ratio was subsequently multiplied by ten. The obtained score was subtracted from ten to ensure that the score decreases when intake increases. For intakes above the threshold value the minimum score of zero points was given (Figure 2.1c). Cut-off values and threshold values for the ratio components were set for the calculated ratios, instead of intakes. The maximum score of ten points was assigned if the ratio was higher than the cut-off value. The minimum score of zero points was assigned if the ratio was lower than the threshold value. Intakes between the cut-off and threshold value were calculated by diving the difference between the ratio and the threshold value by the difference between the cut-off and threshold value (Figure 2.1d).

\section{Statistical analysis}

All food, energy and nutrient intakes assessed by $24 \mathrm{hR}$ were averaged over two days before being used to score individual dietary intakes. DHD15-index scores reported in this paper are based on $24 \mathrm{hR}$ unless stated otherwise. Means across sex-specific quintiles of the DHD15-index score were tested using $\mathrm{P}$ for trend calculated with general linear models. Macro- and micronutrient intakes are reported with and without energy adjustment. Adjusted macronutrient intakes are presented as energy percentage (E\%) and adjusted micronutrient intakes are presented as mean intakes per 4.2MJ.

Concordance of ranking of participants with the DHD15-index scores based on 24hR data and FFQ data was studied by analysing correlations between the scores and cross-classification of quintiles. Partial correlation coefficients were calculated for the DHD15-index score and its components based on the $24 \mathrm{hR}$ data and the FFQ data, adjusting for energy intake assessed by the $24 \mathrm{hR}$. Pearson correlations were used for normally distributed variables and Spearman correlations for skewed variables. The $95 \%$ confidence intervals $(95 \% \mathrm{Cl})$ were calculated using Fisher's Z-transformation. The Wilcoxon signedrank test was used to test differences between medians. Cross-classification and Kendall's $\tau-b$ coefficient were used to assess agreement of participants' ranking for the DHD15-index score based 

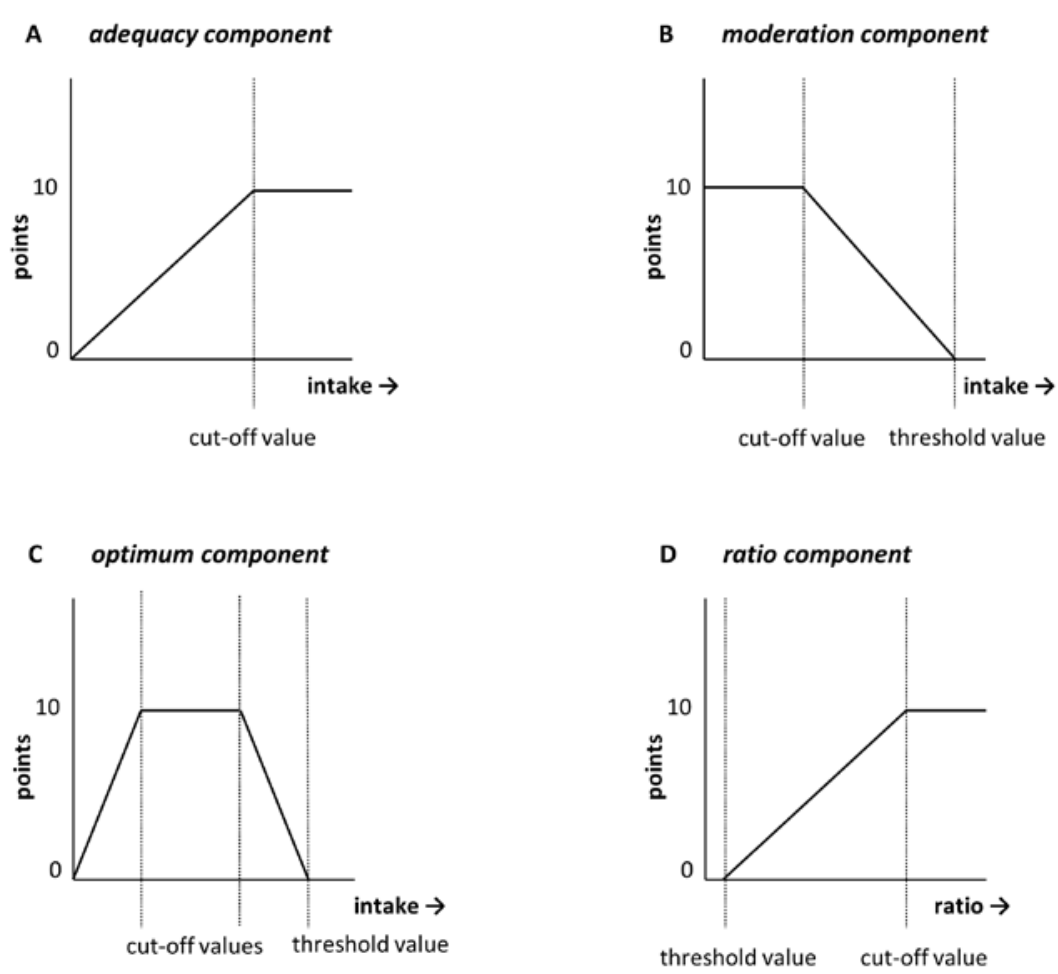

Figure 2.1: Graphic presentation of scoring for the DHD15-index for the different type of components: adequacy component (A), moderation component (B), optimum component (D), and ratio component (D).

on $24 \mathrm{hR}$ data and on FFQ data. All statistical analyses were performed using SAS 9.3 (SAS Institute Inc.) and a P value of $<0.05$ was considered statistically significant.

\section{Results}

Mean \pm SD age of the population was $54.0 \pm 11.7$ years and mean BMI was $25.9 \pm 4.0 \mathrm{~kg} / \mathrm{m}^{2}$. Fifty-three percent of the population was men. Men were significantly older $(56.4 \pm 10.8 y)$ than women $(51.4 \pm 12.1 \mathrm{y})$ and their BMI was significantly higher $(26.4 \pm 3.5 \mathrm{~kg} / \mathrm{m} 2)$ than that of the women $(25.4 \pm 4.5$ $\mathrm{kg} / \mathrm{m} 2$ ). More than $60 \%$ of the population completed a level of higher education and less than $10 \%$ of the population was current smoker, this did not differ between men and women.

The mean \pm SD DHD15-index score for the total population based on $24 \mathrm{hR}$ data was $73.7 \pm 16.9$ points (Table 2.2). The total DHD15-index score was significantly higher for women than for men (mean difference of 10.7 points). The total DHD15-index score was normally distributed and ranged from 24.3 to 126.2 points. The highest mean component score was that for the component red meat followed 
by alcohol, whereas the lowest mean component score was observed for the component legumes followed by fish. Women had significantly higher (i.e. better) scores for the components vegetables, fruit, nuts, tea, red meat, processed meat, sweetened beverages and juices, alcohol and salt. Men scored significantly higher on the component wholegrain products. The DHD15-index score was positively correlated with the DHD-index based on the dietary guidelines of $2006(\rho=0.62 ; p<0.001)$.

BMI and smoking were inversely associated with the DHD15-index (Table 2.3). Age and supplement use were positively associated with the DHD15-index score, whereas education was not associated with the index score. Participants with a higher DHD15-index score had s lower energy intake. Of the macronutrients, animal protein, added sugar, total fat and saturated fat were inversely associated whereas dietary fibre and vegetable protein were positively associated with the DHD15-index score, and these trends remained significant after energy adjustment $(p<0.05)$. For the micronutrients calcium, folate, iron, magnesium, potassium, vitamin B6 and vitamin C significant positive trends were observed across quintiles of the DHD15-score, both crude and after energy adjustment. Thiamine was inversely associated with the index score, but this association disappeared after energy adjustment. For the micronutrients riboflavin, vitamin $\mathrm{A}$, vitamin $\mathrm{B} 12$, and vitamin $\mathrm{E}$ significant positive associations were observed for the energy adjusted intakes, but not for the unadjusted intakes.

The mean DHD15-index score based on FFQ data was 15.5 points higher than based on $24 \mathrm{hR}$ data ( $p<0.001 ;$ Table 2.4; Supplemental Figure 2.1). The correlation between the DHD15-index scores based on $24 \mathrm{hR}$ and $\mathrm{FFQ}$ data was 0.58 (95\% $\mathrm{Cl} 0.53-0.62)$, also after energy adjustment $(0.56,95 \% \mathrm{Cl} 0.52-$ 0.61). The correlations between the component scores based on FFQ and $24 \mathrm{hR}$ data ranged between 0.14 and 0.65 . The lowest correlations were observed for the components legumes and red meat, while the highest correlations were seen for tea and alcohol. For most components mean component scores were higher based on FFQ data compared with $24 \mathrm{hR}$ data $(p<0.001)$. Largest differences in mean component score were seen for legumes (5.0 point difference) and fish (3.8 point difference). Results from cross-classification showed that $78 \%$ was classified in the same or neighbouring quintile and only $1 \%$ was classified in the opposite quintile, with Kendall's $\tau-b$ coefficient of 0.41 ( $95 \% \mathrm{Cl} 0.36-0.45$ ). 
Table 2.2: Mean (SD) scores of the DHD15-index components in 885 Dutch men and women aged 20-75 years*.

\begin{tabular}{lcccc}
\hline & Total & Men & Women & P-value between sex $^{\dagger}$ \\
\hline DHD15-index ${ }^{\ddagger}$ & $73.7(16.9)$ & $68.7(16.1)$ & $79.4(16.0)$ & $<0.001$ \\
Vegetables & $6.2(3.2)$ & $5.9(3.2)$ & $6.6(3.1)$ & 0.001 \\
Fruit & $6.2(3.7)$ & $5.8(3.8)$ & $6.7(3.5)$ & $<0.001$ \\
Wholegrain products & $5.7(2.7)$ & $6.0(2.5)$ & $5.5(2.8)$ & 0.006 \\
Legumes & $0.8(2.6)$ & $0.6(2.4)$ & $0.9(2.9)$ & 0.055 \\
Nuts & $2.6(3.9)$ & $2.4(3.9)$ & $2.8(3.9)$ & 0.023 \\
Dairy & $6.0(3.2)$ & $5.9(3.2)$ & $6.2(3.3)$ & 0.189 \\
Fish & $2.2(3.8)$ & $2.4(4.0)$ & $2.0(3.6)$ & 0.447 \\
Tea & $5.8(4.0)$ & $4.7(4.0)$ & $7.1(3.7)$ & $<0.001$ \\
Fats and oils & $6.2(4.5)$ & $6.2(4.5)$ & $6.1(4.5)$ & 0.881 \\
Red meat & $8.6(3.0)$ & $8.2(3.3)$ & $9.0(2.6)$ & $<0.001$ \\
Processed meat & $4.3(4.0)$ & $3.4(3.8)$ & $5.3(4.1)$ & $<0.001$ \\
Sweetened beverages and & $5.8(4.0)$ & $5.4(4.1)$ & $6.2(3.7)$ & 0.007 \\
fruit juices & $7.1(4.0)$ & $6.6(4.0)$ & $7.7(3.9)$ & $<0.001$ \\
Alcohol & $6.2(3.4)$ & $5.3(3.4)$ & $7.3(2.9)$ & $<0.001$ \\
Salt & & &
\end{tabular}

* Dietary intakes are based on average intake of two-24hR.

${ }^{+}$Independent $\mathrm{t}$-test comparing men and women for total DHD15-index score and Mann-Whitney $\mathrm{U}$ test comparing men and women for the individual component scores.

‡ DHD15-index score ranging from zero to 140 points.

The mean DHD15-index score based on FFQ was also significantly higher for women than men (mean difference 9.5 points). Significant differences between men and women in component scores based on FFQ were largely similar to the differences between men and women based on 24hR (Supplemental Table 2.1). Associations and trends observed across quintiles of DHD15-index score based on FFQ with participant characteristics, macronutrient intake and energy-adjusted micronutrients showed similar results to the results based on $24 \mathrm{hR}$ (Supplemental Table 2.2). The only exception was energy-adjusted vitamin B12 where a positive trend across quintiles of DHD15-index was observed based on 24hR data but not with FFQ data. 
Table 2.3: Distribution of characteristics, macronutrient intake and selected micronutrient intakes (means (SD)) across sex-specific quintiles of the DHD15-index in 885 Dutch men and women*.

\begin{tabular}{|c|c|c|c|c|c|c|}
\hline & \multicolumn{5}{|c|}{ Sex-specific quintiles DHD15-index } & \multirow[b]{2}{*}{$\begin{array}{l}\text { P for } \\
\text { trend }\end{array}$} \\
\hline & $\begin{array}{c}\mathrm{Q} 1 \\
\mathrm{~N}=178\end{array}$ & $\begin{array}{c}\mathrm{Q} 2 \\
\mathrm{~N}=178\end{array}$ & $\begin{array}{c}\mathrm{Q} 3 \\
\mathrm{~N}=175\end{array}$ & $\begin{array}{c}\mathrm{Q} 4 \\
\mathrm{~N}=177\end{array}$ & $\begin{array}{c}\text { Q5 } \\
\mathrm{N}=177\end{array}$ & \\
\hline Mean DHD15-index score & 51.8 & 64.0 & 72.7 & 80.8 & 96.0 & \\
\hline Mean DHD-index score ${ }^{\dagger}$ & $40.0(8.9)$ & $44.3(9.2)$ & $49.1(9.1)$ & $53.3(9.6)$ & $58.8(8.7)$ & $<0.001$ \\
\hline Age $(y)$ & $51.4(11.9)$ & $52.8(11.6)$ & $54.4(12.3)$ & $55.7(10.6)$ & $55.9(11.5)$ & $<0.001$ \\
\hline $\mathrm{BMI}\left(\mathrm{kg} / \mathrm{m}^{2}\right)$ & $26.7(4.4)$ & $26.3(4.4)$ & $25.9(3.9)$ & $25.8(3.8)$ & $25.0(3.2)$ & $<0.001$ \\
\hline Supplements (\%) & 34.3 & 41.0 & 40.6 & 46.3 & 50.1 & 0.001 \\
\hline Education $(\%)^{\ddagger}$ & & & & & & 0.346 \\
\hline Low & 7.3 & 5.1 & 9.8 & 5.1 & 3.4 & \\
\hline Middle & 29.9 & 29.4 & 24.9 & 32.7 & 29.4 & \\
\hline High & 63.7 & 65.5 & 65.3 & 62.2 & 67.2 & \\
\hline Smoking (\%) & 20.5 & 8.3 & 8.0 & 4.5 & 5.6 & $<0.001$ \\
\hline \multicolumn{7}{|l|}{ Macronutrient intake } \\
\hline Energy intake (MJ/day) & $9.3(2.4)$ & $8.9(2.1)$ & $8.8(2.2)$ & $8.7(2.1)$ & $8.6(1.9)$ & $<0.001$ \\
\hline Protein intake (g/day) & $84.8(25.6)$ & $81.9(21.7)$ & $81.3(21.1)$ & $83.6(22.4)$ & $81.1(17.0)$ & 0.281 \\
\hline Protein intake (E\%) & $15.6(3.1)$ & $15.8(2.7)$ & $16.0(3.1)$ & $16.7(3.0)$ & $16.5(2.9)$ & $<0.001$ \\
\hline $\begin{array}{l}\text { Vegetable protein intake } \\
\text { (g/day) }\end{array}$ & $32.4(11.1)$ & $32.9(10.3)$ & $33.2(10.8)$ & $33.7(10.6)$ & $37.1(11.2)$ & $<0.001$ \\
\hline Animal protein intake (g/day) & $52.2(21.5)$ & $49.0(18.3)$ & $48.0(17.1)$ & $50.0(19.6)$ & $44.1(14.3)$ & $<0.001$ \\
\hline Carbohydrate intake (g/day) & $236(70)$ & $225(66)$ & $224(62)$ & $225(63)$ & $222(58)$ & 0.077 \\
\hline Carbohydrate intake (E\%) & $43.3(7.3)$ & $43.2(7.1)$ & $43.8(7.0)$ & $44.6(6.8)$ & $44.4(6.5)$ & 0.035 \\
\hline Added sugar (g/day) & $53.3(31.0)$ & $47.2(26.9)$ & $47.0(28.1)$ & $43.2(27.6)$ & $37.7(23.0)$ & $<0.001$ \\
\hline Fibre (g/day) & $19.8(6.6)$ & $21.4(6.9)$ & $22.5(6.7)$ & $24.4(6.9)$ & $27.0(7.7)$ & $<0.001$ \\
\hline Total fat intake (g/day) & $86.6(26.8)$ & $82.4(24.7)$ & $80.7(26.5)$ & $78.0(25.8)$ & $77.7(23.7)$ & $<0.001$ \\
\hline Total fat intake (E\%) & $33.9(5.4)$ & $33.9(5.3)$ & $33.4(5.8)$ & $32.8(5.7)$ & $33.1(5.8)$ & 0.042 \\
\hline Saturated fat intake (g/day) & $32.9(10.7)$ & $30.6(10.5)$ & $30.0(10.8)$ & $28.5(10.4)$ & $26.4(9.0)$ & $<0.001$ \\
\hline Saturated fat intake (E\%) & $13.1(3.0)$ & $12.7(3.0)$ & $12.6(2.9)$ & $12.2(3.0)$ & $11.3(2.7)$ & $<0.001$ \\
\hline \multicolumn{7}{|l|}{ Micronutrient intake per 4.2} \\
\hline \multicolumn{7}{|l|}{ MJ (day) } \\
\hline Calcium (mg) & 435 (144) & $456(141)$ & $505(163)$ & $529(174)$ & $538(147)$ & $<0.001$ \\
\hline Folate (mcg) & $115(38)$ & $130(42)$ & $143(59)$ & $152(50)$ & $169(51)$ & $<0.001$ \\
\hline Iron (mg) & $5.1(1.1)$ & $5.2(1.0)$ & $5.5(1.4)$ & $5.6(1.2)$ & $5.9(1.4)$ & $<0.001$ \\
\hline Magnesium (mg) & $152(31)$ & $166(25)$ & $174(35)$ & $186(33)$ & $197(36)$ & $<0.001$ \\
\hline Potassium (mg) & $1473(294)$ & $1568(269)$ & 1641 (389) & $1731(344)$ & 1779 (329) & $<0.001$ \\
\hline Riboflavin (mg) & $0.67(0.22)$ & $0.68(0.19)$ & $0.71(0.22)$ & $0.76(0.24)$ & $0.74(0.19)$ & $<0.001$ \\
\hline Thiamine (mg) & $0.48(0.19)$ & $0.48(0.15)$ & $0.47(0.14)$ & $0.48(0.14)$ & $0.48(0.13)$ & 0.957 \\
\hline Vitamin A (RE) & $455(380)$ & $446(338)$ & $450(297)$ & $495(323)$ & 534 (496) & 0.019 \\
\hline Vitamin B6 (mcg) & $697(377)$ & 755 (318) & $763(354)$ & $854(367)$ & $882(367)$ & $<0.001$ \\
\hline Vitamin B12 (mcg) & $2.3(1.9)$ & $2.1(1.3)$ & $2.2(2.1)$ & $2.6(2.0)$ & $2.5(1.5)$ & 0.030 \\
\hline Vitamin C (mg) & $42(27)$ & $44(28)$ & $48(31)$ & $53(31)$ & $56(32)$ & $<0.001$ \\
\hline Vitamin E (mg) & $5.8(2.5)$ & $6.1(2.3)$ & $6.1(2.0)$ & $6.2(2.0)$ & $6.8(2.5)$ & $<0.001$ \\
\hline
\end{tabular}

$\mathrm{RE}$, retinol equivalents

* Dietary intakes are based on average intake of two-24hR.

${ }^{+}$Dutch Healthy Diet (index) score based on 8 components without components physical activity and acidic foods and drinks, with a total score ranging from zero (no adherence to Dutch dietary guidelines 2006) to 80 (maximum adherence). ₹Low education=primary school, vocational and lower general secondary education. Moderate=higher secondary education and intermediate vocational training. High=higher vocational education and university. 
Table 2.4: Mean (SD) of the DHD15-index and its component scores based on two $24 \mathrm{hR}$ and on a FFQ in 885 Dutch men and women and partial correlation scores $(95 \% \mathrm{Cl})$ between the two scores.

\begin{tabular}{|c|c|c|c|c|}
\hline & $24 \mathrm{hR}$ & FFQ & & \\
\hline & Mean (SD) & Mean (SD) & Correlation $^{*}$ & $95 \% \mathrm{Cl}$ \\
\hline DHD15-index ${ }^{\dagger}$ & 73.7 (16.9) & $89.2(15.4)$ & 0.56 & $0.52,0.61$ \\
\hline 1.Vegetables & $6.2(3.2)$ & $6.8(2.7)$ & 0.33 & $0.27,0.39$ \\
\hline 2.Fruit & $6.2(3.7)$ & $7.1(3.4)$ & 0.55 & $0.50,0.59$ \\
\hline 3.Wholegrain products & $5.7(2.7)$ & $5.8(2.0)$ & 0.32 & $0.26,0.38$ \\
\hline 4.Legumes & $0.8(2.6)$ & $5.8(4.5)$ & 0.14 & $0.07,0.20$ \\
\hline 5.Nuts & $2.6(3.9)$ & $3.9(3.6)$ & 0.32 & $0.26,0.37$ \\
\hline 6.Dairy & $6.0(3.2)$ & $6.5(3.1)$ & 0.29 & $0.23,0.35$ \\
\hline 7.Fish & $2.2(3.8)$ & $6.1(3.1)$ & 0.26 & $0.19,0.32$ \\
\hline 8.Tea & $5.8(4.0)$ & $3.6(3.5)$ & 0.65 & $0.61,0.69$ \\
\hline 9.Fats and oils & $6.2(4.5)$ & $6.8(4.0)$ & 0.33 & $0.27,0.39$ \\
\hline 10.Red meat & $8.6(3.0)$ & $9.1(2.1)$ & 0.16 & $0.10,0.23$ \\
\hline 11.Processed meat & $4.3(4.0)$ & $6.0(3.2)$ & 0.40 & $0.34,0.45$ \\
\hline $\begin{array}{l}\text { 12.Sweetened beverages } \\
\text { and fruit juices }\end{array}$ & $5.8(4.0)$ & $6.4(3.4)$ & 0.51 & $0.46,0.56$ \\
\hline 13.Alcohol & $7.1(4.0)$ & $7.7(3.6)$ & 0.60 & $0.56,0.64$ \\
\hline 14.Sodium & $6.2(3.4)$ & $7.9(2.7)$ & 0.21 & $0.14,0.27$ \\
\hline
\end{tabular}

* Adjusted for energy intake as assessed by $24 \mathrm{hR}$.

${ }^{+}$DHD15-index score ranging from zero to 140 points.

Only total DHD15 score was normally distributed (Pearson correlation)

\section{Discussion}

The DHD15-index score assesses adherence to the Dutch dietary guidelines 2015 and is able to rank participants according to their adherence as was reflected by the variation in scores of the individual components of the index and the normally distributed total score. The index was positively associated with age, supplement use, fibre intake, and nutrient density and inversely associated with BMI, energy, total and saturated fat intake. The most pronounced differences in the score based on FFQ compared with $24 \mathrm{hR}$ data were found in the components reflecting episodically consumed foods such as fish, but associations of the DHD15-index with participants' characteristics and nutrient intakes were similar for the scores based on $24 \mathrm{hR}$ and on FFQ data.

The DHD15-index score was developed as an update of the previously developed DHD-index reflecting adherence to the Dutch dietary guidelines of 2006. Therefore we kept the design aspects of this updated score similar to the DHD-index: the different components reflected the guidelines as close as possible, for each component a minimum score of zero and a maximum score of ten points could be allotted, and intakes between the minimum and maximum were scored proportionally. Contrary to the dietary guidelines of 2006, the guidelines of 2015 are formulated in terms of foods [10]. Only foods 
and food groups with sufficient scientific evidence for an effect on chronic diseases were included in the dietary guidelines. A consequence of this approach is that the dietary guidelines do not cover the complete dietary intake and recommended intakes in the guidelines apply to the general population. However, subgroups might benefit from a higher or lower intake of a food group to meet specific nutrient recommendations. For example, vegetarians might benefit from a higher legume or nut intake to meet recommended protein intake. This applies to indices based on both foods and nutrients as well such as the Healthy Eating Index-2010. It should also be noted that within food-groups there is still room for discussion whether all foods within that food group should be included. For example, for dairy it could be argued that only low-fat dairy should be included as some studies show more favourable health effects for low-fat dairy compared to total dairy [23, 24]. However, as the evidence is ambiguous and the debate is still ongoing, the Health Council of the Netherlands decided to set the guideline for total dairy [9] and we stayed as close as possible to the guidelines.

To quantify the guidelines additional information from additional documents from the Health Council of the Netherlands (background documents) [18-21] and interpretation by experts was sometimes necessary. The evidence regarding intake levels at which adverse health effects occur, as described in the background documents, was used to set threshold values (i.e. the intake that deserves zero points). This could be done for the moderation components red meat, processed meat, sweetened beverages and fruit juices, and alcohol. For example, according to the background document an intake of $250 \mathrm{~g}$ sweetened beverages and fruit juices a day is associated with an increased risk of weight gain and therefore the threshold value for this component was set at $250 \mathrm{~g} /$ day. For alcohol, adverse health effects associated with alcohol intake occur at different intake levels for men and women, therefore we set a different threshold values for men and women. For the components salt and the ratio components wholegrain products and fats and oils there was not enough information to set an evidence-based threshold value. For those components the threshold value was based on the 85th percentile of the intake distribution based on two day averages of the Dutch reference population [17]. This was also done for the DHD-index and is comparable to other indices such as the Healthy Eating Index-2010 [25]. For the ratio components the cut-off value was based on the 15th percentile of this intake distribution as there was no information on the ratio that deserves the maximum score. We used the information regarding standard portion sizes of the Netherlands Nutrition Centre [16] for the components legumes, dairy, fish, tea and red meat, as the guidelines do not specify the recommended intake or only in number of servings.

The DHD15-index score based on $24 \mathrm{hR}$ data showed a moderate correlation $(0.56,95 \% \mathrm{Cl} 0.52-0.61)$ with the DHD15-index score based on FFQ data and is comparable with the correlation found for the initial DHD-index $(0.48,95 \% \mathrm{Cl} 0.33-0.61)$ [1]. Ranking of participants showed moderate agreement as 
shown by Kendall's $\tau$-b coefficient and fairly good concordance as $78 \%$ was ranked in the same or neighbouring quintile and only 1 percent in the opposite quintile. The cut-off and threshold values for the scores are absolute levels of intakes. We assume that a $24 \mathrm{hR}$ is more suitable to estimate dietary intakes on a group level as the used FFQ is designed to cover at least $90 \%$ of energy intake, but not able to capture $100 \%$ of the total intake [14]. However, several components are episodically consumed foods including fish, legumes and nuts. Using two-day averages of the $24 \mathrm{hR}$ can result in excess zeros and maximum scores, whereas an FFQ is better able to assess usual intakes of these episodically consumed foods because it assesses foods eaten during a longer period of time. This could be one explanation for the low correlations seen for these components when comparing the component score based on $24 \mathrm{hR}$ and FFQ data. Other reasons for the low correlations between the scores based on the two methods are the biases inherent to the $24 \mathrm{hR}$ (e.g. high day-to-day variability) and FFQ (e.g. aggregation of food items, standard portion sizes) [26]. Surprisingly, also red meat showed a very low correlation, and appeared to be an episodically consumed food in this health conscious population. Mean intakes were substantially lower than the intake of the Dutch reference population, which could not be explained by a difference in number of non-consumers. A possible alternative to better estimate these episodically consumed foods would be using $24 \mathrm{hR}$ with an additional short propensity questionnaire as also advised for surveillance [27]. Additionally, the FFQ used in the present study was not able to distinguish between unsalted and salted nuts, and between types of tea, whereas the $24 \mathrm{hR}$ is able to make these distinctions. Both methods were not able to distinguish between types of coffee (filtered vs. unfiltered). In future studies, an adapted FFQ able to distinguish between types of nuts, tea and coffee as well as an adapted $24 \mathrm{hR}$ to assess type of coffee should be used.

Other limitations of the used dietary assessment methods should also be considered. Firstly, for both the $24 \mathrm{hR}$ and the FFQ it is known that estimates for salt intake are biased and usually underestimated because information on salt added during cooking and at the dinner table is lacking. By reducing the cut-off level with $20 \%$ we tried to adjust for this, but realize this decreases variation between people and thus results on the sodium component should be interpreted with caution. Ideally, sodium intake is estimated based on 24-hour urinary nitrogen, which is considered the gold standard for estimating sodium intake [28]. Secondly, for alcohol intake it was not possible to assess binge drinking with only two $24 \mathrm{hR}$ and the used FFQ. Finally, a difficulty arises in the handling of mixed foods (i.e. foods consisting of several (types of) ingredients). These mixed foods were broken down into their ingredients as coded by the Dutch Food Composition Table [13] and the individual ingredients were used in the calculation of the food intakes. However, some foods in the Dutch Food Composition Table still consist of several different ingredients from different food groups. For these mixed foods, we included the food if more than half of the weight of the mixed food consisted of a food group of one 
of the components. For example, vegetables on pizza (component vegetables), sausage roll (processed meat) and pea soup (legumes) are not included in calculation of food intake, but porridge is ( $\geq 50 \%$ dairy).

The DHD15-index score presented in this study was designed to capture the dietary pattern of the general Dutch population. The components of the DHD15-index are familiar components of Western dietary patterns and similar to components of several well-known and much used indices such as the Healthy Eating Index-2010 (vegetables, fruit, grains, dairy, alcohol, salt, fats and oils, and the components meat, fish, nuts and legumes in the protein foods) [25] and the Mediterranean Diet Score (vegetables, fruits, legumes, cereals, dairy, meat, alcohol, and fats and oils) [29]. However, it might be that for ethnic minorities with non-Dutch eating habits the score is limited in its use [30]. Furthermore, we evaluated the DHD15-index score in a population that might not be representative of the general Dutch population, as participants had in general a high level of education. Also, the high proportion of supplement users could indicate that this population is health conscious. Therefore, we used the intake distribution of the Dutch reference population [17] for the intake-based threshold values in the development of the score instead of the intake distribution of our study population. This also has the advantage that results of future studies can be compared as the same threshold values are used.

Evaluation of indices is necessary to establish whether an index is suitable for further use. In this study, several types of evaluation were carried out. First of all, we examined the relationship between the DHD15-index with nutrient intakes and observed positive trends between the DHD15-index score and energy-adjusted micronutrient intakes. This indicates that participants with higher DHD15-index scores have a more nutrient dense diet. We also observed an inverse association with total energy intake, total fat intake and saturated fat intake, and a positive relationship with fibre intake, also suggesting that a higher DHD15-index score indicates a healthier diet. Secondly, construct validity was examined by assessing the relationship between the DHD15-index score and participants' characteristics such as age, educational level and supplement use. Although the trend observed was not significant, the proportion of highly educated participants increased with higher DHD15-index scores whereas the proportion of lower educated participants decreased. Additionally, the supplement users, older participants, and participants with a lower BMI had higher DHD15-index scores. Also, the variation in total score and the individual component scores indicate discriminative power of the DHD15-index. Lastly, comparability of the index based on different dietary assessment instruments was satisfactory. In addition, we saw an acceptable correlation between the DHD15-index score and the previously validated DHD-index score based on the 2006 guidelines. Based on these observations we think that the DHD15-index is a good measure of diet quality. Further evaluation steps include assessing the relationship between the DHD15-index and chronic diseases and mortality and its ability 
to monitor trends in dietary intake over time. As energy intake and BMI are inversely associated with the DHD15-index score, energy adjustment should be considered when studying diet-disease associations, to be able to distinguish between effects from energy intake and the effects of diet quality as reflected by the DHD15-index score.

\section{Conclusions}

The DHD15-index score assesses adherence to the Dutch dietary guidelines of 2015 and is an indicator of diet quality as it is positively associated with nutrient density. Both $24 \mathrm{hR}$ and FFQ data can be used to assess the DHD15-index score resulting in some differences in individual components but an acceptable correlation between the total scores. In future research, the DHD15-index score can be used to study associations between diet quality and chronic diseases.

Compliance with ethical standards: The NQplus study was approved by the medical ethical committee of Wageningen University, and was conducted according to the guidelines of the declaration of Helsinki. All participants gave written informed consent before the start of the study.

Conflicts of interest: The authors declare that they have no conflict of interest. 


\section{References}

1. van Lee, L., A. Geelen, E.J. van Huysduynen, et al., The Dutch Healthy Diet index (DHD-index): an instrument to measure adherence to the Dutch Guidelines for a Healthy Diet. Nutr. J., 2012. 11: p. 49.

2. Sluik, D., L. van Lee, A. Geelen, et al., Alcoholic beverage preference and diet in a representative Dutch population: the Dutch national food consumption survey 2007-2010. Eur. J. Clin. Nutr., 2014. 68(3): p. 287-94.

3. Struijk, E.A., J.W. Beulens, A.M. May, et al., Dietary patterns in relation to disease burden expressed in DisabilityAdjusted Life Years. Am. J. Clin. Nutr., 2014. 100(4): p. 1158-65.

4. Struijk, E.A., A.M. May, J.W. Beulens, et al., Adherence to the Dutch Guidelines for a Healthy Diet and cancer risk in the European Prospective Investigation into Cancer and Nutrition-Netherlands (EPIC-NL) cohort. Public Health Nutr., 2014. 17(11): p. 2546-53.

5. van Lee, L., E.J. Feskens, E.J. Hooft van Huysduynen, et al., The Dutch Healthy Diet index as assessed by $24 \mathrm{~h}$ recalls and FFQ: associations with biomarkers from a cross-sectional study. Journal of nutritional science, 2013. 2: p. e40.

6. van Lee, L., A. Geelen, J.C. Kiefte-de Jong, et al., Adherence to the Dutch dietary guidelines is inversely associated with 20-year mortality in a large prospective cohort study. Eur. J. Clin. Nutr., 2016. 70(2): p. 262-8.

7. Struijk, E.A., A.M. May, N.L. Wezenbeek, et al., Adherence to dietary guidelines and cardiovascular disease risk in the EPIC-NL cohort. Int. J. Cardiol., 2014. 176(2): p. 354-9.

8. Fransen, H.P., J.W. Beulens, A.M. May, et al., Dietary patterns in relation to quality-adjusted life years in the EPICNL cohort. Prev. Med., 2015. 77: p. 119-24.

9. Health Council of the Netherlands, Dutch dietary guidelines 2015. Publication no. 2015/24. 2015, Health Council of the Netherlands: The Hague.

10. Kromhout, D., C.J. Spaaij, J. de Goede, et al., The 2015 Dutch food-based dietary guidelines. Eur. J. Clin. Nutr., 2016. 70(8): p. 869-78.

11. van Lee, L., A. Geelen, E.J. Hooft van Huysduynen, et al., Associations between company at dinner and daily diet quality in Dutch men and women from the NQplus study. Eur. J. Clin. Nutr., 2016. 70(12): p. 1368-1373.

12. Conway, J.M., L.A. Ingwersen, B.T. Vinyard, et al., Effectiveness of the US Department of Agriculture 5-step multiplepass method in assessing food intake in obese and nonobese women. Am. J. Clin. Nutr., 2003. 77(5): p. 1171-8.

13. NEVO-tabel, Dutch Food Composition Table 2011/version 3. 2011, National Institute for Publich Health and the Environment/Netherlands Nutrition Centre.

14. Siebelink, E., A. Geelen, and J.H. de Vries, Self-reported energy intake by FFQ compared with actual energy intake to maintain body weight in 516 adults. Br. J. Nutr., 2011. 106(2): p. 274-81.

15. Streppel, M.T., J.H. de Vries, S. Meijboom, et al., Relative validity of the food frequency questionnaire used to assess dietary intake in the Leiden Longevity Study. Nutr. J., 2013. 12: p. 75.

16. Netherlands Nutrition Centre, Richtlijnen Schijf van Vijf 2016, Netherlands Nutrition Centre: The Hague.

17. van Rossum CTM, F.H., Verkaik-Kloosterman J, Buurma-Rethans EJM, Ocké MC., Dutch National Food Consumption Survey 2007-2010: Diet of children and adults 7 to 69 years. 2011, National Institute for Public Health and the Environment: Bilthoven.

18. Health Council of the Netherlands, Dutch dietary guidelines 2015 - Background document legumes. Publication no. A15/18. 2015, Health Council of the Netherlands: The Hague.

19. Health Council of the Netherlands, Dutch dietary guidelines 2015 - Background document meat. Publication no. A15/27. 2015, Health Council of the Netherlands: The Hague.

20. Health Council of the Netherlands, Dutch dietary guidelines 2015 - Background document sugar-sweetened beverages. Publication no. A15/08. 2015, Health Council of the Netherlands: The Hague.

21. Health Council of the Netherlands, Dutch dietary guidelines 2015 - Background document alcohol. Publication no. A15/05. 2015, Health Council of the Netherlands: The Hague.

22. van Rossum CTM, B.-R.E., Fransen HP, Verkaik-Kloosterman J, Hendriksen MAH, Zoutconsumptie van kinderen en volwassenen in Nederland Resultaten uit de Voedselconsumptiepeiling 2007-2010 2012, National Institute for Public Health and the Environment: Bilthoven.

23. Aune, D., T. Norat, P. Romundstad, et al., Dairy products and the risk of type 2 diabetes: a systematic review and dose-response meta-analysis of cohort studies. Am. J. Clin. Nutr., 2013. 98(4): p. 1066-83.

24. Dong, J.Y., L. Zhang, K. He, et al., Dairy consumption and risk of breast cancer: a meta-analysis of prospective cohort studies. Breast Cancer Res. Treat., 2011. 127(1): p. 23-31.

25. Guenther, P.M., K.O. Casavale, J. Reedy, et al., Update of the Healthy Eating Index: HEl-2010. J. Acad. Nutr. Diet., 2013. 113(4): p. 569-80.

26. Willett WC, Nutritional Epidemiology. 3rd ed. 2013, New York: Oxford University Press.

27. de Boer, E.J., N. Slimani, P. van 't Veer, et al., The European Food Consumption Validation Project: conclusions and recommendations. Eur. J. Clin. Nutr., 2011. 65 Suppl 1: p. S102-7.

28. Brown, I.J., I. Tzoulaki, V. Candeias, et al., Salt intakes around the world: implications for public health. Int. J. Epidemiol., 2009. 38(3): p. 791-813.

29. Trichopoulou, A., T. Costacou, C. Bamia, et al., Adherence to a Mediterranean diet and survival in a Greek population. N. Engl. J. Med., 2003. 348(26): p. 2599-608.

30. Dekker, L.H., M. Nicolaou, R.M. van Dam, et al., Socio-economic status and ethnicity are independently associated with dietary patterns: the HELIUS-Dietary Patterns study. Food Nutr. Res., 2015. 59: p. 26317. 


\section{Supplementary material}

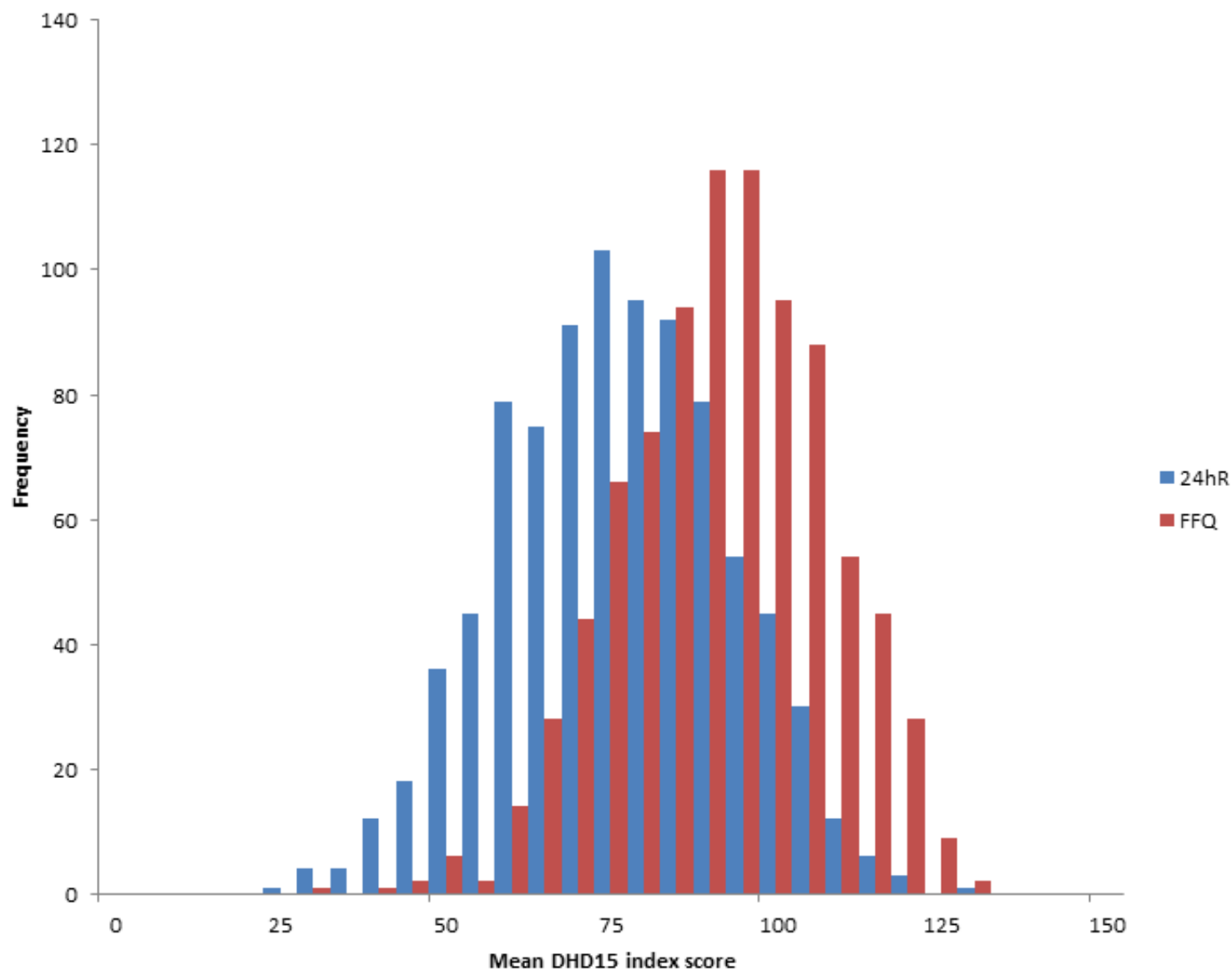

Supplemental Figure 2.1: Histogram of the mean Dutch Healthy Diet 2015 (DHD15) index score based on 2 24hR and based on FFQ data in 885 Dutch men and women. Mean (SD) DHD15-index score based on 2 24hR was 73.7 (16.9) points and mean (SD) score based on FFQ data was 89.2 (15.4) points. 
Supplemental Table 2.1: Mean (SD) scores of the DHD15-index components based on FFQ data in 885 Dutch men and women aged $20-75$ years.

\begin{tabular}{|c|c|c|c|c|}
\hline & Total & Men & Women & $\begin{array}{c}\text { P-value between } \\
\text { sex* }\end{array}$ \\
\hline DHD15-index ${ }^{\dagger}$ & $89.2(15.4)$ & $84.8(15.4)$ & $94.3(13.8)$ & $<0.001$ \\
\hline 1. Vegetables & $6.8(2.7)$ & $6.4(2.7)$ & $7.2(2.6)$ & $<0.001$ \\
\hline 2. Fruit & $7.1(3.4)$ & $6.6(3.6)$ & $7.7(3.2)$ & $<0.001$ \\
\hline 3. Wholegrain products & $5.8(2.0)$ & $5.8(2.0)$ & $5.7(2.0)$ & 0.463 \\
\hline 4. Legumes & $5.8(4.5)$ & $6.0(4.5)$ & $5.5(4.4)$ & 0.046 \\
\hline 5. Nuts & $3.9(3.6)$ & $3.7(3.6)$ & $4.1(3.7)$ & 0.083 \\
\hline 6. Dairy & $6.5(3.1)$ & $6.3(3.1)$ & $6.6(3.0)$ & 0.097 \\
\hline 7. Fish & $6.1(3.1)$ & $6.2(3.0)$ & $5.9(3.2)$ & 0.107 \\
\hline 8. Tea & $3.6(3.5)$ & $2.8(3.2)$ & $4.4(3.7)$ & $<0.001$ \\
\hline 9. Fats and oils & $6.8(4.0)$ & $6.9(4.0)$ & $6.8(4.0)$ & 0.843 \\
\hline 10. Red meat & $9.1(2.1)$ & $8.8(2.3)$ & $9.4(1.7)$ & $<0.001$ \\
\hline 11. Processed meat & $6.0(3.2)$ & $5.1(3.3)$ & $6.9(2.7)$ & $<0.001$ \\
\hline $\begin{array}{l}\text { 12. Sweetened beverages } \\
\text { and fruit juices }\end{array}$ & $6.4(3.4)$ & $5.9(3.4)$ & $7.0(3.2)$ & $<0.001$ \\
\hline 13. Alcohol & $7.7(3.6)$ & $7.1(3.7)$ & $8.4(3.3)$ & $<0.001$ \\
\hline 14. Salt & $7.9(2.7)$ & $7.1(3.0)$ & $8.7(2.0)$ & $<0.001$ \\
\hline
\end{tabular}

* Independent t-test comparing men and women for total DHD15-index score and Mann-Whitney $\mathrm{U}$ test comparing men and women for the individual component scores.

${ }^{+}$DHD15-index score ranging from zero to 140 points. 
Supplement Table 2.2: Distribution of characteristics, macronutrient intake and selected micronutrient intakes (means (SD)) across sex-specific quintiles of the DHD15-index based on FFQ data in 885 Dutch men and women.

\begin{tabular}{|c|c|c|c|c|c|c|}
\hline & \multicolumn{5}{|c|}{ Sex-specific quintiles DHD15-index based on FFQ data } & \multirow[b]{2}{*}{$\begin{array}{l}P \text { for } \\
\text { trend }\end{array}$} \\
\hline & $\begin{array}{c}\mathrm{Q} 1 \\
\mathrm{~N}=178\end{array}$ & $\begin{array}{c}\mathrm{Q} 2 \\
\mathrm{~N}=176\end{array}$ & $\begin{array}{c}\mathrm{Q} 3 \\
\mathrm{~N}=178\end{array}$ & $\begin{array}{c}\mathrm{Q} 4 \\
\mathrm{~N}=177\end{array}$ & $\begin{array}{c}\text { Q5 } \\
\mathrm{N}=176\end{array}$ & \\
\hline DHD15-index score ${ }^{*}$ & 68.8 & 81.2 & 89.6 & 97.0 & 109.7 & \\
\hline Mean DHD-index score ${ }^{\dagger}$ & $50.3(9.1)$ & $53.1(10.7)$ & $56.0(9.4)$ & $58.4(9.4)$ & $61.2(8.7)$ & $<0.001$ \\
\hline Age $(y)$ & $50.5(12.6)$ & $52.8(12.6)$ & $55.9(10.7)$ & $55.0(10.8)$ & $56.0(10.7)$ & $<0.001$ \\
\hline $\mathrm{BMI}\left(\mathrm{kg} / \mathrm{m}^{2}\right)$ & $26.4(4.3)$ & $26.3(4.7)$ & $25.9(3.8)$ & $25.8(3.7)$ & $25.1(3.3)$ & $<0.001$ \\
\hline Supplements (\%) & 34.3 & 44.9 & 36.0 & 44.1 & 54.0 & 0.001 \\
\hline \multicolumn{7}{|l|}{ Education $(\%)^{\ddagger}$} \\
\hline Low & 8.0 & 9.0 & 5.7 & 4.6 & 3.4 & 0.070 \\
\hline Middle & 30.1 & 27.8 & 30.0 & 27.8 & 30.7 & \\
\hline High & 61.9 & 63.1 & 64.4 & 67.6 & 65.9 & \\
\hline Smoking (\%) & 16.9 & 8.5 & 9.6 & 8.5 & 3.3 & $<0.001$ \\
\hline \multicolumn{7}{|l|}{ Macronutrient intake } \\
\hline Energy intake (MJ/day) & $9.1(3.1)$ & $8.9(2.7)$ & $8.5(2.3)$ & $8.4(2.2)$ & $8.4(1.9)$ & 0.001 \\
\hline Protein intake (g/day) & $76.5(22.4)$ & $77.6(22.4)$ & $73.8(18.4)$ & $73.0(17.2)$ & $75.1(16.2)$ & 0.116 \\
\hline Protein intake (E\%) & $14.6(2.8)$ & $15.2(2.4)$ & $15.0(2.4)$ & $15.0(2.2)$ & $15.3(2.1)$ & 0.028 \\
\hline $\begin{array}{l}\text { Vegetable protein intake } \\
\text { (g/day) }\end{array}$ & $32.3(11.7)$ & $33.6(11.0)$ & $33.0(11.0)$ & $35.7(10.9)$ & $37.9(10.7)$ & $<0.001$ \\
\hline Animal protein intake (g/day) & $44.3(15.2)$ & $44.0(15.3)$ & $40.9(13.1)$ & $37.5(11.7)$ & $37.3(11.5)$ & $<0.001$ \\
\hline Carbohydrate intake (g/day) & $228(86)$ & $224(74)$ & $218(67)$ & $220(65)$ & $223(54)$ & 0.367 \\
\hline Carbohydrate intake (E\%) & $42.6(6.5)$ & $42.9(5.9)$ & $43.4(6.0)$ & $44.4(5.5)$ & $45.1(5.1)$ & $<0.001$ \\
\hline $\begin{array}{l}\text { Mono-and disaccharides } \\
\text { (g/day) }\end{array}$ & $97(43)$ & $96(38)$ & $98(35)$ & $96(29)$ & $100(26)$ & 0.649 \\
\hline Fibre (g/day) & $21.0(7.5)$ & $22.7(7.0)$ & $23.4(6.5)$ & $25.8(7.1)$ & $27.8(6.9)$ & $<0.001$ \\
\hline Total fat intake (g/day) & $89.4(36.4)$ & $86.5(30.2)$ & $79.4(26.1)$ & $78.6(26.8)$ & $78.0(23.9)$ & $<0.001$ \\
\hline Total fat intake (E\%) & $35.8(5.8)$ & $35.8(5.3)$ & $34.4(5.2)$ & $34.2(5.5)$ & $34.0(5.0)$ & $<0.001$ \\
\hline Saturated fat intake (g/day) & $32.3(13.4)$ & 30.7 (11.9) & $27.4(9.7)$ & $26.6(9.8)$ & $24.6(9.2)$ & $<0.001$ \\
\hline Saturated fat intake (E\%) & $13.0(2.8)$ & $12.7(2.7)$ & $11.8(2.1)$ & $11.5(2.5)$ & $10.7(2.3)$ & $<0.001$ \\
\hline
\end{tabular}

\section{Micronutrient intake per 4.2}

MJ (day)

Calcium (mg)

Folate (mcg)

Potassium (mg)

Riboflavin (mg)

Thiamine (mg)

Vitamin A (RE)

Vitamin B6 $(\mathrm{mcg})$

Vitamin B12 (mcg)

Vitamin C (mg)

$435(149)$
$114(35)$
$1516(280)$
$0.68(0.19)$
$0.49(0.10)$
$604(360)$
$751(17)$
$2.1(1.0)$
$35(16)$

467 (140)

487 (134)

487 (128)

$500(127) \quad<0.001$

128 (39)

139 (44)

$151(43)$

165 (52)

$<0.001$

1594 (286)

1699 (316)

1706 (309)

1753 (293)

$<0.001$

Vitamin E (mg)

$5.8 \quad(1.4)$

$0.72(0.18)$

$0.75(0.20)$

$0.73(0.16)$

$0.74(0.18)$

0.002

$0.50(0.11)$

$0.50(0.10)$

$0.51(0.1)$

0.50 (0.09)

0.052

680 (444)

636 (349)

714 (401)

717 (361)

0.005

764 (16)

800 (17)

820 (17)

853 (17)

$<0.001$

$2.3(1.2)$

$2.2(0.9)$

$2.0(0.8)$

2.1 (0.9)

0.398

40 (21)

49 (23)

51 (21)

$<0.001$

$\mathrm{RE}$, retinol equivalents

* DHD15-index score ranging from zero to 140 points.

${ }^{\dagger}$ Dutch Healthy Diet (index) score based on 8 components without components physical activity and acidic foods and drinks, with a total score ranging from zero (no adherence to Dutch dietary guidelines 2006) to 80 (maximum adherence). ‡ low education=primary school, vocational and lower general secondary education. Moderate=higher secondary education and intermediate vocational training. High=higher vocational education and university. 



\section{Chapter 3}

Using enhanced regression calibration to combine dietary intake estimates from 24-hour recall and food frequency questionnaire improves diet-disease associations

Moniek Looman, Hendriek C. Boshuizen, Edith J.M. Feskens, Anouk Geelen 


\section{Abstract}

Background: Measurement error in dietary intake estimates leads to biased estimates of diet-disease associations. Statistical methods can partly correct the bias when at least one other superior measurement is available. Combining data from 24-hour recalls (24hR) and food frequency questionnaires (FFQ) using enhanced regression calibration (ERC) could result in unbiased association estimates.

Objective: To illustrate the impact of combining $24 \mathrm{hR}$ and FFQ estimates using regression calibration (RC) and ERC on diet-disease associations.

Methods: For 236 subjects of the NQplus study, two $24 \mathrm{hR}$, a FFQ and urinary biomarkers for protein and potassium were collected. Five approaches for obtaining self-reported dietary intake estimates were compared: 1) uncorrected FFQ intakes (FFQ), 2) uncorrected average of two $24 h R(\bar{R}), 3$ ) average of FFQ and $\bar{R}(\bar{F} \bar{R}), 4)$ RC from regressing $24 \mathrm{hR}$ on FFQ, and 5) ERC by adding individual random effects to the RC approach. Empirical attenuation factors (AF) were derived by regressing biomarker measurements on the resulting intake estimates. The AFs were compared using bootstrap (1000 replicates).

Results: Both FFQ and 24hR dietary intake estimates were measured with substantial error and large underestimation was present for protein (FFQ 22.7\%, $\bar{R}$ 14.7\%) and potassium (FFQ 12.5\%, $\bar{R}$ 10.2\%). Using statistical techniques to correct for measurement error (i.e. RC and ERC) reduced bias in dietdisease associations as indicated by their AF approaching 1 (RC 1.14, ERC 0.95 for protein; RC 1.28, ERC 1.34 for potassium). The SD of the corrected intake estimates obtained with ERC was larger, and AF $95 \% \mathrm{Cl}$ intervals were narrower for ERC compared to RC, indicating that using ERC has more power that using RC. However, the difference in AFs between RC and ERC was not statistically significant, indicating no significantly better deattenuation by using ERC compared to RC. AFs larger than 1, observed for the ERC for potassium, indicate possible overcorrection.

Conclusion: Our study highlights the potential of combining FFQ and 24hR data. Using RC and ERC resulted in less biased associations for protein and potassium. In future studies, preferably both FFQ and $24 \mathrm{hR}$ data are collected for the entire study population. 


\section{Introduction}

Despite efforts to develop innovative ways to estimate dietary intake using new emerging technologies, nutrition research and especially large epidemiological studies still rely heavily on traditional dietary assessment tools such as the food frequency questionnaire (FFQ), 24-hour recalls (24hR) and dietary records. These methods all have their strengths and limitations [1]. The FFQ is, for example, relatively cheap and easy to administer, but relies on memory and can lead to social desirable answers, while a limited set of aggregated food items leads to loss of precision, and portion sizes are difficult to assess accurately. The $24 \mathrm{hR}$ and dietary records assess all foods consumed on a single day, but also rely on memory, can lead to social desirable answers and dietary records can influence actual intake due to reactivity. Furthermore, multiple recalls and records are necessary to capture individual habitual intake. Altogether, dietary intake estimates assessed with the FFQ, 24hR or dietary records are known to be biased due to random and systematic measurement error [2, 3].

Measurement error leads to bias, usually attenuation of estimated diet-health associations, loss of precision of estimated associations and loss of power to detect diet-health associations [4]. Statistical methods can partly correct the bias in diet-health associations introduced by measurement errors. To do so, such methods rely on intake estimates from a second (superior) assessment method, i.e. a reference method [1]. The reference method is allowed to have random error, but should be unbiased, that is, free of systematic error. Regression calibration is the most well-known method, in which dietary intake estimates obtained with a reference instrument are regressed on dietary intake estimates obtained with the main method to correct diet-health associations $[5,6]$. Regression calibration is relatively intuitive and simple to use and is applicable in many situations, such as linear and logistic regression and survival analysis.

The $24 \mathrm{hR}$ is often used as reference instrument because objective and unbiased biomarkers of intake are only available for a limited number of nutrients and very costly to collect. Since the development of web-based 24hR and dietary records [7] it is less burdensome for researchers and cheaper to obtain recalls or records from (a subsample of) a study population. Therefore, regression calibration can be used more often to correct for measurement error. With regression calibration equations are obtained that will give predicted dietary intake estimates based on reported intake estimates from the main instrument. However, the calibrated values from the prediction equations only incorporate individual variations that are assessed with the main method, while individual information from the $24 \mathrm{hR}$ measurement is lost. This is unavoidable when $24 \mathrm{hR}$ measurements are only present for a subsample. However, when both the main method (usually FFQ) and the reference instrument (usually 24hR or 
dietary records) are used in the entire study population, this implies unnecessary loss of information. Dietary intake estimates obtained from both methods can be combined to obtain better estimates [8].

The aim of the current study is to demonstrate the impact of combining FFQ and $24 \mathrm{hR}$ estimates by using standard $\mathrm{RC}$ and by using a relative simple extension of $\mathrm{RC}$ using all available information, i.e. enhanced regression calibration (ERC), on resulting diet-disease associations. We will compare protein and potassium intake estimates from RC and ERC with more naive approaches, namely using only the $24 \mathrm{hR}$ as measured, only the FFQ as measured and from averaging $24 \mathrm{hR}$ and FFQ. In this study, the FFQ is used as the main instrument and the average of two telephone administered $24 \mathrm{hR}$ are considered as the superior reference instrument. The extent of the measurement error in the resulting dietdisease associations is assessed for each of the five approaches by calculating the association between the intake estimate and a truly unbiased intake measurement obtained with urinary recovery biomarkers for protein and potassium (i.e. attenuation factors (AF)). With perfect adjustment, this association (or AF) would be 1 .

\section{Methods}

\section{Study population}

For the current analyses, data from the NQplus study collected within the National Dietary Assessment Reference Database (NDARD) was used [9]. Briefly, a total of 2,048 men and women were included between May 2011 and February 2013. They were aged between 20 and 70 years and randomly selected inhabitants of the cities Wageningen, Renkum, Ede, Arnhem, and Veenendaal which are located in the central part of the Netherlands. All participants gave written informed consent before the start of the study. The NQplus study was approved by the medical ethical committee of Wageningen University, and was conducted according to the guidelines of the declaration of Helsinki.

Baseline measurements consisted of, among others, a physical examination, dietary assessment with multiple telephone administered $24 \mathrm{hR}$ and an FFQ, and a 24-hour urine collection. For this study, we selected participants with data of two $24 \mathrm{hR}$, a baseline FFQ and biomarker data of protein and potassium ( $n=236$ ). Twenty-four hour urines were collected in the first year of the study (on average 5 months (interquartile range (IQR) 3-6 months) after the start of the study). The FFQ was administered on average 7.5 months after the start of the study (IQR 5-10 months). The first 24hR was administered on average 7.8 months after the start of the study (IQR 4-16 months); whereas the second recall was administered on average after 15 months (IQR 10-20 months). Recalls of the same participant were at least one month apart. An overview of the timeframe of the different assessments is presented in Figure 3.1. 


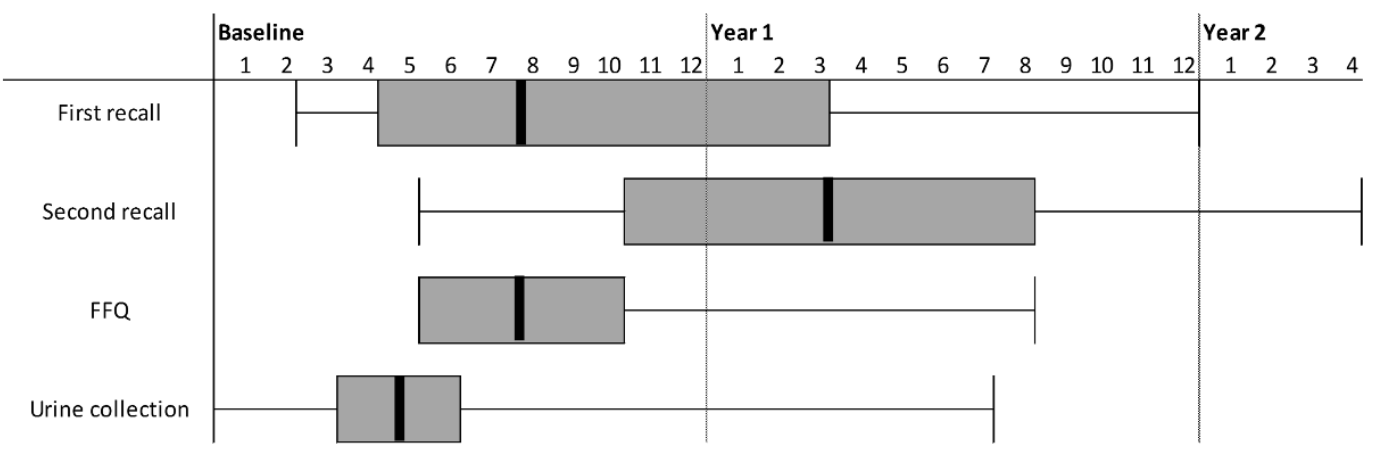

Figure 3.1: Schematic overview of the timeframe of the different dietary assessments and urine collection. The black line represents the median, the grey box represents the interquartile range (p25-p75) and the error bars the minimum and maximum.

\section{Dietary assessment}

$24 h R$

Trained dieticians of the Division of Human Nutrition of Wageningen University made an unannounced phone call to the participant. They asked about foods and drinks consumed the previous day according to a standardized protocol based on the five-step multiple-pass method [10]. Energy and nutrient intakes were estimated using the 2011 Dutch food composition table [11]. For various outcomes (energy, nutrients, and foods) the highest and lowest ten values were checked for errors, such as errors in coding number or amounts (e.g. 150 cups instead of $150 \mathrm{~g}$ of milk).

FFQ

A 180-item semi-quantitative FFQ was self-administered using the open-source online survey tool Limesurvey $^{\mathrm{TM}}$ (LimeSurvey project team/Carsten Schmitz, Hamburg, Germany, 2012). The FFQ has been previously evaluated for energy intake, macronutrients, dietary fibre and selected vitamins [12, 13]. Portion sizes were estimated using natural portions (bread shapes) and commonly used household measures (e.g. spoon and cup). The reference period for reporting was the past month. Average daily nutrient intakes were calculated by multiplying frequency of consumption by portion size and nutrient content per gram using the 2011 Dutch food composition table [11]. 


\section{Biomarker assessment}

Participants received verbal and written instructions for the 24 hour urine collection. The urine collection started after discarding the first voiding on the morning of the collection day and ended after the first voiding on the morning of the next day. To check for completeness of the urine collection, participants were instructed to ingest a tablet containing $80 \mathrm{mg}$ para-amino benzoic acid (PABA) during breakfast, lunch and dinner on the day of the collection. Possible deviations from the protocol (e.g. missing urine) were registered by the participant. The urine collections were mixed, weighted, aliquoted and stored at $-20^{\circ} \mathrm{C}$ until further analysis at the study centre.

The nitrogen content of the urine was assessed with the Kjeldahl technique [14]. The amount of protein was calculated using a nitrogen to protein conversion factor of 6.25 [15] and an average ratio of urinary nitrogen excretion to dietary nitrogen of 0.81 [16] was assumed. Potassium in urine were determined with an ion-selective electrode and potassium intake was calculated taking into account $19 \%$ potassium [17] extra-renal and faecal losses. PABA in urine was assessed by the HPLC method. Incomplete urines, based on the cut-off value of 78\% PABA recovery [18], were excluded from the analysis $(n=16)$.

\section{Combining FFQ and 24hrecalls}

Measurement error model

A diet-disease model is usually structured in the following way:

$$
E(Y \mid T)=\beta_{0}+\beta_{1} T
$$

with disease $\mathrm{Y}$ related to dietary exposure of interest $\mathrm{T}$ through a generalized linear model. $\mathrm{E}$ denotes the expectation of developing disease $\mathrm{Y}$ given consumption of $\mathrm{T}$.

However, as stated previously, dietary exposures are rarely measured without measurement error. Therefore, the true value of T cannot be measured. Instead, we use the following calibration model to express the expected dietary exposure measured with measurement instrument $Q$ :

$$
E(T \mid Q)=\vartheta_{0}+\vartheta_{1} Q
$$

Where $७ 0$ and $७ 1$ represent the systematic errors.

If we replace $T$ with $E(T \mid Q)$ in our diet-disease model we obtain:

$$
E(Y \mid Q)=\beta_{0}+\beta_{1}(E(T \mid Q))=\beta_{0}+\beta_{1}\left(\vartheta_{0}+\vartheta_{1} Q\right)
$$


To be able to detect the association between the dietary exposure of interest and the disease, the expected value of T needs to be as close to the true intake as possible. Otherwise, the added error from measurement error will reduce the correlation between $Y$ and $Q$, and thus the power to detect an association.

\section{Approaches to combine FFQ and $24 \mathrm{hR}$}

In this study, we used the FFQ as main instrument and the average of two $24 \mathrm{hR}$ as reference instrument. We assumed that the $24 \mathrm{hR}$ provides unbiased estimates of usual intake and contains only random within-person error. We present five approaches to obtain intake estimates for use in dietdisease associations (Table 3.1). First, we used the uncalibrated FFQ estimates (FFQ). For the second approach the mean of two $24 \mathrm{hR}(\overline{\mathrm{R}})$ is used. The third approach is simply the average of the FFQ and $\overline{\mathrm{R}}$ $(\bar{F} \bar{R})$. Fourth is a regression calibration $(R C)$ of regression the average value of both $24 \mathrm{hR}$ measurements per person on $F F Q$, resulting in $E(R \mid Q)$. When $R$ is unbiased, this is equal to $E(T \mid Q)$. The fifth and last approach is the enhanced regression calibration (ERC). ERC is an extension of RC in which the individual random effect is included in the regression calibration equation [19]. While RC can be used if intake data from a second method are available for a subsample of the population, ERC needs data from two methods for all subjects in a population. The following formula is used for the ERC:

$$
E(T \mid R 1, R 2, Q)=w \times \bar{R}+(1-w) \times E(T \mid Q)
$$

Where $\bar{R}$ is the average of two $24 h R, E(T \mid Q)$ is set equal to $E(R \mid Q)$, assuming that $R$ is unbiased, and w is $\operatorname{var}(u) /(\operatorname{var}(u)+\operatorname{var}(e) / 2)$, where $\operatorname{var}(u)$ is between person-variance in $24 \mathrm{hR}$ and $\operatorname{var}(\mathrm{e})$ is withinperson variance in $24 \mathrm{hR}$.

Proc Reg was used to obtain RC estimates and Proc Mixed was used to obtain estimates for ERC. The SAS syntax is given in Supplemental file 3.1.

Table 3.1: Overview of the five approaches used in the current study

\begin{tabular}{ll}
\hline 1$) \mathrm{FFQ}$ & Uncorrected FFQ estimate \\
2) $\bar{R}$ & Uncorrected average of two 24hR estimates \\
3) $F \bar{R}$ & Average of 1 ) uncorrected FFQ estimate and 2) uncorrected average of two 24hR estimates \\
4) $R C$ & Predicted intake estimate based on regression of the average of two 24hR estimates on FFQ \\
5) $\mathrm{ERC}$ & Predicted intake estimate based on a mixed model predicting 24hR from FFQ, including the individual \\
& random effect estimate \\
\hline
\end{tabular}




\section{Statistical analyses}

Descriptive statistics were presented in percentages and as means with their standard deviation. The percentage bias was calculated by dividing the difference between the intake assessed by one of the approaches and the intake as estimated from the biomarker, divided by the intake from the biomarker. A linear regression model was used to calculate empirical AFs with the biomarker regressed on the intake estimate obtained by each approach. The attenuation factor provides information on the extent to which diet-disease associations are affected by measurement error. Regressing a recovery biomarker on a perfect intake estimate, that is, an estimate that should deliver an unbiased estimate of association, should result in a regression coefficient (i.e. AF) of 1. An AF lower than 1 indicates attenuation of the diet-disease association due to measurement error, with a larger deviation from 1 indicating more attenuation. To test whether empirical AFs of the different approaches differed statistically significantly from each other, we used a bootstrap approach (1000 replicates). To correct for multiple testing, we used the Bonferroni correction and thus considered a $p$-value $<0.01$ statistically significant for the comparison of the AFs between the five approaches. Finally, we used the empirical AFs to illustrate the impact of measurement error in estimates from the five approaches using an example diet-disease association with an assumed true relative risk (RR) of 2.0. The observed RR for each approach was calculated with the following formula: $R R_{\text {true }}=\left(R R_{\text {observed }}\right)^{1 / A F}$. Rewriting the formula gives: $R_{\text {observed }}=\left(R_{\text {true }}\right)^{A F}$. All statistical tests were performed using SAS 9.3 (SAS institute Inc. Cary, NC, USA).

\section{Results}

At baseline, participants ( $n=236 ; 89$ men and 147 women) were on average 54.0 (SD 10.9) years old, had a mean body mass index of 25.4 (SD 4.0 ) $\mathrm{kg} / \mathrm{m}^{2}$ and $68.5 \%$ was classified as highly educated (university or college degree).

The mean intakes estimated using the RC and ERC approaches were similar to $\overline{\mathrm{R}}$, as this is the reference method used. (Table 3.2). The SD of the ERC was larger compared to that of RC due to the inclusion of the random effect in the estimation, thus theoretically increasing power to detect an association with disease or other outcome. However, the SD was still considerably smaller than the SD of the $\bar{R}$ and biomarker, which are high due to random day-to-day error.

Both the $F F Q$ and $\bar{R}$ underestimated protein and potassium intake compared to their respective urinary biomarkers (Table 3.2), with FFQ showing the largest underestimation. Protein intake was underestimated by $22.7 \%$ when using FFQ estimates and $14.7 \%$ when using $\bar{R}$ estimates as compared 
to protein intake based on urinary nitrogen. Averaging FFQ and $24 \mathrm{hR}(\overline{\mathrm{F}} \overline{\mathrm{R}})$ resulted in an average underestimation of $18.7 \%$, whereas estimates based on RC underestimated $13.8 \%$ and ERC estimates underestimated protein intake with $14.1 \%$. For potassium, the FFQ underestimated potassium intake the most, with an average bias of $12.5 \%$. For the $\bar{R}$ the underestimation was $10.2 \%$ on average, whereas for $\bar{F} \bar{R}$ this was $11.3 \%$. RC and ERC underestimated potassium intake the least with $8.4 \%$ underestimation for RC and 9.2\% for ERC.

Table 3.2: Mean estimated intake and bias per approach.

\begin{tabular}{|c|c|c|c|c|}
\hline & \multicolumn{2}{|c|}{ Protein (g) } & \multicolumn{2}{|c|}{ Potassium (mg) } \\
\hline & Mean (SD) & $\operatorname{Bias}(\%)^{a}$ & Mean (SD) & $\operatorname{Bias}(\%)^{a}$ \\
\hline Biomarker & $97.2(22.1)$ & & 4047 (1331) & \\
\hline 1) FFQ & $73.1(16.6)$ & $-22.7(18.7)$ & 3288 (751) & $-12.5(32.6)$ \\
\hline 2) $\bar{R}$ & $80.3(21.1)$ & $-14.7(24.2)$ & 3400 (824) & $-10.2(29.9)$ \\
\hline 3) $\bar{F} \bar{R}$ & 76.7 (15.7) & $-18.7(18.2)$ & 3344 (683) & $-11.3(29.2)$ \\
\hline 5) $\mathrm{RC}$ & $80.3(8.0)$ & $-13.8(18.1)$ & 3400 (413) & $-8.4(30.8)$ \\
\hline 6) ERC & $80.3(10.9)$ & $-14.1(18.1)$ & $3400(523)$ & $-9.2(28.8)$ \\
\hline
\end{tabular}

a percentage (\%) bias was calculated on the individual level using the biomarker as the true intake and displayed as mean (SD).

For protein intake estimates, the empirical AF was smallest for $\overline{\mathrm{R}}$ estimates $(0.40)$ and slightly but not significantly higher for FFQ intake estimates (0.55) (Figure 3.2a). The average of FFQ and $\bar{R}(\bar{F} \bar{R})$ improved intake estimates with an AF of 0.66 and performed significantly better than $F F Q$ and $\bar{R}$ intake estimates. The RC and ERC intake estimates produced significantly higher AFs being 1.14 and 0.95 , respectively. For potassium, AFs were smallest for FFQ estimates ( 0.70$)$ and slightly but not significantly higher for $\bar{R}$ intake estimates (0.80) (Figure 3.2b). The AF was 1.01 for the $\bar{F} \bar{R}$ estimates, whereas the AFs for RC and ERC were higher than 1 (1.28 and 1.34 respectively). The latter value differs statistically significantly from 1, indicating that a correction using ERC based on the average of two $24 \mathrm{hr}$ recalls as reference value could lead to an overestimation of the strength of the association between potassium intake and health effect. For protein, however, an ERC correction using the average of two $24 \mathrm{hr}$ recalls from our data seems to yield an approximately correct strength of association.

While for both nutrients RC and ERC AFs did not differ statistically significantly from each other, the ERC estimate had the smallest $95 \% \mathrm{Cl}$, indicating higher precision of the $\mathrm{AF}$ and potentially more power to detect diet-disease association.

To illustrate the impact of applying RC or ERC on dietary intake estimates used in diet-disease associations, we give an example using AFs obtained for the five approaches for protein and potassium. We assume to have a hypothetical diet-disease association with a true relative risk (RR) of 2.0. If we would use the FFQ estimate for protein, we would obtain an observed RR of 1.46 (i.e. $2.0^{0.55}$ ), whereas 
with the $\bar{R}$ estimate a RR of 1.36 (i.e. $2.0^{0.40}$ ) would be obtained (Figure 3.3, left side). For the $\bar{F} \bar{R}$ an $R R$ of 1.58 (i.e. $2.0^{0.66}$ ) would be observed. Using RC intake estimates for protein would give a RR of 2.20 (i.e. $2.0^{1.14}$ ). For the ERC the observed RR would be 1.93 (i.e. $2.0^{0.95}$ ) which is closest to the true RR. For potassium, the observed relative risk would be 1.62 (i.e. $2.0^{0.70}$ ) when using FFQ intake estimates and 1.74 (i.e. $2.0^{0.80}$ ) when using $\bar{R}$ intake estimates (Figure 3.3, right side). Using $\bar{F} \bar{R}$ intake estimates would result in an observed RR of 2.01 (i.e. 2.0 $0^{1.01}$ ), which is closest to the true RR. Using RC and ERC would lead to an observed RR of 2.45 (i.e. $2.0^{1.28}$ ) and 2.53 (i.e. $2.0^{1.34}$ ), respectively, indicating overcorrection as these are higher than the true RR of 2.0.
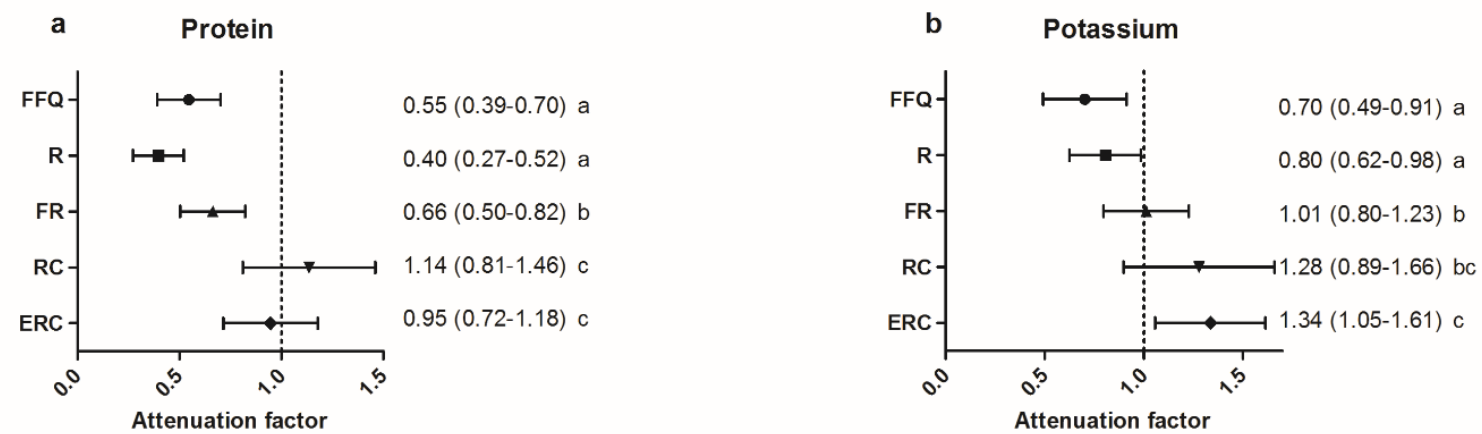

Figure 3.2: Empirical attenuation factors $(95 \% \mathrm{Cl})$ for the 5 approaches for a) protein and b) potassium from regression of the biomarker on the intake estimates. Different letters indicate statistically significant attenuation factors.

$R=$ mean of 2 telephone-based 24 hour recalls, $F R=$ mean of $F F Q$ and 2 telephone-based 24 hour recalls, $R C=$ regression calibration with the $\mathrm{FFQ}$ as main instrument and 2 telephone-based 24 hour recalls as superior instrument, and $E R C=$ enhanced regression calibration with the FFQ as main instrument and 2 telephonebased 24 hour recalls as superior instrument.

\section{Discussion}

RC and ERC significantly improved estimates of diet-disease associations, as demonstrated by AFs approaching 1 , as compared to simply averaging estimates from two different assessment methods and uncorrected estimates. For both potassium and protein, RC and ERC AFs did not differ statistically significantly from each other. The ERC AF, however, had the narrowest $95 \% \mathrm{Cl}$, indicating higher precision of the $\mathrm{AF}$ and potentially more power to detect diet-disease associations than the $\mathrm{RC}$ approach. 


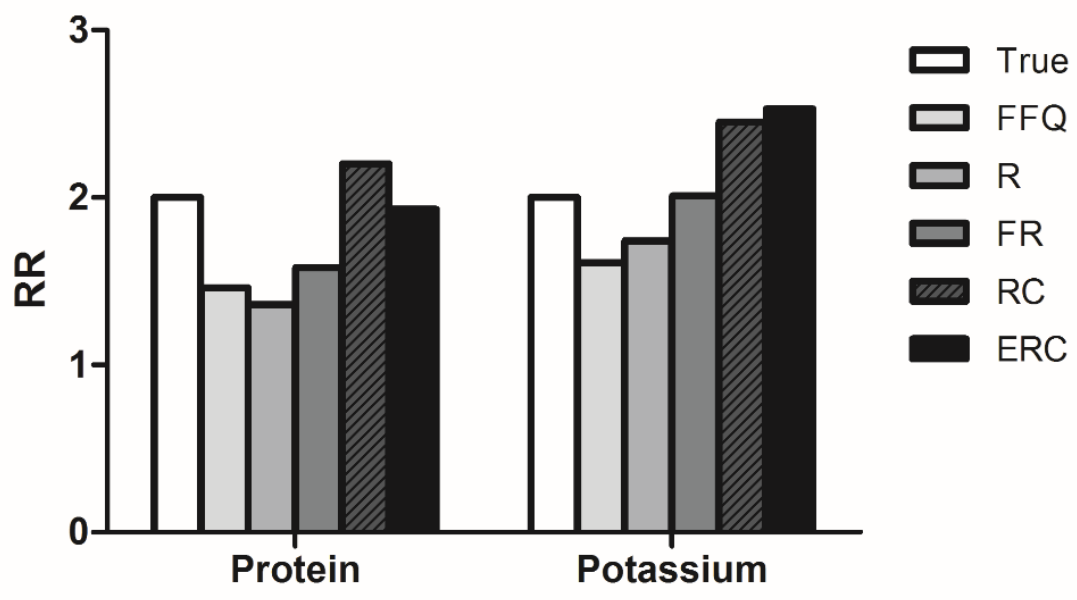

Figure 3.3: Visualization of the impact of the five presented approaches on diet-disease relative risks assuming a hypothetical true relative risk of 2.0 .

$\mathrm{R}=$ mean of 2 telephone-based 24 hour recalls, $F R=$ mean of FFQ and 2 telephone-based 24 hour recalls, $R C=$ regression calibration with the FFQ as main instrument and 2 telephone-based 24 hour recalls as superior instrument, and ERC = enhanced regression calibration with the FFQ as main instrument and 2 telephonebased 24 hour recalls as superior instrument.

Although our finding of substantial measurement error is not new $[2,20]$, it does underscore the importance of validation studies. In our study, we used the average of two $24 \mathrm{hR}$ as reference instrument for the regression calibration and enhanced regression calibration approaches, as is common in nutritional epidemiology. However, this requires the assumption that $24 \mathrm{hR}$ intakes are unbiased, which often does not hold [21]. Also in our study, we can see that this assumption is violated, as there is an average underreporting of $14.7 \%$ for protein and $10.2 \%$ for potassium intake estimates based on two $24 \mathrm{hR}$ compared to intake estimates based on urinary recovery biomarkers. It should be noted that RC and ERC calibrate the main instrument (i.e. the FFQ) to the reference instrument (i.e. $24 \mathrm{hR}$ ) to correct the intake and distribution on a population level. Estimates obtained with RC and ERC do not reflect corrected individual intake levels and cannot be used as such.

However, the aim of our study was not to corrected individual intake levels, but to assess the impact of combining FFQ and $24 \mathrm{hR}$ estimates on resulting diet-disease associations, as this is usually the main interest in nutritional epidemiology. In our study, we used AF as measure for bias in resulting dietdisease associations, with an AF of 1 indicating no bias present. AFs for the FFQ and 24hR showed that there was substantial bias, especially for protein intakes, that would result in attenuation of dietdisease associations (i.e. AF $<1$ ). Averaging FFQ and $24 \mathrm{hR}$ resulted in AFs closer to 1 , while using RC and ERC improved protein estimates with resulting AFs close to 1 . However, for potassium, using RC and ERC led to overcorrection, as indicated by AFs $>1$. This possible overcorrection warrants further investigation. 
The mean intake estimates and the AFs for RC and ERC did not differ much, which could raise the question if ERC has additional benefits to RC, as it implies a lot of extra effort and labour to collect two $24 \mathrm{hR}$ for each participant instead of only a subsample. However, the SD of RC is smaller than the SD of the ERC, indicating a narrower intake distribution resulting from RC than from ERC. This narrower distribution of the RC makes it more difficult to discriminate between individuals and indicates a loss of power. The wider distribution of the ERC intake estimates thus underlines the theoretical advantage of the ERC having more power to detect diet-disease associations than RC. Another example can be found in the $95 \% \mathrm{Cl}$ of the AFs being smaller for ERC than for RC indicating more precision with the ERC in estimating diet-disease associations.

In the current study, we used the average of two telephone administered $24 \mathrm{hR}$ as reference instrument, as this is a well-documented reference instrument. However, this method is too labour intensive and expensive to administer in the entire study population of large epidemiological studies. Using recalls as reference method would thus be limited to validation studies, excluding the possibility to use the proposed ERC method. However, with the availability of $24 \mathrm{hR}$ administered via the internet (e.g. ASA24 [22], Compl-eat [23]) costs of collecting $24 \mathrm{hR}$ are substantially reduced making the webbased $24 \mathrm{hR}$ a viable option for large epidemiological studies. Evaluations indicated that web-based $24 \mathrm{hR}$ are in general in good agreement with interview-administered 24hR [7]. For example, the ASA24 had an average relative mean difference of $1.6 \%$ for energy intake, $2.9-11.1 \%$ for macronutrients and $-4.2-11.9 \%$ for micronutrients, compared to the telephone administered 24hR [24]. Furthermore, the Dutch web-based $24 \mathrm{hR}$ tool Compl-eat underestimated macronutrients on average with $8 \%$ and micronutrients with $13 \%$ compared to telephone administered $24 \mathrm{hR}$ [23]. These results indicate that web-based $24 \mathrm{hR}$ could also be used as reference instruments for the RC and ERC.

We propose a rather simple method to combine the $24 \mathrm{hR}$ and FFQ dietary intake estimates. Others have shown that adding covariates such as BMI to the regression calibration model might improve the regression calibration $[25,26]$. However, these covariates should be measured accurately and without bias to prevent introducing additional bias. Furthermore, covariates could also act as mediator or confounder in the diet-disease association of interest, and including such covariates in the model could potentially even lead to bias in the regression calibration rather than improvement $[4,27]$. Another method suggested including biomarker measures in the regression calibration model to provide unbiased diet-disease estimates [28]. The advantage of using biomarkers is that they are objectively measured and are assumed to have uncorrelated errors with the self-report instrument, in contrast to using two self-report dietary assessment tools such as FFQ and 24hR. Additionally, recovery biomarkers are free of intake-related bias. A limitation is the limited availability of biomarkers for nutrient intake, and the substantial burden and costs associated with biomarker measurements. 
Therefore, combining two self-report dietary assessment tools has much more potential in the field of nutritional epidemiology.

A limitation of the current study is that recovery biomarkers were needed for validation of the proposed approaches. Therefore, we were limited to studying protein and potassium intake estimates as no other recovery biomarkers were available. We can only speculate whether the proposed approaches also improve estimates for intakes of other (micro) nutrients, energy and foods. However, the study of Carroll et al. demonstrated a large gain in power and precision when combining FFQ and two $24 \mathrm{hR}$ data for micronutrients and food groups compared to only two $24 \mathrm{hR}$ or one FFQ [8]. They used the $\mathrm{NCl}$ method to obtain usual intake estimates $[29,30]$ and only used the frequency information from the FFQ as covariate in the regression calibration [31], whereas in the ERC more information from the FFQ is used to correct intake estimates and consequently diet-disease associations. The benefits of combining FFQ and $24 \mathrm{hR}$ demonstrated by Carroll et al. should also apply to the ERC approach in our study, suggesting the suitability of the ERC for micronutrients and food groups. As the $24 \mathrm{hR}$ tends to be less reliable in estimation of episodically consumed foods, we believe the largest gain in improving intake estimates for diet-disease associations can be achieved in those foods.

Future research should focus on identifying error structures of intake estimates from using the RC and ERC approach using measurement error models. This information can be used to further improve the regression calibration models, possibly by including other covariates. However, keeping the simplicity of the suggested ERC approach is desirable.

\section{Conclusions}

Measurement error is a serious problem in nutrition research. Our study highlights the potential of combining $\mathrm{FFQ}$ and $24 \mathrm{hR}$ data using $\mathrm{RC}$ and ERC, simple approaches, with substantial impact on correcting diet-disease associations. The availability of web-based $24 \mathrm{hR}$ reduces burden and costs, making it easier to use in large population study. Preferably, FFQ and 24hR data are collected for the entire study population and ERC is used. 
Chapter 3

Acknowledgements: We thank all participants contributing to the NQplus study for their time and contributions, and the research staff and students for collecting the data of the NQplus study.

Compliance with ethical standards: The NQplus study was approved by the medical ethical committee of Wageningen University, and was conducted according to the guidelines of the declaration of Helsinki. All participants gave written informed consent before the start of the study.

Conflicts of interest: The authors declare that they have no competing interests.

Funding: The NQplus study was funded by Wagenigen University and ZonMW (grant number 911100030), The Netherlands Organization for Health Research and Development, The Hague. 


\section{References}

1. Willett WC, Nutritional Epidemiology. 3rd ed. 2013, New York: Oxford University Press.

2. Kipnis, V., A.F. Subar, D. Midthune, et al., Structure of dietary measurement error: results of the OPEN biomarker study. Am. J. Epidemiol., 2003. 158(1): p. 14-21; discussion 22-6.

3. Prentice, R.L., Y. Mossavar-Rahmani, Y. Huang, et al., Evaluation and comparison of food records, recalls, and frequencies for energy and protein assessment by using recovery biomarkers. Am. J. Epidemiol., 2011. 174(5): p. 591-603.

4. $\quad$ Freedman, L.S., A. Schatzkin, D. Midthune, et al., Dealing with dietary measurement error in nutritional cohort studies. J. Natl. Cancer Inst., 2011. 103(14): p. 1086-92.

5. Rosner, B., W.C. Willett, and D. Spiegelman, Correction of Logistic-Regression Relative Risk Estimates and Confidence-Intervals for Systematic within-Person Measurement Error. Stat. Med., 1989. 8(9): p. 1051-1069.

6. Spiegelman, D., A. McDermott, and B. Rosner, Regression calibration method for correcting measurement-error bias in nutritional epidemiology. Am. J. Clin. Nutr., 1997. 65(4): p. 1179-1186.

7. Timon, C.M., R. van den Barg, R.J. Blain, et al., A review of the design and validation of web-and computer-based 24- $h$ dietary recall tools. Nutr. Res. Rev., 2016. 29(2): p. 268-280.

8. Carroll, R.J., D. Midthune, A.F. Subar, et al., Taking advantage of the strengths of 2 different dietary assessment instruments to improve intake estimates for nutritional epidemiology. Am. J. Epidemiol., 2012. 175(4): p. 340-7.

9. Brouwer-Brolsma, E.M., M.T. Streppel, L. van Lee, et al., A National Dietary Assessment Reference Database (NDARD) for the Dutch Population: Rationale behind the Design. Nutrients, 2017. 9(10).

10. Conway, J.M., L.A. Ingwersen, B.T. Vinyard, et al., Effectiveness of the US Department of Agriculture 5-step multiplepass method in assessing food intake in obese and nonobese women. Am. J. Clin. Nutr., 2003. 77(5): p. 1171-8.

11. NEVO-tabel, Dutch Food Composition Table 2011/version 3. 2011, National Institute for Publich Health and the Environment/Netherlands Nutrition Centre.

12. Siebelink, E., A. Geelen, and J.H. de Vries, Self-reported energy intake by FFQ compared with actual energy intake to maintain body weight in 516 adults. Br. J. Nutr., 2011. 106(02): p. 274-281.

13. Verkleij-Hagoort, A.C., J.H. de Vries, M.P. Stegers, et al., Validation of the assessment of folate and vitamin B12 intake in women of reproductive age: the method of triads. Eur. J. Clin. Nutr., 2007. 61(5): p. 610-615.

14. Hambleton, L.G. and R.J. Noel, Protein Analysis of Feeds, Using a Block Digestor. J Assoc Off Ana Chem, 1975. 58(1): p. 143-145.

15. Jones, D., Factors for converting percentages of nitrogen in foods and feeds into percentages of proteins, U.D.o. Agriculture, Editor. 1941, US Department of Agriculture: Washington DC.

16. Bingham, S.A. and J.H. Cummings, Urine Nitrogen as an Independent Validatory Measure of Dietary-Intake - a Study of Nitrogen-Balance in Individuals Consuming Their Normal Diet. Am. J. Clin. Nutr., 1985. 42(6): p. 1276-1289.

17. Freisling, H., M.M.E. van Bakel, C. Biessy, et al., Dietary reporting errors on $24 \mathrm{~h}$ recalls and dietary questionnaires are associated with BMI across six European countries as evaluated with recovery biomarkers for protein and potassium intake. Br. J. Nutr., 2012. 107(6): p. 910-920.

18. Jakobsen, J., L. Ovesen, S. Fagt, et al., para-aminobenzoic acid used as a marker for completeness of 24 hour urine: Assessment of control limits for a specific HPLC method. Eur. J. Clin. Nutr., 1997. 51(8): p. 514-519.

19. Midthune, D. Combining self-report dietary assessment instruments to reduce the effects of measurement error. Measurement Error Webinar Series 2011 [cited 2017 October 30th].

20. Geelen, A., O.W. Souverein, M.C. Busstra, et al., Comparison of approaches to correct intake-health associations for FFQ measurement error using a duplicate recovery biomarker and a duplicate $24 \mathrm{~h}$ dietary recall as reference method. Public Health Nutr., 2015. 18(2): p. 226-33.

21. Freedman, L.S., J.M. Commins, W. Willett, et al., Evaluation of the 24-Hour Recall as a Reference Instrument for Calibrating Other Self-Report Instruments in Nutritional Cohort Studies: Evidence From the Validation Studies Pooling Project. Am. J. Epidemiol., 2017. 186(1): p. 73-82.

22. Subar, A.F., S.I. Kirkpatrick, B. Mittl, et al., The Automated Self-Administered 24-hour dietary recall (ASA24): a resource for researchers, clinicians, and educators from the National Cancer Institute. J. Acad. Nutr. Diet., 2012. 112(8): p. 1134-7.

23. Meijboom, S., M. Van Houts-Streppel, C. Perenboom, et al., Evaluation of dietary intake assessed by the Dutch selfadministered web-based dietary 24-h recall tool (Compl-eat ${ }^{T M}$ ) against interviewer-administered telephone-based 24- $h$ recalls. Journal of nutritional science, 2017. 6.

24. Thompson, F.E., S. Dixit-Joshi, N. Potischman, et al., Comparison of Interviewer-Administered and Automated SelfAdministered 24-Hour Dietary Recalls in 3 Diverse Integrated Health Systems. Am. J. Epidemiol., 2015. 181(12): p. 970-8.

25. Prentice, R.L., M. Pettinger, L.F. Tinker, et al., Regression calibration in nutritional epidemiology: example of fat density and total energy in relationship to postmenopausal breast cancer. Am. J. Epidemiol., 2013. 178(11): p. 166372.

26. Prentice, R.L. and Y. Huang, Measurement error modeling and nutritional epidemiology association analyses. Canadian Journal of Statistics-Revue Canadienne De Statistique, 2011. 39(3): p. 498-509. 
27. Agogo, G.O., H. van der Voet, P. van't Veer, et al., Use of two-part regression calibration model to correct for measurement error in episodically consumed foods in a single-replicate study design: EPIC case study. PLoS One, 2014. 9(11): p. e113160.

28. Freedman, L.S., D. Midthune, R.J. Carroll, et al., Using regression calibration equations that combine self-reported intake and biomarker measures to obtain unbiased estimates and more powerful tests of dietary associations. Am. J. Epidemiol., 2011. 174(11): p. 1238-45.

29. Freedman, L.S., P.M. Guenther, S.M. Krebs-Smith, et al., A population's distribution of Healthy Eating Index-2005 component scores can be estimated when more than one 24-hour recall is available. J. Nutr., 2010. 140(8): p. 152934.

30. Freedman, L.S., P.M. Guenther, K.W. Dodd, et al., The population distribution of ratios of usual intakes of dietary components that are consumed every day can be estimated from repeated 24-hour recalls. J. Nutr., 2010. 140(1): p. 111-6.

31. Kipnis, V., D. Midthune, D.W. Buckman, et al., Modeling data with excess zeros and measurement error: application to evaluating relationships between episodically consumed foods and health outcomes. Biometrics, 2009. 65(4): p. 1003-10. 


\section{Supplementary material}

Supplemental file 3.1: The SAS syntax for the Proc Reg procedure to obtain regression calibration (RC) estimates and the SAS syntax for the Proc Mixed procedure to obtain enhanced regression calibration (ERC) estimates.

a. SAS syntax for regression calibration of the FFQ using two $24 \mathrm{hR}$ as reference instrument

For the regression procedure, the mean of the two $24 \mathrm{hR}(\overline{\mathrm{R}})$ needs to be calculated and is the dependent variable in the regression equation. The FFQ estimate is the independent variable in the equation. Generic labels for the variables are used (recalls is named $\bar{R}, F F Q$ is named FFQ). The dataset is called mydataset.

Proc reg data=mydataset;

Model $\bar{R}=F F Q$;

Run;

Parameter estimates can be found in the output. Regression calibration equation to obtain RC predicted dietary intake estimates:

$\mathrm{RC}=$ intercept parameter estimate $+(\mathrm{FFQ}$ parameter estimate*FFQ)

b. SAS syntax for enhanced regression calibration of the FFQ using two $24 \mathrm{hR}$ as reference instrument while adding random effects to the regression calibration

For the mixed procedure, the dataset needs to be ordered using a long data format, i.e. $24 \mathrm{hR} 1$ en $24 \mathrm{hR} 2$ are listed below each other for each participant. FFQ estimates are duplicated on each row. Generic labels for the variables are used (participant identifier is named pID, recalls is named $24 \mathrm{hR}, \mathrm{FFQ}$ is named FFQ). The dataset is called mydataset.

Ods output solutionr=solutionrandom solutionf=solutionfixed;

Proc mixed data=mydataset;

Class pID;

Model $24 \mathrm{hR}=\mathrm{FFQ} /$ solution;

Random intercept /subject=pID solution;

Run;

Parameter estimates can be found in the output. Regression calibration equation to obtain ERC predicted dietary intake estimates:

$\mathrm{ERC}=$ estimateSF1 + (estimateSF2*FFQ) + estimateR

Where estimateSF1 represents the fixed effect intercept estimate, estimateSF2 represents the fixed effect FFQ parameter estimate, both obtained from the solutionfixed output. EstimateR represents the random effect individual estimate, obtained from the solutionrandom output. 



\section{Chapter 4}

Adverse pregnancy outcomes in treated gestational diabetes and untreated borderline gestational diabetes

Moniek Looman, Anouk Geelen, Dorien Zandstra, Rahul A.K. Samlal, Rik Heijligenberg, Edith J.M. Feskens 


\section{Abstract}

Objective: The first objective is to compare risk of adverse pregnancy outcomes between women with treated gestational diabetes (GDM), women with untreated borderline gestational diabetes (BGDM) and women with normal glucose tolerance (NGT). Secondly, we want to verify if macrosomia is associated with adverse pregnancy outcomes and whether macrosomia is a mediator in the association between glucose tolerance during pregnancy (i.e. GDM, BGDM, NGT) and adverse pregnancy outcomes.

Methods: A retrospective analysis was performed using medical file data of 1049 women with a singleton pregnancy who underwent glucose screening during pregnancy and who delivered in hospital Gelderse Vallei between 1-1-2010 and 31-12-2014. Prevalence ratios (PR) and 95\% confidence intervals $(95 \% \mathrm{Cl})$, adjusted for gestational age, maternal age and parity, were estimated.

Results: High rates of macrosomia were observed in women with treated GDM (17\%) and untreated BGDM (25\%). Compared to women with NGT, women with untreated BGDM had a higher risk of unscheduled Caesarean section ( $\mathrm{PR}=1.90,95 \% \mathrm{Cl}$ 1.20-2.99), total Caesarean section ( $\mathrm{PR}=1.51,95 \% \mathrm{Cl}$ 1.14-2.00) and laceration ( $P R=1.24,95 \% \mathrm{Cl} 1.06-1.46)$. Women with treated GDM had a higher risk of laceration compared to women with NGT ( $\mathrm{PR}=1.28,95 \% \mathrm{Cl} 1.14-1.45)$. Macrosomia did not mediate associations between glucose tolerance and adverse pregnancy outcomes, as adding macrosomia to the model did not change prevalence ratios.

Conclusions: Macrosomia rates were high in both treated GDM and untreated BGDM. Women with untreated BGDM had a higher risk of (unscheduled) Caesarean section and laceration than women with NGT, whereas for women with treated GDM risk of laceration, but not other outcomes, was higher. 


\section{Introduction}

Incidence of gestational diabetes mellitus (GDM) is increasing worldwide [1, 2]. In 2008, the Hyperglycaemia and Adverse Pregnancy Outcomes (HAPO) study demonstrated that maternal glucose levels during pregnancy are associated with several adverse pregnancy outcomes including high birthweight, premature delivery, delivery by Caesarean section, neonatal hypoglycaemia, shoulder dystocia, birth injury, and pre-eclampsia [3]. Treatment of GDM with tight glucose monitoring, dietary counselling and, if necessary, insulin can reduce the risk of these adverse pregnancy outcomes [4-6]. However, it is not clear if the risk of adverse pregnancy outcomes in women with treated GDM is comparable to the risk of women with normal glucose tolerance (NGT) or whether treated GDM still increases risk of adverse pregnancy outcomes.

The association between maternal glucose levels and adverse pregnancy outcomes in the HAPO study was linear. This implies that women with abnormal glucose values below the GDM diagnostic criteria, i.e. borderline gestational diabetes mellitus (BGDM), also have a higher risk of adverse pregnancy outcomes compared to women with NGT. An Australian study showed that BGDM affects approximately $6-8 \%$ of the pregnant women [7]. However, little is known about the risk of adverse pregnancy outcomes for these women. A Turkish study found no increased risk of adverse pregnancy outcomes for women with BGDM compared to women with normal glucose tolerance [8], whereas three Australian studies did find higher risk of several adverse health outcomes for mothers with BGDM and their children $[7,9,10]$. In the Netherlands, women with BGDM do not receive treatment and it is not known if women with untreated BGDM are at increased risk of adverse pregnancy outcomes compared to women with NGT.

Results of the HAPO study clearly demonstrated that GDM increases risk of macrosomia (i.e. birthweight $>4000 \mathrm{~g}$ ). Giving birth to a macrosomic infant is associated with higher risk of adverse pregnancy outcomes, including neonatal death, shoulder dystocia, pre-eclampsia, instrumental delivery, and unplanned Caesarean section [11]. Several of these adverse pregnancy outcomes are also associated with GDM and we hypothesize that macrosomia is a mediator in the association between hyperglycaemia and adverse pregnancy outcomes.

The first objective of the current study is to compare risk of adverse pregnancy outcomes between women with treated GDM, women with untreated BGDM and women with NGT. Secondly, we want to verify if macrosomia is associated with adverse pregnancy outcomes in our Dutch population and whether macrosomia is a mediator in the association between glucose tolerance during pregnancy (i.e. GDM, BGDM, NGT) and adverse pregnancy outcomes. 


\section{Materials and Methods}

\section{Study design and study population}

Electronic medical record information from Hospital Gelderse Vallei Ede in the Netherlands was used for this retrospective analysis. There were 8535 birth records of hospital Gelderse Vallei available for the study period 1-1-2010 to 31-12-2014. Of the women with multiple pregnancies during the study period, the first pregnancy of these women was included for analysis and further pregnancies were omitted ( $n=2846$ ). Subsequently, women were divided into three mutually exclusive groups based on glucose tolerance during pregnancy. We identified women diagnosed with GDM ( $n=365)$, women with BGDM ( $n=219)$ and women with normal glucose tolerance (NGT) $(n=4923)$. For the NGT group, all records of women with NGT who gave birth to a macrosomic infant $(n=691)$ and a representative sample of the remaining women in the NGT group $(n=1000)$ were selected for data extraction. We included all records of women who gave birth to a macrosomic infant to ensure enough cases for the second objective of this study, i.e. assess whether macrosomia is a mediator in the association of GDM with adverse pregnancy outcomes. Women who did not undergo glucose screening $(n=1088)$, women with missing birth information ( $n=112)$, women with a multifetal pregnancy $(n=26)$ and women with type 1 or 2 diabetes $(n=3)$ were excluded. This resulted in a total of 1049 records for analysis. The flow chart of the study population is presented in Figure 4.1.The study was approved by the Institutional Review Board of Hospital Gelderse Vallei Ede.

\section{Diagnosis of GDM}

During the study period, a universal two-step screening method was used by the hospital to diagnose GDM. As first step of screening, women underwent a 50-grams Glucose Challenge Test (GCT) between 22 and 28 weeks of gestation. Those women testing positive (1-hour glucose value $\geq 7.8 \mathrm{mmol} / \mathrm{L}$ ) underwent a fasting 75-grams Oral Glucose Tolerance Test (OGTT). GDM was diagnosed if at least one test value from the OGTT was abnormal (fasting glucose plasma $\geq 6.0 \mathrm{mmol} / \mathrm{L}$ or 2 -hour plasma glucose $\geq 7.8 \mathrm{mmol} / \mathrm{L}$ ). BGDM was defined as an abnormal GCT result and a normal OGTT result.

Women referred to the hospital by local midwives for glucose screening underwent a selective onestep screening using the fasting $75 \mathrm{~g}$ OGTT (31\% of the women included for analysis). Selection was based on the presence of one or more risk factors for GDM (history of GDM, previous macrosomic infant, $\mathrm{BMI} \geq 30 \mathrm{~kg} / \mathrm{m}^{2}$, family history of diabetes, ethnic minority, unexplained intra-uterine death, and polycystic ovary syndrome). GDM was diagnosed if at least one test value from the OGTT was abnormal (fasting glucose plasma $\geq 6.0 \mathrm{mmol} / \mathrm{L}$ or 2 -hour plasma glucose $\geq 7.8 \mathrm{mmol} / \mathrm{L}$ ). 


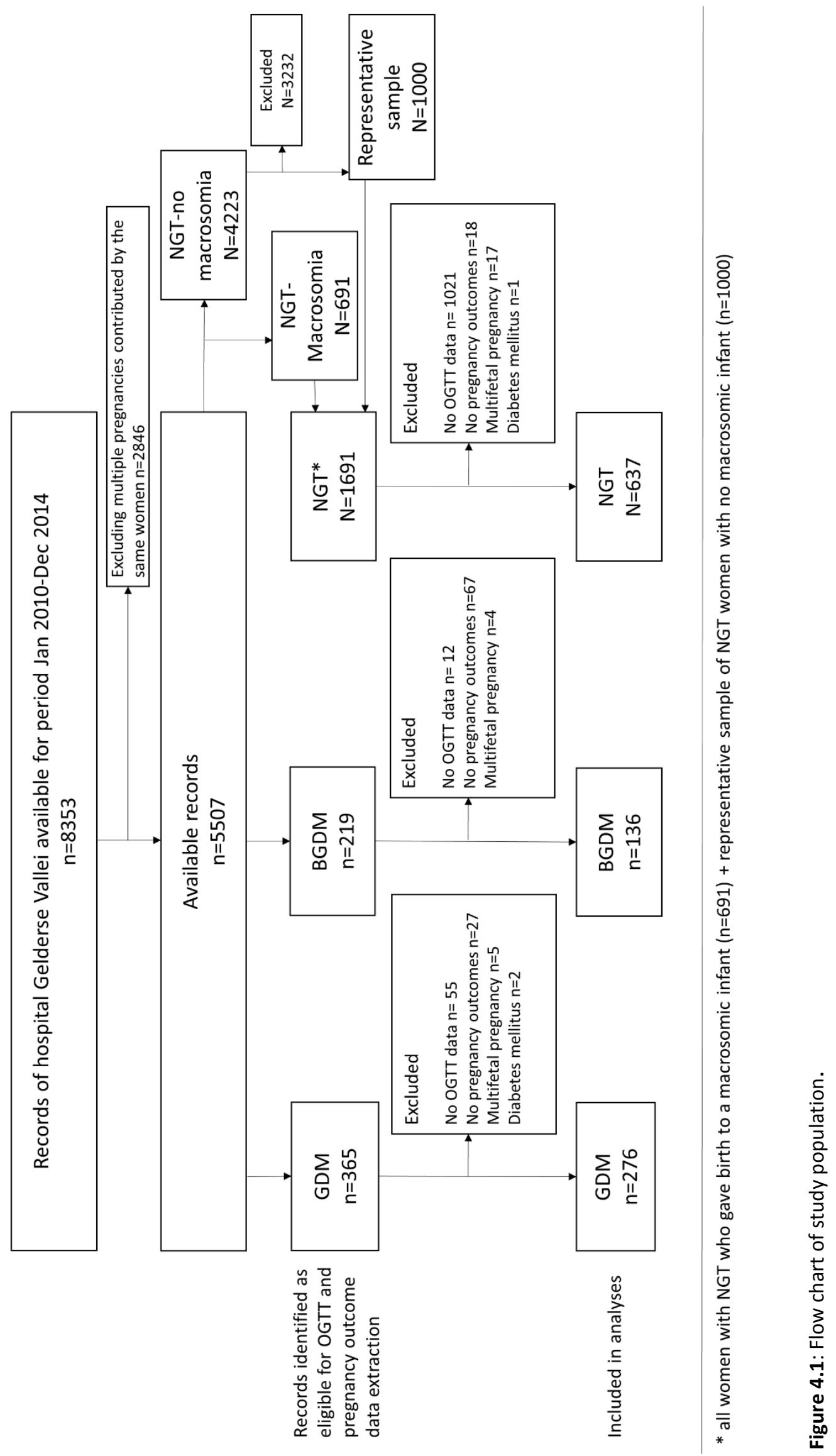


Women diagnosed with GDM received nutritional counselling to normalize glucose levels (1 hour postprandial glucose $\leq 7.0 \mathrm{mmol} / \mathrm{L}$ ). Glucose levels were measured twice weekly with a provided selfmonitoring device provided by the hospital and discussed with medical staff during a weekly consult by phone. Insulin therapy was started if dietary measures were judged as insufficient by the medical staff, i.e. 1-hour postprandial glucose $>7.0 \mathrm{mmol} / \mathrm{L}$ on multiple occasions. Women with BGDM did not receive counselling or treatment.

\section{Outcomes}

Information for the study was obtained from reviewing electronic medical files. Macrosomia was defined as a birthweight of $4000 \mathrm{~g}$ or higher. Maternal characteristics recorded were age, parity and smoking habits. Maternal smoking was defined as women who reported smoking at any time during pregnancy. Included pregnancy characteristics were gestational age at delivery, sex of infant, birthweight, birthweight percentile, Apgar score 5 minutes after birth, mode of delivery and pregnancy complications as recorded in the medical file (pregnancy hypertension or preeclampsia). Adverse pregnancy outcomes were defined as unscheduled Caesarean section (grade 1, 2 or 3 according to the classification of Lucas et al. (2000) [12]), total Caesarean section, assisted vaginal delivery (vacuum or forceps), shoulder dystocia (any prolonged head-to-body delivery time and/or the use of obstetric manoeuvres), laceration (second degree perineal tear, vaginal laceration or cervical tear, episiotomy, anal sphincter tear), and postpartum haemorrhage ( $\geq 1000 \mathrm{ml}$ blood loss).

\section{Statistical analysis}

Descriptive statistics included means and SDs for continuous variables and percentages for categorical variables. Differences between groups were tested with Chi-square test for categorical values and ANOVA for continuous values, including post-hoc comparisons with a Bonferroni correction. Cox proportional hazard models with robust variance estimates were fitted to estimate prevalence ratios as described by Barros and Hirakata [13] to assess associations of glucose tolerance status with adverse pregnancy outcomes. The first model resulted in crude prevalence ratios, weighted to account for oversampling of macrosomia in the NGT group (model 1). The adjusted model included the covariates maternal age, gestational age and parity (model 2). To assess if macrosomia mediated the association between adverse pregnancy outcomes and glucose tolerance status, macrosomia (yes/no) was additionally added to the model (model 3 ). The outcomes assisted vaginal delivery, shoulder dystocia and laceration only occur in vaginal births, therefore women who underwent a Caesarean section ( $n=307)$ were excluded from the analysis of these outcomes. Additionally, to describe the association between macrosomia and adverse pregnancy outcomes crude prevalence ratios were estimated (model 1). The adjusted model included the covariates maternal age, gestational age, parity (model 2) and glucose tolerance status (model 3). BMI is an important risk factor for GDM and macrosomia. 
Unfortunately, information on BMI was limited and pre-pregnancy body weight was not routinely recorded and only available for 358 women. In a sensitivity analysis we additionally added body weight to model 2 ( $n=358)$. Furthermore, a sensitivity analysis excluding women who underwent a one-step GDM screening ( $n=325$ ), was performed to assess the effect of possible undiagnosed BGDM in the NGT group. All statistical analyses were carried out using SAS software version 9.3 (SAS Institute Inc., Cary, NC, USA). A two-sided $p$-value of $<0.05$ was considered significant.

\section{Results}

Of the 1049 women included in the study, 136 had BGDM (12.7\%) and 276 women were diagnosed with GDM (26.3\%). Forty-seven mothers with GDM gave birth to a macrosomic infant (17.0\%), whereas 34 mothers in the BGDM group gave birth to a macrosomic infant (25.0\%). Women in the NGT group were the youngest $(p<0.05)$, whereas women with GDM had the lowest gestational age at birth $(p<0.05)$ (Table 4.1). Mode of delivery differed significantly between the groups, with highest rates of spontaneous delivery in the NGT group, highest rates of induced labour in the GDM group and highest rates of unscheduled Caesarean section in the BGDM group.

Compared to women with NGT, women with untreated BGDM had a higher risk of unscheduled Caesarean section ( $P R=1.90,95 \% \mathrm{Cl} 1.20-2.99)$, total Caesarean section ( $P R=1.51,95 \% \mathrm{Cl}$ 1.14-2.00) and laceration ( $P R=1.24,95 \% \mathrm{Cl}$ 1.06-1.46) (Table 4.2). PRs for women with GDM were lower and nonsignificant, except for a higher risk of laceration compared to women with NGT (PR=1.28, 95\% Cl 1.141.45). A higher risk of Caesarean section for women with GDM ( $P R=1.33,95 \% \mathrm{Cl} 1.04-1.70)$ was no longer significant after adjustment for maternal age, gestational age at birth and parity ( $P R=1.15$, $95 \% \mathrm{Cl}$ 0.89-1.49).

Giving birth to a macrosomic infant was associated with a higher risk of shoulder dystocia ( $P R=6.67$, 95\% $\mathrm{Cl}$ 3.46-12.83), unscheduled Caesarean section ( $\mathrm{PR}=1.55,95 \% \mathrm{Cl} 1.13-2.12$ ) and total Caesarean section ( $\mathrm{PR}=1.40,95 \% \mathrm{Cl}$ 1.13-1.73), regardless of glucose tolerance during pregnancy (Table 4.3). Macrosomia was not associated with assisted vaginal delivery, laceration or post-partum haemorrhage. Macrosomia did not mediate associations observed for glucose tolerance status and adverse pregnancy outcomes, as adding macrosomia to the model did not change prevalence ratios (model 3, Table 4.2). The only exception was attenuation of the non-significant association of BGDM and GDM with shoulder dystocia after adding macrosomia to the model. 
Table 4.1: Maternal and pregnancy characteristics of the total study population and according to glucose tolerance status during pregnancy.

\begin{tabular}{|c|c|c|c|c|c|}
\hline & Total $(n=1049)$ & $\begin{array}{l}\text { NGT\# } \\
(n=637)\end{array}$ & $\begin{array}{l}\text { BGDM } \\
(n=136)\end{array}$ & $\begin{array}{l}\text { GDM } \\
(n=276)\end{array}$ & p-value ${ }^{\wedge}$ \\
\hline Age (yrs.) & $30.9(4.7)$ & $29.6(4.0)^{a}$ & $32.3(5.1)^{b}$ & $31.9(5.3)^{b}$ & $<0.001$ \\
\hline Maternal smoking (\%) & 20.4 & 22.8 & 20.7 & 17.4 & 0.33 \\
\hline \multicolumn{6}{|l|}{ Missing=116 } \\
\hline Nulliparous (\%) & 45.9 & 49.3 & 39.6 & 44.5 & 0.18 \\
\hline Gestational age (weeks) & $39.0(1.4)$ & $39.3(1.1)^{a}$ & $38.9(1.6)^{b}$ & $38.5(1.7)^{c}$ & $<0.001$ \\
\hline Sex (\% boy) & 53.2 & 53.1 & 58.8 & 50.6 & 0.29 \\
\hline Birthweight (grams) & $3519(463)$ & $3533(370)$ & $3541(633)$ & $3489(550)$ & 0.41 \\
\hline Birthweight >90th percentile (\%) & 10.5 & 9.2 & 14.7 & 10.1 & 0.20 \\
\hline Mode of delivery (\%) & & a & $\mathrm{b}$ & $\mathrm{b}$ & $<0.001$ \\
\hline - Spontaneous & 43.7 & 52.9 & 33.1 & 37.0 & \\
\hline - Induced & 27.1 & 23.4 & 27.9 & 31.5 & \\
\hline - Elective Caesarean section & 15.5 & 12.6 & 19.9 & 17.0 & \\
\hline - Unscheduled Caesarean section & 13.7 & 11.1 & 19.1 & 14.5 & \\
\hline Pregnancy hypertension (\%) & 11.6 & 9.1 & 14.7 & 13.4 & 0.11 \\
\hline Preeclampsia (\%) & 4.8 & 5.6 & 5.9 & 3.3 & 0.32 \\
\hline Apgar score < $<*(\%)$ & 2.7 & $2.1^{\mathrm{a}}$ & $5.9^{b}$ & $1.8^{\mathrm{a}}$ & 0.04 \\
\hline
\end{tabular}

Data are presented as means (sd) for continuous variables and as percentages for categorical variables.

GDM, gestational diabetes mellitus; BGDM, borderline gestational diabetes; NGT, normal glucose tolerance.

\# Weighted to account for oversampling of macrosomia

$\wedge p$-value for differences between groups; Chi-square test for categorical variables and ANOVA for continuous variables.

$a, b, c$ Mean values with unlike superscript letters were significantly different $(p<0.05)$ in post hoc testing (Bonferroni corrected).

* Apgar score 5 minutes after birth.

Sensitivity analyses, in 358 women for which body weight was available, showed similar associations between glucose tolerance status and adverse pregnancy outcomes. The association between unscheduled Caesarean section and BGDM attenuated slightly, but remained significant (data not shown). Furthermore, excluding women screened with a one-step screening $(n=325)$, did not change results (data not shown). 
Table 4.2: Crude and adjusted prevalence ratios of adverse pregnancy outcomes according to glucose tolerance status during pregnancy.

\begin{tabular}{|c|c|c|c|}
\hline & $\begin{array}{c}\text { NGT } \\
\mathrm{n}=637\end{array}$ & $\begin{array}{l}\text { BGDM } \\
\mathrm{n}=136\end{array}$ & $\begin{array}{c}\text { GDM } \\
\mathrm{n}=276\end{array}$ \\
\hline \multicolumn{4}{|c|}{ Unscheduled Caesarean section } \\
\hline $\mathrm{n}$ & 80 & 26 & 40 \\
\hline Model 1 & 1.00 (ref) & $1.72(1.11-2.67)$ & $1.31(0.88-1.93)$ \\
\hline Model 2 & 1.00 (ref) & $1.90(1.20-2.99)$ & $1.43(0.96-2.13)$ \\
\hline Model 3 & 1.00 (ref) & $1.83(1.17-2.85)$ & $1.38(0.93-2.05)$ \\
\hline \multicolumn{4}{|c|}{ Caesarean section } \\
\hline $\mathrm{n}$ & 167 & 53 & 87 \\
\hline Model 1 & 1.00 (ref) & $1.65(1.26-2.16)$ & $1.33(1.04-1.70)$ \\
\hline Model 2 & 1.00 (ref) & $1.51(1.14-2.00)$ & $1.15(0.89-1.49)$ \\
\hline Model 3 & 1.00 (ref) & $1.48(1.12-1.95)$ & $1.14(0.88-1.47)$ \\
\hline \multicolumn{4}{|c|}{ Assisted vaginal delivery $\#$} \\
\hline $\mathrm{n}$ & 146 & 33 & 58 \\
\hline Model 1 & 1.00 (ref) & $0.58(0.30-1.12)$ & $0.76(0.50-1.16)$ \\
\hline Model 2 & 1.00 (ref) & $0.65(0.34-1.27)$ & $0.88(0.58-1.33)$ \\
\hline Model 3 & 1.00 (ref) & $0.66(0.34-1.28)$ & $0.91(0.60-1.38)$ \\
\hline \multicolumn{4}{|c|}{ Shoulder dystocia\# } \\
\hline $\mathrm{n}$ & 49 & 7 & 13 \\
\hline Model 1 & 1.00 (ref) & $1.73(0.77-3.89)$ & $1.41(0.73-2.71)$ \\
\hline Model 2 & 1.00 (ref) & $1.94(0.85-4.23)$ & $1.64(0.82-3.27)$ \\
\hline Model 3 & 1.00 (ref) & $1.48(0.66-3.33)$ & $1.40(0.73-2.71)$ \\
\hline \multicolumn{4}{|l|}{ Laceration\# } \\
\hline $\mathrm{n}$ & 299 & 61 & 141 \\
\hline Model 1 & 1.00 (ref) & $1.18(1.01-1.38)$ & $1.20(1.06-1.34)$ \\
\hline Model 2 & 1.00 (ref) & $1.24(1.06-1.46)$ & $1.28(1.14-1.45)$ \\
\hline Model 3 & 1.00 (ref) & $1.24(1.06-1.46)$ & $1.29(1.14-1.45)$ \\
\hline \multicolumn{4}{|c|}{ Post-partum haemorrhage } \\
\hline $\mathrm{n}$ & 49 & 7 & 16 \\
\hline Model 1 & 1.00 (ref) & $0.73(0.33-1.63)$ & $0.83(0.45-1.48)$ \\
\hline Model 2 & 1.00 (ref) & $0.66(0.28-1.53)$ & $0.73(0.39-1.37)$ \\
\hline Model 3 & 1.00 (ref) & $0.64(0.28-1.46)$ & $0.73(0.39-1.35)$ \\
\hline
\end{tabular}

GDM, gestational diabetes mellitus; BGDM, borderline gestational diabetes; NGT, normal glucose tolerance Model 1: Crude model, weighted for oversampling

Model 2: Model 1 adjusted for gestational age, maternal age and parity

Model 3: Model 2 adjusted for macrosomia

\# women undergoing a Caesarean section were excluded for this analysis $(n=307)$ 
Table 4.3: Crude and adjusted prevalence ratios of adverse pregnancy outcomes according to birthweight.

\begin{tabular}{|c|c|c|}
\hline & $\begin{array}{c}\text { Normal birth weight } \\
n=629\end{array}$ & $\begin{array}{c}\text { Macrosomia } \\
n=420\end{array}$ \\
\hline \multicolumn{3}{|c|}{ Unscheduled Caesarean section } \\
\hline $\mathrm{n}$ & 81 & 65 \\
\hline Model 1 & 1.00 (ref) & $1.20(0.89-1.63)$ \\
\hline Model 2 & 1.00 (ref) & $1.40(1.02-1.92)$ \\
\hline Model 3 & 1.00 (ref) & $1.55(1.13-2.12)$ \\
\hline \multicolumn{3}{|c|}{ Caesarean section } \\
\hline $\mathrm{n}$ & 179 & 128 \\
\hline Model 1 & 1.00 (ref) & $1.07(0.89-1.30)$ \\
\hline Model 2 & 1.00 (ref) & $1.33(1.08-1.64)$ \\
\hline Model 3 & 1.00 (ref) & $1.40(1.13-1.73)$ \\
\hline \multicolumn{3}{|c|}{ Assisted vaginal delivery ${ }^{\#}$} \\
\hline $\mathrm{n}$ & 146 & 33 \\
\hline Model 1 & 1.00 (ref) & $0.79(0.56-1.13)$ \\
\hline Model 2 & 1.00 (ref) & $0.84(0.58-1.21)$ \\
\hline Model 3 & 1.00 (ref) & $0.80(0.55-1.15)$ \\
\hline \multicolumn{3}{|c|}{ Shoulder dystocia\# } \\
\hline $\mathrm{n}$ & 14 & 55 \\
\hline Model 1 & 1.00 (ref) & $5.83(3.30-10.32)$ \\
\hline Model 2 & 1.00 (ref) & $6.29(3.35-11.81)$ \\
\hline Model 3 & 1.00 (ref) & $6.67(3.46-12.83)$ \\
\hline \multicolumn{3}{|l|}{ Laceration $\#$} \\
\hline $\mathrm{n}$ & 308 & 193 \\
\hline Model 1 & 1.00 (ref) & $0.96(0.87-1.07)$ \\
\hline Model 2 & 1.00 (ref) & $0.95(0.85-1.07)$ \\
\hline Model 3 & 1.00 (ref) & $1.00(0.89-1.12)$ \\
\hline \multicolumn{3}{|c|}{ Post-partum haemorrhage } \\
\hline n & 37 & 35 \\
\hline Model 1 & 1.00 (ref) & $1.42(0.91-2.21)$ \\
\hline Model 2 & 1.00 (ref) & $1.42(0.86-2.34)$ \\
\hline Model 3 & 1.00 (ref) & $1.32(0.78-2.21)$ \\
\hline
\end{tabular}

Model 1: Crude model

Model 2: Model 1 adjusted for gestational age, maternal age and parity Model 3: Model 2 adjusted for glucose tolerance status during pregnancy ( gestational diabetes mellitus/ borderline gestational diabetes/, normal glucose tolerance)

\# women undergoing a Caesarean section were excluded for this analysis $(n=307)$ 


\section{Discussion}

In this study, we found a high prevalence of macrosomia in the GDM and the BGDM group. Untreated BGDM was significantly associated with a higher risk of unscheduled Caesarean section, total Caesarean section and laceration, whereas treated GDM was associated with a higher risk of laceration compared to NGT. Macrosomia was associated with a higher risk of shoulder dystocia, unscheduled Caesarean section and total Caesarean section. We did not observe a mediating effect of macrosomia in the association of glucose tolerance status and adverse pregnancy outcomes.

Results of the HAPO study have well established that maternal glucose levels are linearly associated with risk of adverse pregnancy outcomes [3], whereas two large RCTs showed that treatment of GDM lowered risk of adverse pregnancy outcomes $[4,5]$. However, few studies have investigated whether women with treated GDM still have a higher risk of adverse pregnancy outcomes compared to NGT. We observed that women with treated GDM had more often an induction of labour for delivery and scheduled for Caesarean section. Furthermore, women with GDM had a higher risk of laceration than women with NGT, but not of other adverse pregnancy outcomes. This was also observed by another Dutch study, investigating the impact of different GDM diagnostic criteria on pregnancy outcomes [14]. A possible explanation for the higher rate of induction of labour is that induction of labour at 38-39 weeks of gestation is recommended for women with GDM, especially for those receiving insulin therapy. Thus, in our study, GDM treatment appeared to be quite successful in reducing risk of adverse pregnancy outcomes. GDM treatment in hospital Gelderse Vallei in 2010-2014 consisted of nutritional counselling by a specialized dietician and phone-based consults with medical staff. Success of GDM treatment in other populations might depend on the degree of abnormal glucose tolerance, timely diagnosis and type and intensity of GDM treatment.

At diagnosis, women with BGDM had an abnormal result on the 50g GTT screening, but a normal result on the subsequent fasting 75g OGTT confirmation test. These women received no counselling, treatment or subsequent testing. It is likely, however, that some of these women had increased maternal glucose levels throughout their pregnancy, which possibly exacerbated into uncontrolled GDM later in their pregnancy. This is supported by the fact that prevalence rates of macrosomia were higher in the BGDM group than in the treated GDM group (25\% vs. $17 \%$ ). However, it should be noted that women with GDM receiving insulin were often scheduled for a delivery at 38-39 weeks of gestation to prevent foetal complications. When looking at birth weight taking into account their gestational age (i.e. birth weight percentile), infants of women with BGDM were more often, although not statistically significantly, in the 90th birthweight percentile than infants of women with NGT or GDM, possibly due to an increased growth rate due to higher maternal glucose levels in BGDM. 
Furthermore, women with BGDM had a higher risk of laceration, an unscheduled Caesarean section and total Caesarean section. These results imply that women in the BGDM group could benefit from a second GDM screening later in pregnancy and treatment consisting of dietary counselling. A metaanalysis of four RCTs showed that dietary counselling and metabolic monitoring could reduce risk of macrosomia in women with BGDM [15]. However, a factor to take into account is that diagnosis of (B)GDM could significantly compromise quality of life [24]. Several studies observed that women with GDM struggle to adapt to a new complex behavioural regimen of diet and exercise, worry more about health and experienced more physical health problems during pregnancy [16-19].

Our finding of the increased risk of the BGDM group is especially relevant in relation to the discussion about the diagnostic criteria [20-22]. The 1999 WHO diagnostic criteria were used during the study period to define GDM [23], with a lower 2-h glucose cut-off value than the new International Association of Diabetes and Pregnancy Study Groups (IADPSG) criteria [24] ( $\geq 7.8 \mathrm{mmol} / \mathrm{L}$ versus $\geq 8.5$ ), but a higher fasting glucose cut-off value ( $\geq 6.1 \mathrm{mmol} / \mathrm{L}$ versus $\geq 5.1 \mathrm{mmol} / \mathrm{L})$. In our study, women with treated GDM still had higher risk of macrosomia and laceration compared to women with NGT, but women with BGDM did worse. This supports the use of the WHO 2-hour glucose cut-off value, i.e. $\geq 7.8$ $\mathrm{mmol} / \mathrm{L}$, as was also concluded by Koning et al. investigating the impact of different GDM diagnostic criteria on pregnancy outcomes [14].

The most pronounced adverse pregnancy outcome associated with GDM is macrosomia [3, 25-27]. As mentioned above, the rate of macrosomia was almost doubled in the GDM group (17\%), compared to the overall $10 \%$ prevalence in the hospital during the study period. Similar findings were reported by Koning et al. who observed a high prevalence of large for gestational age (LGA) infants in Dutch women with GDM living in the Northern parts of the Netherlands [28]. We found that women who give birth to a macrosomic infant have a higher risk of shoulder dystocia, unscheduled and total Caesarean section. This is in line with other studies who showed that macrosomia is associated with an increased risk of delivery-related adverse pregnancy outcomes, including shoulder dystocia, brachial plexus [2935]. Therefore, we investigated if macrosomia was a mediator in the association between GDM and adverse pregnancy outcomes. Adding macrosomia to our adjusted model did not substantially change prevalence ratios, except for attenuation of the non-significant association of BGDM and GDM with shoulder dystocia. Therefore, we conclude that risk of macrosomia did not mediate associations between glucose tolerance status and adverse pregnancy outcomes.

The strength of our study is that we could define a BGDM group. Studies relying on selective one-step screening cannot identify this group. Furthermore, we are, to our knowledge, the first study to include macrosomia as a mediator in the association between GDM and adverse pregnancy outcomes. 
However, some limitations should also be considered. In this study, we used medical records from one general hospital. Some information on maternal characteristics (e.g. smoking) was self-reported by women and information of pre-pregnancy weight was only available for 358 women, which could have led to misclassification. In addition, we could only include women who delivered in the hospital and not women who delivered at home. In the Netherlands, home delivery is common among women with an uncomplicated pregnancy and the NGT group in our study might thus comprise relatively more complicated pregnancies and thus higher rates of adverse pregnancy outcomes. Approximately 18 percent of the pregnant women in the Netherlands delivered at home in the period 2011-2013 [36]. Furthermore, we only included women who underwent glucose tolerance screening, which resulted in exclusion of 1088 records. It is therefore likely that uncomplicated pregnancies are underrepresented in our study and that our results are possibly not representative for the general population. This could have affected the prevalence of adverse pregnancy outcomes, but most likely did not affect the prevalence ratios observed. In addition, this strict inclusion was necessary to prevent undiagnosed GDM and BGDM in the normal glucose tolerance group and avoid misclassification. Thirty-one percent of the included women were diagnosed with a one-step screening method, leaving women with BGDM undiagnosed and thus misclassified as normal glucose tolerance. However, in a sensitivity analysis excluding women screened with a one-step screening, results did not change substantially (data not shown).

\section{Conclusions}

In conclusion, high rates of macrosomia were observed in the GDM and the BGDM group. Macrosomia was associated with a higher risk of shoulder dystocia, unscheduled Caesarean section and total Caesarean section, but was not a mediator in the association of glucose tolerance status and adverse pregnancy outcomes. Women with treated GDM had a higher risk of laceration compared to women with NGT. Women with untreated BGDM had a higher risk of unscheduled Caesarean section, total Caesarean section and laceration. Screening and treating BGDM to reduce risk of these adverse pregnancy outcomes warrants further research.

Conflicts of interest: The authors report no conflicts of interest.

Funding: This research did not receive any specific grant from funding agencies in the public, commercial, or not-for-profit sectors. 


\section{References}

1. Feig, D.S., J. Hwee, B.R. Shah, et al., Trends in incidence of diabetes in pregnancy and serious perinatal outcomes: a large, population-based study in Ontario, Canada, 1996-2010. Diabetes Care, 2014. 37(6): p. 1590-6.

2. International Diabetes Federation, IDF diabetes atlas. 2015: Seventh Edition.

3. Hapo Study Cooperative Research Group, B.E. Metzger, L.P. Lowe, et al., Hyperglycemia and adverse pregnancy outcomes. N. Engl. J. Med., 2008. 358(19): p. 1991-2002.

4. Crowther, C.A., J.E. Hiller, J.R. Moss, et al., Effect of treatment of gestational diabetes mellitus on pregnancy outcomes. N. Engl. J. Med., 2005. 352(24): p. 2477-86.

5. Landon, M.B., C.Y. Spong, E. Thom, et al., A multicenter, randomized trial of treatment for mild gestational diabetes. N. Engl. J. Med., 2009. 361(14): p. 1339-48.

6. Poolsup, N., N. Suksomboon, and M. Amin, Effect of treatment of gestational diabetes mellitus: a systematic review and meta-analysis. PLoS One, 2014. 9(3): p. e92485.

7. Dodd, J.M., C.A. Crowther, G. Antoniou, et al., Screening for gestational diabetes: the effect of varying blood glucose definitions in the prediction of adverse maternal and infant health outcomes. Aust. N. Z. J. Obstet. Gynaecol., 2007. 47(4): p. 307-12.

8. Yesildager, E., G. Koken, A.N. Gungor, et al., Perinatal outcomes of borderline diabetic pregnant women. J. Obstet. Gynaecol., 2014. 34(8): p. 666-8.

9. Crowther, C.A., W.M. Hague, P.F. Middleton, et al., The IDEAL study: investigation of dietary advice and lifestyle for women with borderline gestational diabetes: a randomised controlled trial - study protocol. BMC Pregnancy Childbirth, 2012. 12: p. 106.

10. Ju, H., A.R. Rumbold, K.J. Willson, et al., Borderline gestational diabetes mellitus and pregnancy outcomes. BMC Pregnancy Childbirth, 2008. 8: p. 31.

11. Kc, K., S. Shakya, and H. Zhang, Gestational diabetes mellitus and macrosomia: a literature review. Ann. Nutr. Metab., 2015. 66 Suppl 2: p. 14-20.

12. Lucas, D.N., S.M. Yentis, S.M. Kinsella, et al., Urgency of caesarean section: a new classification. J. R. Soc. Med., 2000. 93(7): p. 346-50.

13. Barros, A.J. and V.N. Hirakata, Alternatives for logistic regression in cross-sectional studies: an empirical comparison of models that directly estimate the prevalence ratio. BMC Med. Res. Methodol., 2003. 3: p. 21.

14. Koning, S.H., J.J. van Zanden, K. Hoogenberg, et al., New diagnostic criteria for gestational diabetes mellitus and their impact on the number of diagnoses and pregnancy outcomes. Diabetologia, 2017.

15. Han, S., C.A. Crowther, and P. Middleton, Interventions for pregnant women with hyperglycaemia not meeting gestational diabetes and type 2 diabetes diagnostic criteria. The Cochrane database of systematic reviews, 2012. 1: p. CD009037.

16. Carolan, M., Women's experiences of gestational diabetes self-management: a qualitative study. Midwifery, 2013. 29(6): p. 637-45.

17. Evans, M.K. and B. O'Brien, Gestational diabetes: the meaning of an at-risk pregnancy. Qual. Health Res., 2005. 15(1): p. 66-81.

18. Persson, M., A. Winkvist, and I. Mogren, 'From stun to gradual balance'--women's experiences of living with gestational diabetes mellitus. Scand. J. Caring Sci., 2010. 24(3): p. 454-62.

19. Sjogren, B., N. Robeus, and U. Hansson, Gestational diabetes: a case-control study of women's experience of pregnancy, health and the child. J. Psychosom. Res., 1994. 38(8): p. 815-22.

20. Kalter-Leibovici, O., L.S. Freedman, L. Olmer, et al., Screening and diagnosis of gestational diabetes mellitus: critical appraisal of the new International Association of Diabetes in Pregnancy Study Group recommendations on a national level. Diabetes Care, 2012. 35(9): p. 1894-6.

21. Reece, E.A. and T. Moore, The diagnostic criteria for gestational diabetes: to change or not to change? Am. J. Obstet. Gynecol., 2013. 208(4): p. 255-9.

22. Visser, G.H. and H.W. de Valk, Is the evidence strong enough to change the diagnostic criteria for gestational diabetes now? Am. J. Obstet. Gynecol., 2013. 208(4): p. 260-4.

23. World Health Organization, Definition, diagnosis and classification of diabetes mellitus and its complications. 1999, World Health Organization: Geneva, Switzerland.

24. International Association of Diabetes Pregnancy Study Groups Consensus Panel, International association of diabetes and pregnancy study groups recommendations on the diagnosis and classification of hyperglycemia in pregnancy. Diabetes Care, 2010. 33(3): p. 676-82.

25. Schmidt, M.I., B.B. Duncan, A.J. Reichelt, et al., Gestational diabetes mellitus diagnosed with a 2-h 75-g oral glucose tolerance test and adverse pregnancy outcomes. Diabetes Care, 2001. 24(7): p. 1151-5.

26. Sermer, M., C.D. Naylor, D.J. Gare, et al., Impact of increasing carbohydrate intolerance on maternal-fetal outcomes in 3637 women without gestational diabetes. The Toronto Tri-Hospital Gestational Diabetes Project. Am. J. Obstet. Gynecol., 1995. 173(1): p. 146-56.

27. Xiong, X., L.D. Saunders, F.L. Wang, et al., Gestational diabetes mellitus: prevalence, risk factors, maternal and infant outcomes. Int. J. Gynaecol. Obstet., 2001. 75(3): p. 221-8.

28. Koning, S.H., K. Hoogenberg, K.A. Scheuneman, et al., Neonatal and obstetric outcomes in diet-and insulin-treated women with gestational diabetes mellitus: a retrospective study. BMC Endocr. Disord., 2016. 16(1): p. 52. 
29. Aranha, A., U.H. Malabu, V. Vangaveti, et al., Macrosomia in non-gestational diabetes pregnancy: glucose tolerance test characteristics and feto-maternal complications in tropical Asia Pacific Australia. Asian Pac J Trop Biomed, 2014. 4(6): p. 436-40.

30. Boulet, S.L., G.R. Alexander, H.M. Salihu, et al., Macrosomic births in the united states: determinants, outcomes, and proposed grades of risk. Am. J. Obstet. Gynecol., 2003. 188(5): p. 1372-8.

31. Esakoff, T.F., Y.W. Cheng, T.N. Sparks, et al., The association between birthweight $4000 \mathrm{~g}$ or greater and perinatal outcomes in patients with and without gestational diabetes mellitus. Am. J. Obstet. Gynecol., 2009. 200(6): p. 672 e1-4.

32. Stotland, N.E., A.B. Caughey, E.M. Breed, et al., Risk factors and obstetric complications associated with macrosomia. Int. J. Gynaecol. Obstet., 2004. 87(3): p. 220-6.

33. Benedetti, T.J. and S.G. Gabbe, Shoulder dystocia. A complication of fetal macrosomia and prolonged second stage of labor with midpelvic delivery. Obstet. Gynecol., 1978. 52(5): p. 526-9.

34. Nesbitt, T.S., W.M. Gilbert, and B. Herrchen, Shoulder dystocia and associated risk factors with macrosomic infants born in California. Am. J. Obstet. Gynecol., 1998. 179(2): p. 476-80.

35. Nocon, J.J., D.K. McKenzie, L.J. Thomas, et al., Shoulder dystocia: an analysis of risks and obstetric maneuvers. Am. J. Obstet. Gynecol., 1993. 168(6 Pt 1): p. 1732-7; discussion 1737-9.

36. Statistics Netherlands, Delivery and Births: 1989-2013. 2015, Statistics Netherlands: Den Haag/Heerlen. 



\section{Chapter 5}

Pre-pregnancy dietary carbohydrate quantity and quality, and risk of developing gestational diabetes: the Australian Longitudinal

Study on Women's Health

Moniek Looman, Daniëlle A.J.M. Schoenaker, Sabita S. Soedamah-Muthu, Anouk Geelen, Edith J.M. Feskens, Gita D. Mishra 


\section{Abstract}

Background: Carbohydrate quantity and quality affect postprandial glucose response, glucose metabolism and risk of type 2 diabetes. The aim of the present study was to examine the association of pre-pregnancy dietary carbohydrate quantity and quality with risk of developing gestational diabetes (GDM).

Methods: We used data from the Australian Longitudinal Study on Women's Health that included 3607 women aged 25-30 years without diabetes who were followed-up between 2003 and 2015. We examined carbohydrate quantity (total carbohydrate intake and a low-carbohydrate diet (LCD) score), and carbohydrate quality (fibre, total sugar intake, glycaemic index, glycaemic load and intake of carbohydrate-rich food groups). Relative risks (RR) for development of GDM were estimated using multivariable regression models with generalized estimating equations.

Results: During 12 years follow-up, 285 cases of GDM were documented in 6263 pregnancies (4.6\%). The LCD score, reflecting relatively high fat and protein intake and low carbohydrate intake, was positively associated with GDM risk (RR 1.54 [1.10-2.15], highest quartile vs. lowest quartile). Women in the quartile with highest fibre intake had a 33\% lower risk of GDM (RR 0.67 [0.45-0.96]). Higher intakes of fruit (0.95 per 50g/day [0.90-0.99]) and fruit juice (0.89 per 100g/day [0.80-1.00]) were inversely associated with GDM, whereas cereal intake was associated with a higher risk of GDM (RR 1.05 per 20g/day [1.01-1.07]).

Conclusions: A relatively low carbohydrate and high fat and protein intake may increase risk of GDM, whereas higher fibre intake could decrease risk of GDM. It is especially important to take the source of carbohydrates into account. 


\section{Introduction}

Gestational diabetes mellitus (GDM) is one of the most common metabolic complications during pregnancy and prevalence has continued to increase worldwide [1, 2]. GDM is associated with shortterm adverse perinatal and pregnancy outcomes such as increased risk of macrosomia, Caesarean section and neonatal hyperglycaemia [3]. Furthermore, mothers with GDM and their offspring are at increased risk of developing type 2 diabetes [4-6]. Few modifiable risk factors for GDM have been identified, with diet as an important one as it is relatively easy to modify $[7,8]$.

GDM is characterized by an impaired ability of the body to respond to increases in postprandial blood glucose [9]. Fat and protein intake affect postprandial glucose homeostasis indirectly via affecting insulin secretion, sensitivity or resistance [10]. However, carbohydrate is the only macronutrient that directly affects postprandial blood glucose and long-term postprandial response. Therefore, prepregnancy carbohydrate intake might be a significant dietary factor in the prevention of GDM. Epidemiological studies have shown that dietary fibre, glycaemic index (GI) and glycaemic load (GL) are consistently associated with risk of type 2 diabetes $[11,12]$. However, studies on the role of prepregnancy carbohydrate intake in relation to GDM incidence are limited and, up to date, only performed using the Nurses' Health Study data $[13,14]$.

More studies in other populations are needed to confirm the possible relation between pre-pregnancy carbohydrate intake and GDM prevention. The association of carbohydrate intake and GDM risk can be investigated by examining the relationship between total carbohydrate intake (quantity) and GDM. However, carbohydrate quality (type of carbohydrate) might be more important as different types of carbohydrates have different rates of digestion and absorption, and thus might have different effects on blood glucose levels [15]. Therefore, we aimed to examine the associations between pre-pregnancy dietary carbohydrate quantity and quality, and GDM incidence. Carbohydrate quantity was examined by investigating total carbohydrate intake and a low-carbohydrate diet score [13, 16]. Carbohydrate quality was investigated by examining fibre, and total sugar intake, GI, GL and intake of carbohydraterich food groups. 


\section{Subjects and Methods}

\section{Study design and population}

The current study used data from the Australian Longitudinal Study on Women's Health (ALSWH). ALSWH is an ongoing population-based prospective cohort study investigating the role of demographic, social, physical, psychological, and behavioural factors in women's health. Full details on study design, recruitment, methods and responses have been described elsewhere $[17,18]$. Briefly, in 1996 approximately 40,000 women across three cohorts were recruited: those born in 1973-78 (1823 years), 1946-51 (45-50 years) and 1921-26 (70-75 years). Women were randomly selected from Australia's nationalized health-care system, Medicare, with intentional oversampling in rural and remote areas. Participants gave informed consent at each survey. The study was conducted according to the declaration of Helsinki and approved by the Human Research Ethics Committees of the Universities of Newcastle and Queensland.

For this study, data from the young cohort of women born in 1973-78 were used. This sample was broadly representative of Australian women of the same age at baseline [17]. Self-administered questionnaires were sent to participants every 3-4 years. Dietary intake was first collected at Survey 3 $(2003, n=9081)$ when women were 25-30 years and again at Survey $5(2009, n=8200)$. Survey 3 was used as baseline for the current analyses. Women were excluded from the current analyses if they did not report a live birth at consecutive surveys in 2006, 2009, 2012 or 2015, had missing data on diet at Survey 3 and 5, had missing data on GDM, reported implausible energy intake (ratio of reported energy intake and predicted energy requirement $<0.56$ or $>1.44$ [19]), had a history of type 1 or type 2 diabetes mellitus prior to GDM diagnosis, had a history of GDM prior to baseline (Survey 3), or had missing covariate data (Figure 5.1). A total of 3607 women were included for the analyses.

\section{Dietary assessment}

Diet was assessed using the Dietary Questionnaire for Epidemiological Studies (DQES) version 2. This 101-item food frequency questionnaire (FFQ) assesses usual food and beverage intake of the previous 12 months. The development and evaluation of this FFQ has been described elsewhere [20, 21]. Briefly, participants were asked to report their usual frequency of consumption of 74 food items and six alcoholic beverage items using a 10-point scale ranging from 'never' to 'three or more times per day' and for 21 items the number of servings of milk, bread, sugar and eggs, and the type of milk, bread, fat spread and cheese consumed. Portion size photographs were used to assess the serving sizes. Added sugar intake was assessed with the question 'On average, how many teaspoons of sugar do you usually use per day? (Include sugar taken with tea and coffee and on breakfast cereal, etc.)'. Nutrient intakes were computed using the national government food composition database of Australian foods, the 


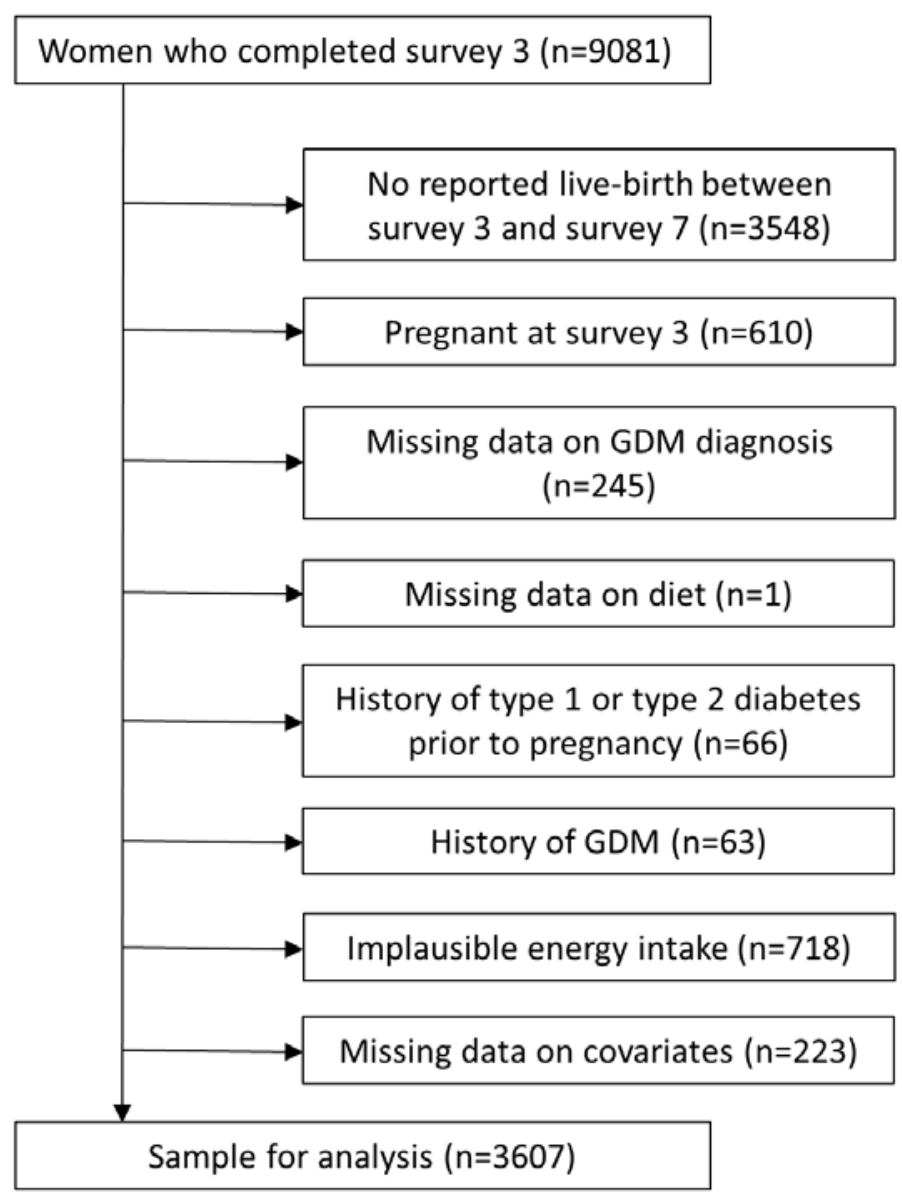

Figure 5.1: Flow chart of the study population.

NUTTAB95 [22]. Validation of the FFQ against 7 day food diaries of 63 women of reproductive age showed moderate to strong energy-adjusted correlation coefficients for a wide range of macro- and micronutrients (ranging from 0.28 for vitamin A to 0.78 for carbohydrates) [20].

\section{Carbohydrate quantity}

Carbohydrate quantity was examined by investigating total carbohydrate intake, expressed as nutrient density (percent of total energy intake) and the low-carbohydrate diet score (LCD). The LCD score is a measure of the carbohydrate content of the diet relative to fat and protein intake [16], with a low score reflecting a diet high in carbohydrate intake and a high score reflecting a low carbohydrate intake. To avoid interference of energy, energy densities were used instead of total intake in $\mathrm{g} /$ day. The LCD score was calculated by dividing the study participants into 11 equal strata each of fat, protein, and carbohydrate intake (E\%). Women in the highest strata of fat and protein intake received 10 points for that macronutrient; women in the next strata received 9 points and so on. For carbohydrate, the 
scoring was reversed, thus women in the lowest stratum received 10 points and those with the highest intake received 0 points. The points for the three macronutrients were summed to create the overall LCD score, ranging from 0 (lowest fat and protein intake and highest carbohydrate intake) to 30 (highest fat and protein intake and lowest carbohydrate intake).

\section{Carbohydrate quality}

Carbohydrate quality was examined using fibre (grams/day), and total sugar intake (grams/day), GI, GL and intake of carbohydrate-rich food groups (grams/day). Total sugar intake comprised the intake of mono- and disaccharides. The GI is a relative measure of the glycaemic impact of the carbohydrates in different foods [23]. GI values of individual food items included in the FFQ were obtained from the 2002 International table of GI and GL values [24], with glucose as reference food. If Australian figures were available, these were used. When there was more than one value available, GI values were averaged. For each person GI values of the food items were multiplied by carbohydrate intake (in grams) from that food item and summed to obtain a person's GL. The average GI for each participant was calculated by dividing GL by total carbohydrate intake [24]. Alcoholic beverages were not included in the overall Gl. Nine carbohydrate-rich food groups were comprised for additional analyses: white bread; high fibre bread (high-fibre white bread, whole meal bread, rye bread, multi-grain bread); cereal (All Bran, bran flakes, muesli, Weet-Bix, cornflakes, porridge); fruit (oranges, apples, pears, bananas, melon, pineapple, strawberries, apricots, peaches, mango, avocado, tinned fruit); fruit juice; staple products (rice, pasta); added sugar; vegetables (tomato, tomato sauce, capsicum (bell or sweet peppers), lettuce, cucumber, celery, beetroot, carrots, cabbage, cauliflower, broccoli, spinach, peas, green beans, bean sprouts, pumpkin, onion, garlic, mushrooms, zucchini, potato) and a combined vegetables and fruit group.

Dietary carbohydrate (E\%), fibre, and total sugar intake, GI, GL and LCD score were adjusted for energy using the residual method [25].

\section{Assessment of GDM}

GDM was assessed using self-reported physician diagnosis at each survey and for each live birth using the following question: 'Were you diagnosed by a doctor or treated for gestational diabetes?'. A reliability study among a subgroup of 1914 women from New South Wales demonstrated high agreement of $91 \%$ between self-reported GDM diagnosis in our study and administrative data records [26]. 


\section{Assessment of covariates}

Information on country of birth was assessed at Survey 1. Information on highest qualification completed, number of hours paid work, marital status, parity, hypertensive disorders of pregnancy, polycystic ovary syndrome, inter-pregnancy interval, smoking, physical activity and body mass index (BMI) was self-reported at Survey 3 to Survey 6 . Physical activity was assessed using validated questions on frequency and duration of walking and on moderate- and vigorous-intensity activity and was categorized as sedentary/low ( $<600$ total metabolic equivalent [MET] min/week), moderate (600 to <1200 MET min/week) or high ( $21200 \mathrm{MET} \mathrm{min} /$ week) [27]. BMI was categorized as underweight $\left(\mathrm{BMI}<18.5 \mathrm{~kg} / \mathrm{m}^{2}\right.$ ), normal weight (BMI 18.5 to $<25 \mathrm{~kg} / \mathrm{m}^{2}$ ), overweight (BMI 25 to $<30 \mathrm{~kg} / \mathrm{m}^{2}$ ) or obese $\left(\mathrm{BMI} \geq 30 \mathrm{~kg} / \mathrm{m}^{2}\right)$. Only a few women were classified as underweight $(\mathrm{n}=123,3.4 \%)$; therefore, the underweight and normal weight groups were combined as normal weight $\left(\mathrm{BMI}<25 \mathrm{~kg} / \mathrm{m}^{2}\right)$.

\section{Statistical analysis}

Baseline characteristics reported at Survey 3 are shown according to quartiles of LCD-score, our main outcome regarding carbohydrate quantity. Characteristics were compared using ANOVA or $\chi^{2}$ tests. Characteristics were weighted by area of residence to account for oversampling of women from rural and remote areas. As dietary intake can change over time the most recent reported dietary intake before the index pregnancy was used.

Generalized estimating equations (GEE) analyses were used to account for correlated observations due to multiple pregnancies by the same participant [28]. Log-Poisson models were used to estimate relative risks (RR) and $95 \%$ confidence intervals $(95 \% \mathrm{Cl})$ for associations between carbohydrate quality, quantity and GDM [29] as log-binomial models did not converge. Adjustment for time-varying covariates (education level, work status, marital status, BMI, smoking, physical activity, parity, PCOS) was performed using the value reported at the survey administered prior to the pregnancy. For pregnancy-specific covariates (hypertension during pregnancy and, if applicable, inter-pregnancy interval) the value reported for that specific pregnancy was used. Multiple gestation, alcohol intake, work status and marital status were not included in the analyses, as these were not significant confounders based on the data.

Partial correlations, adjusted for energy intake, were calculated to investigate correlations between carbohydrate-rich food groups and measures of carbohydrate quantity (carbohydrate intake (E\%), LCD score) and carbohydrate quality (fibre and total sugar intake, glycaemic index and glycaemic load). Associations between intake of carbohydrate-rich food groups and risk of GDM were investigated by comparing quartiles of intake to determine if the associations were linear (data not shown). The median intake of the quartiles was analysed as a continuous variable in multivariable models to obtain 
a p-value for linear trend. Intakes of carbohydrate-rich food groups were subsequently analysed in multivariable models with intake as a continuous variable. The association between added sugar and risk of GDM was assessed for users vs. non-users because of the large proportion of non-users and the subsequently skewed distribution.

To examine the robustness of the observed associations several sensitivity analyses were performed. First, we examined the associations combining dietary intake data from Surveys 3 and 5 to calculate long-term average dietary intake. Furthermore, to exclude the possible effect of women changing their normal diet to increase their chance of conception, all pregnancies within the first two years of followup were excluded. Additionally, we conducted a multiple imputation analysis to assess the influence of participant exclusions that resulted from missing covariate data (educational level, work, marital, smoking and alcohol status, PCOS and BMI; $\mathrm{n}=223$ ) using SAS procedures MI and MIANALYZE [30]. Finally, analyses were stratified by known risk factors for GDM including BMI $(<25,25-29.9$, or $>30$ $\mathrm{kg} / \mathrm{m}^{2}$ ), educational level (low, moderate, high) or parity (nulliparous versus parous) as these were identified as potential effect modifiers in other studies on diet and GDM [14, 31].

Statistical analyses were conducted using SAS Software Version 9.4 (SAS Institute Inc., Cary, NC, USA). A $p$ value $<0.05$ was considered statistically significant.

\section{Results}

During 12 years of follow-up (2003-2015), 285 cases of GDM (4.6\%) were reported in 6263 pregnancies among 3607 participants. Women with GDM were more often born in Asia, had a higher BMI, were more often nulliparous and more likely to have PCOS (data not shown). Women who had a prepregnancy diet with a relatively low carbohydrate intake (i.e. quartile 4 compared with quartile 1 of the LCD score) lived on average more often in rural/remote areas (Table 5.1). Furthermore, these women in the highest quartile of the LCD score were more often born in Australia, overweight or obese, current smokers, high-risk alcohol consumers, and were less physically active and less educated compared to women in the lowest quartile.

\section{Carbohydrate quantity}

Participants in the highest quartile of carbohydrate intake had a lower risk of developing GDM compared to the lowest quartile, after adjustment for socio-demographic factors (including age, country of birth, education level), reproductive factors (including parity, hypertension during pregnancy, PCOS and inter-pregnancy interval) and lifestyle factors (including smoking, energy intake 
and physical activity level) (Table 5.2). However, adjustments for protein intake, fat intake, and BMI attenuated the observations and results were no longer statistically significant. The LCD score (reflecting relatively high fat and protein intakes and a low carbohydrate intake) was significantly associated with a $54 \%$ higher risk of GDM for women in the highest quartile compared to the lowest quartile (RR 1.54, 95\% Cl 1.10-2.15) after adjustment for socio-demographic, reproductive, lifestyle and dietary factors. Additional adjustment for BMI slightly attenuated the association (RR $1.43,95 \% \mathrm{Cl}$ 1.03-2.01).

\section{Carbohydrate quality}

Total sugar intake was inversely associated with risk of developing GDM after adjustment for sociodemographic, lifestyle and reproductive factors (Table 5.2). The association was attenuated after adjustment for dietary factors and BMI and no longer statistically significant ( $\mathrm{RR} 0.83,95 \% \mathrm{Cl} 0.56$ 1.23). Women in the highest quartile of total fibre intake had a $33 \%$ lower risk of GDM compared to women in the lowest quartile (RR $0.67,95 \% \mathrm{Cl} 0.45-0.96)$ adjusted for smoking, physical activity, sociodemographic, reproductive and dietary factors. Further adjustment for $\mathrm{BMI}$ attenuated the association (RR $0.72,95 \% \mathrm{Cl} 0.50-1.05$ ). A non-significant positive trend was seen between glycaemic index and glycaemic load with development of GDM adjusted for socio-demographic, reproductive, lifestyle and dietary factors.

Table 5.1: Baseline characteristics of non-pregnant Australian women according to quartile of low carbohydrate diet (LCD) score ( $\mathrm{n}=3607)$.

\begin{tabular}{|c|c|c|c|c|c|}
\hline \multirow[b]{3}{*}{ Characteristic $^{1}$} & \multicolumn{4}{|c|}{ Low carbohydrate diet (LCD) score } & \multirow[b]{3}{*}{$p$-value ${ }^{2}$} \\
\hline & Quartile 1 & Quartile 2 & Quartile 3 & Quartile 4 & \\
\hline & $\mathrm{N}=903$ & $\mathrm{~N}=898$ & $\mathrm{~N}=904$ & $\mathrm{~N}=902$ & \\
\hline Age (yrs) & $27.5(1.5)$ & $27.6(1.4)$ & $27.5(1.5)$ & $27.5(1.5)$ & 0.53 \\
\hline Area of residence & & & & & $<0.001$ \\
\hline Urban & 77.9 & 76.4 & 70.1 & 69.7 & \\
\hline Rural/remote & 22.1 & 23.6 & 29.9 & 30.3 & \\
\hline Country of birth & & & & & $<0.001$ \\
\hline Australia & 89.2 & 90.8 & 92.8 & 92.1 & \\
\hline Asia & 3.0 & 2.1 & 1.6 & 0.3 & \\
\hline Other & 7.8 & 7.1 & 5.6 & 7.6 & \\
\hline Highest educational level & & & & & 0.001 \\
\hline Up to year 12 or equivalent & 14.4 & 17.0 & 19.5 & 24.5 & \\
\hline Trade/apprenticeship/certificate/diploma & 20.0 & 20.4 & 22.4 & 23.8 & \\
\hline University/higher degree & 65.6 & 62.6 & 58.1 & 51.6 & \\
\hline Work status & & & & & 0.34 \\
\hline No-paid job & 15.6 & 16.1 & 15.7 & 13.3 & \\
\hline Part-time & 21.8 & 19.2 & 22.5 & 22.4 & \\
\hline Full-time & 62.6 & 64.7 & 61.8 & 64.6 & \\
\hline Marital status & & & & & 0.08 \\
\hline Married/de facto & 67.5 & 69.4 & 64.1 & 64.4 & \\
\hline Separated/divorced/widowed & 2.2 & 2.0 & 3.5 & 3.0 & \\
\hline
\end{tabular}


Table 5.1 continued:

\begin{tabular}{|c|c|c|c|c|c|}
\hline Single & 30.3 & 28.6 & 32.4 & 32.6 & \\
\hline $\mathrm{BMI}\left(\mathrm{kg} / \mathrm{m}^{2}\right)$ & $23.3(4.2)$ & $23.5(4.3)$ & $23.9(4.6)$ & $24.4(5)$ & $<0.001$ \\
\hline BMI & & & & & $<0.001$ \\
\hline Normal weight $\left(<25 \mathrm{~kg} / \mathrm{m}^{2}\right)$ & 74.8 & 76.0 & 68.6 & 66.6 & \\
\hline Overweight $\left(25\right.$ to $<30 \mathrm{~kg} / \mathrm{m}^{2}$ ) & 17.6 & 15.7 & 21.1 & 21.2 & \\
\hline Obese $\left(\geq 30 \mathrm{~kg} / \mathrm{m}^{2}\right)$ & 7.6 & 8.3 & 10.3 & 12.2 & \\
\hline Physical activity & & & & & 0.04 \\
\hline Sedentary/low (<600 MET min/week) & 39.5 & 39.7 & 42.5 & 43.0 & \\
\hline Moderate (600 to <1200 MET min/week) & 22.7 & 26.4 & 26.7 & 24.6 & \\
\hline High ( $\geq 1200$ MET min/week) & 37.8 & 33.8 & 30.8 & 32.3 & \\
\hline Smoking status & & & & & $<0.001$ \\
\hline Never smoked & 68.8 & 65.6 & 59.6 & 54.6 & \\
\hline History of smoking & 17.5 & 18.1 & 17.3 & 17.5 & \\
\hline Current smoker & 13.7 & 16.3 & 23.1 & 27.9 & \\
\hline Alcohol intake status & & & & & $<0.001$ \\
\hline Non drinker & 7.5 & 4.1 & 5.4 & 3.5 & \\
\hline Low risk/rarely drinks & 90.9 & 93.8 & 91.2 & 90.7 & \\
\hline High risk/often drinks & 1.6 & 2.1 & 4.4 & 5.8 & \\
\hline Nulliparous & 79.5 & 78.6 & 78.1 & 77.9 & 0.84 \\
\hline Polycystic ovary syndrome & 9.2 & 8.9 & 8.5 & 7.9 & 0.80 \\
\hline Total energy intake (kJ/day) & $6993(1741)$ & 7044 (1761) & $7076(1654)$ & $7123(1714)$ & 0.46 \\
\hline Total fat intake (E\%) & $31.3(4.8)$ & $34.8(4.5)$ & $37.2(4.4)$ & $40.4(4.1)$ & $<0.001$ \\
\hline Total saturated fat intake (E\%) & $12.6(2.9)$ & $14.3(2.8)$ & $15.5(3)$ & $16.8(2.9)$ & $<0.001$ \\
\hline Total protein intake (E\%) & $18(2.6)$ & $19.5(2.8)$ & $20.2(2.8)$ & $21.7(3)$ & $<0.001$ \\
\hline Total carbohydrate intake (E\%) & $50.9(4.2)$ & $45.9(2.8)$ & $42.9(3.4)$ & $38.2(4.5)$ & $<0.001$ \\
\hline Total fibre intake (g/MJ) & $3.2(0.8)$ & $2.8(0.7)$ & $2.7(0.6)$ & $2.5(0.6)$ & $<0.001$ \\
\hline Total sugar intake (g) & $99.3(21.3)$ & $87.4(16.6)$ & $77(16.2)$ & $65.7(16.7)$ & $<0.001$ \\
\hline Glycaemic index & $53(3.6)$ & $52(3.8)$ & $52(3.9)$ & $51(3.8)$ & $<0.001$ \\
\hline Glycaemic load & $114(12.5)$ & $101(7.7)$ & $93(7.2)$ & $80(10.6)$ & $<0.001$ \\
\hline Low carbohydrate score (LCD) & $5.9(2.7)$ & $12.2(1.6)$ & $17.5(1.5)$ & $24.5(2.6)$ & $<0.001$ \\
\hline
\end{tabular}

\section{Carbohydrate-rich food groups}

Carbohydrate intake, LCD score, total sugar intake, fibre intake, GI and GL were associated with different carbohydrate-rich food groups as indicated by partial correlations, adjusted for energy (Table 5.3). Intake of high fibre bread, vegetables and fruit, fruit, and fruit juice was inversely linearly associated with risk of GDM, while white bread intake was positive linearly associated with GDM risk and intake of cereal, staple products and vegetables was not associated with GDM risk (Table 5.4). After additional adjustment for the other food groups (model 2), intake of vegetables and fruit, fruit and fruit juice were inversely associated with development of GDM. Intake of the combined food group fruit and vegetables was significantly associated with a 10\% lower risk of developing GDM per $100 \mathrm{~g} /$ day increment. When analysing intake of fruit and vegetables separately, only fruit intake remained inversely associated with GDM risk (RR 0.95 per 50g/day, 95\% $\mathrm{Cl} 0.90-0.99$ ). Intake of cereal was associated with a higher risk of GDM (RR 1.05 per 20g/day, 95\% Cl 1.01-1.07), but the association 
Table 5.2: Relative risk of GDM according to quartiles of dietary intakes of carbohydrate, total sugar, and fibre, dietary glycaemic index and load and low carbohydrate diet (LCD) score.

\begin{tabular}{|c|c|c|c|c|c|}
\hline & \multicolumn{4}{|c|}{ Quartiles } & \multirow[b]{2}{*}{$P$ for trend } \\
\hline & Quartile 1 & Quartile 2 & Quartile 3 & Quartile 4 & \\
\hline \multicolumn{6}{|l|}{ Carbohydrates (E\%) } \\
\hline Median & 37.5 & 42.1 & 45.5 & 50.3 & \\
\hline $\mathrm{N}$ women/pregnancies & $901 / 1541$ & $901 / 1611$ & $903 / 1601$ & $902 / 1510$ & \\
\hline GDM cases $n$ (\% pregnancies) & $90(5.8)$ & $76(4.7)$ & $65(4.1)$ & $54(3.6)$ & \\
\hline Model 1 & 1.00 & $0.81(0.60-1.08)$ & $0.71(0.52-0.97)$ & $0.63(0.45-0.88)$ & 0.004 \\
\hline Model 2 & 1.00 & $0.78(0.53-1.13)$ & $0.67(0.41-1.10)$ & $0.57(0.27-1.18)$ & 0.13 \\
\hline Model 2+BMI & 1.00 & $0.78(0.54-1.12)$ & $0.68(0.40-1.08)$ & $0.56(0.27-1.16)$ & 0.12 \\
\hline \multicolumn{6}{|l|}{ LCD score } \\
\hline Median & 6.4 & 12.2 & 17.4 & 24.0 & \\
\hline $\mathrm{N}$ women/pregnancies & $901 / 1524$ & $902 / 1602$ & $902 / 1600$ & $902 / 1537$ & \\
\hline GDM cases $n$ (\% pregnancies) & $52(3.4)$ & $70(4.4)$ & 79 (4.9) & $84(5.5)$ & \\
\hline Model 1 & 1.00 & $1.27(0.90-1.80)$ & $1.40(0.99-1.98)$ & $1.54(1.10-2.15)$ & 0.01 \\
\hline Model 2+BMla & 1.00 & $1.26(0.89-1.77)$ & $1.35(0.95-1.90)$ & $1.43(1.03-2.01)$ & 0.03 \\
\hline \multicolumn{6}{|l|}{ Total sugars (g/day) } \\
\hline Median & 59.6 & 76.1 & 89.0 & 106.2 & \\
\hline $\mathrm{N}$ women/pregnancies & $901 / 1541$ & $903 / 1606$ & $902 / 1586$ & $901 / 1530$ & \\
\hline GDM cases $\mathrm{n}$ (\% pregnancies) & $90(5.8)$ & $71(4.4)$ & $61(3.9)$ & $63(4.1)$ & \\
\hline Model 1 & 1.00 & $0.78(0.58-1.06)$ & $0.71(0.51-0.99)$ & $0.72(0.52-0.99)$ & 0.04 \\
\hline Model 2 & 1.00 & $0.83(0.61-1.13)$ & $0.78(0.54-1.13)$ & $0.83(0.56-1.24)$ & 0.33 \\
\hline Model 2+BMI & 1.00 & $0.83(0.61-1.14)$ & $0.77(0.54-1.11)$ & $0.83(0.56-1.23)$ & 0.32 \\
\hline \multicolumn{6}{|l|}{ Total dietary fibre (g/day) } \\
\hline Median & 14.5 & 17.7 & 20.6 & 24.9 & \\
\hline $\mathrm{N}$ women/pregnancies & $902 / 1554$ & $902 / 1586$ & $901 / 1552$ & $902 / 1571$ & \\
\hline GDM cases $n$ (\% pregnancies) & $88(5.7)$ & $67(4.2)$ & $72(4.6)$ & $58(3.7)$ & \\
\hline Model 1 & 1.00 & $0.77(0.56-1.04)$ & $0.83(0.61-1.12)$ & $0.62(0.45-0.87)$ & 0.01 \\
\hline Model 2 & 1.00 & $0.77(0.56-1.06)$ & $0.85(0.62-1.18)$ & $0.67(0.45-0.96)$ & 0.05 \\
\hline Model 2+BMI & 1.00 & $0.79(0.58-1.08)$ & $0.90(0.65-1.24)$ & $0.72(0.50-1.05)$ & 0.15 \\
\hline \multicolumn{6}{|l|}{ Glycaemic Index } \\
\hline Median & 47.8 & 50.8 & 53.4 & 56.7 & \\
\hline $\mathrm{N}$ women/pregnancies & $901 / 1529$ & $902 / 1618$ & $902 / 1579$ & $902 / 1537$ & \\
\hline GDM cases $n$ (\% pregnancies) & $70(4.6)$ & $70(4.3)$ & $69(4.4)$ & $76(4.9)$ & \\
\hline Model 1 & 1.00 & $0.99(0.73-1.36)$ & $1.06(0.77-1.46)$ & $1.25(0.90-1.73)$ & 0.19 \\
\hline Model 2 & 1.00 & $1.06(0.77-1.46)$ & $1.16(0.83-1.63)$ & $1.41(0.99-2.02)$ & 0.06 \\
\hline Model 2+BMI & 1.00 & $1.02(0.74-1.40)$ & $1.13(0.80-1.58)$ & $1.35(0.94-1.94)$ & 0.09 \\
\hline \multicolumn{6}{|l|}{ Glycaemic Load } \\
\hline Median & 80.5 & 92.2 & 100.8 & 114.1 & \\
\hline $\mathrm{N}$ women/pregnancies & $901 / 1547$ & $902 / 1605$ & $902 / 1555$ & $902 / 1556$ & \\
\hline GDM cases $\mathrm{n}$ (\% pregnancies) & $89(5.8)$ & $66(4.1)$ & $68(4.4)$ & $62(4.0)$ & \\
\hline Model 1 & 1.00 & $0.78(0.57-1.06)$ & $0.83(0.62-1.13)$ & $0.78(0.57-1.07)$ & 0.17 \\
\hline Model 2 & 1.00 & $0.95(0.66-1.36)$ & $1.15(0.76-1.75)$ & $1.29(0.77-2.18)$ & 0.28 \\
\hline Model 2+BMI & 1.00 & $0.94(0.65-1.35)$ & $1.10(0.72-1.68)$ & $1.26(0.73-2.14)$ & 0.35 \\
\hline
\end{tabular}

Model 1: adjusted for age at pregnancy (years), country of birth (Australia, Asia, or other), educational level (low, medium, or high), total energy intake (kJ/day), physical activity (low, medium, or high), smoking (current, former, or never), polycystic ovarian syndrome (yes or no), hypertension during pregnancy (yes or no), parity $(0,1$, or $\geq 2$ ), inter-pregnancy interval (not applicable [first pregnancy], $<18$ months, 18-60 months, $>60$ months)

Model 2: model $1+$ additional adjustments for fat and protein intake (E \%)

Model 2+BMI: model 2 + additional adjustments for BMI (normal weight, overweight, or obese)

a Not adjusted for fat and protein intake, as these are part of the score 
between cereal and GDM was slightly U-shaped ( $p$ for linear trend 0.11). Furthermore, women who consumed added sugar ( $n=2154$, median intake $15.5 \mathrm{~g} /$ day) had a $29 \%$ higher risk of GDM than women who did not consume added sugar, which slightly attenuated after adjustment for the other food groups and $\mathrm{BMI}$, and was not statistically significant (RR 1.25, 95\% Cl 0.98-1.59).

\section{Sensitivity analyses}

Associations observed between fibre, LCD score and development of GDM persisted in sensitivity analyses, as well as associations between carbohydrate-rich food groups and GDM risk (data not shown). Interaction terms for $\mathrm{BMI}$, educational level and parity were not significant. Additional stratification did not change the results materially.

Table 5.3: Partial correlations between carbohydrate intake, LCD score, total sugar intake, fibre intake, glycaemic index, glycaemic load and carbohydrate-rich food groups, adjusted for energy intake.

\begin{tabular}{lcccccc}
\hline $\begin{array}{l}\text { Carbohydrate-rich } \\
\text { food groups }\end{array}$ & $\begin{array}{c}\text { Carbohydrate } \\
\text { intake }\end{array}$ & LCD score & $\begin{array}{c}\text { Total sugar } \\
\text { intake }\end{array}$ & $\begin{array}{c}\text { Total fibre } \\
\text { intake }\end{array}$ & Glycaemic index & $\begin{array}{c}\text { Glycaemic } \\
\text { load }\end{array}$ \\
\hline White bread & - & - & - & -0.36 & 0.62 & 0.27 \\
High fibre bread & - & - & - & 0.35 & -0.34 & - \\
Cereal & 0.26 & - & - & 0.40 & - & - \\
Fruit juice & 0.26 & -0.26 & 0.43 & - & - & - \\
Fruit & 0.38 & -0.35 & 0.54 & 0.58 & -0.34 & - \\
Vegetables & - & - & - & 0.51 & - & - \\
Vegetables + fruit & 0.33 & -0.30 & 0.48 & 0.67 & -0.30 & - \\
Added sugar & - & - & 0.28 & - & 0.32 & 0.27 \\
Staple products & - & - & - & - & - & 0.25
\end{tabular}

Food groups and food items included: white bread; high fibre bread (high-fibre white bread, whole meal bread, rye bread, multi-grain bread); cereal (All Bran, bran flakes, muesli, Weet Bix, cornflakes, porridge); fruit (oranges, apples, pears, bananas, melon, pineapple, strawberries, apricots, peaches, mango, avocado, tinned fruit); fruit juice; staple products (rice, pasta); added sugar; vegetables (tomato, tomato sauce, capsicum (bell or sweet peppers), lettuce, cucumber, celery, beetroot, carrots, cabbage, cauliflower, broccoli, spinach, peas, green beans, bean sprouts, pumpkin, onion, garlic, mushrooms, zucchini, potato)

- correlations below 0.25 were considered not relevant and are not displayed

\section{Discussion}

In this large prospective cohort study, we found that carbohydrate quantity assessed with the LCD score was associated with GDM, whereas for carbohydrate quality we observed an inverse association between fibre intake and GDM. Furthermore, higher intakes of cereal were positively associated with GDM risk, whereas higher intakes of fruit and fruit juice were associated with lower risk of GDM. 
Table 5.4: Relative risks of GDM for carbohydrate-rich food groups.

\begin{tabular}{lccccc}
\hline & \multicolumn{4}{c}{ Risk of GDM - per unit increment } \\
\cline { 2 - 6 } & P for linear trend & Unit increment & Model 1 & Model 2 & Model 2+BMI \\
\hline White bread & 0.01 & $50 \mathrm{~g} /$ day & $1.18(1.02-1.36)$ & $1.05(0.86-1.27)$ & $1.05(0.86-1.27)$ \\
High fibre bread & 0.01 & $50 \mathrm{~g} /$ day & $0.82(0.70-0.95)$ & $0.84(0.70-1.01)$ & $0.86(0.71-1.03)$ \\
Cereal & 0.32 & $20 \mathrm{~g} /$ day & $1.03(1.00-1.07)$ & $1.03(1.00-1.07)$ & $1.04(1.01-1.07)$ \\
Fruit juice & 0.01 & $100 \mathrm{~g} /$ day & $0.88(0.79-0.99)$ & $0.89(0.79-0.99)$ & $0.89(0.80-1.00)$ \\
Fruit & 0.01 & $50 \mathrm{~g} /$ day & $0.94(0.89-0.98)$ & $0.94(0.89-0.99)$ & $0.95(0.90-0.99)$ \\
Vegetables & 0.11 & $100 \mathrm{~g} /$ day & $0.94(0.84-1.05)$ & $0.96(0.86-1.07)$ & $0.96(0.86-1.07)$ \\
Vegetables + fruit & 0.01 & $100 \mathrm{~g} /$ day & $0.90(0.83-0.98)$ & $0.90(0.83-0.98)$ & $0.91(0.83-0.99)$ \\
Added sugar & $-\mathrm{a}$ & Users vs. non-users & $1.29(1.01-1.64)$ & $1.22(0.96-1.56)$ & $1.25(0.98-1.59)$ \\
Staple products & 0.45 & $50 \mathrm{~g} /$ day & $0.97(0.89-1.05)$ & $0.96(0.88-1.05)$ & $0.97(0.89-1.06)$ \\
\hline
\end{tabular}

Food groups and food items included: white bread; high fibre bread (high-fibre white bread, whole meal bread, rye bread, multi-grain bread); cereal (All Bran, bran flakes, muesli, Weet Bix, cornflakes, porridge); fruit (oranges, apples, pears, bananas, melon, pineapple, strawberries, apricots, peaches, mango, avocado, tinned fruit); fruit juice; staple products (rice, pasta); added sugar; vegetables (tomato, tomato sauce, capsicum (bell or sweet peppers), lettuce, cucumber, celery, beetroot, carrots, cabbage, cauliflower, broccoli, spinach, peas, green beans, bean sprouts, pumpkin, onion, garlic, mushrooms, zucchini, potato)

Model 1: adjusted for age (years), country of birth (Australia, Asia, or other), educational level (low, medium, or high), total energy intake (kJ/day), physical activity (low, medium, or high), smoking (current, former, or never), polycystic ovarian syndrome (yes or no), hypertension during pregnancy (yes or no), parity $(0,1$, or $\geq 2$ ), inter-pregnancy interval (not applicable [first pregnancy], $<18$ months, 18-60 months, >60 months)

Model 2: model $1+$ additional adjustments for other carbohydrate food groups

Model 2+ BMI: model $2+$ additional adjustments for BMI (normal weight, overweight, or obese)

a Because of the large proportion of non-users, the association between added sugar and risk of GDM was assessed for users ( $n=2154$; median intake $15.5 \mathrm{~g} /$ day) vs. non-users $(n=1453$ ) instead of a linear association

In this study, we examined both pre-pregnancy carbohydrate quantity and quality of the diet. To our knowledge there is only one other prospective cohort study (the Nurses' Health Study) which is comparable to ours and looked at carbohydrate quantity and various aspects of carbohydrate quality (i.e. fibre, glycaemic index and glycaemic load) in relation to GDM incidence. In our study, a higher total carbohydrate intake was inversely associated with GDM, but this association disappeared after adjustment for fat and protein intake. The LCD diet score was significantly and positively associated with GDM risk, thus women with a relative low carbohydrate intake had a higher risk of GDM. In the Nurses' Health Study, women with a high LCD score (e.g. a low carbohydrate intake) also had a higher risk of GDM, with similar effect estimates [13]. Bao et al. were also able to calculate an animal and vegetable LCD score, which indicated that especially women with a high intake of animal fat and protein were at a higher risk. This is further supported by other studies showing higher GDM risk and impaired glucose metabolism with higher intakes of animal fat [32-34] and animal protein [35]. This could indicate that not total carbohydrate intake, but rather protein and fat intake are important in the association with GDM risk. However, the group of carbohydrates is a large group with different 
types of carbohydrates, including complex polysaccharides, mono-and disaccharides and different types of fibre. Some have beneficial health effects, such as fibre and low GI-diets [11, 36, 37], whereas others have negative health effects such as sugars [38]. This could be a reason for the absence of an association of total carbohydrate intake with GDM.

Therefore, we further examined the relationship between carbohydrates and GDM by investigating carbohydrate quality e.g. different types of carbohydrates. We examined fibre and total sugar (i.e. total mono- and disaccharide) intake, glycaemic index, glycaemic load and intake of several carbohydraterich food groups. In our study, dietary fibre, vegetables and fruit, fruit and fruit juice were significantly associated with a lower risk of GDM, whereas cereal intake was associated with a higher risk of GDM. The association between fibre and GDM risk observed in our study is in line with results of the Nurses' Health Study [14]. The Nurses' Health Study adjusted for BMI in all models, whereas in our study adjustment for BMI attenuated the association. However, it should be noted that the magnitude of the association after BMI adjustment was comparable to the association observed in the Nurses' Health Study. Our observation of attenuation by BMI could indicate that the association between fibre and GDM risk is mediated by BMI. One of the underlying mechanisms could be that increased fibre intake reduces appetite and energy intake $[39,40]$. This could lead to reduced adiposity and improved insulin sensitivity $[41,42]$ and thus a lower risk of GDM.

Furthermore, fibre intake was strongly correlated with fruits, vegetables, white bread, high-fibre bread and cereal intake. Of these food groups, the most predominant association was observed between high fruit intake and lower risk of GDM, followed by high cereal intake and higher risk of GDM. An inverse not significant association was observed for high fibre bread and a positive not significant association of white bread with GDM, after adjustment for other food groups. The multitude of directions and magnitude of the associations between different food groups high in fibre and GDM illustrates the complexity of the association between fibre and GDM. The association of higher cereal intake with higher risk of GDM could potentially be explained by the often high amounts of sugar present in cereal products, whereas fruit contains many other nutrients such as vitamins and minerals that could also have a beneficial effect on GDM risk [43]. Furthermore, although we could not differentiate the different types of dietary fibre in our study, this could explain the observed associations. Whole grain products contain mainly insoluble fibre [44], which has been associated with intestinal transit time [45], whereas fruit and vegetables contain relative more soluble fibres [44]. Soluble fibres can create a gel-like substance in the stomach, which can delay gastric emptying and thus slow glucose absorption $[46,47]$. However, confirmation by experimental studies and more detailed knowledge of underlying mechanisms is needed. 
Carbohydrate quality is most often studied by using the GI and GL. We found no statistically significant associations between GI, GL and GDM risk. However, it should be noted that the direction and magnitude of the associations between $\mathrm{Gl}, \mathrm{GL}$ and GDM risk were similar to significant estimates shown in the Nurses' Health Study [14]. Differences in study size and number of cases could explain the absence of statistical significance in our study. Research on GI and GL in pregnancy is limited, but indicates that pregnant women could benefit from low GI and GL diets to lower maternal glycosylated haemoglobin, plasma glucose, birth weight, and reduce insulin requirements of women with GDM [4850]. Furthermore, there is substantial evidence that relates low GI and GL to lower risk of T2DM [11] and that low $\mathrm{Gl}$ and $\mathrm{GL}$ diets may reduce hyperlipidaemia and improve insulin sensitivity $[51,52]$. Overall, this suggests a beneficial effect of low GI and GL on GDM risk.

The potential effect of sugar intake on disease risk can be a controversial topic [38]. In our study, we examined associations between sugar intake and GDM risk by examining several exposures: total sugar intake (all mono- and disaccharides), sugar added by participants to their meals and drinks, and carbohydrate food groups with high sugar content (fruit and fruit juice). Total sugar intake was not associated with GDM in our study, but fruit, fruit juice and added sugar were (borderline significantly) associated with GDM risk. Higher fruit and fruit juice intakes were associated with lower risk of GDM whereas added sugar with a higher risk of GDM. The discrepancy in our results could be due to the complexity of total sugar content. Total sugar includes sugars found in nutritious foods such as fruit, fruit juice and dairy products, whereas on the other hand added sugar provides only excess energy. Our findings, except for those on fruit juice, are in line with dietary recommendations from leading institutes, such as the World Health Organization that recommend a reduction in free sugars [53].

To our knowledge, no other studies investigated intake of carbohydrate-rich food groups and risk of GDM. The observed associations between carbohydrate-rich food groups and GDM risk, i.e. higher risks observed for cereal and added sugar intake, and lower risks for vegetables and fruit, fruit, fruit juice and high-fibre bread, are consistent with results from studies on dietary pattern analyses and risk of GDM $[31,54,55]$. Healthy dietary patterns and diet quality scores (e.g. Mediterranean Diet score, Healthy Eating Index) often include vegetables, fruit and whole grain products and limited intake of refined grains. Healthy dietary patterns are consistently associated with lower risk of GDM. Pinpointing specific food groups underlying the associations between carbohydrates and GDM is important for development of effective prevention strategies, as it might be easier to change intake of specific food groups rather than a complete dietary pattern. However, more studies and specifically randomized clinical trials are needed to confirm our results and to investigate whether changing intakes of specific food groups have an impact on reducing GDM risk. 
The results presented were derived using data from a large, prospective study. Women were included in the study early in their reproductive age and before pregnancy. The longitudinal design with multiple measurements enables the examination of prospective associations with risk of developing GDM. Additionally, we were able to use updated information on covariates such as BMI, educational level, and smoking, which might change over time since the start of the study, especially in this young cohort. Results from this nationally representative population-based sample are generalizable to the Australian population of reproductive-aged women [17] and other Western countries with similar sources of carbohydrate intake. Furthermore, in this study we looked at both quantity and quality of carbohydrates to provide a complete overview.

However, some limitations should also be acknowledged. First, data from this study is observational and no causal effects can be established. Secondly, data is obtained from self-reports and therefore misclassification could be present, although self-reported GDM outcome was validated against medical records [26]. Furthermore, validation of the FFQ showed good agreement with food-records (energyadjusted correlation coefficients of 0.78 for carbohydrate) [20], indicating that most important carbohydrate sources are properly assessed with the FFQ. However, food group intake was not validated and sugar-sweetened beverages were not included. Furthermore, food group analysis was limited by aggregation of foods in the FFQ food items. For example, it was not possible to differentiate between whole-grain pasta and refined grain pasta in the staple group. Also, aggregation of foods in the FFQ food items might have affected the Gl associations, as aggregation of foods with different GI values could have led to misclassification. Thirdly, dietary intake during pregnancy was not assessed in this study. However, a recent study investigating diet quality of women before and during pregnancy in the ALSWH showed that there were few differences in dietary intake between non-pregnant and pregnant women [56], as is also reported by other studies [57, 58]. Finally, although we were able to adjust for a wide variety of socio-demographic and lifestyle factors, residual confounding might still be present, e.g. consumption of certain food items could reflect health consciousness.

\section{Conclusions}

A relatively low carbohydrate and high fat and protein intake may increase risk of GDM; however, it is important to take the source of carbohydrate into account. High intake of total dietary fibre, fruit and fruit juice may decrease risk of GDM, whereas cereal could increase risk of GDM. This may be important to consider in nutritional programs for preventing GDM. 
Acknowledgements: The research on which this paper is based was conducted as part of the Australian Longitudinal Study on Women's Health by the University of Queensland and the University of Newcastle. We are grateful to the Australian Government Department of Health for funding and to the women who provided the survey data.

The authors thank Professor Graham Giles of the Cancer Epidemiology and Intelligence Division of Cancer Council Victoria, for permission to use the Dietary Questionnaire for Epidemiological Studies (Version 2), Melbourne: Cancer Council Victoria, 1996.

The Jo Kolk study fund is gratefully acknowledged for proving $M L$ with a travel grant to visit the University of Queensland. G.D.M is supported by the Australian National Health and Medical Research Council Principal Research Fellowship (APP1121844). 


\section{References}

1. Bardenheier, B.H., G. Imperatore, S.M. Gilboa, et al., Trends in Gestational Diabetes Among Hospital Deliveries in 19 U.S. States, 2000-2010. Am. J. Prev. Med., 2015. 49(1): p. 12-9.

2. Buckley, B.S., J. Harreiter, P. Damm, et al., Gestational diabetes mellitus in Europe: prevalence, current screening practice and barriers to screening. A review. Diabet. Med., 2012. 29(7): p. 844-54.

3. Hapo Study Cooperative Research Group, B.E. Metzger, L.P. Lowe, et al., Hyperglycemia and adverse pregnancy outcomes. N. Engl. J. Med., 2008. 358(19): p. 1991-2002.

4. Bellamy, L., J.P. Casas, A.D. Hingorani, et al., Type 2 diabetes mellitus after gestational diabetes: a systematic review and meta-analysis. Lancet, 2009. 373(9677): p. 1773-9.

5. Boney, C.M., A. Verma, R. Tucker, et al., Metabolic syndrome in childhood: association with birth weight, maternal obesity, and gestational diabetes mellitus. Pediatrics, 2005. 115(3): p. e290-6.

6. Clausen, T.D., E.R. Mathiesen, T. Hansen, et al., High prevalence of type 2 diabetes and pre-diabetes in adult offspring of women with gestational diabetes mellitus or type 1 diabetes: the role of intrauterine hyperglycemia. Diabetes Care, 2008. 31(2): p. 340-6.

7. Schoenaker, D.A., G.D. Mishra, L.K. Callaway, et al., The Role of Energy, Nutrients, Foods, and Dietary Patterns in the Development of Gestational Diabetes Mellitus: A Systematic Review of Observational Studies. Diabetes Care, 2016. 39(1): p. 16-23.

8. Zhang, C. and Y. Ning, Effect of dietary and lifestyle factors on the risk of gestational diabetes: review of epidemiologic evidence. Am. J. Clin. Nutr., 2011. 94(6 Suppl): p. 1975S-1979S.

9. Catalano, P.M., L. Huston, S.B. Amini, et al., Longitudinal changes in glucose metabolism during pregnancy in obese women with normal glucose tolerance and gestational diabetes mellitus. Am. J. Obstet. Gynecol., 1999. 180(4): p. 903-16.

10. Heer, M. and S. Egert, Nutrients other than carbohydrates: their effects on glucose homeostasis in humans. Diabetes Metab. Res. Rev., 2015. 31(1): p. 14-35.

11. Greenwood, D.C., D.E. Threapleton, C.E. Evans, et al., Glycemic index, glycemic load, carbohydrates, and type 2 diabetes: systematic review and dose-response meta-analysis of prospective studies. Diabetes Care, 2013. 36(12): p. 4166-71.

12. Schulze, M.B., S. Liu, E.B. Rimm, et al., Glycemic index, glycemic load, and dietary fiber intake and incidence of type 2 diabetes in younger and middle-aged women. Am. J. Clin. Nutr., 2004. 80(2): p. 348-56.

13. Bao, W., K. Bowers, D.K. Tobias, et al., Prepregnancy low-carbohydrate dietary pattern and risk of gestational diabetes mellitus: a prospective cohort study. Am. J. Clin. Nutr., 2014.

14. Zhang, C., S. Liu, C.G. Solomon, et al., Dietary fiber intake, dietary glycemic load, and the risk for gestational diabetes mellitus. Diabetes Care, 2006. 29(10): p. 2223-30.

15. Russell, W.R., A. Baka, I. Bjorck, et al., Impact of Diet Composition on Blood Glucose Regulation. Crit. Rev. Food Sci. Nutr., 2016. 56(4): p. 541-90.

16. Halton, T.L., W.C. Willett, S. Liu, et al., Low-carbohydrate-diet score and the risk of coronary heart disease in women. N. Engl. J. Med., 2006. 355(19): p. 1991-2002.

17. Brown, W.J., L. Bryson, J.E. Byles, et al., Women's Health Australia: recruitment for a national longitudinal cohort study. Women Health, 1998. 28(1): p. 23-40.

18. Lee, C., A.J. Dobson, W.J. Brown, et al., Cohort Profile: the Australian Longitudinal Study on Women's Health. Int. J. Epidemiol., 2005. 34(5): p. 987-91.

19. Huang, T.T., S.B. Roberts, N.C. Howarth, et al., Effect of screening out implausible energy intake reports on relationships between diet and BMI. Obes. Res., 2005. 13(7): p. 1205-17.

20. Hodge, A., A.J. Patterson, W.J. Brown, et al., The Anti Cancer Council of Victoria FFQ: relative validity of nutrient intakes compared with weighed food records in young to middle-aged women in a study of iron supplementation. Aust. N. Z. J. Public Health, 2000. 24(6): p. 576-83.

21. Ireland, P., D. Jolley, G. Giles, et al., Development of the Melbourne FFQ: a food frequency questionnaire for use in an Australian prospective study involving an ethnically diverse cohort. Asia Pac. J. Clin. Nutr., 1994. 3(1): p. $19-31$.

22. Lewis, J., G. Milligan, and A. Hunt, Nuttab95: Nutrient data table for use in Australia. 1995, Australian Government Publishing Service: Canberra.

23. Wolever, T.M., D.J. Jenkins, A.L. Jenkins, et al., The glycemic index: methodology and clinical implications. Am. J. Clin. Nutr., 1991. 54(5): p. 846-54.

24. Foster-Powell, K., S.H. Holt, and J.C. Brand-Miller, International table of glycemic index and glycemic load values: 2002. Am. J. Clin. Nutr., 2002. 76(1): p. 5-56.

25. Willett, W.C., G.R. Howe, and L.H. Kushi, Adjustment for total energy intake in epidemiologic studies. Am. J. Clin. Nutr., 1997. 65(4 Suppl): p. 1220S-1228S; discussion 1229S-1231S.

26. Gresham, E., P. Forder, C.L. Chojenta, et al., Agreement between self-reported perinatal outcomes and administrative data in New South Wales, Australia. BMC Pregnancy Childbirth, 2015. 15: p. 161.

27. Brown, W.J., N.W. Burton, A.L. Marshall, et al., Reliability and validity of a modified self-administered version of the Active Australia physical activity survey in a sample of mid-age women. Aust. N. Z. J. Public Health, 2008. 32(6): p. 535-41. 
28. Hanley, J.A., A. Negassa, M.D. Edwardes, et al., Statistical analysis of correlated data using generalized estimating equations: an orientation. Am. J. Epidemiol., 2003. 157(4): p. 364-75.

29. Spiegelman, D. and E. Hertzmark, Easy SAS calculations for risk or prevalence ratios and differences. Am. J. Epidemiol., 2005. 162(3): p. 199-200.

30. Berglund, P., An introduction to multiple imputation of complex sample data using SAS v9.2, in SAS Global Forum Proceedings. 2010, SAS Institute Inc.: Cary, NC.

31. Schoenaker, D.A., S.S. Soedamah-Muthu, L.K. Callaway, et al., Pre-pregnancy dietary patterns and risk of gestational diabetes mellitus: results from an Australian population-based prospective cohort study. Diabetologia, 2015. 58(12): p. 2726-35.

32. Bowers, K., D.K. Tobias, E. Yeung, et al., A prospective study of prepregnancy dietary fat intake and risk of gestational diabetes. Am. J. Clin. Nutr., 2012. 95(2): p. 446-53.

33. Feskens, E.J., S.M. Virtanen, L. Rasanen, et al., Dietary factors determining diabetes and impaired glucose tolerance. A 20-year follow-up of the Finnish and Dutch cohorts of the Seven Countries Study. Diabetes Care, 1995. 18(8): p. 1104-12.

34. Lichtenstein, A.H. and U.S. Schwab, Relationship of dietary fat to glucose metabolism. Atherosclerosis, 2000. 150(2): p. 227-43.

35. Bao, W., K. Bowers, D.K. Tobias, et al., Prepregnancy dietary protein intake, major dietary protein sources, and the risk of gestational diabetes mellitus: a prospective cohort study. Diabetes Care, 2013. 36(7): p. 2001-8.

36. Silva, F.M., C.K. Kramer, J.C. de Almeida, et al., Fiber intake and glycemic control in patients with type 2 diabetes mellitus: a systematic review with meta-analysis of randomized controlled trials. Nutr. Rev., 2013. 71(12): p. 790801.

37. Yao, B., H. Fang, W. Xu, et al., Dietary fiber intake and risk of type 2 diabetes: a dose-response analysis of prospective studies. Eur. J. Epidemiol., 2014. 29(2): p. 79-88.

38. Khan, T.A. and J.L. Sievenpiper, Controversies about sugars: results from systematic reviews and meta-analyses on obesity, cardiometabolic disease and diabetes. Eur. J. Nutr., 2016. 55(Suppl 2): p. 25-43.

39. Kristensen, M. and M.G. Jensen, Dietary fibres in the regulation of appetite and food intake. Importance of viscosity. Appetite, 2011. 56(1): p. 65-70.

40. Wanders, A.J., J.J. van den Borne, C. de Graaf, et al., Effects of dietary fibre on subjective appetite, energy intake and body weight: a systematic review of randomized controlled trials. Obes. Rev., 2011. 12(9): p. 724-39.

41. Ylonen, K., C. Saloranta, C. Kronberg-Kippila, et al., Associations of dietary fiber with glucose metabolism in nondiabetic relatives of subjects with type 2 diabetes: the Botnia Dietary Study. Diabetes Care, 2003. 26(7): p. 197985.

42. Thielecke, F. and S.S. Jonnalagadda, Can whole grain help in weight management? J. Clin. Gastroenterol., 2014. 48 Suppl 1: p. S70-7.

43. Zhang, C., M.A. Williams, T.K. Sorensen, et al., Maternal plasma ascorbic Acid (vitamin C) and risk of gestational diabetes mellitus. Epidemiology, 2004. 15(5): p. 597-604.

44. Lairon, D., S. Bertrais, S. Vincent, et al., Dietary fibre intake and clinical indices in the French Supplementation en Vitamines et Mineraux AntioXydants (SU.VI.MAX) adult cohort. Proc. Nutr. Soc., 2003. 62(1): p. 11-5.

45. Roehrig, K.L., The physiological effects of dietary fiber-a review. Food Hydrocolloid, 1988. 2(1): p. 1-18.

46. McIntosh, M. and C. Miller, A diet containing food rich in soluble and insoluble fiber improves glycemic control and reduces hyperlipidemia among patients with type 2 diabetes mellitus. Nutr. Rev., 2001. 59(2): p. 52-5.

47. Poutanen, K.S., P. Dussort, A. Erkner, et al., A review of the characteristics of dietary fibers relevant to appetite and energy intake outcomes in human intervention trials. Am. J. Clin. Nutr., 2017. 106(3): p. 747-754.

48. Louie, J.C., J.C. Brand-Miller, and R.G. Moses, Carbohydrates, glycemic index, and pregnancy outcomes in gestational diabetes. Curr. Diab. Rep., 2013. 13(1): p. 6-11.

49. Ma, W.J., Z.H. Huang, B.X. Huang, et al., Intensive low-glycaemic-load dietary intervention for the management of glycaemia and serum lipids among women with gestational diabetes: a randomized control trial. Public Health Nutr., 2015. 18(8): p. 1506-13.

50. Scholl, T.O., X. Chen, C.S. Khoo, et al., The dietary glycemic index during pregnancy: influence on infant birth weight, fetal growth, and biomarkers of carbohydrate metabolism. Am. J. Epidemiol., 2004. 159(5): p. 467-74.

51. Dickinson, S. and J. Brand-Miller, Glycemic index, postprandial glycemia and cardiovascular disease. Curr. Opin. Lipidol., 2005. 16(1): p. 69-75.

52. Wolever, T.M., Carbohydrate and the regulation of blood glucose and metabolism. Nutr. Rev., 2003. 61(5 Pt 2): p. S40-8.

53. World Health Organization, Guideline: Sugars intake for adults and children. 2015, World Health Organization: Geneva.

54. Tobias, D.K., C. Zhang, J. Chavarro, et al., Prepregnancy adherence to dietary patterns and lower risk of gestational diabetes mellitus. Am. J. Clin. Nutr., 2012. 96(2): p. 289-95.

55. Tryggvadottir, E.A., H. Medek, B.E. Birgisdottir, et al., Association between healthy maternal dietary pattern and risk for gestational diabetes mellitus. Eur. J. Clin. Nutr., 2016. 70(2): p. 237-42.

56. Gresham, E., C.E. Collins, G.D. Mishra, et al., Diet quality before or during pregnancy and the relationship with pregnancy and birth outcomes: the Australian Longitudinal Study on Women's Health. Public Health Nutr., 2016. 19(16): p. 2975-2983. 


\section{Chapter 5}

57. Cuco, G., J. Fernandez-Ballart, J. Sala, et al., Dietary patterns and associated lifestyles in preconception, pregnancy and postpartum. Eur. J. Clin. Nutr., 2006. 60(3): p. 364-71.

58. McGowan, C.A. and F.M. McAuliffe, Maternal dietary patterns and associated nutrient intakes during each trimester of pregnancy. Public Health Nutr., 2013. 16(1): p. 97-107. 
Carbohydrate intake and GDM risk 



\section{Chapter 6}

Pre-pregnancy dietary micronutrient adequacy and risk of developing gestational diabetes: the Australian Longitudinal

Study on Women's Health

Moniek Looman, Daniëlle A.J.M. Schoenaker, Sabita S. Soedamah-Muthu, Gita D. Mishra, Anouk Geelen, Edith J.M. Feskens 


\section{Abstract}

Aims: The objective of this study was to examine the prevalence of inadequate micronutrient intake before pregnancy and the association between pre-pregnancy dietary micronutrient adequacy, i.e. meeting micronutrient intake recommendations for a range of micronutrients, and risk of developing gestational diabetes (GDM) in an Australian population.

Methods: In the prospective cohort Australian Longitudinal Study on Women's Health, 3,607 women who were aged 25-30 years at baseline in 2003 and had no diabetes were followed-up until 2015. Diet was assessed with a validated 101-item food frequency questionnaire. Micronutrient intake was compared with Australian nutrient reference values. The Micronutrient Adequacy Ratio (MAR) was calculated as the micronutrient intake divided by its recommended dietary intake averaged over thirteen micronutrients. GDM diagnosis was self-reported and validated in a subsample. Multivariable regression models with generalized estimating equations were used to estimate relative risks (RR) and $95 \% \mathrm{Cl}$.

Results: In 6,263 pregnancies, 285 cases of GDM were documented (4.6\%). High prevalences of inadequate dietary micronutrient intake were observed for calcium $(47.9 \%)$, folate $(80.8 \%)$, magnesium (52.5\%), potassium (63.8\%) and vitamin E (78.6\%), indicating suboptimal pre-pregnancy micronutrient intakes. Inadequate intakes of individual micronutrients were not associated with risk of developing GDM. However, women in the highest quartile of the MAR had a 39\% lower risk of developing GDM compared to women in the lowest quartile (RR 0.61, 95\% $\mathrm{Cl} 0.44-0.86$, p for trend $0.01)$.

Conclusions: These results highlight the importance of an adequate pre-pregnancy intake for micronutrients. Further prospective studies are needed to confirm these findings. 


\section{Introduction}

Adequate dietary micronutrient intake before and during pregnancy is essential for optimal growth and development of the foetus [1]. Micronutrients are involved in a vast array of physiological processes such as enzyme activity, signal transduction and transcription pathways, biological functions and oxidative stress [2]. The most well-known example of the importance of adequate micronutrient intake started before conception and continued during pregnancy is the higher risk of neural tube defects due to folate deficiency [3].

Gestational diabetes mellitus (GDM) is one of the most common metabolic complications during pregnancy and prevalence has continued to increase worldwide $[4,5]$. During normal pregnancy, the demand for insulin is increased due to progressive insulin resistance to ensure adequate foetal growth and development. If these insulin requirements are not met, women develop GDM characterized by exaggerated insulin resistance as well as impaired insulin secretion [6]. Few modifiable risk factors for GDM have been identified, but diet has been indicated as one of the most important ones as it is relatively easy to modify $[7,8]$. Recent reviews have summarized evidence that show there is a relation between diet and the development of glucose intolerance in non-pregnant populations [9-11]. Both protective and risk-enhancing associations were observed between different dietary factors and glucose intolerance. Micronutrients act via multiple pathways in the glucose homeostasis [10]. For example, zinc is involved in insulin assembly, thiamine is an essential coenzyme, magnesium is involved in glucose transport, whereas vitamin $\mathrm{E}$ and $\mathrm{C}$ may mitigate metabolic stress, promoting glucose and fatty acid utilization [11]. Thus, micronutrients can play an important role in the complex system of glucose homeostasis.

A limited number of studies have investigated the role of micronutrients in the development of GDM and these studies focussed on specific individual micronutrients [12-16]. A higher consumption of heme iron before and during pregnancy was associated with a higher risk of GDM [13, 14], whereas a higher consumption and plasma concentration of vitamin $C$ and zinc during pregnancy were associated with a lower risk of GDM $[12,15,16]$. However, evidence on pre-pregnancy dietary micronutrient intake in relation to GDM is limited. Furthermore, as micronutrients may have synergistic or antagonistic effect, it is important to look at combined dietary micronutrient intake rather than at intakes of individual micronutrients. To our knowledge, no other studies investigated overall micronutrient adequacy and developing GDM. Therefore, we investigated dietary micronutrient adequacy, which was defined as dietary intake of 13 micronutrients relative to the recommended intake of each micronutrient, and overall dietary micronutrient adequacy before pregnancy using the Micronutrient Adequacy Ratio (MAR). The objective of this study was to examine the prevalence of 
inadequate micronutrient intake before pregnancy and the association between pre-pregnancy dietary micronutrient adequacy and risk of developing GDM in an Australian population.

\section{Methods}

\section{Study design and population}

The current study used data from the young cohort of the Australian Longitudinal Study on Women's Health (ALSWH). ALSWH is an ongoing population-based prospective cohort study investigating the role of demographic, social, physical, psychological, and behavioural factors in women's health. The study design, recruitment, methods and responses have been described elsewhere $[17,18]$. Briefly, in 1996 approximately 15,000 women born in 1973-78 (18-23 years) were recruited. Women were randomly selected from Australia's nationalized health-care system, Medicare, with intentional oversampling in rural and remote areas. Self-administered questionnaires were sent to participants every 3-4 years. Dietary intake was first collected in $2003(n=9,081)$ when women were 25-30 years, and this time point was therefore used as baseline for the present analyses. Informed consent was obtained from all participants at each survey and the study was approved by the Human Research Ethics Committees of the Universities of Newcastle and Queensland.

In Figure 6.1, a flowchart for detailed breakdown of the sample size for this project is displayed. Women were excluded from the current analyses if they did not report a live birth at follow-up surveys in $2006,2009,2012$ or 2015 , were pregnant at the baseline survey, had missing data on diet at the baseline survey (2003) or follow-up survey (2009), had missing data on GDM, reported implausible energy intake (ratio of reported energy intake and predicted energy requirement $<0.56$ or $>1.44$ [19]), had a history of type 1 or type 2 diabetes mellitus prior to GDM diagnosis, had a history of GDM prior to baseline, or had missing covariate data. In total 3,607 women who experienced a total of 6,263 pregnancies were included in the analyses.

\section{Dietary assessment}

Dietary intake was assessed using the Dietary Questionnaire for Epidemiological Studies (DQES) FFQ version 2. This 101-item FFQ assesses usual food and beverage intake of the previous 12 months. Information on frequency and dose of vitamin and/or mineral supplementation was not included in the FFQ. The development and evaluation of this FFQ has been described elsewhere [20,21]. Briefly, participants were asked to report their usual frequency of consumption of 74 food items and six alcoholic beverage items using a 10-point scale ranging from 'Never' to 'Three or more times per day'. Portion size photographs were used to adjust the serving sizes. Twenty-one items were 


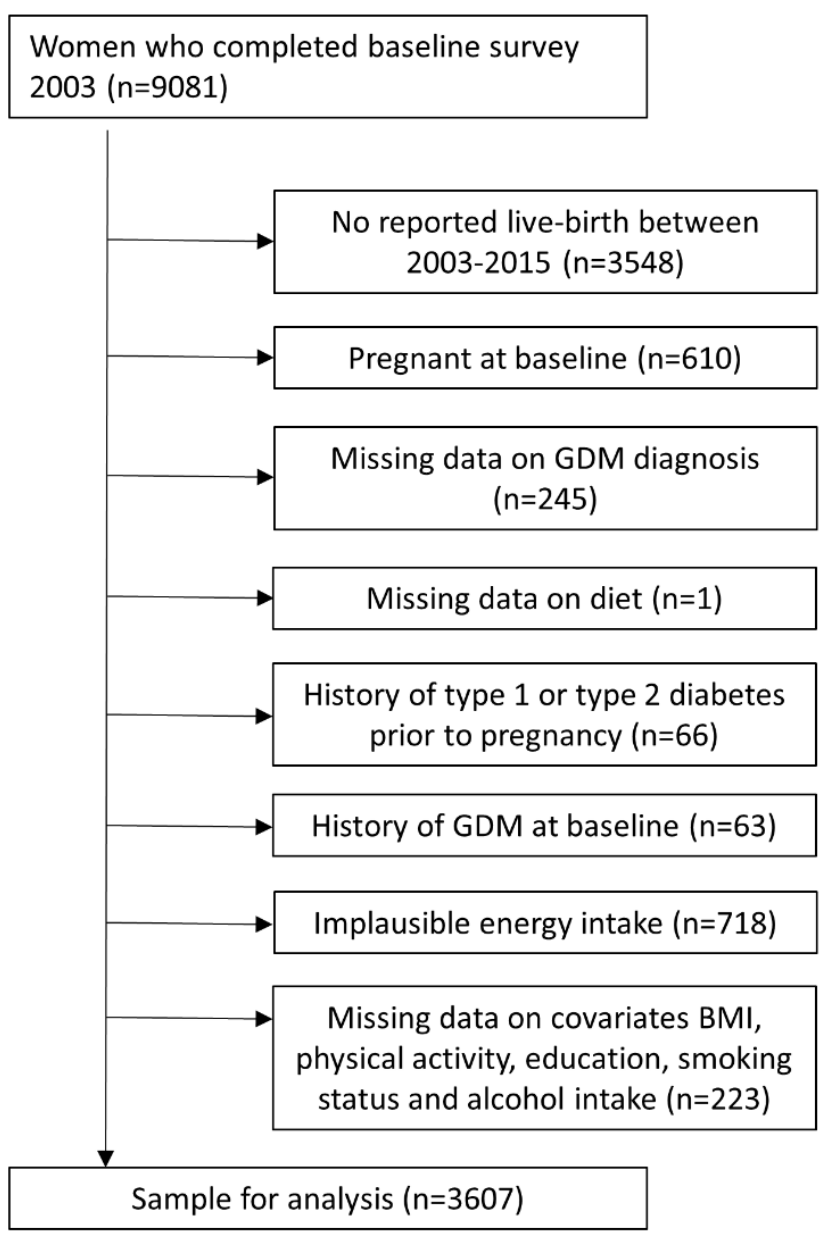

Figure 6.1: Flow chart of the study population.

included on the number of servings of milk, bread, sugar and eggs, and the type of milk, bread, fat spread and cheese consumed. Nutrient intakes were computed using the national government food composition database of Australian foods, the NUTTAB95 [22]. Available micronutrient intakes in this study were: vitamin A, folate, niacin, riboflavin, thiamine, vitamin C, vitamin $E$, calcium, iron, potassium, zinc, phosphorus and magnesium. Validation of the FFQ against 7 day food diaries of 63 women of reproductive age showed moderate to strong energy-adjusted correlation coefficients for a wide range of macro- and micronutrients (ranging from 0.28 for vitamin A to 0.69 for magnesium) [20]. Information on dietary intake was collected at baseline (2003) and during a follow-up survey in 2009. As dietary intake can change over time the most recent reported dietary intake before the pregnancy was used. 


\section{Micronutrient adequacy}

Nutrient Reference Values for Australia and New Zealand, published in 2005 by the National Health and Medical Research Council of Australia, were used to assess adequacy and inadequate micronutrient intakes [23]. The definitions of the Australian Nutrient Reference Values used in this study can be found in Table 6.1. The Estimated Average Requirement (EAR) cut point method was used to assess the prevalence of inadequate micronutrient intake on a population level, by assessing the proportion of the population below the EAR [24]. No EAR was available for vitamin E and potassium, therefore, the Adequate Intake (AI) was used as an alternative to assess the prevalence of inadequate micronutrient intake on a population level.

To assess micronutrient adequacy for individuals, the Nutrient Adequacy Ratio (NAR) was calculated $[25,26]$. The NAR is a measure of an individual's micronutrient adequacy, by comparing the individual's daily intake of a nutrient with the RDI for that nutrient. A NAR ranges between 0 and 1.0. A NAR of 1.0 indicates that intake of that nutrient equals the RDI, whereas a value below 1.0 indicates an intake lower than the RDI (i.e. inadequacy). The Mean Adequacy Ratio (MAR) is calculated as the average of the NAR values for the selected nutrients for a certain individual $[25,26]$. The MAR is derived by summing the NARs and dividing by the number of micronutrients assessed. The MAR is thus a summary measure of micronutrient adequacy with a MAR of 1.0 indicating that for all 13 micronutrients intake is equal or higher than recommended. As micronutrient intake was highly correlated with total energy intake $(r 0.50-0.81)$, the nutrient residual method was used to adjust for energy intake [27].

Table 6.1: Definitions and abbreviations of the nutrient reference values used in the current study.

\begin{tabular}{|c|c|c|c|}
\hline Nutrient Reference Value & Abbreviation & Definition & Level \\
\hline $\begin{array}{l}\text { Estimated Average } \\
\text { Requirement }^{\mathrm{a}}\end{array}$ & EAR & $\begin{array}{l}\text { Daily nutrient intake level needed to meet the requirements } \\
\text { of half the healthy individuals in a particular life stage and } \\
\text { gender group }\end{array}$ & Population \\
\hline Adequate Intake ${ }^{b}$ & Al & $\begin{array}{l}\text { Average daily nutrient intake level based on observed or } \\
\text { experimentally determined approximations or estimates of } \\
\text { nutrient intakes by a group (or groups) of apparently } \\
\text { healthy people that are assumed to be adequate }\end{array}$ & Population \\
\hline Recommended Dietary Intake & RDI & $\begin{array}{l}\text { The average daily dietary intake level that is sufficient to } \\
\text { meet the nutrient requirements of nearly all (97-98 per } \\
\text { cent) healthy individuals in a particular life stage and gender } \\
\text { group }\end{array}$ & Individual \\
\hline
\end{tabular}

a EAR was available for vitamin A, folate, niacin, riboflavin, thiamine, vitamin C, calcium, iron, zinc, phosphorus, magnesium.

${ }^{b}$ Al was used for vitamin E and potassium. 


\section{Assessment of GDM}

Diagnosis of GDM was assessed at each survey and for each live birth using the following question: 'Were you diagnosed by a doctor or treated for gestational diabetes?'. During the study period, diagnostic criteria for GDM in Australia included a 1-hour venous plasma glucose level $\geq 6.55 \%$ (7.8 $\mathrm{mmol} / \mathrm{l})$ after a 50g glucose load; or a 1-hour venous plasma glucose level $\geq 6.65 \%$ ( $8.0 \mathrm{mmol} / \mathrm{l})$ after a $75 \mathrm{~g}$ glucose load. Diagnosis was confirmed with a $75 \mathrm{~g}$ oral glucose tolerance test (fasting) with a venous plasma glucose level at 0 -hours of $\geq 5.1 \%$ ( $5.6 \mathrm{mmol} / \mathrm{l})$ and/or at 2 -hours of $\geq 6.65 \%(8.0 \mathrm{mmol} / \mathrm{l})$ [28]. A reliability study among a subgroup of women from New South Wales ( $n=1,914$ ) has demonstrated high agreement of $91 \%$ between self-reported GDM diagnosis in the study and administrative data records [29].

\section{Covariates}

Self-reported information on country of birth was reported at the first questionnaire at the start of the cohort study. Information on highest qualification completed, number of hours paid work, marital status, parity, hypertensive disorders of pregnancy, polycystic ovary syndrome, inter-pregnancy interval, smoking, physical activity and body mass index (BMI) was self-reported at each survey round (2003, 2006, 2009, 2012 and 2015). Physical activity was assessed using validated questions on frequency and duration of walking and on moderate- and vigorous-intensity activity and was categorized as sedentary/low ( $<600$ total metabolic equivalent [MET] min/week), moderate (600 to $<1200 \mathrm{MET} \mathrm{min} /$ week) or high ( $\geq 1200 \mathrm{MET} \mathrm{min} /$ week) [30]. BMI was categorized as underweight (BMI $<18.5 \mathrm{~kg} / \mathrm{m}^{2}$ ), normal weight (BMI 18.5 to $<25 \mathrm{~kg} / \mathrm{m}^{2}$ ), overweight (BMI 25 to $<30 \mathrm{~kg} / \mathrm{m}^{2}$ ) or obese $\left(B M I \geq 30 \mathrm{~kg} / \mathrm{m}^{2}\right)$. Only a few women were classified as underweight $(n=123,3.4 \%)$; therefore, the underweight and normal weight groups were combined as normal weight $\left(\mathrm{BMI}<25 \mathrm{~kg} / \mathrm{m}^{2}\right)$.

\section{Statistical analysis}

Participants' characteristics reported at baseline were compared across the four quartiles of the MAR score using ANOVA and $\chi^{2}$ tests. Characteristics were weighted by area of residence to account for oversampling of women from rural and remote areas.

Generalized estimating equations (GEE) analyses were used to account for correlated observations due to multiple pregnancies by the same participant [31]. As log-binomial models did not converge, log-Poisson models were used to estimate relative risks (RR) and $95 \%$ confidence intervals $(95 \% \mathrm{Cl})$ [32] for associations between inadequate micronutrient intakes, MAR and development of GDM. Model 1 was adjusted for age at pregnancy, country of birth, educational level, vitamin and mineral supplement use, smoking, physical activity, energy intake, PCOS, hypertension during pregnancy, inter-pregnancy interval, and parity. Model 2 was additionally adjusted for carbohydrate, protein, 
saturated fat, and fibre intake. Model 3 was additionally adjusted for BMI. Adjustment for time-varying covariates (age at pregnancy, education level, BMI, vitamin and mineral supplement use, smoking, physical activity, parity, PCOS, dietary factors) was performed using the value reported the survey administered prior to the pregnancy. For pregnancy-specific covariates (hypertension during pregnancy and, if applicable, inter-pregnancy interval) the value reported for that specific pregnancy was used. Multiple gestation, alcohol intake, area of residence, work status and marital status were not included in the analyses, as these were not significant confounders based on the data. Smoking, vitamin and mineral supplement use and physical activity were also not significant confounders based on the data, but were kept in the model.

Additional analyses were conducted to investigate effect modification by BMI, parity and education level, as these are known risk factors for GDM and have been reported as possible effect modifiers [33-35]. Effect modification was investigated by adding a cross-product interaction term to the maineffects multivariable model and by stratification.

To examine the robustness of the associations observed we performed several sensitivity analyses. First, we averaged dietary intake data from the baseline survey in 2003 and follow-up survey in 2009 to estimate long-term average dietary intake $(n=2,613)$. Furthermore, to exclude possible misclassification due to women changing their normal diet to increase chance of conception, all pregnancies within the first two years of follow-up $(n=864)$ were excluded. Additionally, we conducted a multiple imputation analysis to assess the influence of participant exclusions that resulted from missing covariate data (BMI, physical activity, educational level, smoking status, and alcohol intake; $\mathrm{n}=223$ ) using SAS procedures MI and MIANALYZE [36].

Statistical analyses were conducted using SAS Software Version 9.4 (SAS Institute Inc., Cary, NC, USA). A p value $<0.05$ was considered statistically significant.

\section{Results}

During 12 years of follow-up (2003-2015), 285 cases of GDM (4.6\%) were reported among 3,607 women with 6,263 pregnancies. Women with a MAR in the lowest quartile were younger when they were pregnant, more likely to live in an urban area, be born in Asia, have a lower educational level, be less physically active, be a current smoker, use vitamin and mineral supplements less often, and be multiparous compared to women in the highest quartile (Table 6.2). Although energy intake significantly differed between the four quartiles, no clear trend was observed. Women with a MAR in 
the highest quartile had lower intakes of fat and saturated fat and higher intakes of protein, carbohydrates, and fibre than women in the lowest quartile. In Supplementary Table 6.1, median micronutrient intakes for the MAR quartiles are provided.

In Table 6.3, median micronutrient intakes and prevalence of inadequate micronutrient intakes are shown for women who developed GDM and those who did not. Vitamin C intake was lower in women who developed GDM (99 mg (interquartile range [IQR] $64 \mathrm{mg}$ ) vs. $109 \mathrm{mg}$ (IQR $73 \mathrm{mg}$ ), p=0.002)), whereas micronutrient intakes of zinc and phosphorus were higher $(p<0.05)$ in women who developed GDM compared to those without GDM (Table 3). Prevalence of inadequate micronutrient intakes, based on the EAR-cut point method, ranged from $80.9 \%$ for folate to $0 \%$ for niacin, vitamin $\mathrm{C}$ and phosphorus. High prevalence of inadequate dietary micronutrient intake was observed for calcium (47.9\%), folate (80.8\%), magnesium (52.5\%), potassium (63.8\%) and vitamin E (78.6\%). Prevalence of inadequate intakes for individual micronutrients did not differ between women who developed GDM and those without, and inadequate intake of a single micronutrient was not associated with a higher or lower risk of developing GDM after adjustment for covariates (Table 6.3).

Table 6.2: Baseline characteristics of 3,607 non-pregnant Australian women according to quartile of mean adequacy ratio (MAR).

\begin{tabular}{|c|c|c|c|c|c|}
\hline \multirow[b]{3}{*}{ Characteristics $^{a}$} & \multicolumn{4}{|c|}{ Quartiles of mean adequacy ratio (MAR) } & \multirow[b]{3}{*}{$p$-value } \\
\hline & Quartile 1 & Quartile 2 & Quartile 3 & Quartile 4 & \\
\hline & $\mathrm{N}=901$ & $N=899$ & $\mathrm{~N}=904$ & $\mathrm{~N}=903$ & \\
\hline Median MAR & 0.81 & 0.87 & 0.90 & 0.95 & \\
\hline Age at baseline (yrs) & $27.5(1.5)$ & $27.6(1.5)$ & $27.5(1.4)$ & $27.5(1.5)$ & 0.72 \\
\hline Age at pregnancy (yrs) & $30.3(3.2)$ & $30.4(3.0)$ & $30.9(3.2)$ & $31.1(4.1)$ & $<0.001$ \\
\hline Area of residence & & & & & $<0.001$ \\
\hline Urban & 78.3 & 71.3 & 70.3 & 74.3 & \\
\hline Rural/remote & 21.7 & 28.7 & 29.7 & 25.7 & \\
\hline Country of birth & & & & & $<0.001$ \\
\hline Australia & 88.4 & 91.6 & 92.8 & 92.2 & \\
\hline Asia & 4.5 & 0.9 & 0.9 & 0.6 & \\
\hline Other & 7.1 & 7.5 & 6.3 & 7.2 & \\
\hline Highest educational level & & & & & $<0.001$ \\
\hline Up to year 12 or equivalent & 22.0 & 20.5 & 18.5 & 14.0 & \\
\hline Trade/apprenticeship/certificate/diploma & 25.4 & 23.4 & 19.5 & 18.1 & \\
\hline University/higher degree & 52.6 & 56.1 & 62.0 & 67.9 & \\
\hline Work status & & & & & 0.13 \\
\hline No-paid job & 15.6 & 15.4 & 15.4 & 14.5 & \\
\hline Part-time & 19.7 & 22.1 & 24.4 & 19.6 & \\
\hline Full-time & 64.7 & 62.5 & 60.3 & 65.9 & \\
\hline Marital status & & & & & 0.002 \\
\hline Married/in a relationship & 64.0 & 71.0 & 64.7 & 66.2 & \\
\hline Separated/divorced/widowed & 3.8 & 2.9 & 1.9 & 2.0 & \\
\hline $\mathrm{BMI}\left(\mathrm{kg} / \mathrm{m}^{2}\right)$ & $23.7(4.8)$ & $24.0(4.6)$ & $23.9(4.6)$ & $23.4(4.1)$ & 0.01 \\
\hline
\end{tabular}


Table 6.2 continued:

\begin{tabular}{|c|c|c|c|c|c|}
\hline BMI & & & & & 0.02 \\
\hline Healthy weight $\left(<25 \mathrm{~kg} / \mathrm{m}^{2}\right)$ & 72.4 & 69.3 & 69.3 & 75.1 & \\
\hline Overweight $\left(25\right.$ to $<30 \mathrm{~kg} / \mathrm{m}^{2}$ ) & 17.6 & 19.2 & 20.6 & 18.1 & \\
\hline Obese $\left(\geq 30 \mathrm{~kg} / \mathrm{m}^{2}\right)$ & 10.0 & 11.5 & 10.1 & 6.9 & \\
\hline Physical activity & & & & & $<0.001$ \\
\hline Sedentary/low (<600 MET min/week) & 48.5 & 46.5 & 37.7 & 31.9 & \\
\hline Moderate (600 to <1200 MET min/week) & 23.4 & 23.8 & 27.5 & 25.7 & \\
\hline High ( $\geq 1200 \mathrm{MET}$ min/week) & 28.1 & 30.7 & 34.5 & 42.3 & \\
\hline Smoking status & & & & & $<0.001$ \\
\hline Never smoked & 58.8 & 62.0 & 32.3 & 66.2 & \\
\hline History of smoking & 17.2 & 15.7 & 18.3 & 19.1 & \\
\hline Current smoker & 24.0 & 22.3 & 19.4 & 14.7 & \\
\hline Alcohol intake status & & & & & 0.17 \\
\hline Non drinker & 5.4 & 5.6 & 5.1 & 4.6 & \\
\hline Low risk/rarely drinks & 90.4 & 90.1 & 92.5 & 92.6 & \\
\hline High risk/often drinks & 4.2 & 4.3 & 2.4 & 2.8 & \\
\hline Vitamin and mineral supplement use & & & & & 0.04 \\
\hline Never/rarely & 36.9 & 34.2 & 33.2 & 30.3 & \\
\hline Sometimes & 25.7 & 23.5 & 24.7 & 25.3 & \\
\hline Often & 37.4 & 42.3 & 42.1 & 44.4 & \\
\hline Nulliparous & 75.9 & 76.1 & 77.7 & 84.2 & $<0.001$ \\
\hline Polycystic ovary syndrome & 9.3 & 8.2 & 8.1 & 8.9 & 0.75 \\
\hline Total energy intake (kJ/day) & $6975(2197)$ & $7190(1711)$ & $7179(1526)$ & $6892(1263)$ & $<0.001$ \\
\hline Total fat intake (E\%) & $38.3(5.4)$ & $37.6(5.0)$ & $35.7(4.9)$ & $33.4(5.2)$ & $<0.001$ \\
\hline Total saturated fat intake (E\%) & $16.3(3.2)$ & $15.7(3.1)$ & $14.6(2.8)$ & $13.2(2.9)$ & $<0.001$ \\
\hline Total protein intake (E\%) & $19.4(3.3)$ & $19.9(3.1)$ & $20.1(3.0)$ & $20.5(3.1)$ & $<0.001$ \\
\hline Total carbohydrate intake (E\%) & $42.7(6.4)$ & $42.8(5.7)$ & $44.5(5.4)$ & $46.2(5.9)$ & $<0.001$ \\
\hline Total fibre intake (g/day) & $16.1(6.1)$ & $18.6(5.7)$ & $20.7(5.6)$ & $23.6(5.4)$ & $<0.001$ \\
\hline
\end{tabular}

Values are mean (SD) or \%

a Baseline characteristics (2003), weighted for area

b $P$ values from $\chi^{2}$ or ANOVA

The MAR was inversely associated with GDM risk ( $p$ for trend 0.011 ) adjusted for BMI, vitamin and mineral supplement use, smoking, physical activity, socio-demographic, reproductive and dietary factors (Table 6.4). Women in the quartile with the highest MAR had a 39\% lower risk of developing GDM compared to women in the lowest quartile ( $R R 0.61,95 \% \mathrm{Cl} 0.44-0.86$ ). Excluding the micronutrients from the MAR one by one did not change the results (data not shown). BMI, parity and educational level were no significant effect modifiers based on adding interaction terms to multivariable models ( $p$ value all $>0.20$ ). Similar associations were observed between inadequate micronutrient intakes, MAR and development of GDM in the sensitivity analyses performed (i.e. combining dietary intake dat a from surveys in 2003 and 2009, using multiple imputation for missing covariate data and excluding pregnancies occurring in the first 2 years of follow-up) (data not shown). 


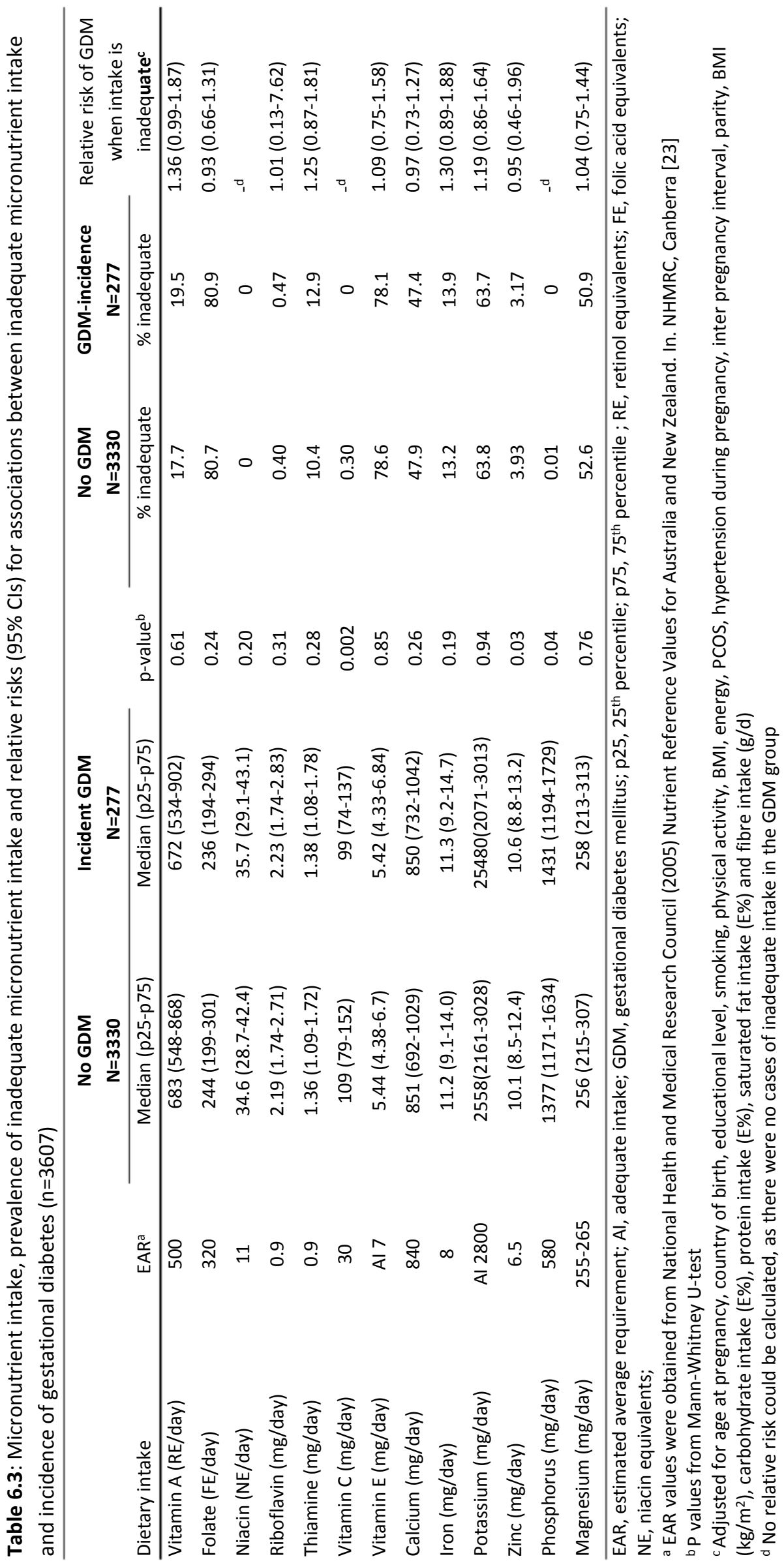


Table 6.4: Relative risks ( $95 \% \mathrm{Cls}$ ) for associations between mean micronutrient adequacy ratio and incidence of gestational diabetes $(n=3607)$.

\begin{tabular}{|c|c|c|c|c|c|}
\hline & \multicolumn{4}{|c|}{ Quartiles of mean adequacy ratio (MAR) } & \multirow{2}{*}{$\begin{array}{l}\mathrm{P} \text { for } \\
\text { trend }\end{array}$} \\
\hline & Quartile 1 & Quartile 2 & Quartile 3 & Quartile 4 & \\
\hline Median MAR & 0.81 & 0.87 & 0.90 & 0.95 & \\
\hline $\mathrm{N}$ women/pregnancies & $901 / 2084$ & $899 / 1290$ & $904 / 1242$ & $903 / 1647$ & \\
\hline GDM cases $n$ (\% pregnancies) & $112(5.4)$ & $66(5.1)$ & $55(4.4)$ & $52(3.2)$ & \\
\hline Model $1^{\mathrm{a}}$ & 1.00 (ref) & $1.05(0.79-1.43)$ & $0.91(0.66-1.25)$ & $0.57(0.40-0.80)$ & 0.001 \\
\hline Model $2^{\mathrm{b}}$ & 1.00 (ref) & $1.04(0.77-1.41)$ & $0.92(0.66-1.27)$ & $0.59(0.42-0.83)$ & 0.005 \\
\hline Model $3^{c}$ & 1.00 (ref) & $1.06(0.79-1.43)$ & $0.93(0.67-1.29)$ & $0.61(0.44-0.86)$ & 0.011 \\
\hline
\end{tabular}

a Adjusted for age, country of birth, educational level, vitamin and mineral supplement use, smoking, physical activity, energy, PCOS, hypertension during pregnancy, inter pregnancy interval and parity

${ }^{b}$ Model $1+$ additional adjustment for carbohydrate (E\%), protein (E\%), saturated fat $(E \%)$ and fibre $(\mathrm{g} / \mathrm{d})$

${ }^{\mathrm{c}}$ Model 2 + additional adjustment for BMI

\section{Discussion}

In our cohort of reproductive-aged women, prevalence of inadequate dietary micronutrient intake was more than $50 \%$ for the micronutrients calcium, potassium, magnesium, vitamin $\mathrm{E}$ and folate, indicating suboptimal pre-pregnancy micronutrient intakes. Inadequate micronutrient intake of individual nutrients was not associated with risk of developing GDM. However, women in the highest quartile of overall higher micronutrient intake as expressed by the MAR had a $39 \%$ lower risk of developing GDM compared to women in the lowest quartile and a declining trend over the quartiles was shown.

Maternal nutritional status during pregnancy is an essential factor in the health and development of their offspring, and thus having an adequate dietary intake of essential micronutrients is extremely important. However, as demonstrated by our study, women do not meet dietary reference values for a number of micronutrients in the years leading up to pregnancy, especially for folate. This was also observed in other studies $[37,38]$ including a recent study investigating micronutrient intake of Australian women before and during pregnancy [39]. The gap between recommended and actual dietary intake can be partly met by taking supplements. We had no information on frequency and dose of specific supplements and thus micronutrient intake in our study was based on dietary intake only. We did have information on vitamin and mineral supplement use, and observed associations between MAR and GDM were independent of reported vitamin and supplement use. It should be noted that women with a higher MAR were more likely to use vitamin or mineral supplements than women in the lowest quartile of MAR. This confirms results of previous research that those who need supplements the most (i.e. those with the lowest micronutrient intake) are the least likely to consume micronutrient supplements [39-41]. A recent study using data of 485 preconception women of the 
ALSWH study identified that $63 \%$ of the women used at least one supplement preconception and that $51 \%$ used a supplement containing folic acid [42]. This is in line with another Australian study that observed that $64 \%$ of the women took a dietary supplement in the preconception period, with $40 \%$ of the women using a supplement containing folic acid [39]. However, still a large proportion of women in this study did not achieve an adequate folate $(46 \%)$, iron $(80 \%)$ or zinc $(36 \%)$ intake in the preconception period. This underlines the need for further efforts to promote adequate dietary micronutrient intakes before pregnancy.

It should be noted that $40 \%$ of the pregnancies included in the current analyse were after 2009 . In 2009 folic acid fortification of flour was started. This was not taken into account in our dietary intake estimates of folate. Fortification increases dietary folate intakes with approximately $150 \mu \mathrm{g}$ for women of childbearing age [43] and is therefore expected to substantially decrease prevalence of inadequate folate intake to approximately $11 \%$ in this study population.

In our study, we observed no significant associations between intakes of individual micronutrients and risk of developing GDM. This was furthermore supported by the fact that excluding each micronutrient from the MAR one by one did not affect the results. This indicates that not one single micronutrient was driving the observed association between MAR and GDM. In contrast to the results of our study, other studies did report associations between intakes of individual micronutrients and risk of developing GDM. A recent review summarized the limited evidence suggesting an association between higher intake of iron, particularly heme iron, and higher risk of GDM [44]. In our study, we observed a $30 \%$ increased risk of GDM in women with inadequate iron intakes, but this was not statistically significant, and we were not able to distinguish between heme and non-heme iron intakes. It highlights, however, the need to further investigate iron intake in relation to GDM risk. Especially, since iron supplementation during pregnancy is recommended when iron deficiency anaemia is suspected $(9-37 \%$ of pregnant women $[38,45])$. Furthermore, one study observed a lower risk of GDM with higher intake of vitamin C [16]. This is in line with our observation that women who developed GDM had lower pre-pregnancy vitamin C intake compared to those who did not. However, intakes of vitamin $\mathrm{C}$ were adequate in both women who developed GDM and those with did not and we could not calculate a relative risk of GDM when vitamin C intake was inadequate. Another study observed a lower risk of GDM with higher plasma concentrations of zinc or selenium [12]. It should be noted that some of the strongest observed associations in these studies were associations using biomarkers indicating nutrient status instead of dietary intake. Unfortunately, we had no information on nutrient status, which might reflect nutrient stores better than dietary intakes and includes information on supplement intake and fortification. 
To study micronutrient adequacy, we used a summary measure of micronutrient intake across 13 micronutrients, i.e. the MAR, and observed an overall higher micronutrient intake to be associated with a lower risk of developing GDM. To our knowledge, no other studies investigated overall micronutrient adequacy and developing GDM. However, several studies investigated pre-pregnancy dietary patterns and risk of GDM $[33,35,46]$. Those studies, in general, observed a lower risk of GDM with dietary patterns reflecting high intakes of nutritious foods such as fruit, vegetables, whole grains and low-fat dairy (e.g. Mediterranean dietary pattern, prudent dietary pattern). Although adherence to a dietary pattern high in nutritious foods does not necessarily mean that recommended micronutrient intakes are met, it is associated with higher micronutrients intakes [26]. The observed relationship between dietary patterns high in nutritious foods and lower risk of GDM are thus in line with our observed relationship between micronutrient adequacy and lower risk of GDM.

Our study had several strengths. The longitudinal design of the study allowed us to examine associations between micronutrient adequacy and risk of GDM prospectively. In addition, information on 13 micronutrients and a wide variety of possible confounders was available. Finally, the design of the study enabled us to study pre-pregnancy dietary intake and included all pregnancies, including unplanned pregnancies.

However, some limitations need to be acknowledged. Data used in this study were self-reported. Selfreport could have led to misclassification of both the exposure and outcome. However, a reliability study among a subgroup of 1914 women from New South Wales demonstrated 91\% agreement between self-reported GDM diagnosis in our study and administrative data records [11]. In addition, the FFQ was validated against 7-day weighted food records in 63 Australian women. Energy-adjusted correlation coefficients for the micronutrients showed good to moderate agreement between the FFQ and the food records (correlation between 0.40-0.70), except for vitamin A (correlation coefficient 0.28) [20]. Furthermore, the MAR was not weighted, assuming equal importance of the different micronutrients. The MAR is a summary measure of overall micronutrient intake relative to recommended intakes, i.e. micronutrient adequacy, and therefore weighing was judged inappropriate. 


\section{Conclusions}

Pre-pregnancy dietary micronutrient intakes were suboptimal in this cohort of Australian women. A higher overall dietary micronutrient intake was associated with a lower risk of developing GDM, whereas inadequate intakes of individual micronutrient intakes were not associated with risk of GDM. This highlights the importance of an overall adequate micronutrient intake in the pre-pregnancy period. Future studies should investigate whether interventions improving overall dietary micronutrient adequacy before pregnancy reduce the risk of GDM and whether supplements could potentially play a role in improving overall micronutrient adequacy and, consequently, lower risk of GDM.

Acknowledgements: The research on which this paper is based was conducted as part of the Australian Longitudinal Study on Women's Health by the University of Queensland and the University of Newcastle. We are grateful to the Australian Government Department of Health for funding and to the women who provided the survey data.

The authors thank Professor Graham Giles of the Cancer Epidemiology and Intelligence Division of Cancer Council Victoria, for permission to use the Dietary Questionnaire for Epidemiological Studies (Version 2), Melbourne: Cancer Council Victoria, 1996. We also thank all the participants for their valuable contribution to this project.

Funding: The Australian Longitudinal Study on Women's Health was conceived and developed at the Universities of Newcastle and Queensland and is funded by the Australian Government Department of Health. G.D.M is supported by the Australian National Health and Medical Research Council Principal Research Fellowship (APP1121844). The Jo Kolk study fund is gratefully acknowledged for proving ML with a travel grant to visit the University of Queensland to conduct this research.

Confilicts of interest: The authors declare that there is no duality of interest associated with their contribution to this manuscript. 


\section{References}

1. Berti, C., H.K. Biesalski, R. Gartner, et al., Micronutrients in pregnancy: current knowledge and unresolved questions. Clin. Nutr., 2011. 30(6): p. 689-701.

2. McArdle, H.J. and C.J. Ashworth, Micronutrients in fetal growth and development. Br. Med. Bull., 1999. 55(3): p. 499-510.

3. MRC Vitamin Study Research Group, Prevention of neural tube defects: results of the Medical Research Council Vitamin Study. Lancet, 1991. 338(8760): p. 131-7.

4. Bardenheier, B.H., G. Imperatore, S.M. Gilboa, et al., Trends in Gestational Diabetes Among Hospital Deliveries in 19 U.S. States, 2000-2010. Am. J. Prev. Med., 2015. 49(1): p. 12-9.

5. Buckley, B.S., J. Harreiter, P. Damm, et al., Gestational diabetes mellitus in Europe: prevalence, current screening practice and barriers to screening. A review. Diabet. Med., 2012. 29(7): p. 844-54.

6. McCurdy CE and Friedman JE, Mechanisms underlying insulin resistance in human pregnancy and gestational diabetes mellitus., in Gestational diabetes during and after pregnancy, Kim C and Ferrara A, Editors. 2010, SpringerVerlag: London. p. p. 125-138.

7. Schoenaker, D.A., G.D. Mishra, L.K. Callaway, et al., The Role of Energy, Nutrients, Foods, and Dietary Patterns in the Development of Gestational Diabetes Mellitus: A Systematic Review of Observational Studies. Diabetes Care, 2016. 39(1): p. 16-23.

8. Zhang, C. and Y. Ning, Effect of dietary and lifestyle factors on the risk of gestational diabetes: review of epidemiologic evidence. Am. J. Clin. Nutr., 2011. 94(6 Suppl): p. 1975S-1979S.

9. Heer, M. and S. Egert, Nutrients other than carbohydrates: their effects on glucose homeostasis in humans. Diabetes Metab. Res. Rev., 2015. 31(1): p. 14-35.

10. Moore, W.T., S.M. Bowser, D.W. Fausnacht, et al., Beta Cell Function and the Nutritional State: Dietary Factors that Influence Insulin Secretion. Curr. Diab. Rep., 2015. 15(10): p. 76.

11. Russell, W.R., A. Baka, I. Bjorck, et al., Impact of Diet Composition on Blood Glucose Regulation. Crit. Rev. Food Sci. Nutr., 2016. 56(4): p. 541-90.

12. Bo, S., A. Lezo, G. Menato, et al., Gestational hyperglycemia, zinc, selenium, and antioxidant vitamins. Nutrition, 2005. 21(2): p. 186-91.

13. Bowers, K., E. Yeung, M.A. Williams, et al., A prospective study of prepregnancy dietary iron intake and risk for gestational diabetes mellitus. Diabetes Care, 2011. 34(7): p. 1557-63.

14. Qiu, C., C. Zhang, B. Gelaye, et al., Gestational diabetes mellitus in relation to maternal dietary heme iron and nonheme iron intake. Diabetes Care, 2011. 34(7): p. 1564-9.

15. Zhang, C., M.A. Williams, I.O. Frederick, et al., Vitamin C and the risk of gestational diabetes mellitus: a case-control study. J. Reprod. Med., 2004. 49(4): p. 257-66.

16. Zhang, C., M.A. Williams, T.K. Sorensen, et al., Maternal plasma ascorbic Acid (vitamin C) and risk of gestational diabetes mellitus. Epidemiology, 2004. 15(5): p. 597-604.

17. Brown, W.J., L. Bryson, J.E. Byles, et al., Women's Health Australia: recruitment for a national longitudinal cohort study. Women Health, 1998. 28(1): p. 23-40.

18. Lee, C., A.J. Dobson, W.J. Brown, et al., Cohort Profile: the Australian Longitudinal Study on Women's Health. Int. J. Epidemiol., 2005. 34(5): p. 987-91.

19. Huang, T.T., S.B. Roberts, N.C. Howarth, et al., Effect of screening out implausible energy intake reports on relationships between diet and BMI. Obes. Res., 2005. 13(7): p. 1205-17.

20. Hodge, A., A.J. Patterson, W.J. Brown, et al., The Anti Cancer Council of Victoria FFQ: relative validity of nutrient intakes compared with weighed food records in young to middle-aged women in a study of iron supplementation. Aust. N. Z. J. Public Health, 2000. 24(6): p. 576-83.

21. Ireland, P., D. Jolley, G. Giles, et al., Development of the Melbourne FFQ: a food frequency questionnaire for use in an Australian prospective study involving an ethnically diverse cohort. Asia Pac. J. Clin. Nutr., 1994. 3(1): p. 19-31.

22. Lewis, J., G. Milligan, and A. Hunt, Nuttab95: Nutrient data table for use in Australia. 1995, Australian Government Publishing Service: Canberra.

23. National Health and Medical Research Council, Nutrient Reference Values for Australia and New Zealand. 2005, NHMRC: Canberra.

24. Carriquiry, A.L., Assessing the prevalence of nutrient inadequacy. Public Health Nutr., 1999. 2(1): p. 23-33.

25. Krebs-Smith, S.M., H. Smiciklas-Wright, H.A. Guthrie, et al., The effects of variety in food choices on dietary quality. J. Am. Diet. Assoc., 1987. 87(7): p. 897-903.

26. Castro-Quezada, I., B. Roman-Vinas, and L. Serra-Majem, The Mediterranean diet and nutritional adequacy: a review. Nutrients, 2014. 6(1): p. 231-48.

27. Willett, W.C., G.R. Howe, and L.H. Kushi, Adjustment for total energy intake in epidemiologic studies. Am. J. Clin. Nutr., 1997. 65(4 Suppl): p. 1220S-1228S; discussion 1229S-1231S.

28. Hoffman, L., C. Nolan, J.D. Wilson, et al., Gestational diabetes mellitus--management guidelines. The Australasian Diabetes in Pregnancy Society. Med. J. Aust., 1998. 169(2): p. 93-7.

29. Gresham, E., P. Forder, C.L. Chojenta, et al., Agreement between self-reported perinatal outcomes and administrative data in New South Wales, Australia. BMC Pregnancy Childbirth, 2015. 15: p. 161. 
30. Brown, W.J., N.W. Burton, A.L. Marshall, et al., Reliability and validity of a modified self-administered version of the Active Australia physical activity survey in a sample of mid-age women. Aust. N. Z. J. Public Health, 2008. 32(6): p. 535-41.

31. Hanley, J.A., A. Negassa, M.D. Edwardes, et al., Statistical analysis of correlated data using generalized estimating equations: an orientation. Am. J. Epidemiol., 2003. 157(4): p. 364-75.

32. Spiegelman, D. and E. Hertzmark, Easy SAS calculations for risk or prevalence ratios and differences. Am. J. Epidemiol., 2005. 162(3): p. 199-200.

33. Schoenaker, D.A., S.S. Soedamah-Muthu, L.K. Callaway, et al., Pre-pregnancy dietary patterns and risk of gestational diabetes mellitus: results from an Australian population-based prospective cohort study. Diabetologia, 2015. 58(12): p. 2726-35.

34. Tryggvadottir, E.A., H. Medek, B.E. Birgisdottir, et al., Association between healthy maternal dietary pattern and risk for gestational diabetes mellitus. Eur. J. Clin. Nutr., 2016. 70(2): p. 237-42.

35. Tobias, D.K., C. Zhang, J. Chavarro, et al., Prepregnancy adherence to dietary patterns and lower risk of gestational diabetes mellitus. Am. J. Clin. Nutr., 2012. 96(2): p. 289-95.

36. Berglund, P., An introduction to multiple imputation of complex sample data using SAS v9.2, in SAS Global Forum Proceedings. 2010, SAS Institute Inc.: Cary, NC.

37. Blumfield, M.L., A.J. Hure, L. Macdonald-Wicks, et al., A systematic review and meta-analysis of micronutrient intakes during pregnancy in developed countries. Nutr. Rev., 2013. 71(2): p. 118-32.

38. Dubois, L., M. Diasparra, B. Bedard, et al., Adequacy of nutritional intake from food and supplements in a cohort of pregnant women in Quebec, Canada: the 3D Cohort Study (Design, Develop, Discover). Am. J. Clin. Nutr., 2017. 106(2): p. 541-548.

39. Livock, M., P.J. Anderson, S. Lewis, et al., Maternal micronutrient consumption periconceptionally and during pregnancy: a prospective cohort study. Public Health Nutr., 2017. 20(2): p. 294-304.

40. Aronsson, C.A., K. Vehik, J. Yang, et al., Use of dietary supplements in pregnant women in relation to sociodemographic factors - a report from The Environmental Determinants of Diabetes in the Young (TEDDY) study. Public Health Nutr., 2013. 16(8): p. 1390-402.

41. Pouchieu, C., R. Levy, C. Faure, et al., Socioeconomic, lifestyle and dietary factors associated with dietary supplement use during pregnancy. PLoS One, 2013. 8(8): p. e70733.

42. McKenna, E., A. Hure, A. Perkins, et al., Dietary Supplement Use during Preconception: The Australian Longitudinal Study on Women's Health. Nutrients, 2017. 9(10).

43. Dugbaza, J. and J. Cunningham, Estimates of total dietary folic Acid intake in the Australian population following mandatory folic Acid fortification of bread. J. Nutr. Metab., 2012. 2012: p. 492353.

44. Zhang, C. and S. Rawal, Dietary iron intake, iron status, and gestational diabetes. Am. J. Clin. Nutr., 2017.

45. Clark, S.F., Iron deficiency anemia. Nutr. Clin. Pract., 2008. 23(2): p. 128-41.

46. Zhang, C., M.B. Schulze, C.G. Solomon, et al., A prospective study of dietary patterns, meat intake and the risk of gestational diabetes mellitus. Diabetologia, 2006. 49(11): p. 2604-13. 


\section{Supplementary material}

Supplemental table 6.1: Micronutrient intake of 3,607 non-pregnant Australian women according to quartile of mean adequacy ratio (MAR)

\begin{tabular}{|c|c|c|c|c|c|}
\hline \multirow[b]{2}{*}{ Dietary intake } & \multirow[b]{2}{*}{$\mathrm{EAR}^{\mathrm{a}}$} & \multicolumn{4}{|c|}{ Quartiles of mean adequacy ratio (MAR) } \\
\hline & & $\begin{array}{c}\text { Quartile } 1 \\
\mathrm{~N}=901\end{array}$ & $\begin{array}{c}\text { Quartile } 2 \\
N=899\end{array}$ & $\begin{array}{c}\text { Quartile } 3 \\
N=904\end{array}$ & $\begin{array}{c}\text { Quartile } 4 \\
N=903\end{array}$ \\
\hline Vitamin A (RE/day) & 500 & $584(465-754)$ & $682(563-878)$ & $714(575-917)$ & $745(617-897)$ \\
\hline Folate (FE/day) & 320 & $182(154-221)$ & $223(195-268)$ & $254(224-300)$ & $300(263-347)$ \\
\hline Niacin (NE/day) & 11 & $29.4(24.4-38.0)$ & $34.3(28.6-42.8)$ & $36.3(30.4-42.9)$ & $37.7(32.3-43.7)$ \\
\hline Riboflavin (mg/day) & 0.9 & $1.63(1.37-2.14)$ & $2.09(1.74-2.56)$ & $2.32(1.97-2.76)$ & $2.54(2.20-2.99)$ \\
\hline Thiamine (mg/day) & 0.9 & $1.07(0.87-1.41)$ & $1.31(1.09-1.65)$ & $1.43(1.19-1.74)$ & $1.56(1.31-1.88)$ \\
\hline Vitamin C (mg/day) & 30 & 85 (59-119) & $103(76-144)$ & $119(87-158)$ & $127(95-170)$ \\
\hline Vitamin E (mg/day) & Al 7 & $4.53(3.64-5.83)$ & $5.30(4.33-6.60)$ & $5.74(4.7-6.76)$ & $5.99(5.10-7.24)$ \\
\hline Calcium (mg/day) & 840 & $696(590-838)$ & $822(700-1004)$ & 910 (768-1076) & 963 (830-1087) \\
\hline Iron (mg/day) & 8 & $8.9(7.5-11.2)$ & $10.6(9.0-12.9)$ & $11.6(9.9-13.9)$ & $13.3(11.3-15.7)$ \\
\hline Potassium (mg/day) & Al 2800 & 2013 (1763-2393) & 2413 (2146-2916) & $2682(2383-3067)$ & $2881(2606-3187)$ \\
\hline Zinc (mg/day) & 6.5 & $9.1(7.4-11.6)$ & $10.2(8.6-12.8)$ & $10.6(8.9-12.8)$ & $10.8(9.2-12.4)$ \\
\hline Phosphorus (mg/day) & 580 & 1156 (996-1423) & 1336 (1154-1609) & 1442 (1262-1689) & $1506(1356-1690)$ \\
\hline Magnesium (mg/day) & $255-265$ & $201(176-241)$ & 237 (2123-283) & 266 (237-309) & $300(267-337)$ \\
\hline
\end{tabular}

Values are median (p25-p75)

EAR, estimated average requirement; $A I$, adequate intake; $p 25,25^{\text {th }}$ percentile; $p 75,75^{\text {th }}$ percentile ; RE, retinol equivalents; FE, folic acid equivalents; NE, niacin equivalents;

a EAR values were obtained from National Health and Medical Research Council (2005) Nutrient Reference Values for Australia and New Zealand. In. NHMRC, Canberra 
Micronutrient adequacy and GDM risk 



\section{Chapter 7}

Longitudinal study on micronutrient intake and status, diet quality and glucose tolerance from preconception to the second trimester of pregnancy

Moniek Looman, Anouk Geelen, Rahul A.K. Samlal, Rik Heijligenberg, Lia D.E. Wijnberger, Jacqueline M.T. Klein Gunnewiek, Michiel G.J. Balvers, Edith J.M. Feskens, 


\section{Abstract}

Objective: The aim of this research is firstly to describe changes in folate, vitamin B6, vitamin B12, vitamin $\mathrm{D}$ and iron intake, their status markers and diet quality from preconception to the second trimester of pregnancy, and secondly to examine the association of these micronutrient intakes, their status markers and diet quality with glucose tolerance during pregnancy.

Methods: Data from 91 women aged 18-40 years with either a wish to get pregnant within one year or those less than 24 weeks pregnant were collected longitudinally. Women with an increased risk of GDM were oversampled, whereas multifetal pregnancies were excluded. Women were measured at preconception ( $n=67), 12$ weeks of pregnancy $(n=47)$ and 24 weeks of pregnancy $(n=55)$. At each time point women underwent a fasting venipuncture and a 75-grams oral glucose tolerance test. Dietary intake was assessed at each time point with a validated food frequency questionnaire and two nonconsecutive 24-hour recalls. Adjusted repeated measures mixed models were used to assess longitudinal associations of micronutrient intakes, status markers and diet quality with glucose tolerance during pregnancy.

Results: Micronutrient intakes changed significantly throughout pregnancy, due to changes in supplemental intakes, whereas dietary micronutrient intakes and diet quality remained stable. Nutrient status levels changed significantly from preconception to the second trimester of pregnancy. For folate, vitamin B6 and vitamin D this could be partly explained by changes in intake. In general, fasting and 2-hour glucose levels and HbA1c levels were not associated with diet quality, micronutrient intake or status levels, except for a weakly inverse association of folate intake with 2-hour glucose levels $(\beta=-0.001 \mathrm{mmol} / \mathrm{L}$ for each FE $\mu \mathrm{g}(95 \% \mathrm{Cl}-0.001 ; 0.000) \mathrm{p}=0.052)$, and a weakly positive association between ferritin and 2-hour glucose levels $(\beta=0.009 \mathrm{mmol} / \mathrm{L}$ for each $\mu \mathrm{g} / \mathrm{L}$ increase $(95 \% \mathrm{Cl}$ $-0.00 ; 0.018) ; p=0.058)$. Diet quality was in a sensitivity analysis, excluding data from participants with only one measurement, inversely associated with fasting glucose $(\beta=-0.007 \mathrm{mmol} / \mathrm{L}$ for each DHD15index point, $95 \% \mathrm{Cl}-0.010 ;-0.0001) ; \mathrm{p}=0.024)$.

Conclusions: Micronutrient intakes and their status markers changed significantly during pregnancy. More research is needed with respect to the role of micronutrients in relation to GDM development. 


\section{Introduction}

Micronutrient levels may affect pregnancy outcomes through alterations in maternal and foetal metabolism, as micronutrients are involved in enzyme activity, signal transduction and transcription pathways and oxidative stress [1,2]. Adequate dietary intake and nutrient status during pregnancy are conditions needed to ensure optimal foetal development and birth outcomes [3, 4]. Despite wide availability of healthy and nutrient-rich foods in high-income countries, nutrient deficiencies remain as high-fat, high-sugar diets with low nutrient density are increasingly consumed [5]. A recent review indicated that pregnant women are at high risk of inadequate intakes of iron, folate and vitamin D [6]. As vitamin B6 and B12 are, like folate, cofactors in the one-carbon metabolism responsible for DNA and RNA synthesis and methylation [7], these micronutrients are of interest as well.

Micronutrient intake might also affect maternal health. Gestational diabetes mellitus (GDM), defined as hyperglycaemia with onset or first detection during pregnancy, is one of the most common metabolic pregnancy complications, affecting approximately $7 \%$ of pregnancies [8, 9]. Risk of GDM is linked with diet and micronutrient intake $[10,11]$. Large prospective cohort studies observed associations of preconception dietary intake with diagnosis of GDM [12-14], whereas randomized controlled trials investigated the effect of dietary counselling during pregnancy on GDM prevention [15-17]. Thus far, evidence has linked a healthy preconception diet with a decreased risk of GDM [18]. Only few studies have investigated the role of folate, vitamin B6, vitamin B12, vitamin $D$, and iron in relation to risk of GDM. These studies linked higher vitamin B12 and vitamin D status to a lower risk of GDM [19-22], whereas higher folate and iron status were associated with an increased risk of GDM $[21,23]$. However, it should be noted that most of these studies had a cross-sectional or retrospective design and did not account for changes in dietary intake or nutrient status throughout pregnancy.

Furthermore, when investigating dietary micronutrient intake during pregnancy, supplement use is an important factor to take into account. The WHO recommends daily iron and folic acid supplement use for pregnant women [24] and studies report that up to $90 \%$ of pregnant women use at least one supplement during pregnancy $[25,26]$. Ideally, when investigating micronutrient intakes in relation to health outcomes, micronutrient status should also be included, as it provides additional information on nutrient stores. However, maternal haemodynamic, cardiovascular, renal and gastrointestinal adaptations occur during pregnancy, which influences blood micronutrient levels. Few studies have described micronutrient status levels throughout pregnancy [27-31], and, to our knowledge, none has studied the impact of dietary intake and supplement use during pregnancy.

We aim to complement previous described research by 1) describing changes in selected micronutrients intake, i.e. folate, vitamin B6, vitamin B12, vitamin D and iron, their status markers and 
overall diet quality from preconception to the second trimester of pregnancy, and 2) examining the association of micronutrients and overall diet quality with glucose tolerance during pregnancy in a sample of healthy women at increased risk of GDM with a singleton pregnancy.

\section{Methods}

\section{Study design and subjects}

This study was performed using data of the GLIMP2 study; a small prospective cohort study aiming to assess the role of diet, nutrient status and other risk factors in the development of gestational diabetes mellitus (GDM). Women with a wish to get pregnant within one year or those less than 24 weeks pregnant were recruited between June 2015 and May 2017 at the Department of Gynaecology and Department of Internal Medicine at three non-university hospitals in the eastern part of the Netherlands: Gelderse Vallei Hospital (Ede); Rijnstate (Arnhem); and Slingeland (Doetinchem). Women with a higher risk of developing GDM (i.e. previous pregnancy with GDM or macrosomic infant or overweight/obese) were oversampled. Main inclusion criteria were age between 18 and 40 years, willing to get pregnant within one year or less than 24 weeks pregnant at time of recruitment, and competent to make their own decisions. Women were excluded when they were not able to read and speak Dutch. The Medical Ethics Committee of Wageningen University \& Research approved the study. All women gave their written informed consent before the start of the study.

Measurements took place before pregnancy (T0), at 12 weeks of gestation (T1), and at 24 weeks of gestation (T2). All participants filled out a baseline questionnaire at the start of the study. At each time point, participants visited one of the research centres. Measurements included anthropometrics, a fasting venipuncture followed by a 75-grams oral glucose tolerance test (OGTT) including a venipuncture 2 hours after the glucose load, filling out a food frequency questionnaire (FFQ) and questionnaires on lifestyle, health and pregnancy-related factors. Additionally, women were asked to fill out a 24-hour recall (24hR) on two non-consecutive days within four weeks after each visit.

Dependent on whether participants were pregnant at the start of the study and duration of gestation women started at either T0, T1 or T2. In total, 115 women were included for participation in the study (Figure 7.1). For the current analysis, we used T0-T2 data collected until August 2017. Seven women dropped out before completing any of the measurements, and 17 women were excluded for the current analyses because of missing data (blood measurements or dietary intake data), leaving a total of 91 women for the current analyses. Eleven women dropped out after the T0 measurement. Main reasons for drop out were lack of time, and burden of the OGTT. In total, 57 women completed the 
measurement at T0, 43 women completed a measurement at T1 and 47 women completed a T2 measurement. Forty-four women completed at least two measurements and 19 women completed all three measurements. Women who did not get pregnant after T0 measurements $(n=19)$ did not differ significantly regarding age, BMI, ethnicity, education level and smoking status from those who did get pregnant after T0 measurement $(n=34)$ (data not shown). Furthermore, women who completed two or three measurements $(n=44)$ did not differ regarding age, ethnicity, education level and smoking status from women who completed only one measurement $(n=47)$ (data not shown).

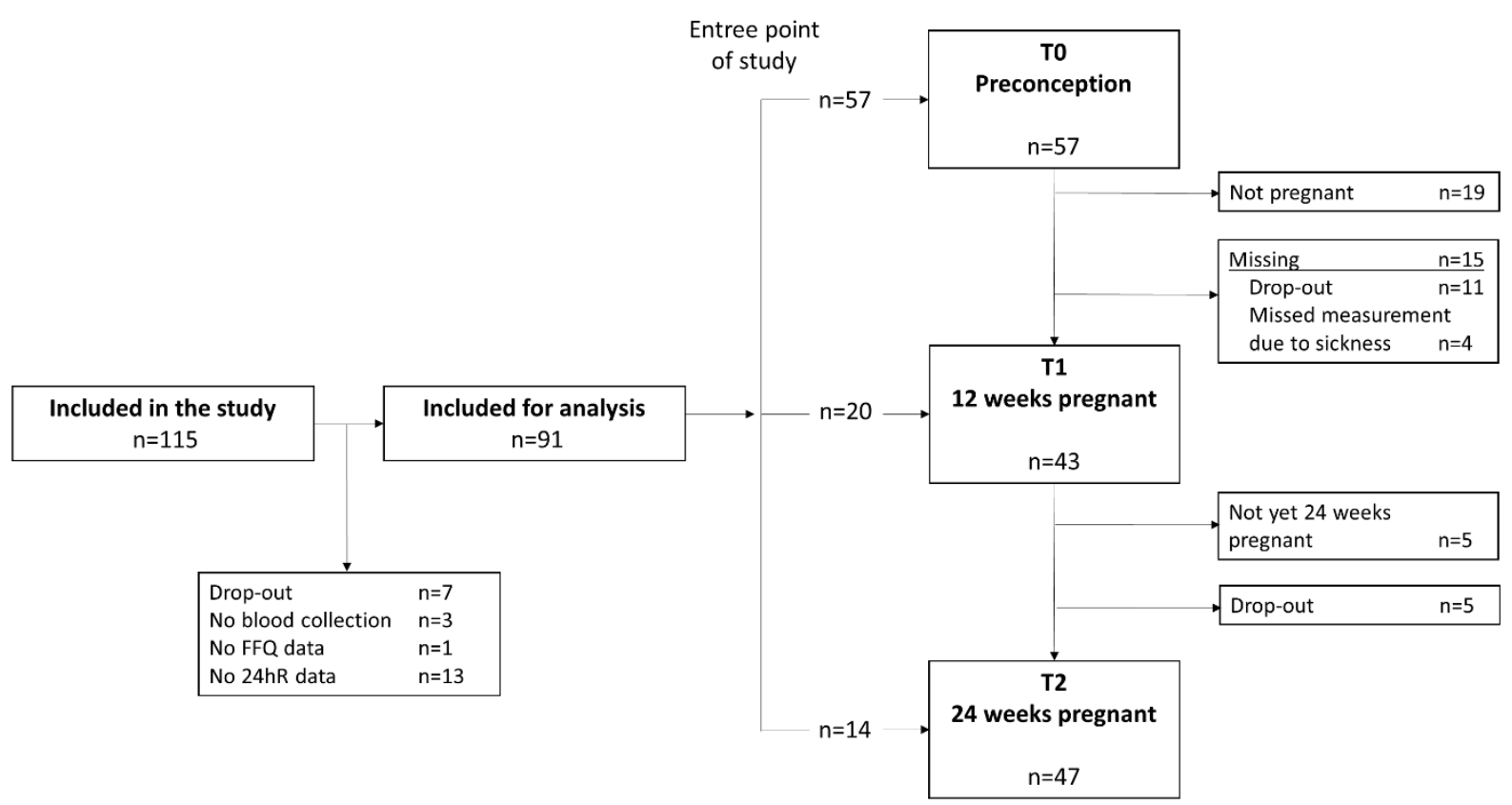

Figure 7.1: Flowchart of the GLIMP2 study.

\section{Dietary assessment}

\section{Food frequency questionnaire (FFQ)}

A semi-quantitative 173- item FFQ was used to assess usual dietary intake of the previous month. The FFQ was an updated version of a FFQ previously designed and validated to estimate habitual dietary intake of energy, macronutrients, fibre and B-vitamins in Dutch women of reproductive age [32-34]. Answer categories for frequency questions ranged between 'not in this month' to '6-7 days/week', and portion sizes were estimated using natural portions (bread shapes) and commonly used household measures (e.g. spoon and cup). Average daily nutrient intakes were calculated by multiplying frequency of consumption by portion size and nutrient content per gram using the 2011 Dutch food composition table [35]. 


\section{4 hour recalls (24hR)}

The $24 \mathrm{hR}$ were self-administered using the web-based program Compl-eat ${ }^{\mathrm{TM}}$ [36]. Unannounced invitations were sent via e-mail on two randomly selected days within four weeks after a completed measurement, with a median of 19 (interquartile range (IQR) 16) days between recalls. The web-based program Compl-eat ${ }^{\mathrm{TM}}$ guided participants to accurately report all foods and drinks consumed the previous day using the five-step multiple pass method [37]. Portion sizes were reported in commonly used household measures, standard portions, weight in grams, or volume in liters. Energy and nutrient intakes were estimated using the 2011 Dutch food composition table [35]. Trained dieticians checked all the $24 \mathrm{hRs}$ for their completeness and unusual portion sizes. Recalls were completed after the FFQ. Median time between completion of the FFQ and the first recall was 7 (IQR 6) days.

\section{Supplement use}

All participants were asked to report whether they used dietary supplements. For each supplement, the frequency, number of tablets or drops, type, and brand were reported. The nutrient content of the supplements was based on the product label information as obtained from the manufacturer. Total micronutrient intake for folate, vitamin B6, B12, D and iron was obtained by summing dietary intake and supplemental intake. To account for differences in bioavailability of natural and synthetic folate, folate intake was expressed as folate equivalents (FE). Total folate intake (FE $\mu \mathrm{g} /$ day) was obtained by summing dietary folate intake ( $\mathrm{FE} \mu \mathrm{g} / \mathrm{day})+1.7 *$ supplemental folic acid ( $\mu \mathrm{g} / \mathrm{day}$ ) [38].

\section{Dutch Healthy Diet 2015 (DHD15) index score}

The Dutch Healthy Diet index 2015 (DHD15-index) was used as measure of diet quality. Dietary intake data from the FFQ was used to calculate the scores. This index was developed based on the Dutch dietary guidelines of 2015 [39] and its design and calculation have been described elsewhere [40]. In brief, the DHD15-index comprises of 15 components on fruits, vegetables, wholegrain products, legumes, nuts, fish, tea, dairy, coffee, fats and oils, red meat, processed meat, sweetened beverages and fruit juices, alcohol and salt. The coffee and sodium component were omitted, as the type of coffee and sodium intake were not assessed. The scores for each component ranged between 0 and 10 points, resulting in a total DHD15-index score ranging from zero to 130 points. Higher scores indicate a higher level of adherence to the Dutch dietary guidelines.

\section{Combining FFQ and $24 \mathrm{hR}$ data}

To partly correct for measurement error present in dietary intake estimates, we used the enhanced regression calibration approach to combine dietary intake data obtained with FFQ and $24 \mathrm{hR}$. This approach has been described in detail elsewhere [41]. In brief, enhanced regression calibration is a simple extension of classic regression calibration using a random effect mixed model approach. The 
random effect estimate provides additional individual information as assessed with the $24 \mathrm{hR}$ measurement that is lost in classical regression calibration. The ERC approach was used for all dietary intake estimates including energy, macro- and micronutrient intake. However, the ERC method cannot be used to for the DHD15-index, as the DHD15 index is calculated based on individual intakes of 15 food groups, and the ERC method is not suited to correct individual intakes. As several components are episodically consumed foods, which cannot be accurately assessed with only two $24 \mathrm{hR}$, we based the DHD15-index score on FFQ data.

\section{Biochemical analysis}

Fasting blood samples were obtained by venipuncture in the morning at one of the hospitals followed by a 75-grams OGTT. Blood samples were transported in a cool storage box with a temperature around $7^{\circ} \mathrm{C}$ to the laboratory of Gelderse Vallei Hospital (Ede, the Netherlands) and processed within three hours after collection. Plasma fasting and 2-h glucose levels, fasting folate, ferritin and vitamin B12 were analysed using the Siemens Dimension Vista ${ }^{\circledR}$ System, a quantitative, competitive chemiluminescence immunoassay method based on $\mathrm{LOCI}^{\circledR}$ technology (Siemens Healthcare, The Hague, the Netherlands). Plasma ferritin was used as marker for iron status. Whole blood of the participant was used to measure HbA1c with the HA-8180V analyser (Menarini Diagnostics, Florence, Italy). Whole blood vitamin B6 concentration was measured with a validated Isocratic HPLC system with UV detector (Chromsystems Instruments \& Chemicals HmbH, Gräfelfing, Germany) till March 2017 and after March 2017 a liquid chromatography-mass spectrometry (LCMS) consisting of a Waters Acquity UPLC I-Class system, coupled to a Waters Xevo TQ-S micro mass spectrometer (Waters Corporation, Millford, Massachusetts, USA) was used. Serum 25(OH)D levels were measured using the HPLC system with UV detector as described above till July 2016 and after July 2016 a method using the above-described the LCMS system was used.

\section{Covariates}

Body weight and height were measured by trained professionals at each visit. Body Mass Index (BMI) was calculated as body weight divided by squared body height $\left(\mathrm{kg} / \mathrm{m}^{2}\right)$. Data on maternal age, ethnicity (western/non-western), marital status (married/living together), parity (no/one or more child), educational level (low/intermediate/high), and smoking habits (yes/no), nausea and vomiting during pregnancy were collected using standardized questionnaires. Birth country of the participant and her biologic parents was used to determine ethnicity. Highest completed education was classified into three categories: low: primary school, vocational or lower general secondary education, intermediate: higher secondary education or intermediate vocational training and high: higher vocational education or university. Physical activity was assessed with the validated Short QUestionnaire to Assess Healthenhancing physical activity (SQUASH) [42]. The durations (minutes per week) and intensity (Total 
Metabolic Equivalents (MET)) of total and light-, moderate-, and vigorous-intensity physical activities were calculated. Date of blood sampling was used to define a covariate for season (summer: May November and winter: December - April) [43]. A participant was diagnosed with GDM if at least one test value from the OGTT performed at 12 weeks of pregnancy or 24 weeks of pregnancy was abnormal (fasting glucose plasma $\geq 6.1 \mathrm{mmol} / \mathrm{L}$ or 2 -hour plasma glucose $\geq 7.8 \mathrm{mmol} / \mathrm{L}$ ), according to the diagnostic criteria of the WHO established in 1999 [44] .

\section{Statistical analysis}

Participant characteristics of the study population at each time point were reported as median (IQR), or as percentage (\%). Spearman's rank correlation coefficients were used to determine the correlation between measurements at different time points, as the distribution of most variables was skewed. To describe changes of micronutrient status, total intake, dietary intake and supplemental intake of folate, vitamin, B6, B12, D and iron during pregnancy a repeated measures mixed model with time as fixed effect was used, i.e. crude model. Repeated measures mixed models can account for missing observations and correlated measurements [45]. The covariance structure that resulted in the best model fit was chosen for each analysis. To assess whether dietary or supplemental intake was associated with (changes in) micronutrient status these variables were analysed in a model with micronutrient status as dependent outcome variable, and included covariates that influenced effect estimates, i.e. fully adjusted model. Potential covariates included age, education, ethnicity, parity, smoking, nausea during pregnancy, vomiting during pregnancy, season of blood collection, physical activity, energy intake, alcohol intake, time between measurements, BMI, and intakes and status of the other micronutrients.

To determine if micronutrient intakes, micronutrient status and diet quality were associated with markers of glucose tolerance, these were analysed as independent variables with glucose tolerance markers as dependent outcome variable. Covariates that significantly affected effect estimates were included in the model to adjust for confounding. Potential confounders included age, education, ethnicity, parity, smoking, season of blood collection, physical activity, energy intake, alcohol intake, time between measurements, history of GDM and BMI.

To assess if the large proportion of women who completed only one measurement ( $n=47$ out of $n=91$ ) affected results, we compared sociodemographic characteristics between women who completed only one measurement $(n=47)$ and women who completed at least two measurements $(n=41)$ and performed a sensitivity analysis using only data from participants who completed at least two measurements ( $n=44$ out of $n=91$ ). 
Statistical analyses were conducted using SAS Software Version 9.4 (SAS Institute Inc., Cary, NC, USA). A p-value of $\leq 0.05$ (two-sided) was considered statistically significant.

\section{Results}

Participants ( $n=91$ ) were on average $32.2 \pm 4.3$ years old, and mostly highly educated (67.0\%). Four of the 91 women (4.4\%) had a non-western ethnicity and the majority of the women (92.3\%) were multipara, i.e. had one or more children. Characteristics of the study population at each measurement moment are presented in Table 7.1. Over the course of pregnancy, median BMI increased from 24.3 (IQR 5.3) $\mathrm{kg} / \mathrm{m}^{2}$ at preconception until $26.8(7.6) \mathrm{kg} / \mathrm{m}^{2}$ at 24 weeks of pregnancy with high correlations between measurements $\left(r_{\text {TO-т1 }} 0.966\right.$ and $r_{\text {T1-т2 }}$ 0.985). Similar trends were observed for waist- and hipcircumference. Physical activity decreased after women got pregnant and women were on average the least physically active at 12 weeks of pregnancy. Measures of physical activity were moderately correlated ( $r_{T_{\mathrm{TO}-\mathrm{T} 1}} 0.424$ and $\mathrm{r}_{\mathrm{T} 1-\mathrm{T} 2}$ 0.379). Most women $(90 \%)$ experienced nausea and 30\% suffered from vomiting at 12 weeks gestation. These numbers decreased at the second trimester. Energy intake increased during pregnancy from 7691 (1469) kJ at preconception to 8764 (1609) kJ at 24 weeks of gestation. The relative contribution of proteins, carbohydrates and fat consumed remained quite stable throughout pregnancy. Median alcohol consumption before pregnancy was $0.6(4.0) \mathrm{g} / \mathrm{d}$ with $59.7 \%$ drinking no alcohol at all. During pregnancy, only two women reported consumption of a small amount of alcohol.

Changes in micronutrient intake, micronutrient status and diet quality throughout pregnancy Mean changes in micronutrient intake, micronutrient status and diet quality during pregnancy are presented in Figure 7.2, whereas the estimates of the adjusted repeated mixed model are presented in Table 7.2.

Total folate intake was highest at $\mathrm{T} 1$, due to a significant average increase in supplemental folate intake between T0 and T1 (Figure 7.2a). At T2, total and supplemental folate intakes were as low as preconception levels. Dietary folate intake remained stable from T0 to T2. Plasma folate levels increased significantly from $30.7 \pm 2.3 \mathrm{nmol} / \mathrm{L}$ at $\mathrm{TO}$ to $42.0 \pm 2.3$ at $\mathrm{T} 1$, and subsequently significantly decreased significantly to To levels (Supplemental table 7.1). Supplemental folate intake was significantly associated with plasma folate levels $(\beta 0.03 \mathrm{nmol} / \mathrm{L}$ for each $\mathrm{FE} \mu \mathrm{g}, 95 \% \mathrm{Cl}$ 0.025-0.041, $p<0.001$ ) (Table 7.2). Adjustment for supplemental folate intake attenuated the time effect in plasma folate, which became non-significant, suggesting that the observed change in plasma folate was due to changes in supplemental intake. 
Table 7.1: Characteristics of the study population according to measurement moment: preconception (T0), 12 weeks gestation (T1) and 24 weeks gestation (T2), including correlation between T0 and T1 measures and T1 and $\mathrm{T} 2$ measures.

\begin{tabular}{|c|c|c|c|c|c|c|}
\hline & $\begin{array}{l}\text { Total } \\
\mathrm{N}=91\end{array}$ & $\begin{array}{l}\text { T0 } \\
\mathrm{N}=57\end{array}$ & $\begin{array}{l}\mathrm{T} 1 \\
\mathrm{~N}=43\end{array}$ & $\begin{array}{l}\mathrm{T} 2 \\
\mathrm{~N}=47\end{array}$ & $\begin{array}{l}\mathrm{r}_{\mathrm{TO}-\mathrm{T} 1}{ }^{1} \\
\mathrm{n}=23\end{array}$ & $\begin{array}{l}r_{\mathrm{T} 1-\mathrm{T} 2}{ }^{1} \\
\mathrm{n}=31\end{array}$ \\
\hline Gestational age & - & $-13(24)$ & $12(3)$ & $24(2)$ & - & - \\
\hline Age (yrs) & $32.2(4.3)$ & $31.8(4.4)$ & $32.6(4.1)$ & $32.9(4.3)$ & 0.996 & 0.999 \\
\hline \multicolumn{7}{|l|}{ Educational level (\%) } \\
\hline - Low & 2.2 & 1.8 & 4.7 & 4.3 & \multirow{3}{*}{-} & \multirow{3}{*}{-} \\
\hline - $\quad$ Moderate & 30.8 & 26.3 & 25.6 & 29.8 & & \\
\hline - High & 67.0 & 71.9 & 69.8 & 65.9 & & \\
\hline Western ethnicity (\%) & 95.6 & 96.5 & 97.7 & 95.7 & - & - \\
\hline Smokers (\%) & 4.1 & 8.8 & 2.5 & 0 & - & - \\
\hline Parity $(\% \geq 1$ child & 92.3 & 91.2 & 95.4 & 91.5 & - & - \\
\hline Nausea during pregnancy (\%) & - & - & 90.0 & 53.2 & - & - \\
\hline $\begin{array}{l}\text { Vomitting during pregnancy } \\
(\%)\end{array}$ & - & - & 30.0 & 17.0 & - & - \\
\hline $\mathrm{BMI}\left(\mathrm{kg} / \mathrm{m}^{2}\right)$ & $24.9(6.4)$ & $24.3(5.3)$ & $24.7(9.8)$ & $26.8(7.6)$ & 0.966 & 0.985 \\
\hline Waist circumference $(\mathrm{cm})$ & $88.5(15.6)$ & $84.0(13.5)$ & $87.5(12.5)$ & $97.3(15.2)$ & 0.956 & 0.914 \\
\hline Hip circumference (cm) & $106.1(13.0)$ & $105.3(12.8)$ & $106.4(15.3)$ & $109.0(16.1)$ & 0.978 & 0.956 \\
\hline $\begin{array}{l}\text { Physical activity (MET } \\
\text { min/week) }\end{array}$ & 1215 (1279) & $1380(1183)$ & $930(518)$ & $980(998)$ & 0.424 & 0.379 \\
\hline Energy intake (kJ) & 7972 (1746) & 7691 (1469) & 8347 (2747) & 8764 (1609) & 0.735 & 0.330 \\
\hline Carbohydrates (E\%) & $48.4(4.9)$ & $48.2(5.2)$ & $49.0(3.9)$ & $48.7(4.4)$ & 0.510 & 0.774 \\
\hline Fat (E\%) & $33.5(3.8)$ & $33.5(4.2)$ & $33.4(2.9)$ & $33.7(3.6)$ & 0.453 & 0.742 \\
\hline Protein (E\%) & $15.3(1.6)$ & $15.5(1.9)$ & $15.2(1.8)$ & $14.7(1.9)$ & 0.620 & 0.567 \\
\hline Alcohol (g/d) & $0.1(1.3)$ & $0.6(4.0)$ & $0(0.5)$ & $0(0.5)$ & 0.413 & 0.542 \\
\hline $\begin{array}{l}\text { Blood sampling between } \\
\text { December and April (\%) }\end{array}$ & 24.5 & 15.8 & 30.2 & 29.8 & - & - \\
\hline
\end{tabular}

Values are median (IQR) or percentage

${ }^{1}$ Spearman rank correlations

Total, dietary and supplemental vitamin B6 intake did not significantly change from T0 to T2 (Figure 7.2b). Vitamin B6 levels decreased slightly from $89.9 \pm 3.9 \mathrm{nmol} / \mathrm{L}$ at $\mathrm{TO}$ to $88.4 \pm 3.3 \mathrm{nmol} / \mathrm{L}$ at $\mathrm{T} 1$, and decreased significantly to $78.3 \pm 3.4 \mathrm{nmol} / \mathrm{L}$ at $\mathrm{T} 2$. Both supplemental and dietary vitamin $\mathrm{B} 6$ intake were positively associated with vitamin B6 levels (Table 7.2). The observed significant decrease in vitamin B6 levels from T1 to T2 remained significant after adjustment for vitamin B6 intake.

Total vitamin B12 decreased slightly from T0 to T1 and subsequently decreased significantly from T1 to 12 , due to a significant decrease in supplemental vitamin B12 intake. Dietary vitamin B12 intake decreased slightly, but not significantly, from T0 to T2 (Figure 7.2c). Vitamin B12 levels significantly decreased from on average $308.2 \pm 11.7 \mathrm{pmol} / \mathrm{L}$ at T0 to $264.8 \pm 12.3 \mathrm{nmol} / \mathrm{L}$ at $\mathrm{T} 1$ and further decreased to $210.3 \pm 8.8 \mathrm{nmol} / \mathrm{L}$ at $\mathrm{T} 2$. This time effect was not explained by supplemental and dietary vitamin B12 intake as these were both not associated with vitamin B12 levels (Table 7.2). 

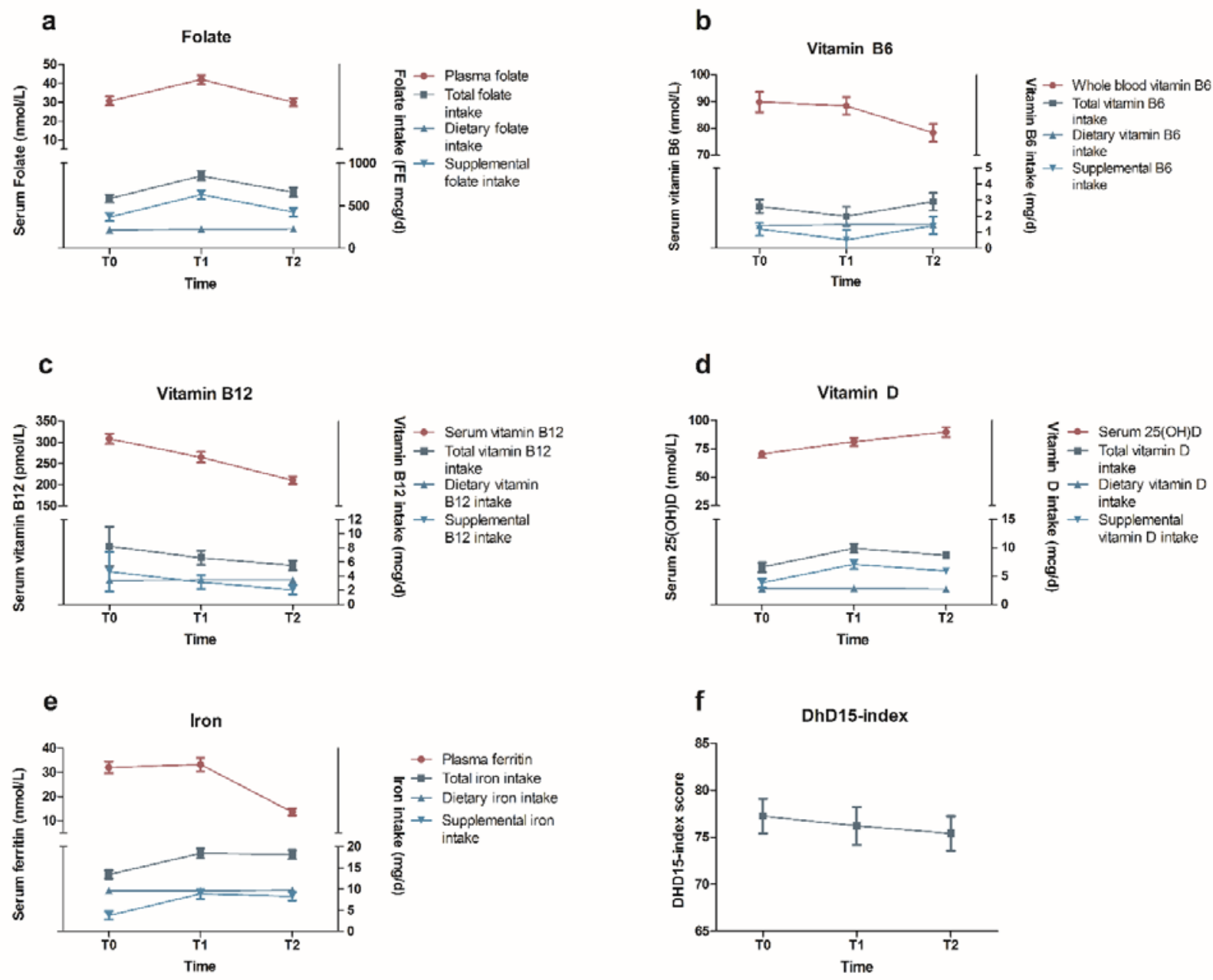

Figure 7.2: Changes in total, dietary and supplement nutrient intake and status for a) folate, b) vitamin B6, c) vitamin B12, d) vitamin D, e) iron, and f) diet quality as assessed by the DHD15-index. Values are mean (SEM). T0 is preconception, T1 is 12 weeks pregnant, and T2 is 24 weeks pregnant.

Total vitamin D intake significantly increased over the course of the pregnancy, due to a significant increase in supplemental vitamin D intake (Figure 7.2d). Observed supplemental vitamin D intake was on average highest at $\mathrm{T} 1$, whereas dietary vitamin $\mathrm{D}$ intake remained stable throughout pregnancy. Serum 25(OH)D levels, adjusted for season, significantly increased throughout pregnancy, from $70.4 \pm 3.2 \mathrm{nmol} / \mathrm{L}$ at $\mathrm{T} 0$ to $81.3 \pm 3.6$ at $\mathrm{T} 1$ and $89.9 .0 \pm 4.6 \mathrm{nmol} / \mathrm{L}$ at $\mathrm{T} 2$. Supplemental vitamin $\mathrm{D}$ intake was significantly associated with $25(\mathrm{OH}) \mathrm{D}$ serum levels $(\beta 1.17 \mathrm{nmol} / \mathrm{L}$ per $1 \mu \mathrm{g}$ supplemental vitamin $\mathrm{D}, 95 \% \mathrm{Cl}$ 0.50-1.81, $\mathrm{p}=0.001$ ) (Table 7.2). After adjustment for dietary and supplemental vitamin $\mathrm{D}$ intake, time was still significant, indicating that supplemental vitamin D intake could only partly explain the observed increase in $25(\mathrm{OH}) \mathrm{D}$ serum levels. 
Table 7.2: Regression coefficients $(\beta)$ of association of pregnancy (time), dietary intake and supplemental intake with changes in folate, vitamin B6, vitamin B12, 25(OH)D and ferritin blood levels.

\begin{tabular}{|c|c|c|c|c|}
\hline Outcome & Characteristic & $\beta$ & $95 \% \mathrm{Cl}$ & P-value \\
\hline \multirow[t]{4}{*}{ Folate status ${ }^{\mathrm{b}}(\mathrm{nmol} / \mathrm{l})$} & Time -12 weeks pregnancy ${ }^{a}$ & 3.25 & $-5.64 ; 64.6$ & 0.099 \\
\hline & Time -24 weeks pregnancy ${ }^{a}$ & -1.69 & $-1.84 ; 8.36$ & 0.206 \\
\hline & Supplemental folate intake (FE $\mu \mathrm{g}$ ) & 0.023 & $0.016 ; 0.029$ & $<0.001$ \\
\hline & Dietary folate intake (FE $\mu \mathrm{g}$ ) & -0.01 & $-0.06 ; 0.05$ & 0.836 \\
\hline \multirow[t]{4}{*}{ Vitamin B6 status ${ }^{\mathrm{C}}(\mathrm{nmol} / \mathrm{L})$} & Time -12 weeks pregnancy ${ }^{a}$ & -2.06 & $-10.8 ; 6.68$ & 0.638 \\
\hline & Time -24 weeks pregnancy ${ }^{a}$ & -10.9 & $-19.4 ;-2.45$ & 0.013 \\
\hline & Supplemental vitamin B6 intake (mg) & 2.23 & $0.88 ; 3.59$ & 0.002 \\
\hline & Dietary vitamin B6 intake (mg) & 14.9 & $0.58 ; 29.2$ & 0.042 \\
\hline \multirow[t]{4}{*}{ Vitamin B12 status ${ }^{\mathrm{d}}(\mathrm{pmol} / \mathrm{L})$} & Time -12 weeks pregnancy ${ }^{\mathrm{a}}$ & -55.3 & $-76.3 ;-34.3$ & $<0.001$ \\
\hline & Time -24 weeks pregnancy ${ }^{a}$ & -100.3 & $-121.3 ;-79.2$ & $<0.001$ \\
\hline & Supplemental vitamin B12 intake $(\mu \mathrm{g})$ & 0.12 & $-1 ; 01 ; 1.25$ & 0.832 \\
\hline & Dietary vitamin B12 intake ( $\mu \mathrm{g})$ & 6.66 & $-17.9 ; 31.3$ & 0.589 \\
\hline \multirow[t]{4}{*}{$25(\mathrm{OH}) \mathrm{D}$ status $^{\mathrm{e}}(\mathrm{nmol} / \mathrm{L})$} & Time -12 weeks pregnancy ${ }^{a}$ & 12.9 & $4.36 ; 21.5$ & 0.004 \\
\hline & Time -24 weeks pregnancy ${ }^{a}$ & 27.2 & $17.7 ; 36.7$ & $<0.001$ \\
\hline & Supplemental vitamin D intake $(\mu \mathrm{g})$ & 1.17 & $0.50 ; 1.84$ & 0.001 \\
\hline & Dietary vitamin $D$ intake $(\mu \mathrm{g})$ & 4.09 & $-1.86 ; 10.0$ & 0.174 \\
\hline \multirow[t]{4}{*}{ Ferritin status ${ }^{f}(\mu \mathrm{g} / \mathrm{L})$} & Time -12 weeks pregnancy ${ }^{a}$ & -4.33 & $-12.4 ; 3.69$ & 0.284 \\
\hline & Time -24 weeks pregnancy ${ }^{a}$ & -22.1 & $-28.6 ;-15.6$ & $<.001$ \\
\hline & Supplemental iron intake (mg) & 0.12 & $-0.18 ; 0.42$ & 0.416 \\
\hline & Dietary iron intake (mg) & -1.69 & $-4.30 ; 0.93$ & 0.201 \\
\hline
\end{tabular}

\footnotetext{
a Preconception is reference category.

${ }^{\mathrm{b}}$ Estimates are adjusted for age, education level, season of blood collection, BMI, energy intake, vitamin B12 and B6 intake.

c Estimates are adjusted for age, educational level, vitamin B12 intake and energy intake.

d Estimates are adjusted for parity, season of blood collection, BMI, energy intake, vitamin B6 intake.

e Estimates are adjusted for education level, parity, season of blood collection, BMI and energy intake.

${ }^{\mathrm{f}}$ Estimates are adjusted for alcohol intake, nausea during pregnancy, BMI and energy intake.
} 
Total iron intake increased from T0 to T2, due to a significant increase from T0 to T1. This was attributable to a significant increase in supplemental iron intake from T0 to $\mathrm{T} 1$, while dietary iron intake remained stable (Figure 7.2e). Ferritin levels remained stable from T0 to T1 $(32.0 \pm 2.4 \mathrm{nmol} / \mathrm{L}$ and $33.2 \pm 2.8 \mathrm{nmol} / \mathrm{L}$, resp.) but significantly decreased to $13.5 \pm 1.5 \mathrm{nmol} / \mathrm{L}$ at $\mathrm{T} 2$. Supplemental and dietary iron intake were not associated with ferritin levels and could not explain the time effect (Table 7.2).

Diet quality as reflected by the DHD15-index score decreased not significantly from $77.3 \pm 1.8$ points at preconception to $75.4 \pm 2.0$ at 24 weeks of pregnancy $(p=0.72)$ (Figure 7.2f).

Associations of micronutrient intake, micronutrient status and diet quality with glucose tolerance during pregnancy

In total, nine of the 91 (9.9\%) women developed GDM. Two women were diagnosed with GDM at 12 weeks of pregnancy and the other seven at 24 weeks of pregnancy. In general, micronutrient intake and status levels were not significantly associated with glucose tolerance over the course of the pregnancy (Table 7.3). Total folate intake was weakly inverse associated with 2-hour glucose levels ( $\beta=-0.001 \mathrm{mmol} / \mathrm{L}$ for each $\mathrm{FE} \mu \mathrm{g}, 95 \% \mathrm{Cl}-0.001-0.000 ; p=0.052$ ), whereas plasma ferritin levels was weakly positive associated with 2-hour glucose levels $(\beta=0.009 \mathrm{mmol} / \mathrm{L}$ for each $\mu \mathrm{g} / \mathrm{L}$ increase, $95 \% \mathrm{Cl}$ 0.00-0.018, $p=0.058$ ). A higher diet quality was associated with lower fasting glucose, 2-hour glucose and $\mathrm{HbA1c}$ levels, but these associations did not reach statistical significance $(p>0.20)$.

\section{Sensitivity analyses}

Age, ethnicity, education level and smoking status did not differ between women who completed only one measurement $(n=47)$ compared to women who completed two or three measurements $(n=44)$ (data not shown). Furthermore, similar trends were observed regarding changes in micronutrient intake and status level, intake-status relationships and associations of micronutrient intake and status with glucose tolerance when the study population was limited to participants who completed at least two measurements $(n=44)$, with one exception; in the sensitivity analyses we observed a significant inverse association between the DHD15-index and fasting glucose $(\beta=-0.007 \mathrm{mmol} / \mathrm{L}$ for each DHD15index point, $95 \% \mathrm{Cl}-0.010 ;-0.0001) ; \mathrm{p}=0.024)$. 
Table 7.3: Regression coefficients ( $\beta$ ) of associations between pregnancy (model 1 ), diet quality as assessed with DHD15-index (model 2), micronutrient intake (model 3) and micronutrient status (model 4) and fasting glucose, $2 \mathrm{~h}$ glucose levels and HbA1c.

\begin{tabular}{|c|c|c|c|c|c|}
\hline Outcome & Model & Exposure variable & $\beta$ & $95 \% \mathrm{Cl}$ & P-value \\
\hline Fasting glucose & $1)^{b}$ & Time -12 weeks pregnancy ${ }^{a}$ & -0.24 & $-0.37 ;-0.11$ & 0.001 \\
\hline \multirow[t]{12}{*}{$(\mathrm{mmol} / \mathrm{L})$} & & Time - 24 weeks pregnancy ${ }^{a}$ & -0.42 & $-0.57 ;-0.28$ & $<0.001$ \\
\hline & $2)^{c}$ & DHD-15 index score & -0.003 & $-0.01 ; 0.00$ & 0.219 \\
\hline & $3)^{c}$ & Total folate intake (FE $\mu \mathrm{g}$ ) & 0.0001 & $-0.001 ; 0.0003$ & 0.433 \\
\hline & & Total vitamin B6 intake (mg) & 0.016 & $-0.001 ; 0.040$ & 0.179 \\
\hline & & Total vitamin B12 intake $(\mu \mathrm{g})$ & -0.002 & $-0.008 ; 0.005$ & 0.614 \\
\hline & & Total vitamin D intake $(\mu \mathrm{g})$ & -0.0001 & $-0.013 ; 0.013$ & 0.990 \\
\hline & & Total iron intake (mg) & -0.003 & $-0.014 ; 0.008$ & 0.556 \\
\hline & $4)^{d}$ & Serum folate & -0.001 & $-0.007 ; 0.003$ & 0.845 \\
\hline & & Serum $25(\mathrm{OH}) \mathrm{D}$ & -0.000 & $-0.005 ; 0.005$ & 0.970 \\
\hline & & Whole blood vitamin B6 & 0.001 & $-0.000 ; 0.002$ & 0.172 \\
\hline & & Serum vitamin B12 & -0.000 & $-0.001 ; 0.001$ & 0.989 \\
\hline & & Serum ferritin & 0.000 & $-0.004 ; 0.004$ & 0.944 \\
\hline $2 \mathrm{~h}$ glucose & $1)^{b}$ & Time -12 weeks pregnancy ${ }^{a}$ & 0.16 & $-0.14 ; 0.45$ & 0.289 \\
\hline \multirow[t]{12}{*}{$(\mathrm{mmol} / \mathrm{L})$} & & Time -24 weeks pregnancy ${ }^{a}$ & 0.84 & $0.39 ; 1.27$ & $<0.001$ \\
\hline & $2)^{c}$ & DHD-15 index score & -0.005 & $-0.018 ; 0.007$ & 0.400 \\
\hline & $3)^{c}$ & Total folate intake (FE $\mu \mathrm{g}$ ) & -0.001 & $-0.001 ; 0.000$ & 0.052 \\
\hline & & Total vitamin B6 intake (mg) & -0.005 & $-0.060 ; 0.049$ & 0.851 \\
\hline & & Total vitamin B12 intake $(\mu \mathrm{g})$ & -0.001 & $-0.013 ; 0.011$ & 0.830 \\
\hline & & Total vitamin D intake ( $\mu \mathrm{g})$ & 0.005 & $-0.027 ; 0.036$ & 0.763 \\
\hline & & Total iron intake (mg) & 0.018 & $-0.009 ; 0.045$ & 0.192 \\
\hline & $4)^{d}$ & Serum folate & -0.004 & $-0.015 ; 0.008$ & 0.510 \\
\hline & & Serum $25(\mathrm{OH}) \mathrm{D}$ & -0.001 & $-0.008 ; 0.006$ & 0.883 \\
\hline & & Whole blood vitamin B6 & -0.001 & $-0.003 ; 0.002$ & 0.514 \\
\hline & & Serum vitamin B12 & -0.000 & $-0.002 ; 0.002$ & 0.776 \\
\hline & & Serum ferritin & 0.009 & $-0.00 ; 0.018$ & 0.058 \\
\hline $\mathrm{HbA1c}$ & $1)^{b}$ & Time -12 weeks pregnancy ${ }^{a}$ & -1.99 & $-2.72 ;-1.26$ & $<0.001$ \\
\hline \multirow[t]{12}{*}{$(\mathrm{mmol} / \mathrm{mol})$} & & Time -24 weeks pregnancy ${ }^{a}$ & -3.36 & $-4.08 ;-2.63$ & $<0.001$ \\
\hline & $2)^{c}$ & DHD-15 index score & -0.02 & $-0.05 ; 0.01$ & 0.255 \\
\hline & $3)^{c}$ & Total folate intake (FE $\mu \mathrm{g}$ ) & 0.001 & $-0.004 ; 0.002$ & 0.190 \\
\hline & & Total vitamin B6 intake (mg) & 0.060 & $-0.062 ; 0.182$ & 0.328 \\
\hline & & Total vitamin B12 intake $(\mu \mathrm{g})$ & 0.33 & $-0.034 ; 0.030$ & 0.802 \\
\hline & & Total vitamin D intake ( $\mu \mathrm{g})$ & 0.010 & $-0.064 ; 0.083$ & 0.795 \\
\hline & & Total iron intake (mg) & -0.015 & $-0.077 ; 0.048$ & 0.631 \\
\hline & $4)^{d}$ & Serum folate & 0.020 & $-0.007 ; 0.044$ & 0.158 \\
\hline & & Serum $25(\mathrm{OH}) \mathrm{D}$ & -0.002 & $-0.015 ; 0.012$ & 0.802 \\
\hline & & Whole blood vitamin B6 & -0.003 & $-0.009 ; 0.004$ & 0.419 \\
\hline & & Serum vitamin B12 & 0.003 & $-0.002 ; 0.008$ & 0.180 \\
\hline & & Serum ferritin & -0.013 & $-0.035 ; 0.009$ & 0.227 \\
\hline
\end{tabular}

\footnotetext{
a Preconception is reference category.

${ }^{\mathrm{b}}$ Adjusted for age, ethnicity, education level, parity, history of GDM, BMI

c Adjusted for time, age, ethnicity, parity, history of GDM, BMI, energy intake

d Adjusted for time, age, ethnicity, parity, history of GDM, BMI
} 


\section{Discussion}

In our longitudinal study, folate, vitamin B6, vitamin B12, vitamin D and iron intake changed significantly from preconception to 24 weeks of pregnancy. This was attributable to changes in supplemental intake. Folate, vitamin B6 and vitamin D intakes were significantly associated with their status markers. We observed a weakly inverse association between total folate intake and 2-hour glucose levels, whereas a weakly positive association was observed between serum ferritin with 2hour glucose levels. Other micronutrient intakes and status markers were not associated with glucose tolerance. Diet quality was in a sensitivity analysis, excluding data from participants with only one measurement, inversely associated with fasting glucose.

We observed that participants increased their energy intake on average with $14 \%$ from preconception to 24 weeks of pregnancy; however, we did not observe changes in macronutrient composition, dietary folate, vitamin B6, vitamin B12, vitamin D and iron intake, and diet quality. This is in line with other research investigating changes in dietary intake during pregnancy $[46,47]$. This is of concern, as pregnancy poses an important window of opportunity for improving diet quality [48-50], and recent studies have observed that women enter pregnancy with suboptimal micronutrient intakes $[6,25]$. Adequate micronutrient stores is one of the conditions needed to ensure an optimal intrauterine environment for foetal development [51, 52].

Supplementation can help in meeting recommendations and improving micronutrient status. Eightynine percent of the women in this study population used at least one supplement at 12 weeks of pregnancy. This is comparable with percentages reported in other studies $[25,26,53]$. The most commonly used supplements were folic acid, folic acid combined with vitamin D and prenatal multivitamin supplements. The usage, number of and type of supplements changed throughout pregnancy, with most pronounced the doubling of supplemental folate intake (mean $680 \mathrm{FE} \mu \mathrm{g}$ ) at 12 weeks of pregnancy compared to the mean $340 \mathrm{FE} \mu \mathrm{g}$ intake at preconception and 24 weeks of pregnancy. The observed drop in supplemental folate intake from 12 to 24 weeks of pregnancy could be explained by the recommendation to take folic acid supplements from at least 4 weeks before conception to 12 weeks of gestation [54], and is also observed in other studies [25, 26]. We observed substantial contributions from supplements to total micronutrient intakes. Considering that most women who take supplements are often women with a higher educational level, who, in general, already achieve higher intakes of micronutrients $[55,56]$, there is a risk of excess intake above the upper level of intake that might negatively affect health of the offspring. In our population, 13 women (14\%) consumed folate above the upper level of intake (1000 FE $\mu \mathrm{g}$ ). For other micronutrients, we observed no intake above upper levels. The intake of folate above the upper level of intake is of 
concern as a recent study has found a link between high doses of folic acid during pregnancy $(>5 \mathrm{mg} / \mathrm{d})$ and impaired psychomotor development at 12-23 months of age [57]. It should be noted that the maximum folate intake in our population was $1.6 \mathrm{mg} / \mathrm{d}$, but it indicates that excess intake should not be taken lightly and more research is needed to unravel the adverse effects of excess micronutrient intake, especially for potential adverse effects in the offspring.

We observed significant intake-status associations for folate, vitamin D and vitamin B6, but not for vitamin B12 and iron. This is in line with findings from validation studies in pregnant populations using nutritional biomarkers as reference instrument to validate dietary intake assessment methods [58]. The absence of an intake-status association for vitamin B12 might be due to a small range of intake, which limits the ability to detect an association. Ferritin levels is a marker of iron body stores in nonpregnant healthy individuals, but ferritin levels are during pregnancy affected by haemodilution, increased erythropoiesis and, pregnancy provoked acute-phase response [59] and might explain the absence of an association of ferritin with iron intakes. Supplemental folate intake was the strongest predictor for folate status levels. For the other micronutrients, intake could not (entirely) explain observed changes in status levels. In general, we observed that the status markers of water-soluble Bvitamins decreased from the end of the first trimester to the end of the second trimester. This decrease was likely attributable to haemodilution, the increase in blood volume in the second and third trimester $[28,60]$, in addition to changes in micronutrient intakes.

Vitamin $D$ is essential for bone development and has important immune functions [61]. Vitamin D deficiency is linked to adverse pregnancy-outcomes including preeclampsia, low birthweight, neonatal hypocalcaemia, poor postnatal growth and bone fragility [62]. In our study, vitamin D status, i.e. $25(\mathrm{OH}) \mathrm{D}$ levels, increased from preconception to 24 weeks of pregnancy. This is in line with findings of most $[31,63,64]$, but not all [65], other studies investigating vitamin D levels over the course of the pregnancy. This observed increase in $25(\mathrm{OH}) \mathrm{D}$ in our study remained after adjustments for dietary and supplemental intake, and other known potential confounders such as season, BMI and weight change. A possible explanation for the observed increase in $25(\mathrm{OH}) \mathrm{D}$ levels is increased production, potentially driven by placental vitamin D metabolism [66]. Worldwide, there has been increasing attention for the high prevalence of vitamin D deficiency in general and during pregnancy, with prevalences reported up to $84 \%$, depending on the country of residence [62]. However, cut-offs for vitamin D deficiency are derived on non-pregnant populations [67]. The observation of increasing 25(OH)D levels in our study and by others $[31,63,64]$ question the usability of these criteria in pregnant population as prevalence of deficiency might depend on the gestational age of the study population. 
Although the evidence regarding a beneficial effect of a healthy diet in GDM prevention is growing, research regarding micronutrient intake and status is limited. We observed no significant associations, micronutrient intake or status levels with glucose tolerance markers, except for a weakly inverse association between total folate intake and 2-hour glucose levels, a weakly positive association between serum ferritin with 2-hour glucose levels, and, in a sensitivity analysis, an inverse association between diet quality and fasting glucose. In contrast to our results, two cross-sectional Asian studies reported a higher risk of GDM with higher folate levels during pregnancy, and an inverse association for vitamin B12 status during pregnancy with GDM risk [20, 21]. A retrospective study in the UK also observed a lower vitamin B12 status in women with GDM, but did not find an association with of folate status with GDM risk [22]. Furthermore, several observational studies found an inverse association of vitamin D status during pregnancy with GDM development, but this could not be confirmed in randomized controlled trials [19] and in our longitudinal study, we also did not observe a significant association between vitamin $D$ intake, $25(\mathrm{OH}) \mathrm{D}$ status levels and markers of glucose tolerance.

A recent review concluded that there is a potential link between greater iron status and increased risk of GDM. Adequate iron status is critical to normal beta cell function and glucose homeostasis, but excess iron may disrupt glucose homeostasis, by damaging beta cell function, increasing oxidative stress and impaired insulin signalling [68-70]. Evidence is most consistent for iron stores, with higher iron stores being associated with higher risk of GDM [23]. This is in line with our weakly positive association of ferritin levels with 2-hour glucose levels. As iron supplementation is often prescribed to prevent or treat iron deficiency anaemia, the potential association of a higher GDM risk with higher iron status warrants more research.

Major strengths of the present study include its prospective design, inclusion of measurements before conception and detailed information on dietary intake, supplemental intake and micronutrient status markers. However, the present study also has limitations. Firstly, due to the extensive nature of our study, with measurements at preconception as well as during pregnancy, including a visit to the research centre, an OGTT and various dietary assessment at each time point, our sample size was limited, making our study potentially underpowered to assess significant associations. However, we were able to use continuous effect measures, and had extensive information regarding dietary intake, supplement intake, serum levels and potential confounders. Secondly, due to various reasons (women who did not get pregnant, sickness, holidays etc.), more than half of the participants completed only one measurement. However, we used a repeated measures mixed model to account for the missing data. In addition, similar trends and effect estimates were observed in our sensitivity analysis using data from women who completed two or three measurements. Thirdly, participants included in the present study were mainly highly educated, recruited in a certain region of the Netherlands and 
women with a high risk of GDM were oversampled. These factors may limit external validity to the general population of pregnant women. Lastly, as the primary outcome of the study was development of GDM, the last measurement took place at 24 weeks of pregnancy. To accurately describe changes in micronutrient levels over the pregnancy additional measurements after 24 weeks of pregnancy would give a more complete picture.

\section{Conclusions}

Micronutrient intake changes throughout pregnancy, due to changes in supplemental intake, whereas dietary micronutrient intake and diet quality remained on average stable. Nutrient status levels changed significantly from preconception to the second trimester of pregnancy, which could be partly explained by changes in intake from diet and supplements. This should be confirmed in future research, as well as the implication for micronutrient deficiency thresholds during pregnancy. We did not observe significant associations of micronutrient intake, status levels and diet quality with fasting, 2-hour glucose and HbA1c levels; except for except for a weakly inverse association between total folate intake and 2-hour glucose levels, a weakly positive association between serum ferritin with 2hour glucose levels, and, in a sensitivity analysis, an inverse association between diet quality and fasting glucose. More research is needed with respect to the role of micronutrients in relation to GDM development.

Acknowledgements: We thank all participants for their time and contributions, and the research staff and students for their help in this study. Furthermore, we would like to thank the departments of Gynaecology and Obstetrics and department of Internal Medicine of hospitals Gelderse Vallei, Ede; Rijnstate, Arnhem; and Slingeland, Doetinchem for their help in the recruitment of participants.

Funding: This work was supported by the Division of Human Nutrition, Wageningen University and the Clinical Chemistry and Haematology Laboratory, Hospital Gelderse Vallei Ede (biochemical analyses).

Conflict of interests: The authors declare that they have no conflicts of interest. 


\section{References}

1. McArdle, H.J. and C.J. Ashworth, Micronutrients in fetal growth and development. Br. Med. Bull., 1999. 55(3): p. 499-510.

2. Simpson, J.L., L.B. Bailey, K. Pietrzik, et al., Micronutrients and women of reproductive potential: required dietary intake and consequences of dietary deficiency or excess. Part II--vitamin D, vitamin A, iron, zinc, iodine, essential fatty acids. J. Matern. Fetal Neonatal Med., 2011. 24(1): p. 1-24.

3. Abu-Saad, K. and D. Fraser, Maternal nutrition and birth outcomes. Epidemiol. Rev., 2010. 32: p. 5-25.

4. Berti, C., H.K. Biesalski, R. Gartner, et al., Micronutrients in pregnancy: current knowledge and unresolved questions. Clin. Nutr., 2011. 30(6): p. 689-701.

5. Gernand, A.D., K.J. Schulze, C.P. Stewart, et al., Micronutrient deficiencies in pregnancy worldwide: health effects and prevention. Nat. Rev. Endocrinol., 2016. 12(5): p. 274-89.

6. Blumfield, M.L., A.J. Hure, L. Macdonald-Wicks, et al., A systematic review and meta-analysis of micronutrient intakes during pregnancy in developed countries. Nutr. Rev., 2013. 71(2): p. 118-32.

7. Locasale, J.W., Serine, glycine and one-carbon units: cancer metabolism in full circle. Nat. Rev. Cancer, 2013. 13(8): p. 572-83.

8. American Diabetes Association, Diagnosis and classification of diabetes mellitus. Diabetes Care, 2014. 37 Suppl 1: p. S81-90.

9. Buckley, B.S., J. Harreiter, P. Damm, et al., Gestational diabetes mellitus in Europe: prevalence, current screening practice and barriers to screening. A review. Diabet. Med., 2012. 29(7): p. 844-54.

10. Schoenaker, D.A., G.D. Mishra, L.K. Callaway, et al., The Role of Energy, Nutrients, Foods, and Dietary Patterns in the Development of Gestational Diabetes Mellitus: A Systematic Review of Observational Studies. Diabetes Care, 2016. 39(1): p. 16-23.

11. Zhang, C. and Y. Ning, Effect of dietary and lifestyle factors on the risk of gestational diabetes: review of epidemiologic evidence. Am. J. Clin. Nutr., 2011. 94(6 Suppl): p. 1975S-1979S.

12. Schoenaker, D.A., S.S. Soedamah-Muthu, L.K. Callaway, et al., Pre-pregnancy dietary patterns and risk of gestational diabetes mellitus: results from an Australian population-based prospective cohort study. Diabetologia, 2015. 58(12): p. 2726-35.

13. Tobias, D.K., C. Zhang, J. Chavarro, et al., Prepregnancy adherence to dietary patterns and lower risk of gestational diabetes mellitus. Am. J. Clin. Nutr., 2012. 96(2): p. 289-95.

14. Zhang, C., M.B. Schulze, C.G. Solomon, et al., A prospective study of dietary patterns, meat intake and the risk of gestational diabetes mellitus. Diabetologia, 2006. 49(11): p. 2604-13.

15. Koivusalo, S.B., K. Rono, M.M. Klemetti, et al., Gestational Diabetes Mellitus Can Be Prevented by Lifestyle Intervention: The Finnish Gestational Diabetes Prevention Study (RADIEL): A Randomized Controlled Trial. Diabetes Care, 2016. 39(1): p. 24-30.

16. Oostdam, N., M.N. van Poppel, M.G. Wouters, et al., Interventions for preventing gestational diabetes mellitus: a systematic review and meta-analysis. J Womens Health (Larchmt), 2011. 20(10): p. 1551-63.

17. Poston, L., R. Bell, H. Croker, et al., Effect of a behavioural intervention in obese pregnant women (the UPBEAT study): a multicentre, randomised controlled trial. The lancet. Diabetes \& endocrinology, 2015. 3(10): p. 767-77.

18. Donazar-Ezcurra, M., C. Lopez-del Burgo, and M. Bes-Rastrollo, Primary prevention of gestational diabetes mellitus through nutritional factors: a systematic review. BMC Pregnancy Childbirth, 2017. 17.

19. Burris, H.H. and C.A. Camargo, Jr., Vitamin D and gestational diabetes mellitus. Curr. Diab. Rep., 2014. 14(1): p. 451.

20. Krishnaveni, G.V., J.C. Hill, S.R. Veena, et al., Low plasma vitamin B12 in pregnancy is associated with gestational 'diabesity' and later diabetes. Diabetologia, 2009. 52(11): p. 2350-8.

21. Lai, J.S., W.W. Pang, S. Cai, et al., High folate and low vitamin B12 status during pregnancy is associated with gestational diabetes mellitus. Clin. Nutr., 2017.

22. Sukumar, N., H. Venkataraman, S. Wilson, et al., Vitamin B12 Status among Pregnant Women in the UK and Its Association with Obesity and Gestational Diabetes. Nutrients, 2016. 8(12).

23. Zhang, C. and S. Rawal, Dietary iron intake, iron status, and gestational diabetes. Am. J. Clin. Nutr., 2017.

24. World Health Organization, WHO recommendations on antenatal care for a positive pregnancy experience. 2016, World Health Organization: Geneva, Switzerland.

25. Dubois, L., M. Diasparra, B. Bedard, et al., Adequacy of nutritional intake from food and supplements in a cohort of pregnant women in Quebec, Canada: the 3D Cohort Study (Design, Develop, Discover). Am. J. Clin. Nutr., 2017. 106(2): p. 541-548. 
26. Livock, M., P.J. Anderson, S. Lewis, et al., Maternal micronutrient consumption periconceptionally and during pregnancy: a prospective cohort study. Public Health Nutr., 2017: p. 1-11.

27. Cikot, R.J., R.P. Steegers-Theunissen, C.M. Thomas, et al., Longitudinal vitamin and homocysteine levels in normal pregnancy. Br. J. Nutr., 2001. 85(1): p. 49-58.

28. Hure, A.J., C.E. Collins, and R. Smith, A longitudinal study of maternal folate and vitamin B12 status in pregnancy and postpartum, with the same infant markers at 6 months of age. Matern Child Health J, 2012. 16(4): p. 792-801.

29. Milman, N., A.O. Agger, and O.J. Nielsen, Iron supplementation during pregnancy. Effect on iron status markers, serum erythropoietin and human placental lactogen. A placebo controlled study in 207 Danish women. Dan. Med. Bull., 1991. 38(6): p. 471-6.

30. Milman, N., T. Bergholt, K.E. Byg, et al., Reference intervals for haematological variables during normal pregnancy and postpartum in 434 healthy Danish women. Eur. J. Haematol., 2007. 79(1): p. 39-46.

31. Zhao, Y., W. Miao, C. Li, et al., Dynamic changes in serum 25-hydroxyvitamin D during pregnancy and lack of effect on thyroid parameters. PLoS One, 2014. 9(3): p. e90161.

32. Siebelink, E., A. Geelen, and J.H. de Vries, Self-reported energy intake by FFQ compared with actual energy intake to maintain body weight in 516 adults. Br. J. Nutr., 2011. 106(02): p. 274-281.

33. Streppel, M.T., J.H. de Vries, S. Meijboom, et al., Relative validity of the food frequency questionnaire used to assess dietary intake in the Leiden Longevity Study. Nutr. J., 2013. 12: p. 75.

34. Verkleij-Hagoort, A.C., J.H. de Vries, M.P. Stegers, et al., Validation of the assessment of folate and vitamin B12 intake in women of reproductive age: the method of triads. Eur. J. Clin. Nutr., 2007. 61(5): p. 610-615.

35. NEVO-tabel, Dutch Food Composition Table 2011/version 3. 2011, National Institute for Publich Health and the Environment/Netherlands Nutrition Centre.

36. Meijboom, S., M. Van Houts-Streppel, C. Perenboom, et al., Evaluation of dietary intake assessed by the Dutch self-administered web-based dietary 24- $h$ recall tool (Compl-eat ${ }^{T M}$ ) against intervieweradministered telephone-based 24-h recalls. Journal of nutritional science, 2017. 6.

37. Conway, J.M., L.A. Ingwersen, B.T. Vinyard, et al., Effectiveness of the US Department of Agriculture 5step multiple-pass method in assessing food intake in obese and nonobese women. Am. J. Clin. Nutr., 2003. 77(5): p. 1171-8.

38. Institute of Medicine, Dietary Reference Intakes for Thiamin, Riboflavin, Niacin, Vitamin B6, Folate, Vitamin B12, Pantothenic Acid, Biotin, and Choline. 1998: Washington, D.C.

39. Health Council of the Netherlands, Dutch dietary guidelines 2015. Publication no. 2015/24. 2015, Health Council of the Netherlands: The Hague.

40. Looman, M., E.J. Feskens, M. de Rijk, et al., Development and evaluation of the Dutch Healthy Diet index 2015. Public Health Nutr., 2017. 20(13): p. 2289-2299.

41. Looman, M., H. Boshuizen, E. Feskens, et al., Using enhanced regression calibration to combine dietary intake estimates from 24-hour recall and food frequency questionnaire improves diet-disease associations. Submitted for publication, 2017.

42. Wendel-Vos, G.C., A.J. Schuit, W.H. Saris, et al., Reproducibility and relative validity of the short questionnaire to assess health-enhancing physical activity. J. Clin. Epidemiol., 2003. 56(12): p. 1163-9.

43. Brouwer-Brolsma, E.M., A.M. Vaes, N.L. van der Zwaluw, et al., Relative importance of summer sun exposure, vitamin D intake, and genes to vitamin D status in Dutch older adults: The B-PROOF study. The Journal of steroid biochemistry and molecular biology, 2016. 164: p. 168-176.

44. World Health Organization, Definition, diagnosis and classification of diabetes mellitus and its complications. 1999, World Health Organization: Geneva, Switzerland.

45. Cnaan, A., N.M. Laird, and P. Slasor, Using the general linear mixed model to analyse unbalanced repeated measures and longitudinal data. Stat. Med., 1997. 16(20): p. 2349-80.

46. Crozier, S.R., S.M. Robinson, K.M. Godfrey, et al., Women's dietary patterns change little from before to during pregnancy. J. Nutr., 2009. 139(10): p. 1956-63.

47. Gresham, E., C.E. Collins, G.D. Mishra, et al., Diet quality before or during pregnancy and the relationship with pregnancy and birth outcomes: the Australian Longitudinal Study on Women's Health. Public Health Nutr., 2016. 19(16): p. 2975-2983.

48. Blondin, J.H. and J.A. LoGiudice, Pregnant women's knowledge and awareness of nutrition. Appl. Nurs. Res., 2018. 39: p. 167-174.

49. Stephenson, J., D. Patel, G. Barrett, et al., How do women prepare for pregnancy? Preconception experiences of women attending antenatal services and views of health professionals. PLoS One, 2014. 9(7): p. e103085. 
50. Szwajcer, E., G.J. Hiddink, L. Maas, et al., Nutrition awareness before and throughout different trimesters in pregnancy: a quantitative study among Dutch women. Fam. Pract., 2012. 29 Suppl 1: p. i82-i88.

51. Kaiser, L., L.H. Allen, and American Dietetic Association, Position of the American Dietetic Association: nutrition and lifestyle for a healthy pregnancy outcome. J. Am. Diet. Assoc., 2008. 108(3): p. 553-61.

52. Williamson, C.S., Nutrition in pregnancy. Nutr. Bull., 2006. 31: p. 28-59.

53. McKenna, E., A. Hure, A. Perkins, et al., Dietary Supplement Use during Preconception: The Australian Longitudinal Study on Women's Health. Nutrients, 2017. 9(10).

54. MRC Vitamin Study Research Group, Prevention of neural tube defects: results of the Medical Research Council Vitamin Study. Lancet, 1991. 338(8760): p. 131-7.

55. Aronsson, C.A., K. Vehik, J. Yang, et al., Use of dietary supplements in pregnant women in relation to sociodemographic factors - a report from The Environmental Determinants of Diabetes in the Young (TEDDY) study. Public Health Nutr., 2013. 16(8): p. 1390-402.

56. Pouchieu, C., R. Levy, C. Faure, et al., Socioeconomic, lifestyle and dietary factors associated with dietary supplement use during pregnancy. PLoS One, 2013. 8(8): p. e70733.

57. Valera-Gran, D., M. Garcia de la Hera, E.M. Navarrete-Munoz, et al., Folic acid supplements during pregnancy and child psychomotor development after the first year of life. JAMA pediatrics, 2014. 168(11): p. e142611.

58. Ortiz-Andrellucchi, A., J. Doreste-Alonso, P. Henriquez-Sanchez, et al., Dietary assessment methods for micronutrient intake in pregnant women: a systematic review. Br. J. Nutr., 2009. 102 Suppl 1: p. S64-86.

59. Coad, J. and C. Conlon, Iron deficiency in women: assessment, causes and consequences. Curr. Opin. Clin. Nutr. Metab. Care, 2011. 14(6): p. 625-34.

60. Costantine, M.M., Physiologic and pharmacokinetic changes in pregnancy. Front. Pharmacol., 2014. 5 : p. 65.

61. Pludowski, P., M.F. Holick, S. Pilz, et al., Vitamin D effects on musculoskeletal health, immunity, autoimmunity, cardiovascular disease, cancer, fertility, pregnancy, dementia and mortality-a review of recent evidence. Autoimmunity reviews, 2013. 12(10): p. 976-89.

62. Dawodu, A. and C.L. Wagner, Mother-child vitamin D deficiency: an international perspective. Arch. Dis. Child., 2007. 92(9): p. 737-40.

63. Barebring, L., I. Schoenmakers, A. Glantz, et al., Vitamin D Status during Pregnancy in a Multi-Ethnic Population-Representative Swedish Cohort. Nutrients, 2016. 8(10).

64. Davies-Tuck, M., C. Yim, M. Knight, et al., Vitamin D testing in pregnancy: Does one size fit all? Aust. N. Z. J. Obstet. Gynaecol., 2015. 55(2): p. 149-55.

65. Zhang, J.Y., A.J. Lucey, R. Horgan, et al., Impact of pregnancy on vitamin D status: a longitudinal study. Br. J. Nutr., 2014. 112(7): p. 1081-7.

66. Park, H., M.R. Wood, O.V. Malysheva, et al., Placental vitamin D metabolism and its associations with circulating vitamin D metabolites in pregnant women. Am. J. Clin. Nutr., 2017. 106(6): p. 1439-1448.

67. Holick, M.F., Vitamin D deficiency. N. Engl. J. Med., 2007. 357(3): p. 266-81.

68. Cooksey, R.C., H.A. Jouihan, R.S. Ajioka, et al., Oxidative stress, beta-cell apoptosis, and decreased insulin secretory capacity in mouse models of hemochromatosis. Endocrinology, 2004. 145(11): p. 5305-12.

69. Fernandez-Real, J.M., D. McClain, and M. Manco, Mechanisms Linking Glucose Homeostasis and Iron Metabolism Toward the Onset and Progression of Type 2 Diabetes. Diabetes Care, 2015. 38(11): p. 216976.

70. Rajpathak, S.N., J.P. Crandall, J. Wylie-Rosett, et al., The role of iron in type 2 diabetes in humans. Biochim. Biophys. Acta, 2009. 1790(7): p. 671-81. 


\section{Supplementary material}

Supplemental table 7.1: Micronutrient intake, micronutrient status, diet quality and glucose tolerance markers of the study population according to measurement moment: preconception (T0), 12 weeks gestation (T1) and 24 weeks gestation (T2).

\begin{tabular}{|c|c|c|c|}
\hline & $\begin{array}{l}\text { T0 } \\
N=67\end{array}$ & $\begin{array}{l}\mathrm{T} 1 \\
\mathrm{~N}=47\end{array}$ & $\begin{array}{l}\mathrm{T} 2 \\
\mathrm{~N}=55\end{array}$ \\
\hline DHD15 score & $77.2(1.8)$ & $76.2(2.0)$ & $75.4(1.8)$ \\
\hline Plasma folate $(\mathrm{nmol} / \mathrm{L})$ & $30.7(2.3)$ & $42.0(2.3)$ & $30.0(2.1)$ \\
\hline Total folate intake (FE $\mu \mathrm{g}$ ) & $587(46)$ & $856(53)$ & $660(54)$ \\
\hline - Dietary intake & $216(5)$ & $226(7)$ & $229(7)$ \\
\hline - $\quad$ Supplemental intake & $368(45)$ & $631(52)$ & $429(52)$ \\
\hline Whole blood vitamin B6 (nmol/L) & 90 (3.9) & $88(3.3)$ & $78(3.4)$ \\
\hline Total vitamin B6 intake (mg) & $2.6(0.4)$ & $2.0(0.6)$ & $2.9(0.6)$ \\
\hline - $\quad$ Dietary intake & $1.4(0.04)$ & $1.5(0.04)$ & $1.5(0.04)$ \\
\hline - Supplemental intake & $1.2(0.4)$ & $0.5(0.6)$ & $1.4(0.6)$ \\
\hline Serum vitamin B12 (pmol/L) & $308(12)$ & $265(12)$ & $210(9)$ \\
\hline Total vitamin B12 intake ( $\mu \mathrm{g})$ & $8.2(2.8)$ & $6.6(1.0)$ & $5.5(0.7)$ \\
\hline Dietary intake & $3.4(0.1)$ & $3.5(0.1)$ & $3.5(0.1)$ \\
\hline - $\quad$ Supplemental intake & $4.7(2.8)$ & $3.1(1.0)$ & $2.1(0.7)$ \\
\hline Serum 25(OH)D (nmol/L) & $70(3.2)$ & $81(3.6)$ & $90(4.5)$ \\
\hline Total vitamin D intake ( $\mu \mathrm{g})$ & $6.6(0.8)$ & $9.9(0.8)$ & $8.7(0.6)$ \\
\hline Dietary intake & $2.8(0.1)$ & $2.9(0.1)$ & $2.8(0.1)$ \\
\hline - Supplemental intake & $3.8(0.8)$ & $7.1(0.8)$ & $5.9(0.6)$ \\
\hline Plasma ferritin $(\mu \mathrm{g} / \mathrm{L})$ & $32.0(2.4)$ & $33.1(2.8)$ & $13.5(1.5)$ \\
\hline Total iron intake (mg) & $13.4(1.0)$ & $18.4(1.2)$ & $18.1(1.1)$ \\
\hline Dietary intake & $9.6(0.1)$ & $9.6(0.2)$ & $9.8(0.2)$ \\
\hline - $\quad$ Supplemental intake & $3.8(1.0)$ & $8.6(1.2)$ & $8.3(1.0)$ \\
\hline Fasting glucose (mmol/L) & $4.8(0.1)$ & $4.6(0.1)$ & $4.5(0.1)$ \\
\hline Glucose $2 \mathrm{~h}$ after OGTT (mmol/L) & $4.7(0.1)$ & $4.9(0.2)$ & $5.9(0.1)$ \\
\hline $\mathrm{HBA} 1 \mathrm{c}(\mathrm{mmol} / \mathrm{mol})$ & $33.6(0.3)$ & $31.8(0.3)$ & $30.9(0.4)$ \\
\hline
\end{tabular}

Values are mean (SEM) 
Micronutrient intake, status, diet quality and glucose tolerance 



\section{Chapter 8}

Supplement use and dietary sources of folate, vitamin D, and $n-3$ fatty acids during preconception: the GLIMP2 study

Moniek Looman, Claudia van den Berg, Anouk Geelen, Rahul A.K. Samlal, Rik Heijligenberg, Jacqueline M.T. Klein Gunnewiek, Michiel G.J. Balvers, Caroline L. Leendertz-Eggen, Lia D.E. Wijnberger, Edith J.M. Feskens, Elske M. Brouwer-Brolsma 


\section{Abstract}

Background: An adequate nutritional status during the preconception period is important, particularly for folate, vitamin $D$, and $n-3$ fatty acids (i.e. EPA+DHA). We aimed to determine supplement intake and the main dietary sources of folate, vitamin D, and EPA+DHA. Additionally, associations of these intakes with their blood levels were examined.

Methods: Data of 66 Dutch women aged 18-40y who wished to become pregnant was used. Dietary intake was assessed with a validated food frequency questionnaire, supplement use with a structured questionnaire. 25-hydroxyvitamin $\mathrm{D}$ levels were determined in serum and folate and phospholipid EPA+DHA levels in plasma. Partial Spearman's correlations, restricted cubic splines and trend analyses over tertiles of nutrient intakes were performed to examine intake-status associations.

Results: A large proportion of women did not meet the Dutch recommended intakes of folate $(50 \%)$, vitamin D (67\%), and EPA+DHA (52\%). Vegetables were the main contributor to dietary folate intake (25\%), oils and fats to dietary vitamin D intake (39\%), and fish to dietary EPA+DHA intake (69\%). Fourteen percent of the women had an inadequate folate status and $23 \%$ an inadequate vitamin $D$ status. Supplemental folate intake, supplemental and dietary vitamin D intake and dietary EPA+DHA intake were significantly associated with their blood levels.

Conclusions: Even in our highly educated population a large proportion did not achieve recommended folate, vitamin D and $n-3$ fatty acid intakes. Promotion of folate and vitamin D supplement use and fish consumption is needed to improve intakes and blood levels of these nutrients in women who wish to become pregnant. 


\section{Introduction}

A woman's dietary intake and nutritional status before conception and during pregnancy are important determinants of maternal and foetal health, and child's health later in life [1-3]. Consequently, maternal dietary habits during the preconception period are considered increasingly important to cover the nutritional needs of the foetus and placenta during pregnancy [4], and to optimize maternal and infant health [5]. To promote the health of prospective parents, the Health Council of the Netherlands has published preconception care guidelines [6]. Despite these guidelines, recent studies showed that preconception dietary intake is still suboptimal with high prevalence of inadequate habitual diet and nutrient status $[5,7]$.

Preconception care dietary guidelines including recommendations on optimal intakes of folate, vitamin D, and fish (i.e. n-3 fatty acids eicosapentaenoic acid (EPA) and docosahexaenoic acid (DHA)). Recommendations regarding folate intake are predominantly based on the knowledge that an adequate folate intake in the preconception period reduces the risk of a neural tube defect [8]. Although folate naturally occurs in many dietary sources, such as green leafy vegetables, fruits, meat, and dairy products, it is difficult for Dutch women to obtain the recommended amounts of folate through diet alone [6]. Therefore, a daily folic acid supplement ( $\geq 400 \mu \mathrm{g}$ folic acid) is recommended as part of routine preconception and antenatal care [6].

An adequate preconception vitamin D status is important as vitamin D deficiency in the mother has been linked to various issues in the pregnancy such as preeclampsia [9] and gestational diabetes [10] and in their offspring such as low birth weight [11], poor bone growth [12], and an increased risk of recurrent wheeze or asthma $[13,14]$.. Sunlight is the predominant source of vitamin $D[15]$. Whereas dietary vitamin $\mathrm{D}$ can only be obtained through the consumption of a limited number of foods, such as fatty fish, fat spreads, oils, liver, meat, eggs, and dairy products. Therefore, vitamin D supplement use (i.e. $10 \mu \mathrm{g} / \mathrm{d}$ ) is recommended for Dutch women who wish to become pregnant and pregnant women, particularly during winter [16].

The $\mathrm{n}-3$ fatty acids EPA and DHA are considered to be important nutrients for the foetal brain and retina development [17]. Therefore, women are recommended to consume 200mg/day EPA+DHA [18], which translates into at least 1 portion of fatty fish/week [19].

Despite these recommendations, little is known about current intake levels of folate, vitamin $D$, and EPA+DHA among Dutch women who wish to become pregnant. Moreover, there is limited data on the relative contribution of these nutrients from dietary sources in relation to their blood levels in preconception women. More knowledge on these aspects may benefit further specification of the dietary recommendations in this field of practice. To address this perceived knowledge gap, we aimed 
to: (i) describe current intake levels of folate, vitamin $D$, and the $n-3$ fatty acids EPA+DHA from dietary sources and supplements; (ii) determine the relative contribution of the top- 5 of dietary sources to the total intake of these nutrients; (iii) examine how dietary and supplementary intakes of these nutrients relate to their blood levels; and (iv) identify which sources contribute the most to the nutritional status per nutrient.

\section{Methods}

\section{Study population}

This cross-sectional study was performed using baseline data of the GLIMP2 study; an observational prospective cohort study designed to assess the role of diet, nutrient status, and other risk factors in the development of gestational diabetes mellitus (GDM). Participants were recruited at the Department of Gynaecology and Obstetrics and Department of Internal Medicine at three nonuniversity hospitals in the eastern part of the Netherlands: Gelderse Vallei (Ede); Rijnstate (Arnhem); and Slingeland (Doetinchem). Main inclusion criteria were age between 18 and 40 years, willing to get pregnant within one year at the time of recruitment, and competent to make their own decisions. Women with a higher risk of developing GDM (i.e. previous pregnancy with GDM or macrosomic infant or overweight/obese) were oversampled in this study population. Women were excluded when they were not able to read and speak Dutch. Participants who were pregnant at time of recruitment were excluded for current analysis as information on preconception dietary intake was missing. Consequently, preconception dietary intake data and blood levels of folate, vitamin D and EPA+DHA were available for 66 women. The GLIMP2 study was conducted by Wageningen University \& Research between 2015 and 2017. The Medical Ethics Committee of Wageningen University \& Research approved the GLIMP2 study. All women gave their written informed consent before the start of the study.

\section{Dietary assessment}

Dietary intake was assessed with the use of a validated, semi-quantitative 173- item FFQ assessing habitual food and beverage intake of the previous month. The FFQ was an updated version of a FFQ previously designed and validated to estimate dietary intake of energy, macronutrients, and B-vitamins in Dutch women of reproductive age [20-22]. The FFQ included consumption frequencies (from once a month to several times a day) and the number of units eaten or portion sizes (e.g., slices, cups, pieces, spoons, etc.) according to Dutch household measures [23]. Food groups were created and the contribution of each food group to total dietary folate, vitamin D and EPA+DHA intake was calculated. All participants were asked to report whether they used dietary supplements. For each supplement, 
the frequency, number of tablets or drops, type, and brand were reported. The nutrient content of the supplements was based on the product label information as obtained from the manufacturer or ATC code website. Total nutrient intake was obtained by summing dietary intake and supplemental intake. To account for differences in bioavailability of natural and synthetic folate, folate intake was expressed as folate equivalents ( $\mathrm{FE}$ ). Total folate intake ( $\mathrm{FE} \mu \mathrm{g} / \mathrm{day}$ ) was obtained by summing dietary folate intake (FE $\mu \mathrm{g} /$ day) $+1.7 *$ supplemental folic acid $(\mu \mathrm{g} /$ day) [24]

\section{Biochemical analyses}

Fasting blood samples were obtained by venipuncture in the morning at one of the hospitals for assessing blood levels of folate, vitamin D, and EPA+DHA. Blood samples were transported in a cool storage box with a temperature around $7^{\circ} \mathrm{C}$ to the laboratory of Gelderse Vallei Hospital (Ede, the Netherlands) and processed within three hours after collection. Tubes with blood plasma containing EDTA for phospholipid fatty acid composition analyses were transported to the division of Human Nutrition of Wageningen University \& Research (Wageningen, the Netherlands) and stored at $-80^{\circ} \mathrm{C}$. Plasma folate was analysed using the Siemens Dimension Vista ${ }^{\circledR}$ folate method with the Dimension Vista ${ }^{\circledR}$ System, a quantitative, competitive chemiluminescence immunoassay method based on $\mathrm{LOCl}^{\circledR}$ technology (Siemens Healthcare, The Hague, the Netherlands). Plasma folate levels below $10 \mathrm{nmol} / \mathrm{L}$ were considered as folate insufficient [25]. Serum 25-hydroxyvitamin D (25(OH)D) was analysed using a validated isocratic High Performance Liquid Chromatography (HPLC) method using UV detection (Chromsystems Instruments \& Chemicals HmbH, Gräfelfing, Germany). Serum 25(OH)D levels below $50 \mathrm{nmol} / \mathrm{L}$ were considered as insufficient [26]. To assess EPA+DHA status, the fatty acid composition of EDTA plasma phospholipids were analysed in the laboratory of the division of Human Nutrition of Wageningen University \& Research. After isolation of the phospholipid fraction and saponification, the fatty acids were derivatized to fatty acid methyl esters (FAMEs), which were subsequently quantified with a gas chromatograph (Hewlett Packard 5890 Series II) equipped with a capillary column (WCOT fused silica kolom CP WAX 58, 25m x 0.25 mm id Chrompack CP 7717) and flame-ionization detection, using nitrogen as carrier gas. Fatty acid concentrations were reported as g/100g FAME; the sum of all peak areas of the fatty acids identified was set to 100\% [27]. For EPA+DHA there was no generally acceptable cut-off for an optimal EPA+DHA status.

\section{Covariates}

Anthropometric measurements were performed by trained professionals. Body weight was determined to the nearest $0.1 \mathrm{~kg}$ with a calibrated balance (SECA, Germany), while women had to take off their shoes and empty their pockets. Body height was measured with a wall-mounted stadiometer (SECA, Germany) to the nearest $0.1 \mathrm{~cm}$, while wearing no shoes. Body Mass Index (BMI) was calculated 
as body weight divided by squared body height $\left(\mathrm{kg} / \mathrm{m}^{2}\right.$ ). Data on maternal age, ethnicity (Western/nonWestern), marital status (married/living together), parity (no/one or more child), educational level (low/intermediate/high), smoking habits (yes/no), and alcohol consumption (g/day) were collected using standardized questionnaires. Data on birth country of the participant and birth country of her biologic parents was used to determine ethnicity (Western or non-Western). Educational level was evaluated based on the highest completed education and classified into three categories: low: primary school, vocational or lower general secondary education, intermediate: higher secondary education or intermediate vocational training and high: higher vocational education or university. Intakes of vitamin B6 and vitamin B12 were obtained from the FFQ and the questionnaire on supplement use. Date of blood sampling was used to define a covariate for season (summer: May - November and winter: December - April) [15].

\section{Statistical analysis}

Participant characteristics of the study population were reported as mean $\pm S D$, as median (interquartile rage (IQR)), or as $n(\%)$ for the total population. Additionally, we made two strata: one for women who did meet recommended intake and one for women who did not meet recommended intake $[6,16,18]$. We did this for folate ( $<680 \mathrm{FE} \mu \mathrm{g} / \mathrm{d}$ and $\geq 680 \mathrm{FE} \mu \mathrm{g} / \mathrm{d})$, vitamin $\mathrm{D}(<10 \mu \mathrm{g} / \mathrm{d}$ and $\geq 10$ $\mu \mathrm{g} / \mathrm{d}$ ), and EPA+DHA recommendations ( $<200 \mathrm{mg} / \mathrm{d}$ and $\geq 200 \mathrm{mg} / \mathrm{d}$ ). Independent sample $t$-tests, ChiSquare tests, and Mann Whitney $U$ tests were used to compare characteristics between participants who met and participants who did not meet the recommendation. Spearman's rank correlation coefficients and partial correlation coefficients were used to determine the correlation of total nutrient intake, total dietary intake, supplemental intake, and nutrient intake from the top-5 dietary sources with blood levels. Analysis of covariance (ANCOVA) was performed to compare blood levels across tertiles of nutrient intake and to calculate adjusted means with $95 \%$ confidence intervals. Additionally $P$ for trend analysis was performed using the median intake of the tertile as a continuous variable in the ANCOVA model. Restricted cubic splines were used to visualize the dose-response relationship between total nutrient intake and blood levels of folate, vitamin $D$, and EPA+DHA. Three knots were used at the $1^{\text {st }}, 5^{\text {th }}$, and $9^{\text {th }}$ decile of intake. Significant predictors for blood levels in linear regression models $(p<0.05)$ were included as covariates in the statistical analyses. For folate these were: season of blood sampling, total energy intake, total vitamin B6 intake and total vitamin B12 intake; for vitamin D and EPA+DHA these were: educational level, season of blood sampling, total energy intake and BMI. Statistical analyses were conducted using SAS Software Version 9.4 (SAS Institute Inc., Cary, NC, USA), except for the restricted cubic splines, which were performed using R statistical software version 3.1.1 and R Studio 1.0. A $p$-value of $\leq 0.05$ (two-sided) was considered statistically significant. 


\section{Results}

\section{Participant characteristics}

Characteristics of the total study population $(n=66)$ stratified by intake categories of folate, vitamin D, and EPA+DHA are shown in Table 8.1. The mean age of the total study population was $31.7 \pm 4.1$ years and mean BMI was $25.2 \pm 4.0 \mathrm{~kg} / \mathrm{m}^{2}$. Median (IQR) total folate intake was 713 (672) $\mathrm{FE} \mu \mathrm{g} / \mathrm{d}$, median total vitamin $D$ was 5.9 (8.5) $\mu \mathrm{g} / \mathrm{d}$, and median total EPA+DHA intake was 170 (200) mg/d. Thirty-seven women (56\%) used a folic acid supplement, thirty women (46\%) a vitamin D supplement, and three women (5\%) a supplement containing EPA+DHA.

Folate intake \& status

Half of the participants met the recommended daily folate intake ( $\geq 680 \mathrm{FE} \mu \mathrm{g} ; \mathrm{n}=33$ ). Women with an adequate folate intake had a significantly higher median (IQR) total folate intake (943 (124) FE $\mu \mathrm{g} / \mathrm{d}$ versus 272 (102) FE $\mu \mathrm{g} / \mathrm{d}, \mathrm{p}<0.001)$, used more often folate supplements $(100 \%$ versus $12 \%, p<0.001)$, had higher intakes of vitamin B6 (2.1 (3.4) mg/d versus $1.8(0.8) \mathrm{mg} / \mathrm{d}, \mathrm{p}<0.05)$, higher intakes of vitamin B12 (5.4 (8.0) $\mu \mathrm{g} / \mathrm{d}$ versus $4.3(2.1) \mu \mathrm{g} / \mathrm{d}, \mathrm{p}<0.05)$, and had higher plasma folate levels $(40.9 \pm$ $18.6 \mathrm{nmol} / \mathrm{L}$ versus $17.9 \pm 9.7 \mathrm{nmol} / \mathrm{L}, \mathrm{p}<0.001$ ) compared to women with an inadequate folate intake (Table 8.1). Nine women (13.6\%) had inadequate folate plasma levels. Eight of them did not meet the recommended dietary folate intake. Total median (IQR) folate intake was 713 (672) FE $\mu \mathrm{g} / \mathrm{d}$, of which 262 (102) FE $\mu \mathrm{g} / \mathrm{d}$ was from dietary sources (Table 8.2). Vegetables (25\%), bread and cereal products (22\%), dairy products $(10 \%)$, fruit $(10 \%)$, and oils and fats $(5 \%)$ were the main dietary sources of folate intake. Total folate intake (adjusted partial correlation coefficient $0.55, \mathrm{p}$ for trend $<0.001$ ) and folate from supplements (adjusted partial correlation coefficient $0.75, p$ for trend $<0.001$ ) were significantly positively associated with plasma folate levels. None of the dietary sources of folate was significantly associated with plasma folate. The linear dose-response curve for total folate intake and plasma folate level is shown in Figure $\mathbf{8 . 1}$ ( $p$ for non-linearity=0.69).

Vitamin D intake \& status

Twenty-two participants (33\%) had an adequate vitamin $D$ intake ( $\geq 10 \mu \mathrm{g} / \mathrm{d}$ ), while forty-four participants did not meet this recommendation. Women with an adequate vitamin $D$ intake had a significantly higher median (IQR) total vitamin D intake (13.0 (2.1) $\mu \mathrm{g} / \mathrm{d}$ versus $3.7(3.2) \mu \mathrm{g} / \mathrm{d}, \mathrm{p}<0.001$ ), used more often vitamin $D$ supplements $(100 \%$ versus $18 \%, p<0.001)$, and had higher serum $25(\mathrm{OH}) \mathrm{D}$ levels $(76 \pm 19 \mathrm{nmol} / \mathrm{L}$ versus $68 \pm 26 \mathrm{nmol} / \mathrm{L}, \mathrm{p}=0.16$ ) compared to women with an inadequate vitamin $D$ intake (Table 8.1). Fifteen women (22.7\%) had insufficient 25(OH)D serum levels, of which thirteen had dietary intakes below the recommendation. 
Table 8.1: Characteristics of the total study population $(n=66)$ and stratified by meeting the recommended intake of folate, vitamin D, and EPA + DHA for women in the preconception period according to the Health Council of the Netherlands.

\begin{tabular}{|c|c|c|c|c|c|c|c|}
\hline Characteristics & $\begin{array}{l}\text { Total } \\
\text { population } \\
(\mathrm{n}=66) \\
\end{array}$ & $\begin{array}{l}\text { Folate intake } \\
<680 \mathrm{FE} \mu \mathrm{g} / \mathrm{d} \\
(\mathrm{n}=33)\end{array}$ & $\begin{array}{l}\text { Folate intake } \\
\geq 680 \mathrm{FE} \mu \mathrm{g} / \mathrm{d} \\
(\mathrm{n}=33)\end{array}$ & $\begin{array}{l}\text { Vitamin D } \\
\text { intake } \\
<10 \mu \mathrm{g} / \mathrm{d} \\
(\mathrm{n}=44)\end{array}$ & $\begin{array}{l}\text { Vitamin D } \\
\text { intake } \\
\geq 10 \mu \mathrm{g} / \mathrm{d} \\
(\mathrm{n}=22)\end{array}$ & $\begin{array}{l}\text { EPA+DHA } \\
\text { intake } \\
<200 \mathrm{mg} / \mathrm{d} \\
(\mathrm{n}=37)\end{array}$ & $\begin{array}{l}\text { EPA+DHA } \\
\text { intake } \\
\geq 200 \mathrm{mg} / \mathrm{d} \\
(\mathrm{n}=29)\end{array}$ \\
\hline Maternal age (years) & $31.7 \pm 4.1$ & $31.2 \pm 4.7$ & $32.1 \pm 3.4$ & $31.0 \pm 4.0$ & $33.0 \pm 4.0$ & $31.7 \pm 4.5$ & $31.7 \pm 3.5$ \\
\hline $\mathrm{BMI}\left(\mathrm{kg} / \mathrm{m}^{2}\right)$ & $25.2 \pm 4.0$ & $26.1 \pm 4.4$ & $24.3 \pm 4.2$ & $25.3 \pm 4.5$ & $25.0 \pm 4.4$ & $24.9 \pm 4.6$ & $25.6 \pm 4.2$ \\
\hline Western ethnicity (\%) & 63 (95.5\%) & $30(90.9 \%)$ & $33(100 \%)$ & $42(95.5 \%)$ & 21 (95.5\%) & 34 (91.9\%) & 29 (100.0\%) \\
\hline $\begin{array}{l}\text { Marital status married } \\
\text { (\%) }\end{array}$ & $55(83.3 \%)$ & $27(81.8 \%)$ & $28(84.9 \%)$ & 37 (84.1\%) & $18(81.8 \%)$ & $32(86.5 \%)$ & $23(79.3 \%)$ \\
\hline Parity, $\geq 1$ child (\%) & $60(90.9 \%)$ & $29(87.9 \%)$ & $31(93.9 \%)$ & $39(88.6 \%)$ & $21(95.5 \%)$ & 34 (91.9\%) & $26(89.7 \%)$ \\
\hline \multicolumn{8}{|l|}{ Educational level (\%) ${ }^{1}$} \\
\hline Low & & 2 (6.1\%) & $1(2.6 \%)$ & & & & $1(3.5 \%)$ \\
\hline Interm & 3\%) & 12 & $10(30$ & & & & \\
\hline High & & $19(57$. & $22(66$. & & & & \\
\hline Smokers (\%) & $8(12.1 \%)$ & $5(15.2 \%)$ & $3(9.1 \%)$ & $6(13.6 \%)$ & 2 (9.1\%) & $4(10.8 \%)$ & $4(13.8 \%)$ \\
\hline Alcohol (g/d) & $0.9(4.1)$ & $0.5(4.1)$ & $0.9(3.6)$ & & $1.0(4.3)$ & & $1.8(3.9)^{*}$ \\
\hline $\begin{array}{l}\text { Blood sampli } \\
\text { December an }\end{array}$ & $10(15.2 \%)$ & $5(15.2 \%)$ & $5(15.2 \%)$ & 7 (15.9\%) & $3(13.6 \%)$ & $4(10.8 \%)$ & $6(20.7 \%)$ \\
\hline Energy (kJ) & $8424(2701)$ & 8152 (2888) & $8697(2058)$ & $8338(2752)$ & 8495 (2218) & 8152 (2376) & 8913 (2172) \\
\hline Total carbohydra & $45.4(5.8)$ & $44.9(6.6)$ & $46.0(6.3)$ & 45.4 (7.5) & $45.2(4.6)$ & 45.71 & 44.4 (7.7) \\
\hline Total protein $(\mathrm{E} \%)^{2}$ & $15.7(2.5)$ & $15.9(1.6)$ & $14.9(3.2)$ & $15.4(2.6)$ & $15.9(2.4)$ & $15.8(2.9)$ & $15.6 \pm 2.1$ \\
\hline Total fat $(\mathrm{E} \%)^{2}$ & 36.2 (5.9) & $36.2(5.0)$ & $36.3(5.4)$ & $36.2(6.6)$ & $36.4(5.0)$ & $35.1(5.3)$ & $37.1(6.3)$ \\
\hline Total folate (FE $\mu \mathrm{g}$ ) & $713(672)$ & $272(102)$ & $943(124)^{* *}$ & $323(657)$ & $898(145)^{* *}$ & $338(700)$ & $892(551)$ \\
\hline Folate supplement (\%) & $37(56.1 \%)$ & $4(12.1 \%)$ & $33(100 \%)^{* *}$ & $18(40.9 \%)$ & $19(86.4 \%)^{* *}$ & $16(43.2 \%)$ & $21(72.4 \%)^{*}$ \\
\hline Total vitamin D & $5.9(8.5)$ & $3.7(3.0)$ & $10.3(7.4)^{* *}$ & $3.7(3.2)$ & $13.0(2.1)^{* *}$ & $4.8(8.9)$ & $6.4(7.0)$ \\
\hline amin D supplement & $30(45.5 \%)$ & $7(21.2 \%)$ & $23(69.7 \%)^{* *}$ & $8(18.2 \%)$ & $\begin{array}{l}22 \\
(100.0 \%)^{* *}\end{array}$ & $15(40.5 \%)$ & $15(51.7 \%)$ \\
\hline Total EPA + DHA (mg) & $170(200)$ & $130(150)$ & $220(250)$ & $180(160)$ & $160(270)$ & $100(110)$ & $310(240)^{* *}$ \\
\hline $\begin{array}{l}\text { EPA+DHA supplement } \\
\text { (\%) }\end{array}$ & $3(4.6 \%)$ & $1(3.0 \%)$ & $2(6.1 \%)$ & $3(6.8 \%)$ & $0(0.0 \%)$ & $0(0.0 \%)$ & $3(10.3 \%)^{*}$ \\
\hline & $1.9(1.4)$ & & & & & & $1.9(1.2)$ \\
\hline Total vitamin B12 $(\mu \mathrm{g})$ & $4.6(3.3)$ & $4.3(2.1)$ & $5.4(8.0)^{*}$ & $4.4(2.3)$ & $5.6(9.8)$ & $4.1(2.2)$ & $5.8(4.7)^{* *}$ \\
\hline Plasma folate (nmol/L) & $29.4 \pm 18.7$ & $17.9 \pm 9.7$ & $40.9 \pm 18.6^{* *}$ & $26.9 \pm 18.4$ & $34.4 \pm 18.7$ & $26.9 \pm 15.5$ & $32.6 \pm 22.0$ \\
\hline $\begin{array}{l}\text { Insufficient plasma folate } \\
(<10 \mathrm{nmol} / \mathrm{L})\end{array}$ & $9(13.6 \%)$ & $8(24.2 \%)$ & $1(3.0 \%)^{*}$ & $8(18.2 \%)$ & $1(4.5 \%)$ & $4(10.8 \%)$ & $5(17.2 \%)$ \\
\hline Serum 25(OH)D (nmol/L) & $70.6 \pm 23.8$ & $65.7 \pm 26.2$ & $75.5 \pm 20.5$ & $67.6 \pm 25.7$ & $76.5 \pm 18.5$ & $68.6 \pm 22.9$ & $73.1 \pm 25.5$ \\
\hline $\begin{array}{l}\text { Insufficient serum } \\
25(\mathrm{OH}) \mathrm{D}(<50 \mathrm{nmol} / \mathrm{L})\end{array}$ & $15(22.7 \%)$ & $10(30.3 \%)$ & $5(15.2 \%)$ & $13(29.6 \%)$ & $2(9.1 \%)$ & $9(24.3 \%)$ & $6(20.7 \%)$ \\
\hline \multicolumn{8}{|l|}{ Plasma phospholipid } \\
\hline $\begin{array}{l}\text { EPA + DHA (g/100g } \\
\text { FAME) }\end{array}$ & $5.2 \pm 1.7$ & $5.0 \pm 1.6$ & $5.3 \pm 1.8$ & $5.2 \pm 1.8$ & $5.0 \pm 1.5$ & $4.6 \pm 1.6$ & $5.9 \pm 1.5^{* *}$ \\
\hline \multicolumn{8}{|c|}{$\begin{array}{l}\text { 25(OH)D 25-hydroxyvitamin D; EPA eicosapentaenoic acid; DHA docosahexaenoic acid; FAME Fatty Acid Methyl Esters. } \\
\text { Data are presented as mean } \pm \text { standard deviation, as median (interquartile range), or as } n(\%) \text {. Subgroups were created } \\
\text { based on the recommended intake of folate, vitamin D, and EPA + DHA for women in the preconception period according } \\
\text { to the Health Council of the Netherlands. } \\
\text { Independent t-tests, Chi-Square tests, and Mann Whitney U tests were performed between the group below and above } \\
\left.\text { the recommended nutrient intake of interest ( }{ }^{*} p \leq 0.05, * * p \leq 0.01\right) \text {. } \\
{ }^{1} \text { Low educational level: primary school, vocational or lower general secondary education; intermediate educational level: } \\
\text { higher secondary education or intermediate vocational training; high educational level: higher vocational education or } \\
\text { university. }\end{array}$} \\
\hline
\end{tabular}


Table 8.2: Absolute ( $\mu \mathrm{g} / \mathrm{d}$ ) and relative (\%) contribution of dietary sources to total dietary folate intake, Spearman's rank and partial correlation coefficients $(R)$ between folate intake and plasma folate levels, and adjusted means with $95 \%$ confidence intervals for plasma folate levels ( $\mathrm{nmol} / \mathrm{L}$ ) according to tertiles of folate intake $(\mu \mathrm{g} / \mathrm{d})$ among Dutch women with a pregnancy wish $(\mathrm{n}=66)$.

\begin{tabular}{|c|c|c|c|c|c|c|c|c|}
\hline & \multicolumn{2}{|c|}{ Contribution } & \multicolumn{2}{|c|}{ Correlation } & \multicolumn{3}{|c|}{ Adjusted means with $95 \% \mathrm{Cls}$} & \multirow{2}{*}{$\begin{array}{l}P \text { for } \\
\text { trend }\end{array}$} \\
\hline & $\mu \mathrm{g} / \mathrm{d}^{1}$ & $\%$ & $R^{2}$ & $\mathrm{R}^{3}$ & Tertile 1 & Tertile 2 & Tertile 3 & \\
\hline Total folate intake & $713(672)$ & & $0.58 * *$ & $0.55^{* *}$ & $\leq 294$ & 295-894 & $\geq 895$ & \\
\hline Plasma folate & & & & & $\begin{array}{c}17.6 \\
(10.6-24.7)\end{array}$ & $\begin{array}{c}28.6 \\
(21.7-35.4)\end{array}$ & $\begin{array}{c}42.0 \\
(34.8-49.2)\end{array}$ & $<.001$ \\
\hline Folate from supplements & $340(680)$ & & $0.68 * *$ & $0.67^{* *}$ & $\leq 0$ & $0.1-679$ & $\geq 680$ & \\
\hline Plasma folate & & & & & $\begin{array}{c}17.6 \\
(12.2-23.0)\end{array}$ & $\begin{array}{c}29.7 \\
(19.1-40.3)\end{array}$ & $\begin{array}{c}40.7 \\
(35.5-46.0)\end{array}$ & $<.001$ \\
\hline Total dietary folate & $262(102)$ & 100 & -0.08 & -0.20 & $\leq 223$ & 224-293 & $\geq 294$ & \\
\hline Plasma folate & & & & & $\begin{array}{c}31.4 \\
(25.5-37.3)\end{array}$ & $\begin{array}{c}30.2 \\
(24.5-35.9)\end{array}$ & $\begin{array}{c}26.5 \\
(20.6-32.5)\end{array}$ & 0.257 \\
\hline Folate from vegetables & $63.2(50.2)$ & 25 & 0.06 & 0.04 & $\leq 47.9$ & $48.0-83.3$ & $\geq 83.4$ & \\
\hline Plasma folate & & & & & $\begin{array}{c}30.1 \\
(24.6-35.6)\end{array}$ & $\begin{array}{c}25.2 \\
(19.8-30.7)\end{array}$ & $\begin{array}{c}33.1 \\
(27.6-38.6)\end{array}$ & 0.324 \\
\hline $\begin{array}{l}\text { Folate from bread and cereal } \\
\text { products } 5\end{array}$ & $57.5(39.3)$ & 22 & -0.10 & -0.19 & $\leq 46.0$ & $46.1-69.5$ & $\geq 69.6$ & \\
\hline Plasma folate & & & & & $\begin{array}{c}29.9 \\
(24.3-35.5)\end{array}$ & $\begin{array}{c}29.7 \\
(24.0-35.4)\end{array}$ & $\begin{array}{c}28.6 \\
(22.8-34.4)\end{array}$ & 0.763 \\
\hline \multirow{2}{*}{$\begin{array}{l}\text { Folate from dairy products } \\
\text { Plasma folate }\end{array}$} & $26.8(24.0)$ & 10 & 0.01 & -0.15 & $\leq 18.7$ & $18.8-34.0$ & $\geq 34.1$ & \\
\hline & & & & & $\begin{array}{c}32.6 \\
(26.6-38.6)\end{array}$ & $\begin{array}{c}27.3 \\
(21.8-32.9)\end{array}$ & $\begin{array}{c}28.5 \\
(22.2-34.8)\end{array}$ & 0.355 \\
\hline Folate from fruit & $25.6(22.4)$ & 10 & 0.13 & -0.14 & $\leq 17.6$ & $17.7-30.7$ & $\geq 30.8$ & \\
\hline Plasma folate & & & & & $\begin{array}{c}29.5 \\
(23.5-35.5)\end{array}$ & $\begin{array}{c}32.0 \\
(26.5-37.5)\end{array}$ & $\begin{array}{c}26.5 \\
(20.4-32.6)\end{array}$ & 0.502 \\
\hline Folate from oils and fats ${ }^{7}$ & $0.27(3.32)$ & 5 & -0.23 & -0.20 & $\leq 0.07$ & $0.08-0.63$ & $\geq 0.64$ & \\
\hline Plasma folate & & & & & $\begin{array}{c}32.0 \\
(26.4-37.7)\end{array}$ & $\begin{array}{c}30.7 \\
(25.0-36.4)\end{array}$ & $\begin{array}{c}25.4 \\
(19.7-31.2)\end{array}$ & 0.102 \\
\hline
\end{tabular}

Folate intake (FE $\mu \mathrm{g} / \mathrm{d}$ ), Plasma folate (nmol/L), * $\mathrm{p} \leq 0.05, * * \mathrm{p} \leq 0.01$.

${ }^{1}$ Median (IQR).

${ }^{2}$ Spearman's rank correlation with plasma folate $(\mathrm{nmol} / \mathrm{L})$ as dependent variable.

${ }_{3}^{3}$ Partial correlation with plasma folate $(\mathrm{nmol} / \mathrm{L})$ as dependent variable: adjusted for season of blood sampling, total energy intake (kJ), intake of total vitamin B6 and vitamin B12. For supplemental folate, additional adjustment was done for dietary folate intake; for dietary folate, additional adjustment was done for folate intake from supplement; for folate from top-5 dietary sources additional adjustment for folate intake from other selected dietary sources (i.e., vegetables, bread and cereal products, dairy products, fruit, and oils and fats) was done.

${ }^{4}$ Means were adjusted for the same covariates as for partial correlation, and calculated with ANCOVA.

5 includes bread, breakfast cereals, pasta, and rice.

${ }^{6}$ includes milk, yoghurt drinks, cheese, yoghurt, fromage frais, coffee creamer, and ice cream.

7 includes liquid, soft and hard cooking fats and margarine, and vegetable oils. 
Total median (IQR) vitamin D intake was 5.9 (8.5) $\mu \mathrm{g} / \mathrm{d}$, of which $3.3(2.0) \mu \mathrm{g} / \mathrm{d}$ was obtained from dietary sources (Table 8.3). Oils and fats (39\%) were the main contributors to total dietary vitamin $D$ intake. Fish intake was the second most important contributor (20\%). Furthermore, meat (14\%), eggs $(10 \%)$, and dairy products (5\%) belonged to the top-5 dietary sources of vitamin D intake. Total vitamin D intake (adjusted partial correlation coefficient $0.42, p$ for trend 0.04 ), vitamin $D$ from supplements (adjusted partial correlation coefficient $0.40, p$ for trend 0.006 ), total dietary vitamin $\mathrm{D}$ (adjusted partial correlation coefficient $0.30, \mathrm{p}$ for trend 0.001 ) and vitamin $\mathrm{D}$ from oils and fats (adjusted partial correlation coefficient $0.38, p$ for trend 0.02 ) were significantly positively associated with serum $25(\mathrm{OH}) \mathrm{D}$ levels. The dose-response between total vitamin $\mathrm{D}$ intake and serum $25(\mathrm{OH}) \mathrm{D}$ levels is shown in Figure 8.1 ( $p$ for linearity=0.17).

\section{EPA and DHA intake \& status}

Forty-four percent of the participants met the recommended intake of the n-3 fatty acids EPA+DHA $(\geq 200 \mathrm{mg} / \mathrm{d} ; \mathrm{n}=29)$; these women had a significantly higher median total EPA+DHA intake (310 (240) mg/d versus 100 (110) mg/d, $\mathrm{p}<0.001)$ compared with those who did not meet the recommendation (Table 8.1). Only three women used a supplement containing EPA+DHA. Plasma phospholipid EPA+DHA levels were significantly higher in participants with an adequate EPA+DHA intake than in participants with an inadequate intake $(5.9 \pm 1.5 \mathrm{~g} / 100 \mathrm{~g}$ FAME versus $4.6 \pm 1.6 \mathrm{~g} / 100 \mathrm{~g}$ FAME, $p=0.001$ ). Total median (IQR) EPA+DHA intake was $170(200) \mathrm{mg} / \mathrm{d}$, of which 165 (190) mg/d was from dietary sources (Table 8.4). Fish (69\%) was the main contributor to total dietary EPA+DHA intake, followed by meat (6\%) and eggs (4\%). Total EPA+DHA intake (adjusted partial correlation coefficient $0.67, p$ for trend 0.002), total dietary EPA+DHA (adjusted partial correlation coefficient 0.63, $p$ for trend 0.001), EPA+DHA from total fish intake (adjusted partial correlation coefficient $0.67, p$ for trend $<0.001$ ) and EPA+DHA from fatty fish (adjusted partial correlation coefficient 0.51, $p$ for trend $=0.001$ ) were significantly positively associated with plasma phospholipid EPA+DHA levels. Only three women took EPA+DHA containing supplements, therefore no $p$ for trend could be calculated, but EPA+DHA from supplements was significantly correlated with plasma phospholipid EPA+DHA levels (adjusted partial correlation coefficient $0.38, p=0.02$ ). Figure 8.1 shows the nonlinear dose-response curve between total EPA+DHA intake and plasma phospholipid EPA+DHA level, with the curve flattening at higher intake levels ( $p$ for non-linearity $=0.05$ ). 
Table 8.3: Absolute $(\mu \mathrm{g} / \mathrm{d})$ and relative (\%) contribution of dietary sources to total dietary vitamin $D$ intake, Spearman's rank and partial correlation coefficients $(R)$ between vitamin D intake and serum 25(OH)D levels, and adjusted means with $95 \%$ confidence intervals for serum $25(\mathrm{OH}) \mathrm{D}$ levels $(\mathrm{nmol} / \mathrm{L})$ according to tertiles of vitamin D intake $(\mu \mathrm{g} / \mathrm{d})$ among Dutch women with a pregnancy wish $(\mathrm{n}=66)$.

\begin{tabular}{|c|c|c|c|c|c|c|c|c|}
\hline & \multicolumn{2}{|c|}{ Contribution } & \multicolumn{2}{|c|}{ Correlation } & \multicolumn{3}{|c|}{ Adjusted means with $95 \% \mathrm{Cls}$} & \multirow{2}{*}{$\begin{array}{l}\text { P for } \\
\text { trend }\end{array}$} \\
\hline & $\mu \mathrm{g} / \mathrm{d}^{1}$ & $\%$ & $\mathrm{R}^{2}$ & $\mathrm{R}^{3}$ & Tertile 1 & Tertile 2 & Tertile 3 & \\
\hline Total vitamin D & $5.9(8.5)$ & & $0.32^{* *}$ & $0.42 * *$ & $\leq 3.6$ & $3.7-10.2$ & $\geq 10.3$ & \\
\hline Serum $25(\mathrm{OH}) \mathrm{D}$ & & & & & $\begin{array}{c}62.5 \\
(52.6-72.5)\end{array}$ & $\begin{array}{c}71.2 \\
(61.5-80.9)\end{array}$ & $\begin{array}{c}78.0 \\
(68.4-87.5)\end{array}$ & 0.04 \\
\hline $\begin{array}{l}\text { Vitamin D from } \\
\text { supplements }\end{array}$ & $0(7.5)$ & & $0.30 *$ & $0.40^{* *}$ & $\leq 0$ & $0.1-4.9$ & $\geq 5.0$ & \\
\hline Serum 25(OH)D & & & & & $\begin{array}{c}64.5 \\
(57.6-71.5)\end{array}$ & $\begin{array}{c}55.6 \\
(24.8-86.3)\end{array}$ & $\begin{array}{c}79.4 \\
(71.5-87.3)\end{array}$ & 0.006 \\
\hline Total dietary vitamin D & $3.3(2.0)$ & 100 & 0.12 & $0.30 *$ & $\leq 2.8$ & 2.9-3.8 & $\geq 3.9$ & \\
\hline Serum 25(OH)D & & & & & $\begin{array}{c}57.4 \\
(48.2-66.6)\end{array}$ & $\begin{array}{c}71.5 \\
(62.8-80.2)\end{array}$ & $\begin{array}{c}82.8 \\
(72.9-92.6)\end{array}$ & 0.001 \\
\hline $\begin{array}{l}\text { Vitamin D from oils and } \\
\text { fats }{ }^{5}\end{array}$ & $1.1(2.0)$ & 39 & 0.20 & $0.38^{* *}$ & $\leq 0.6$ & $0.7-2.0$ & $\geq 2.1$ & \\
\hline Serum 25(OH)D & & & & & $\begin{array}{c}60.6 \\
(50.2-71.1)\end{array}$ & $\begin{array}{c}69.6 \\
(60.8-78.3)\end{array}$ & $\begin{array}{c}80.7 \\
(70.1-91.3)\end{array}$ & 0.02 \\
\hline Vitamin D from fish & $0.53(0.76)$ & 20 & -0.04 & 0.18 & $\leq 0.2$ & $0.3-0.7$ & $\geq 0.8$ & \\
\hline Serum 25(OH)D & & & & & $\begin{array}{c}63.3 \\
(53.5-73.2)\end{array}$ & $\begin{array}{c}73.8 \\
(65-82.6)\end{array}$ & $\begin{array}{c}74.1 \\
(64.8-83.3)\end{array}$ & 0.18 \\
\hline $\begin{array}{l}\text { Vitamin D from meat } \\
\text { Serum } 25(\mathrm{OH}) \mathrm{D}\end{array}$ & $0.43(0.37)$ & 14 & 0.09 & 0.03 & $\begin{array}{c}\leq 0.3 \\
69.2 \\
(59.6-78.8)\end{array}$ & $\begin{array}{c}0.4-0.6 \\
69.5 \\
(59.9-79)\end{array}$ & $\begin{array}{c}\geq 0.7 \\
73.0 \\
(63.1-83.0)\end{array}$ & 0.43 \\
\hline $\begin{array}{l}\text { Vitamin D from egg } \\
\text { Serum } 25(\mathrm{OH}) \mathrm{D}\end{array}$ & $0.23(0.35)$ & 10 & -0.14 & -0.07 & $\begin{array}{c}\leq 0.1 \\
72.0 \\
(62.1-81.9)\end{array}$ & $\begin{array}{c}0.2-0.3 \\
67.5 \\
(57.4-77.6)\end{array}$ & $\begin{array}{c}\geq 0.4 \\
71.8 \\
(61.7-81.9)\end{array}$ & 0.96 \\
\hline $\begin{array}{l}\text { Vitamin D from dairy } \\
\text { products }^{6}\end{array}$ & $0.12(0.11)$ & 5 & -0.06 & 0.13 & $\leq 0.01$ & $0.02-0.10$ & $\geq 0.11$ & \\
\hline Serum $25(\mathrm{OH}) \mathrm{D}$ & & & & & $\begin{array}{c}74.0 \\
(65.0-83.0)\end{array}$ & $\begin{array}{c}63.7 \\
(54.0-73.4)\end{array}$ & $\begin{array}{c}73.5 \\
(63.8-83.2)\end{array}$ & 0.11 \\
\hline
\end{tabular}

25(OH)D 25-hydroxyvitamin D.

Vitamin $D$ intake $(\mu \mathrm{g} / \mathrm{d})$, and serum $25(\mathrm{OH}) \mathrm{D}(\mathrm{nmol} / \mathrm{L}),{ }^{*} \mathrm{p} \leq 0.05, * * \mathrm{p} \leq 0.01$.

${ }^{1}$ Median (IQR).

2 Spearman's rank correlation with serum $25(\mathrm{OH}) \mathrm{D}(\mathrm{nmol} / \mathrm{L})$ as dependent variable.

${ }^{3}$ Partial correlation with serum 25(OH)D as dependent variable: adjusted for season of blood sampling, education level (low/intermediate/high), BMI ( $\mathrm{kg} / \mathrm{m}^{2}$ ) and energy intake (kJ). For supplemental vitamin D additionally adjusted for dietary vitamin $D$ intake; for dietary vitamin $D$ additionally adjusted for vitamin $D$ intake from supplement; for vitamin $D$ from top5 dietary sources additional adjustment for vitamin $\mathrm{D}$ intake from other selected dietary sources (i.e., oils and fats, fish, meat, egg, and dairy products).

${ }^{4}$ Means were adjusted for the same covariates as for partial correlation, and calculated with ANCOVA.

5 includes liquid, soft and hard cooking fats and margarine, and vegetable oils.

6 includes milk, yoghurt drinks, cheese, yoghurt, fromage frais, coffee creamer, and ice cream. 
Table 8.4: Absolute $(\mathrm{mg} / \mathrm{d})$ and relative $(\%)$ contribution of dietary sources to total EPA and DHA intake, Spearman's rank and partial correlation coefficients between EPA and DHA intake and plasma phospholipid EPA and DHA, and adjusted means with 95\% confidence intervals for plasma phospholipid EPA and DHA (g/100g FAME) according to tertiles of EPA and DHA intake $(\mathrm{mg} / \mathrm{d})$ among Dutch women with a pregnancy wish $(n=66)$.

\begin{tabular}{|c|c|c|c|c|c|c|c|c|}
\hline & \multicolumn{2}{|c|}{ Contribution } & \multicolumn{2}{|c|}{ Correlation } & \multicolumn{3}{|c|}{ Adjusted means with $95 \% \mathrm{Cls}$} & \multirow{2}{*}{$\begin{array}{l}\text { P for } \\
\text { trend }\end{array}$} \\
\hline & $\mathrm{mg} / \mathrm{d}^{1}$ & $\%$ & $\mathrm{R}^{2}$ & $\mathrm{R}^{3}$ & Tertile 1 & Tertile 2 & Tertile 3 & \\
\hline Total EPA + DHA intake & $170(200)$ & & $0.63^{* *}$ & $0.67^{* *}$ & $<100$ & $100-240$ & $>240$ & \\
\hline PPL g/100g FAME & & & & & $\begin{array}{c}4.1 \\
(3.4-4.8)\end{array}$ & $\begin{array}{c}5.3 \\
(4.6-6.0)\end{array}$ & $\begin{array}{c}6.0 \\
(5.4-6.7)\end{array}$ & 0.002 \\
\hline EPA + DHA from supplements & $0(0)$ & & 0.24 & $0.38^{*}$ & 0 & $1-500$ & & \\
\hline PPL g/100g FAME & & & & & $\begin{array}{c}5.0 \\
(4.6-5.4)\end{array}$ & $\begin{array}{c}7.9 \\
(6.1-9.7)\end{array}$ & $-\mathrm{a}$ & $-a$ \\
\hline Total dietary EPA + DHA & 165 (190) & 100 & $0.59 * *$ & $0.63 * *$ & $<100$ & $100-230$ & $>230$ & \\
\hline PPL g/100g FAME & & & & & $\begin{array}{c}4.2 \\
(3.5-4.8)\end{array}$ & $\begin{array}{c}5.5 \\
(4.8-6.2)\end{array}$ & $\begin{array}{c}5.9 \\
(5.2-6.5)\end{array}$ & 0.001 \\
\hline$E P A+D H A$ from fish & $135(190)$ & 69 & $0.60 * *$ & $0.67 * *$ & $<70$ & $70-190$ & $>190$ & \\
\hline PPL g/100g FAME & & & & & $\begin{array}{c}3.8 \\
(3.2-4.4)\end{array}$ & $\begin{array}{c}5.9 \\
(5.2-6.5)\end{array}$ & $\begin{array}{c}6.0 \\
(5.4-6.5)\end{array}$ & $<.001$ \\
\hline EPA + DHA from fatty fish & $105(170)$ & 46 & $0.60^{* *}$ & $0.51^{* *}$ & $<20$ & $20-160$ & $>160$ & \\
\hline PPL g/100g FAME & & & & & $\begin{array}{c}4.4 \\
(3.7-5)\end{array}$ & $\begin{array}{c}5.4 \\
(4.7-6)\end{array}$ & $\begin{array}{c}5.7 \\
(5-6.4)\end{array}$ & 0.009 \\
\hline$E P A+D H A$ from lean fish & $25(40)$ & 18 & 0.22 & 0.10 & $<10$ & $10-30$ & $>30$ & \\
\hline PPL g/100g FAME & & & & & $\begin{array}{c}4.9 \\
(4.2-5.5)\end{array}$ & $\begin{array}{c}5.3 \\
(4.7-6.0)\end{array}$ & $\begin{array}{c}5.3 \\
(4.6-6.0)\end{array}$ & 0.408 \\
\hline$E P A+D H A$ from shell fish & $0(10)$ & 5 & $0.32 *$ & 0.24 & 0 & $1-140$ & & \\
\hline PPL g/100g FAME & & & & & $\begin{array}{c}4.9 \\
(4.4-5.3)\end{array}$ & $\begin{array}{c}6.0 \\
(5.2-6.8)\end{array}$ & $-a$ & $-a$ \\
\hline$E P A+D H A$ from meat & $10(10)$ & 6 & -0.06 & $-0.30 *$ & 0 & $1-30$ & & \\
\hline PPL g/100g FAME & & & & & $\begin{array}{c}5.6 \\
(5-6.1)\end{array}$ & $\begin{array}{c}4.8 \\
(4.3-5.3)\end{array}$ & $-a$ & $-a$ \\
\hline$E P A+D H A$ from egg & $0(10)$ & 4 & 0.07 & -0.00 & 0 & $1-50$ & & \\
\hline PPL g/100g FAME & & & & & $\begin{array}{c}5.1 \\
(4.6-5.5)\end{array}$ & $\begin{array}{c}5.3 \\
(4.7-5.9)\end{array}$ & $-a^{a}$ & $-a^{2}$ \\
\hline
\end{tabular}

EPA eicosapentaenoic acid; DHA docosahexaenoic acid; FAME fatty acid methyl esters; PPL plasma phospholipid. EPA + DHA intake (mg/d), and plasma phospholipid EPA+DHA (g/100g FAME), $* \mathrm{p} \leq 0.05, * * \mathrm{p} \leq 0.01$.

${ }^{1}$ Median (IQR).

2 Spearman's rank correlation with plasma phospholipid EPA + DHA (g/100g FAME) as dependent variable.

3 Partial correlation with plasma phospholipid EPA + DHA (g/100g FAME) as dependent variable: adjusted for season of blood sampling, education level (low/intermediate/high), BMI $\left(\mathrm{kg} / \mathrm{m}^{2}\right)$ and energy intake ( $\mathrm{kJ}$ ). For supplemental EPA+DHA additionally adjusted for dietary EPA+DHA intake; for dietary EPA+DHA additionally adjusted for EPA+DHA intake from supplement; for EPA+DHA from top-5 dietary sources additional adjustment for EPA+DHA intake from other selected dietary sources (i.e., fish (fatty, lean and shell fish), meat, and egg).

${ }^{4}$ Means were adjusted for the same covariates as for partial correlation, and calculated with ANCOVA.

a Due to the low amount of EPA+DHA in lean fish, shellfish, meat, and egg, two groups were made instead of tertiles and no $\mathrm{p}$ for trend could be calculated. 

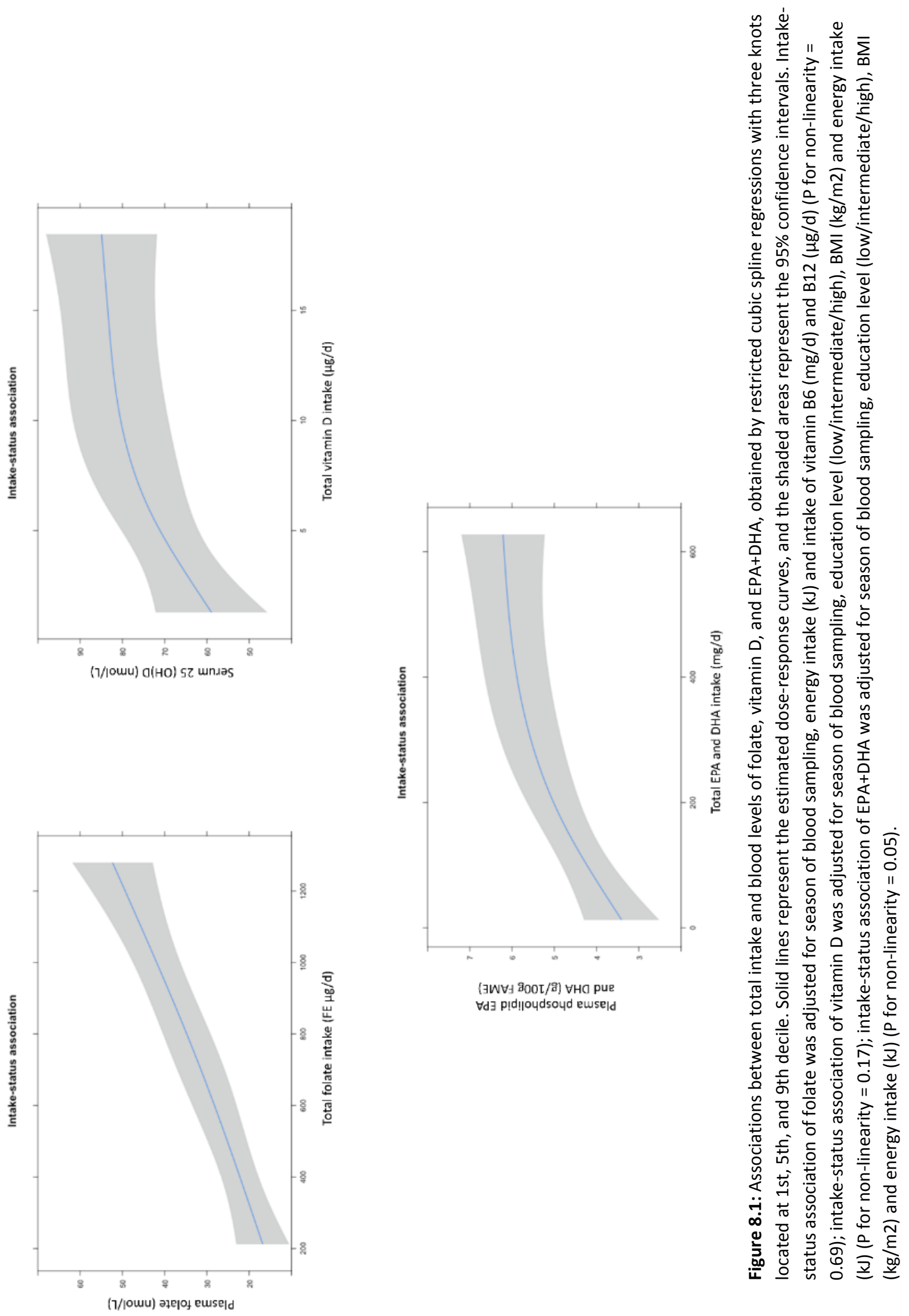


\section{Discussion}

In our cross-sectional study in 66 Dutch women aged $18-40$ years who wished to become pregnant, $50 \%$ had an inadequate folate intake $(<680 \mathrm{FE} \mu \mathrm{g} / \mathrm{d}), 67 \% \mathrm{had}$ an inadequate vitamin $\mathrm{D}$ intake $(<10$ $\mu \mathrm{g} / \mathrm{d})$, and $56 \%$ had an inadequate EPA+DHA intake $(<200 \mathrm{mg} / \mathrm{d})$ according to the recommendations given by the Health Council of the Netherlands. Dietary intakes of folate, vitamin D and EPA+DHA were significantly positively associated with their blood levels. However, $14 \%$ of the women had an inadequate folate status and $23 \%$ an inadequate vitamin D status. We observed significant associations between folate intake from supplements with plasma folate levels, whereas dietary folate intake was not associated with folate status markers. Oils and fats were the main contributors to total dietary vitamin D intake followed by fish. Significant vitamin D intake-status associations were observed for total vitamin D intake, vitamin D from supplements, dietary vitamin $D$ and vitamin $D$ from oils and fats with serum 25(OH)D. Fish, mainly fatty fish, was the most important contributor to dietary EPA+DHA intake. In line with this, significant associations were observed between total EPA+DHA intake, total dietary EPA+DHA intake, and EPA+DHA obtained from (fatty) fish with plasma phospholipid EPA+DHA.

\section{Folate intake}

Despite the fact that all women in this study wished to become pregnant within a year, only $50 \%$ of the women used a folate-containing supplement and met the recommended folate intake of $680 \mathrm{FE}$ $\mu \mathrm{g} / \mathrm{d}$. This is in line with previous research carried out among Dutch pregnant women that reported that $31 \%$ of women with a lower socioeconomic status and $63 \%$ of the women with a higher socioeconomic status used a folic acid supplement prior to conception [28].Folic acid supplement use in the preconception period reported in Australia was comparable [29, 30]. Other studies reporting supplement use during pregnancy found up to $95 \%$ of pregnant women using supplements, with multivitamin supplements and folic acid supplements were the most commonly reported [31-33]. Of the total folate intake reported (713 (672) FE $\mu \mathrm{g} / \mathrm{d}$ ), only 262 (102) $\mu \mathrm{g} / \mathrm{d}$ was from dietary sources. This highlights the substantial contribution of folate from supplements to total folate intake in this population. The observed dietary folate intake is comparable to results of the Dutch National Food Consumption Survey (DNFCS) 2007-2010, where the dietary folate intake was 216 (92) $\mu \mathrm{g} / \mathrm{d}$ for women aged 19-30 years and $242(102) \mu \mathrm{g} / \mathrm{d}$ for women aged 31-50 years [34]. The strong association between total folate intake and plasma folate levels as observed in our study population is supported by a metaanalysis, using data from mostly non-pregnant and non-lactating women of childbearing age, reporting a $47 \%$ increase in plasma folate levels when doubling total folate intake [35]. Vegetables and bread and cereal products were the main contributors to dietary folate intake in our population, which is in line with a study in healthy Norwegian women aged $47-49$ years and 71-74 years [36]. However, in this study, vegetables, fruit, and orange juice intake were significantly associated with plasma folate levels, 
whereas we did not observe this association. This may be explained by our specific population of women who wished to become pregnant and consequently included a larger proportion of supplement users, which resulted in a relative low contribution of dietary folate to total folate intake in our study.

\section{Vitamin D intake}

One-third of the women in our population had an adequate vitamin D intake (i.e. $\geq 10 \mu \mathrm{g}$ ) and all these women used a vitamin D supplement. Median total vitamin D intake of women with an inadequate vitamin D intake (5.9 (8.5) $\mathrm{\mu g} / \mathrm{d}$ ) was higher compared to results of the DNFCS 2007-2010, which estimated a median intake of 2.9 (1.9) $\mu \mathrm{g} / \mathrm{d}$ for women aged 19-30 years and 3.3 (2.3) $\mu \mathrm{g} / \mathrm{d}$ for women aged 31-50 years [34]. Of the total vitamin D intake reported in our study, $3.3(2.0) \mu g / d$ was from dietary sources. Oils and fats were the most important dietary sources contributing to dietary vitamin D intake, followed by fish, meat, eggs, and dairy products, which is in line with a recent study in Dutch older adults [37]. Furthermore, we observed significant associations between tertiles of total vitamin D intake, total dietary vitamin $D$ intake, vitamin $D$ from supplements, and vitamin $D$ intake from fats and oils with serum 25(OH)D levels, which is again in agreement with the recent study in Dutch older adults. Thus, even though sunlight is the most important source of vitamin D [15], we and Vaes (2016) showed that both dietary and supplemental vitamin D may have a substantial impact on serum $25(\mathrm{OH}) \mathrm{D}$ levels. This is supported by a recent meta-analysis which observed that the intake of $\pm 300 \mathrm{~g}$ fish per week over a period of at least four weeks is associated with an increase in 25(OH)D levels [38]. Although fish intake was the second contributor in our population, we did not observe a significant association between fish intake and 25(OH)D levels. The difference between the meta-analysis and our study may relate to the fact that the median intake of $\pm 100 \mathrm{~g}$ fish per week in our study was substantially lower than the intake levels of $300 \mathrm{~g}$ fish per week as suggested in the meta-analysis [38]. Regarding vitamin D supplement use, $45 \%$ of the women in our study used a vitamin D supplement in the preconception period, which is high compared to a study in Australia, where only fourteen percent of the participants, also mainly high educated, used a vitamin D supplement during the three months prior to conception [29]. Furthermore, a pregnancy multivitamin supplement commonly used in our cohort contained both folate and vitamin $\mathrm{D}$, which may explain the higher vitamin $\mathrm{D}$ supplement use in our population. In addition, it explains why women with an adequate vitamin D intake, also had an adequate folate intake. Sixty-seven percent of the women in this population had an inadequate vitamin D intake, of which 13 women (30\%) had serum 25(OH)D levels below $50 \mathrm{nmol} / \mathrm{L}$. In the group with adequate vitamin D intake, only two out of 22 women (9\%) had serum 25(OH)D levels below 50 $\mathrm{nmol} / \mathrm{L}$. This effect was more pronounced in the winter season, and illustrates the importance of meeting the vitamin $D$ recommendation, especially in the winter. 


\section{EPA and DHA intake}

Less than half of the women in our study met the recommended $200 \mathrm{mg}$ daily intake of EPA+DHA. However, the median (IQR) intake of $170(200) \mathrm{mg} / \mathrm{d}$ in our study was higher than the intake of EPA+DHA in the general population with a median intake of $75(92) \mathrm{mg} / \mathrm{d}$ for women aged 19-30 years and 89 (106) mg/d for women aged 31-50 years [34]. Fish, and especially fatty fish, was the main contributor to dietary EPA+DHA intake in our study population. We observed strong significant correlations of total EPA+DHA intake, total dietary EPA+DHA intake, and EPA+DHA obtained from (fatty) fish with plasma phospholipid EPA+DHA. This is in line with a study among adult Swedish women showing comparable strong correlations between dietary intake of EPA+DHA and EPA+DHA plasma phospholipids [39]. Only three women in our study (5\%) used a supplement containing EPA+DHA, which is similar to the data of the DNFCS 2007-2010 [34] and to a cohort in Australia where 9\% of the women used a fish oil supplement during the three months prior to conception [29]. The women in our study who took EPA+DHA containing supplements had higher plasma phospholipid EPA+DHA plasma levels, adjusted for dietary EPA+DHA intake, than the women who did not take a supplement, indicating that for women who cannot or do not want to consume fish, EPA+DHA containing supplements can be helpful to achieve recommended intakes and increase EPA+DHA levels.

\section{Supplement use}

As demonstrated in our study, supplementation can help in achieving adequate intake levels and consequently achieve adequate blood levels of folate and vitamin D. However, some considerations should be taken into account regarding supplement use. Women who take prenatal supplements are often women with a higher educational level, who, in general, already achieve higher intakes of micronutrients $[40,41]$. Health policies encouraging supplement use might not reach the women with the lowest intake levels, but increase supplement consumption in health-conscious women with already adequate intake levels, who are consequently at risk of overconsumption [31]. This might especially occur in countries with mandatory fortification of bread, for example, with folate, such as in the United States, United Kingdom and Australia. It should also be noted that the WHO advises against using supplements, other than folic acid, vitamin $D$ and iron in the preconception period or during pregnancy when not deficient, including multivitamins as there has been no proven additional benefit of other supplements and may lead to overconsumption of specific micronutrients [42].

\section{Study limitations and strengths}

Before heading to the conclusion, some limitations and strengths of this study need to be discussed. First of all, the sample size of this study was relatively small $(n=66)$. However, significant correlations and associations were observed suggesting that study power does not seem to be an important limitation. Besides that, median (IQR) vitamin D intake from dietary sources was in line with the values 
described in the study of Vaes (2016) ( $n=595)$, indicating that our sample size was large enough to provide robust vitamin $D$ intake estimates. Secondly, our FFQ was not validated for vitamin $D$ and the n-3 fatty acids EPA and DHA, but was an updated version of an FFQ which was validated for dietary intake of energy, macronutrients, and B-vitamins in Dutch women of reproductive age [20-22]. However, the FFQ was designed to capture energy intake (i.e. an extensive FFQ including a large variety of food items) and included food items covering the most important dietary sources of vitamin $D$ and EPA+DHA (e.g. fat and oils, fish, meat, and dairy). Nevertheless, measurement errors resulting from aggregation of food-items in an FFQ, under- or over reporting, recall bias, seasonal influence and thus biased estimates associated with self-report methods cannot be excluded [21]. Thirdly, our study population consisted of mostly highly educated women with a Western ethnicity, with an oversampling of women with a high risk of GDM. Our results may therefore be limited in generalizability to other study populations with respect to reported dietary intake estimates and prevalence of inadequacy. However, intake-status associations are less likely to be influenced by education and were adjusted for both educational level and BMI (most important GDM risk factor).

Major strengths of this study include availability of blood samples of women in the preconception period and information on women's total nutrient intake, including both diet and supplement use, enabling us to examine intake-status associations. Because the FFQ covered dietary intake of the previous month, appropriate blood biomarkers were used, as plasma folate, serum 25(OH)D, and plasma phospholipid EPA+DHA levels were considered indicators of recent dietary intake [35, 43, 44]. Furthermore, potential relevant covariates appropriate to specific nutrient intakes were used in statistical analyses to reduce the risk of confounding.

\section{Conclusions}

Results of our study showed that even among highly educated women who wanted to become pregnant a large proportion did not meet recommendations regarding folate, vitamin D and EPA+DHA intake. Significant associations were found between total folate, total vitamin D and total EPA+DHA intake and their blood levels. Women with modest inadequate vitamin D and EPA+DHA intake may obtain an adequate intake by an increased consumption of fats and oils and fish. In this population, supplement use contributed substantially to total folate and vitamin D intake and status levels. Promotion of fish intake and folic acid and vitamin D supplement use of for women wish to become pregnant is necessary, since intake of the top- 5 dietary sources, covering at least $80 \%$ of dietary intakes of these micronutrients, cannot suffice the nutritional requirements in most women to obtain an adequate nutritional status in the preconception period. Results of our study contribute to the current 


\section{Chapter 8}

scientific evidence as data on intake of folate, vitamin D, and EPA+DHA from different sources and data on nutrient status is very limited, especially among women who wish to become pregnant.

Acknowledgements: We thank all participants for their time and contributions, and the research staff and students for their help in this study. Furthermore, we would like to thank the department of Gynaecology and Obstetrics and the department of Internal Medicine of hospitals Gelderse Vallei, Ede; Rijnstate, Arnhem; and Slingeland, Doetinchem for their help in the recruitment of participants.

Funding: This work was supported by the Division of Human Nutrition, Wageningen University and the Clinical Chemistry and Haematology Laboratory, Hospital Gelderse Vallei Ede (biochemical analyses).

Conflict of interest: The authors declare that they have no conflicts of interest. 


\section{References}

1. Abu-Saad, K. and D. Fraser, Maternal nutrition and birth outcomes. Epidemiol. Rev., 2010. 32: p. 5-25.

2. Emmett, P.M., L.R. Jones, and J. Golding, Pregnancy diet and associated outcomes in the Avon Longitudinal Study of Parents and Children. Nutr. Rev., 2015. 73 Suppl 3: p. 154-74.

3. Langley-Evans, S.C., Nutrition in early life and the programming of adult disease: a review. J. Hum. Nutr. Diet., 2015. 28 Suppl 1: p. 1-14.

4. Parisi, F., A. Laoreti, and I. Cetin, Multiple micronutrient needs in pregnancy in industrialized countries. Ann. Nutr. Metab., 2014. 65(1): p. 13-21.

5. Huijgen, N.A., M.E. van de Kamp, J.M. Twigt, et al., The Preconception Dietary Risk score; a simple tool to assess an inadequate habitual diet for clinical practice. e-SPEN Journal, 2014. 9(1): p. e13-e19.

6. Health Council of the Netherlands, Preconception care: a good beginning. Publication no. 2007/19, 2007: p. The Hague: Health Council of the Netherlands.

7. $\quad$ van der Meer, I.M., N.S. Karamali, A.J.P. Boeke, et al., High prevalence of vitamin $D$ deficiency in pregnant nonWestern women in The Hague, Netherlands. The American journal of clinical nutrition, 2006. 84(2): p. 350-353.

8. Lumley, J., L. Watson, M. Watson, et al., Periconceptional supplementation with folate and/or multivitamins for preventing neural tube defects. The Cochrane Library, 2001.

9. Bodnar, L.M., J.M. Catov, H.N. Simhan, et al., Maternal vitamin D deficiency increases the risk of preeclampsia. The Journal of Clinical Endocrinology and Metabolism, 2007. 92(9): p. 3517-3522.

10. Zhang, C., C. Qiu, F.B. Hu, et al., Maternal plasma 25-hydroxyvitamin D concentrations and the risk for gestational diabetes mellitus. PLoS One, 2008. 3(11): p. e3753.

11. Morley, R., J. Carlin, J. Pasco, et al., Maternal 25-hydroxyvitamin D concentration and offspring birth size: effect modification by infant VDR genotype. Eur. J. Clin. Nutr., 2009. 63(6): p. 802-804.

12. Javaid, M., S. Crozier, N. Harvey, et al., Maternal vitamin D status during pregnancy and childhood bone mass at age 9 years: a longitudinal study. The Lancet, 2006. 367(9504): p. 36-43.

13. Camargo, C.A., S.L. Rifas-Shiman, A.A. Litonjua, et al., Maternal intake of vitamin D during pregnancy and risk of recurrent wheeze in children at $3 y$ of age. The American Journal of Clinical Nutrition, 2007. 85(3): p. 788-795.

14. Devereux, G., A.A. Litonjua, S.W. Turner, et al., Maternal vitamin D intake during pregnancy and early childhood wheezing. The American Journal of Clinical Nutrition, 2007. 85(3): p. 853-859.

15. Brouwer-Brolsma, E.M., A.M. Vaes, N.L. van der Zwaluw, et al., Relative importance of summer sun exposure, vitamin D intake, and genes to vitamin D status in Dutch older adults: The B-PROOF study. The Journal of steroid biochemistry and molecular biology, 2016. 164: p. 168-176.

16. Health Council of the Netherlands, Evaluation of the dietary reference values for vitamin D. Publication no. 2008/15E, 2012: p. The Hague: Health Council of the Netherlands.

17. Uauy, R. and A.D. Dangour, Nutrition in brain development and aging: role of essential fatty acids. Nutr. Rev., 2006. 64(5 Pt 2): p. S24-33; discussion S72-91.

18. Health Council of the Netherlands, Richtlijnen goede voeding 2015 [in Dutch] Publication no. 2015/24, 2015: p. The Hague: Health Council of the Netherlands.

19. Netherlands Nutrition Centre, Richtlijnen Schijf van Vijf 2016, Netherlands Nutrition Centre: The Hague.

20. Verkleij-Hagoort, A.C., J.H. de Vries, M.P. Stegers, et al., Validation of the assessment of folate and vitamin B12 intake in women of reproductive age: the method of triads. Eur. J. Clin. Nutr., 2007. 61(5): p. 610-615.

21. Siebelink, E., A. Geelen, and J.H. de Vries, Self-reported energy intake by FFQ compared with actual energy intake to maintain body weight in 516 adults. Br. J. Nutr., 2011. 106(02): p. 274-281.

22. Streppel, M.T., J.H. de Vries, S. Meijboom, et al., Relative validity of the food frequency questionnaire used to assess dietary intake in the Leiden Longevity Study. Nutr. J., 2013. 12: p. 75.

23. NEVO-tabel, NEVO-tabel Voedingscentrum. 2006.

24. Institute of Medicine, Dietary Reference Intakes for Thiamin, Riboflavin, Niacin, Vitamin B6, Folate, Vitamin B12, Pantothenic Acid, Biotin, and Choline. 1998: Washington, D.C.

25. Health Council of the Netherlands, Towards an optimal use of folic acid. Publication no. 2008/02E, 2008: p. The Hague: Health Council of the Netherlands.

26. Health Council of the Netherlands, Towards an adequate intake of vitamin D. Publication no. 2008/15E, 2008: p. The Hague: Health Council of the Netherlands.

27. Glatz, J., A. Soffers, and M.B. Katan, Fatty acid composition of serum cholesteryl esters and erythrocyte membranes as indicators of linoleic acid intake in man. The American journal of clinical nutrition, 1989. 49(2): p. 269-276.

28. de Walle, H.E. and L.T. de Jong-van den Berg, Ten years after the Dutch public health campaign on folic acid: the continuing challenge. Eur. J. Clin. Pharmacol., 2008. 64(5): p. 539-43.

29. Livock, M., P.J. Anderson, S. Lewis, et al., Maternal micronutrient consumption periconceptionally and during pregnancy: a prospective cohort study. Public Health Nutr., 2017: p. 1-11.

30. McKenna, E., A. Hure, A. Perkins, et al., Dietary Supplement Use during Preconception: The Australian Longitudinal Study on Women's Health. Nutrients, 2017. 9(10).

31. Dubois, L., M. Diasparra, B. Bedard, et al., Adequacy of nutritional intake from food and supplements in a cohort of pregnant women in Quebec, Canada: the 3D Cohort Study (Design, Develop, Discover). Am. J. Clin. Nutr., 2017. 106(2): p. 541-548. 
32. Arkkola, T., U. Uusitalo, M. Pietikainen, et al., Dietary intake and use of dietary supplements in relation to demographic variables among pregnant Finnish women. Br. J. Nutr., 2006. 96(5): p. 913-20.

33. Gomez, M.F., C.J. Field, D.L. Olstad, et al., Use of micronutrient supplements among pregnant women in Alberta: results from the Alberta Pregnancy Outcomes and Nutrition (APrON) cohort. Matern. Child Nutr., 2015. 11(4): p. 497-510.

34. van Rossum, C.T.M., H.P. Fransen, et al., Dutch National Food Consumption Survey 2007-2010: Diet of children and adults aged 7 to 69 years. RIVM rapport 350050006, 2011: p. Bilthoven, National Institue for Public Health and the Environment.

35. Berti, C., K. Fekete, C. Dullemeijer, et al., Folate intake and markers of folate status in women of reproductive age, pregnant and lactating women: a meta-analysis. J. Nutr. Metab., 2012. 2012.

36. Brevik, A., S.E. Vollset, G.S. Tell, et al., Plasma concentration of folate as a biomarker for the intake of fruit and vegetables: the Hordaland Homocysteine Study. The American journal of clinical nutrition, 2005. 81(2): p. 434-439.

37. Vaes, A., E. Brouwer-Brolsma, N. van der Zwaluw, et al., Food sources of vitamin D and their association with 25hydroxyvitamin D status in Dutch older adults. The Journal of steroid biochemistry and molecular biology, 2016.

38. Lehmann, U., H.R. Gjessing, F. Hirche, et al., Efficacy of fish intake on vitamin D status: a meta-analysis of randomized controlled trials. The American journal of clinical nutrition, 2015. 102(4): p. 837-847.

39. Lemming, E.W., C. Nälsén, W. Becker, et al., Relative validation of the dietary intake of fatty acids among adults in the Swedish National Dietary Survey using plasma phospholipid fatty acid composition. Journal of nutritional science, 2015. 4: p. e25.

40. Aronsson, C.A., K. Vehik, J. Yang, et al., Use of dietary supplements in pregnant women in relation to sociodemographic factors - a report from The Environmental Determinants of Diabetes in the Young (TEDDY) study. Public Health Nutr., 2013. 16(8): p. 1390-402.

41. Pouchieu, C., R. Levy, C. Faure, et al., Socioeconomic, lifestyle and dietary factors associated with dietary supplement use during pregnancy. PLoS One, 2013. 8(8): p. e70733.

42. World Health Organization, WHO recommendations on antenatal care for a positive pregnancy experience. 2016, World Health Organization: Geneva.

43. Saadatian-Elahi, M., N. Slimani, V. Chajès, et al., Plasma phospholipid fatty acid profiles and their association with food intakes: results from a cross-sectional study within the European Prospective Investigation into Cancer and Nutrition. The American journal of clinical nutrition, 2009. 89(1): p. 331-346.

44. Fekete, K., T. Marosvölgyi, V. Jakobik, et al., Methods of assessment of $n-3$ long-chain polyunsaturated fatty acid status in humans: a systematic review. The American journal of clinical nutrition, 2009: p. ajcn. 272301. 
Dietary sources of folate, vitamin D, and $n-3$ fatty 



\section{Chapter 9}

General discussion 
The main aim of this thesis was to provide more insight in dietary intake and nutrient status before and during pregnancy and the association with development of gestational diabetes (GDM). In addition, we studied adverse pregnancy outcomes in women who received treatment after diagnosis of GDM and addressed two methodological topics important in nutrition research: assessment of diet quality, and bias in diet-disease associations due to measurement error.

The findings of these studies are summarized in Table 9.1. To be able to assess adherence to the Dutch dietary guidelines of 2015 the Dutch Healthy Diet 2015 (DHD15) index score was developed, which appeared to be a good marker of diet quality (chapter 2). Combining FFQ and 24-hour recall (24hR) dietary intake estimates was done using regression calibration and enhanced regression calibration and was found to reduce bias in diet-disease associations (chapter 3). Using data from medical file records, it was observed that women who were diagnosed with GDM and subsequently received treatment had higher risk of laceration but not of other adverse pregnancy outcomes than women with normal glucose tolerance during pregnancy; highest risks were observed for women with borderline gestational diabetes (BGDM) (chapter 4). Pre-pregnancy dietary intake was associated with GDM development in a large Australian population-based cohort study, with a low carbohydrate diet and higher cereal intake being associated with a higher risk of GDM (chapter 5), and with a higher fibre, fruit and fruit juice intake (chapter 5 ) and micronutrient adequacy (chapter 6 ) being associated with a lower risk of GDM. No clear associations between diet quality, micronutrient intake and micronutrient status levels, measured throughout pregnancy with fasting glucose, 2-hour glucose and HbA1c levels were observed in a small-scale study of Dutch women (chapter 7). In this study consisting predominantly of highly educated women, a high percentage of inadequate intake of folate, vitamin D and $n-3$ fatty acids eicosapentaenoic acid (EPA) and docosahexaenoic acid (DHA) was observed in the preconception period, as well as inadequate folate and vitamin D status (chapter 8).

In chapters 2-8, methodological considerations specific for the respective chapters have been addressed. The following paragraphs will discuss overarching methodological considerations and how the main findings of this thesis fit with the existing literature. First, a reflection is given on issues related to GDM diagnosis and dietary assessment, followed by a discussion regarding different aspects of the design of the studies used. Finally, suggestions for further research, implications for public health and clinical practice, and an overall conclusion will be presented. 


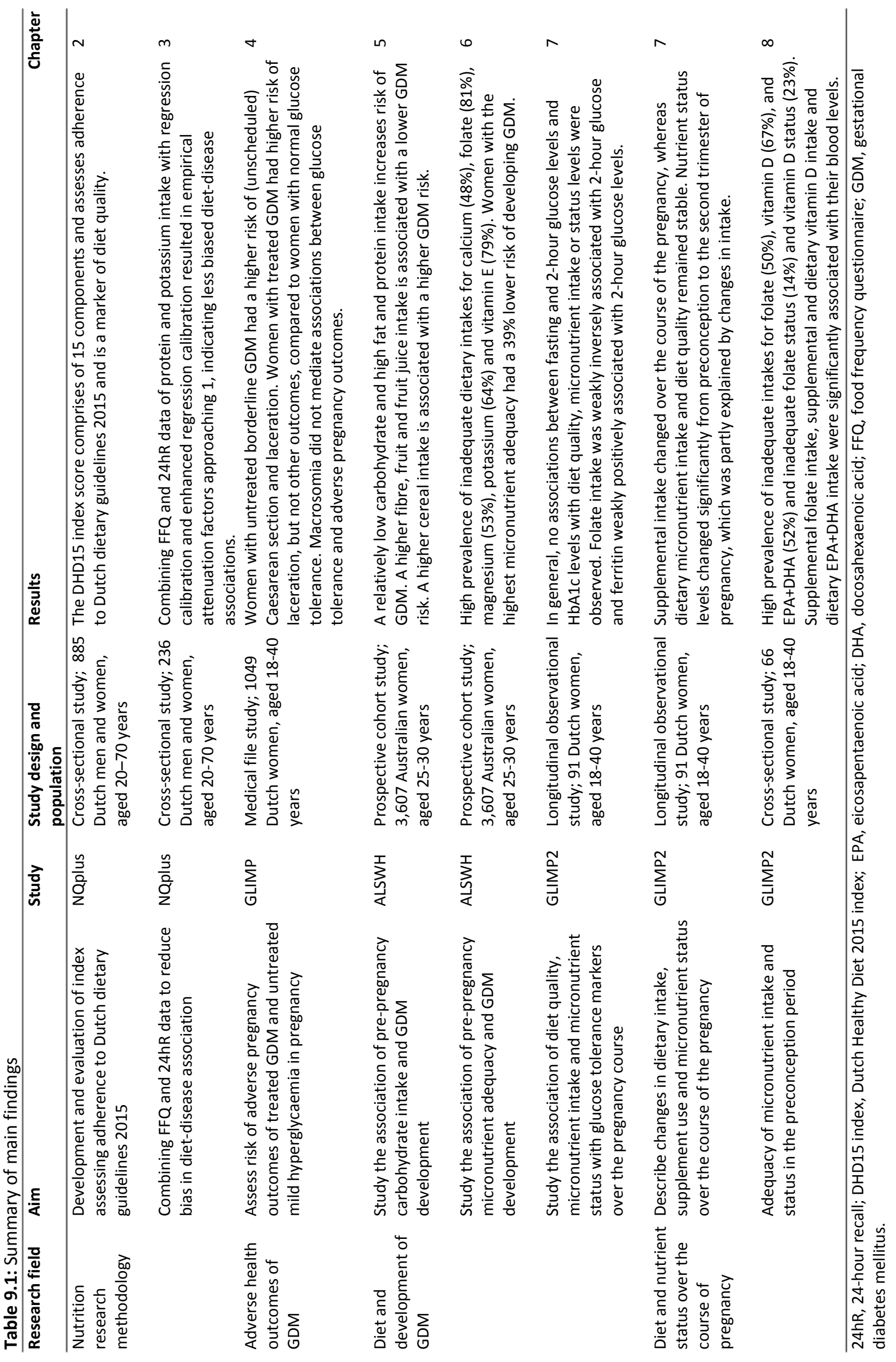




\section{GDM assessment}

One of the major challenges in GDM research is the lack of uniform diagnostic criteria. In this thesis data was used from different studies from two countries that used different criteria. In the GLIMP (chapter 4) and GLIMP2 study (chapter 7) results from the oral glucose tolerance test (OGTT) were available, making it possible to recalculate prevalences based on different sets of diagnostic criteria or work with continuous glucose values. In the ALSWH study (chapter 5, 6), however, only GDM diagnosis (yes/no) was reported, and thus based on one specific set of diagnostic criteria used in Australia at that time [1]. There has been a call for uniform diagnostic criteria [2-4]. In 2010, the International Association of Diabetes and Pregnancy Study Group (IADPSG) has proposed criteria based on the results of the landmark Hyperglycaemia and Adverse Pregnancy Outcome (HAPO) study [5]. These criteria have been adopted by several important institutions, including the World Health Organization (WHO) and American Diabetes Association, but not all, e.g. American College of Obstetricians and Gynaecologist [6]. Therefore, not every country has endorsed and implemented the new guidelines.

\section{GDM diagnostic criteria and adverse pregnancy outcomes}

An important aspect in the discussion on GDM diagnosis and its treatment is the increased risk of adverse pregnancy outcomes when GDM stays unrecognized. In chapter 4, risk of common adverse pregnancy outcomes including Caesarean section, assisted vaginal delivery, laceration, shoulder dystocia and post-partum haemorrhage were compared between three groups based on glucose tolerance status: women diagnosed with GDM (fasting glucose $\geq 6.1 \mathrm{mmol} / \mathrm{l}$ and/or 2-hour glucose $\geq 7.8$ $\mathrm{mmol} / \mathrm{l}$ after 75g OGTT) who received treatment, women with BGDM (1-hour glucose $\geq 7.8 \mathrm{mmol} / \mathrm{I}$ after $50 \mathrm{~g}$ glucose challenge test (GCT) and normal $75 \mathrm{~g}$ OGTT result) who did not receive treatment and women with normal glucose tolerance (normal 50g GCT result) during pregnancy. This study used medical file data from women who underwent GDM screening and delivered in hospital Gelderse Vallei Ede in 2010-2014. Hospital Gelderse Vallei Ede used a two-step universal screening strategy during the study period, which allowed us to define a group of women with BGDM that based on a one-step approach would have been included in the normal glucose tolerance group. Furthermore, as all women included in the study underwent GDM testing, regardless of whether they were at high risk, i.e. universal screening, all cases of GDM were identified. In this study the WHO criteria from 1999 [7] were used, which has the lowest 2-hour glucose cut-off value.

In our study, most women were diagnosed with GDM based on an abnormal 2-hour post-glucose load level. Few studies have investigated whether abnormal fasting glucose or abnormal 2-hour postglucose load levels are more indicative of adverse pregnancy outcomes [8, 9]. In the study of Koning et al. women with abnormal fasting glucose levels were at higher risk of gestational hypertension, 
induction of labour, planned Caesarean section, Apgar score $<7$ and admission to neonatology, whereas women with normal fasting glucose $(<5.1 \mathrm{mmol} / \mathrm{L})$ and abnormal 2-hour glucose level ( $\geq 7.8$ $\mathrm{mmol} / \mathrm{l})$ were not at increased risk compared to the normal glucose tolerance group [9]. However, these women with abnormal 2-hour glucose levels received treatment whereas the abnormal fasting glucose group did not. The study of Black et al., in which women received no treatment, observed that women with an elevated fasting but normal 2-hour glucose level had a higher risk of large for gestational age infants, but not for other adverse pregnancy outcomes compared to women with normal glucose tolerance [8]. In contrast, women in this study with abnormal 2-hour post-glucose level and normal fasting glucose had higher risk of preterm delivery, shoulder dystocia, gestational hypertension and hyperbilirubinemia compared to women with normal glucose tolerance [8]. These studies raise the question whether or not to keep the lower 2-hour glucose level of $7.8 \mathrm{mmol} / \mathrm{l}$ for diagnosis of GDM rather than the $8.5 \mathrm{mmol} / \mathrm{l}$ cut-off suggested in the 2010 International Association of Diabetes and Pregnancy Study Group (IADPSG) criteria [5], endorsed by the WHO and the American Diabetes Association (ADA) [10, 11].

The results of our study go even further and indicate that women with an abnormal 50 grams glucose challenge test (1-hour glucose $\geq 7.8 \mathrm{mmol} / \mathrm{l}$ ), but normal 75 grams OGTT (fasting glucose $<6.1 \mathrm{mmol} / \mathrm{I}$ and 2-hour glucose $<7.8 \mathrm{mmol} / \mathrm{l}$ ) had an increased risk of several common adverse pregnancy outcomes including macrosomia (i.e. birth weight $>4000$ g), laceration and (unscheduled) Caesarean section. However, a two-step universal screening approach is necessary to identify these women at risk. Main arguments of critics opposing to lower diagnostic criteria for GDM (i.e. implementation of IADPSG criteria) are the resulting steep increase in GDM cases and subsequent treatment costs [12, 13]. In addition, more cases of GDM will generate a greater workload for obstetrician, endocrinologist, and dietician services. For two hospital units in Australia it was estimated that overall workload would increase by $20-60 \%$, which might require a structural change in health care services [14].

\section{Dietary assessment}

\section{Complexity of diet}

Diet consists of different nutrients of which some exert beneficial health effects and others exert detrimental effects, and combinations present in foods and dietary patterns might have synergistic or antagonistic effects. In this thesis, several aspects of the diet, including dietary patterns (chapter 5, 7), foods (chapter 5 ) and micronutrients (chapter 6,7) in relation to GDM development have been studied. 


\section{Dietary patterns}

People do not consume single nutrients or foods, but combinations of foods. These combinations of foods may be interactive and synergistic and can be captured with dietary pattern analysis [15-18]. Dietary guidelines represent the current state of scientific knowledge regarding the effect of nutrients and foods on the development of chronic diseases [19]. In chapter 2, we developed and evaluated the Dutch Healthy Diet 2015 (DHD15) index score assessing adherence to the Dutch dietary guidelines 2015. The DHD15 index score proved to be a good measure of diet quality as it was associated with nutrient density. Furthermore, another study observed that higher adherence to the DHD15 index was associated with lower rates of mortality [20]. The DHD15 index is an a priori index score as it has predefined components and cut-off values. The advantage of a priori dietary patterns is that the predefined set of components and cut-off values can be applied in different study populations, which allows comparisons across different studies and populations [15]. However, the DHD15 index is designed for the Dutch population as it is based on the Dutch dietary guidelines 2015 [21]. Recommended intakes of these dietary guidelines were set taken the consumption pattern of the Dutch population into account, but might not be applicable to populations with on average higher or lower food group intakes. It is thus important to use diet scores appropriate to the study population.

In this thesis, two dietary pattern scores were used in relation to GDM development: the DHD15 index score used in chapter 7 and the low-carbohydrate diet (LCD) score used in chapter $\mathbf{5}$. The LCD score has been used in one other study investigating its association with GDM development [22]. The LCD score is a measure of macronutrient composition and both we (chapter 5) and Bao et al. observed a higher risk of GDM with a higher LCD score, reflecting a relative low carbohydrate intake and a relative high fat and protein intake [22]. We were the first to study the newly developed DHD15 index score in relation to glucose tolerance during pregnancy. We did not observe a significant association between the DHD15 index score and fasting glucose, 2-hour glucose and HbA1c levels during pregnancy in the total study population, but the DHD15-index was inversely associated with fasting glucose in a sensitivity analysis, in which participants with only one measurement were excluded (chapter 7). Other dietary patterns investigated in relation to GDM development are: Mediterranean Diet Score (MDS) [23-25], Healthy Eating Index (HEI) [25, 26], Dietary Approaches to Stop Hypertension (DASH) [23, 25], the Healthy Food Intake Index (HFII) which assesses adherence to the Nordic Nutrition Recommendations [27], and Western and prudent dietary patterns obtained with a posteriori dietary patterns $[26,28-33]$. Except for the LCD score and the Western dietary pattern score, the MDS, HEI, DASH, and HFII reflect a healthier diet with higher adherence; and higher adherence to any of these scores was associated with a significantly lower risk of GDM, except for the HFII. The Western dietary pattern was associated with a higher risk of GDM [43-47]. Although these dietary patterns have been 
derived in different ways, several food groups are included in the majority of these "healthier diet" patterns and are summarized in Table 9.2. Based on the results from these observational studies it is probable that a dietary pattern characterized by high intakes of fruit, vegetables, legumes, fish, nuts, whole grains and low intakes of red and processed meat, snacks, and added sugar lowers the risk of developing GDM.

Table 9.2. Components of dietary pattern scores reflecting a healthier diet that are associated with lower risk of gestational diabetes.

\begin{tabular}{|c|c|c|c|c|c|c|}
\hline & \multicolumn{5}{|c|}{ A priori } & \multirow{2}{*}{$\begin{array}{c}\text { A posteriori } \\
\text { Prudent }\end{array}$} \\
\hline & MDS & DASH & $\mathrm{HEI}$ & $\mathrm{HFII}$ & DHD15 & \\
\hline \multicolumn{7}{|l|}{ Higher intake is encouraged } \\
\hline Fruit & $x$ & $x$ & $\mathrm{x}$ & $x$ & $\mathrm{x}$ & $x$ \\
\hline Vegetables & $x$ & $x$ & $x$ & $x$ & $x$ & $x$ \\
\hline Nuts & $x$ & $x$ & $\mathrm{x}$ & & $x$ & $\mathrm{x}$ \\
\hline Legumes & $x$ & $x$ & $x$ & & $x$ & $x$ \\
\hline White: red meat ratio & & & $x$ & & & $x$ \\
\hline Fish and seafood & $x$ & & & $x$ & $x$ & $x$ \\
\hline Whole grains & $x$ & $x$ & & & $x$ & \\
\hline Whole grains: refined grains & & & & $x$ & $x$ & $x$ \\
\hline Cereal fibre & & & $x$ & & & \\
\hline Low-fat dairy & & $x$ & & $x$ & & $\mathrm{x}$ \\
\hline MUFA or PUFA:SFA & $x$ & & $x$ & $x$ & $x$ & \\
\hline \multicolumn{7}{|c|}{ Low/moderate intake is encouraged } \\
\hline Trans fat & & & $x$ & & & \\
\hline Red and processed meats & $x$ & $x$ & & & $x$ & \\
\hline Sweetened beverages & & $x$ & & $x$ & $x$ & $x$ \\
\hline Alcohol & $x$ & & $x$ & & $x$ & $x$ \\
\hline Sodium & & $x$ & & & $x$ & \\
\hline Added sugar & & & & $x$ & & \\
\hline
\end{tabular}

MDS, Mediterranean diet score; DASH, dietary approaches to stop hypertension; HEI, healthy eating index; HFII, healthy food intake index; DHD15, Dutch healthy diet index 2015; MUFA, mono-unsaturated fatty acids; PUFA, poly-unsaturated fatty acids; SFA, saturated fatty acids;

\section{Foods}

A better understanding of single foods and nutrients driving the association between diet and GDM as observed with dietary patterns can support dietary intervention development and nutritional counselling. Thus, analysis of individual foods and nutrients can complement dietary pattern analysis [15]. In chapter 5, the intake of several carbohydrate-rich food groups in relation to GDM development was investigated, and we observed that a higher intake of fruit and fruit juice was associated with a lower risk of GDM, whereas a higher cereal intake was associated with a higher risk of GDM. The number of studies investigating individual foods in relation to GDM development are limited (see Table 1.1 (chapter 1) for an overview of reported associations). Most statistically significant associations were reported in studies using the Nurses' Health Study II data; a positive association was observed 
for consumption of potatoes, red meat, processed meat, fried foods and sugar-sweetened cola [32, 34-36], whereas higher intake of nuts and cereal and fruit fibre were associated with lower risk of GDM [37-39]. The study of Karamanos using data of 1000 pregnant women from 10 Mediterranean countries reported a lower risk of GDM with higher intakes of potatoes, cereal and a higher risk with higher intakes of olive oil and cheese [24]. The discrepancy in results for single food items, for example, cereal intake, might be due to other products consumed with these specific foods, i.e. lack of context or differences in preparation methods. For example in the US, potatoes are more commonly consumed by women with a lower socioeconomic status who have in general a less healthy lifestyle and potatoes are often fried or baked [40], whereas in Mediterranean countries potatoes are considered a vegetable and higher intakes might be indicative of women with a higher overall vegetable intake and a more healthy lifestyle in general. For other foods, the nutrient content might be important to take into account. For example, cereal can contain high amounts of cereal fibre, but can also be a source of added sugar. However, up to date the evidence is too limited to make specific food recommendations for the prevention of GDM other than the dietary guidelines for the general population.

\section{Macronutrients}

The first studies investigating the relationship between diet and GDM paid most attention to macronutrient intake and composition. In general, total fat and saturated fat were associated with an increased risk of GDM, whereas results for polyunsaturated fat, protein and carbohydrates were mixed and thus inconclusive. Most conclusive evidence was observed in studies that took the correlation between and substitution of macronutrients into account, which is comparable to the LCD score used in chapter 5 . Our results and those of others $[22,41]$ indicate that a lower carbohydrate intake in combination with a higher fat and protein intake is associated with higher risk of GDM, and especially saturated fat and protein from animal origin were found to be detrimental [22, 37, 42].

\section{Fibre and glycaemic index (GI)}

Another dietary factor that has received ample attention in relation with GDM development is fibre, as epidemiological evidence relates high fibre and whole grain intake with a lower risk of type 2 diabetes [43]. Several, but not all, studies have observed a lower risk of GDM with a higher fibre consumption [39, 44-52]. In chapter 5, we also report a lower risk of GDM with higher fibre consumption, however adjustment for BMI attenuated the association. Dietary fibre can be divided into two groups based on water solubility. Whole grain products mainly contain insoluble fibre [53], which has been associated with intestinal transit time [54] and may modulate the composition and metabolism of gut microbiota, which can impact glucose homeostasis via several mechanisms [55]. On the other hand, fruit and vegetables contain more soluble fibres [53]. Soluble fibres can create a gellike substance in the stomach, which can delay gastric emptying and thus slow glucose absorption [56- 
58]. In our study (chapter 5), we observed a lower risk of GDM with higher fruit consumption, but not cereal, whereas in the study of Zhang et al. both fruit and cereal fibre, but not vegetable fibre, were associated with lower GDM risk [39]. Therefore, it might be important to take the type of fibre into account. Furthermore, a high fibre diet often coincides with a low GI diet [59]. There is substantial evidence that low GI diets may reduce insulin resistance and hyperlipidaemia [60,61]. However, only few studies have been done on a low Gl diet in pregnancy. Results of these studies suggest that pregnant women could benefit from low GI diets to lower maternal glycosylated haemoglobin, plasma glucose, birth weight, and reduce insulin requirements of women with GDM [62-64].

\section{Micronutrients}

Micronutrients are involved in a vast array of physiological processes such as enzyme activity, signal transduction and transcription pathways, biological functions and oxidative stress [65]. Recent reviews have shown that there is a relation between diet and the development of glucose intolerance $[56,66$, 67]; however, data regarding the impact of specific micronutrients on glucose homeostasis and GDM are just beginning to emerge [56]. Vitamin A, D, K, C and E, calcium, magnesium, zinc, chromium, and sodium are suggested to have a positive effect on glucose homeostasis, but underlying mechanisms are not yet established, or need further confirmation $[66,67]$. Higher intakes of iron, on the other hand, are suggested to have a detrimental effect on glucose homeostasis, as excess iron can induce oxidative stress that can damage beta cell function $[68,69]$.

In chapter 6, we investigated prevalence of micronutrient deficiency and association of micronutrient adequacy and risk of GDM development. We did not observe an association between a single nutrient deficiency and risk of GDM, but overall micronutrient adequacy, i.e. meeting intake recommendations for 13 micronutrients, was associated with a lower risk of GDM. This might suggest that although individual micronutrients have a distinct role in glucose homeostasis, the effect of one single micronutrient on glucose homeostasis and consequently GDM development might be too small to be detected. A limitation of the ALSWH study used in chapter 6, was the absence of nutrient status markers. Nutrient status markers could provide more information on underlying physiology and mechanisms, as intake does not necessarily reflect the amount of nutrient available to the body [70]. Nutrient status is the link between intake and health effects. In the GLIMP2 study, chapter $\mathbf{7}$ and 8, both intake and status markers of folate, vitamin B6, B12, D, and iron were available. We observed no significant associations of micronutrient intake and status with markers of glucose tolerance (chapter 7), except for a weakly inverse association between folate intake and 2-hour glucose levels and a weakly positive association between iron status and 2-hour glucose levels. Thus, no clear associations could be drawn regarding the role of micronutrients in relation to GDM development. A limitation of the GLIMP2 study is that we investigated only five micronutrients and the study might have been 
underpowered. The few studies that included both intake and status [71-73], in general, observed stronger associations between nutrient status and GDM risk than between nutrient intake and GDM risk, advocating the inclusion of nutrient status markers in future studies investigating micronutrients and GDM risk.

In chapter 7, we also described changes in nutrient status during pregnancy. We observed that all status markers significantly changed during pregnancy, and that this was not always related to intake. Furthermore, status markers were more often associated with supplement intake than dietary intake. Thus, both gestational age and supplement use are important to take into account when status markers during pregnancy are used, for example to determine prevalence of deficiency. Although diet is linked to development of GDM, the role of micronutrients remains unclear and more research including both intake and status markers is needed to unravel the potential mechanisms by which diet can affect GDM development and the specific (micro)nutrients involved.

\section{Measurement error}

Dietary intake assessment is prone to measurement error, which can lead to biased diet-disease associations and prevalence estimates. In chapter 3, we observed that combining dietary intake data from $24 \mathrm{hR}$ and $F F Q$ with regression calibration $(\mathrm{RC})$ and enhanced regression calibration (ERC) reduced bias in diet-disease associations. An advantage of these methods is that the statistical method is quite simple and most nutrition researchers should be able to apply them, in contrast to more advanced approaches such as the $\mathrm{NCl}$-method $[74,75]$, which requires advanced statistical computations. Disadvantages of regression calibration have a more practical nature. The development of web-based $24 \mathrm{hR}$ have reduced costs and burden for the researcher [76], but still requires additional time from participants. Furthermore, multiple dietary assessments might reduce willingness to participate or increase dropout, which could affect representativeness of the study population. This is especially of concern, when participants with a low educational level or low socioeconomic status, which are already underrepresented in most nutrition research studies, are the most affected. However, obtaining unbiased diet-disease associations is of the utmost importance as well. The development of new technological dietary assessment tools using smartphone apps might provide part of the solution [82].

In chapter 7, we were able to use ERC to estimate micronutrient intake estimates as we had dietary estimates from both FFQ and two $24 \mathrm{hR}$ at each measurement moment. However, in chapter 8, we did not use ERC, although we had both the FFQ and two $24 \mathrm{hR}$ available. The reason was that the sample size for this study was limited to 66 women. In the ALSWH study, used in chapter $\mathbf{5}$ and $\mathbf{6}$, dietary intake was assessed with only a FFQ, thus ERC and RC were not possible. Although the FFQ was 
validated for a wide range of macro- and micronutrients (correlation coefficients ranging from 0.28 for vitamin A to 0.78 for carbohydrates) [77], bias due to measurement error is likely to have occurred, and affected observed diet-disease associations to some extent [78].

\section{Lifestyle clustering}

The main focus of this thesis is the association of diet with GDM. However, diet is just one part of a larger set of lifestyle behaviours. Other lifestyle factors include physical activity, smoking, alcohol intake and sleep. These behaviours tend to cluster, e.g. people with unhealthy dietary habits, are more often physically inactive, smoke more often and are more likely to consume more alcohol, and viceversa [79-81]. Two recent studies have investigated the association between a healthy lifestyle, including a healthy diet, and development of GDM. They observed that adherence to a healthy lifestyle was associated with a significant $75-80 \%$ decrease in risk of developing GDM compared to women with an unhealthy lifestyle $[82,83]$, but only few women were in the extremes, i.e. adhering to all healthy or all unhealthy lifestyle behaviours. These studies demonstrate the importance of taking other lifestyle factors into account when investigating diet in relation to GDM development. If other lifestyle factors are not accounted for, the observed effect might be due to these unmeasured factors instead of dietary intake, i.e. residual confounding. In both the ALSWH and GLIMP2 studies several other lifestyle factors, including physical activity, smoking and alcohol intake were assessed and results presented in chapter 5-8 are adjusted for these factors. However, inaccurate measurements, e.g. physical activity, and unmeasured factors, e.g. sleep, might still have led to residual confounding.

\section{Study design}

There are several study design aspects that need to be discussed in order to be able to place the emerging body of evidence regarding the association of diet with the development of GDM, including results presented in this thesis (chapters 5-7) into context. These include study population, time frame, effect size and type of study (i.e. observational vs. intervention) and will be discussed below.

\section{Study population}

In this thesis, two different study populations have been used to investigate the role of diet in the development of GDM. In chapters 5 and $\mathbf{6}$ data from the ALSWH study, a large population-based prospective cohort study was used. The advantage of a population-based cohort study is the high external validity. However, as prevalence of GDM is around 5\% in Caucasian populations [2], the number of cases is still rather low. An alternative to ensure enough cases and thus enough statistical power is studying high-risk populations with GDM prevalence up to $50 \%[84,85]$. This was done in the 
GLIMP2 study, which oversampled women with a history of GDM, a previous macrosomic infant, polycystic ovarian syndrome (PCOS), and overweight. Oversampling of women at high risk of GDM in the GLIMP2 study resulted in a GDM incidence of $10 \%$. A consequence of selecting a high-risk study population is that the degree of gradually developed, underlying, subclinical insulin resistance in the years before pregnancy (see figure 9.1) might be too severe for dietary factors to have any beneficial effect $[51,86,87]$, thus potentially limiting the ability to detect an effect of diet. Furthermore, BMI can also act as a mediator in the association between diet and GDM [88], and should be considered when selecting only obese women, as is done in some other studies [89-93]. In addition to having a higher risk of GDM, the GLIMP2 study population consisted of women who were mostly highly educated and with an interest in health, and thus more likely to be more health conscious than the general population. This may not only have reduced external generalizability, but potentially may also have limited variation in dietary intake and thus the ability to detect an association with GDM.

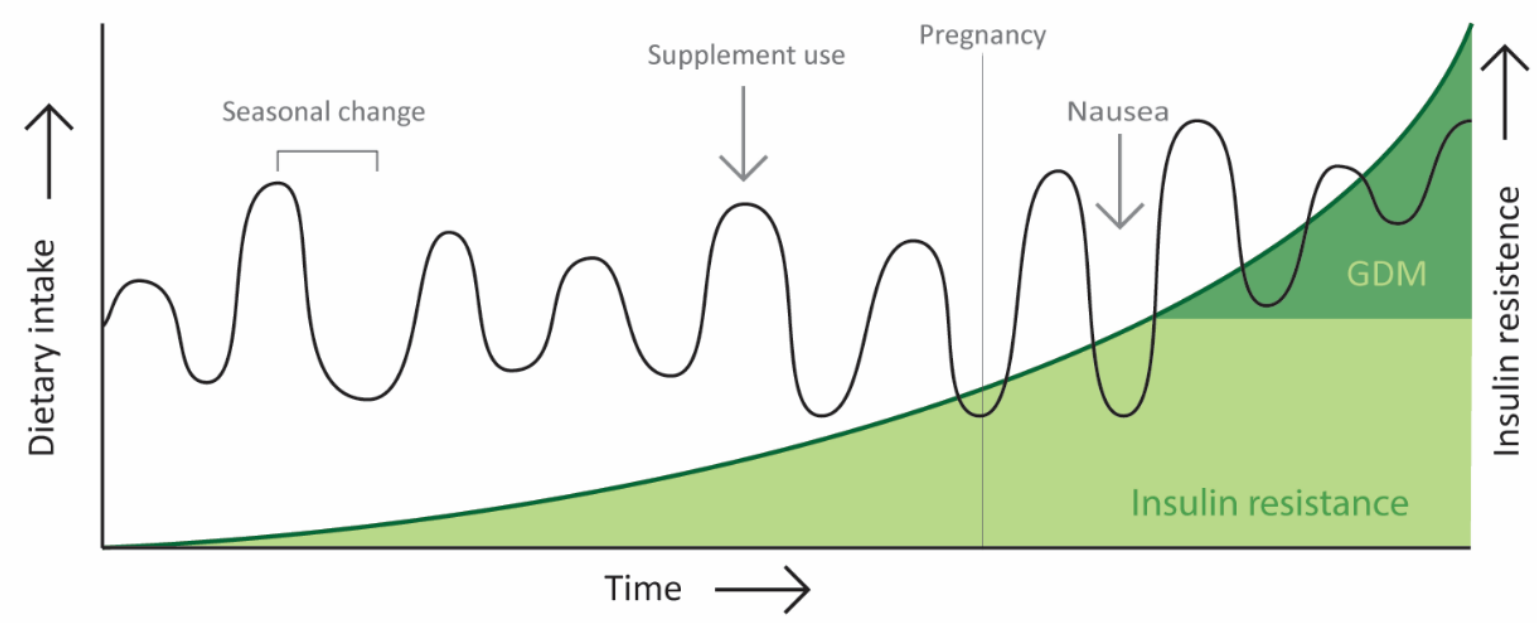

Figure 9.1: A schematic overview of how dietary intake might vary over time and can be influenced by specific events in relation to insulin resistance/GDM development.

\section{Time frame}

Diet is a lifelong exposure and it is important to take the timing of dietary assessment into account when studying diet-disease associations, especially since chronic disease development does not happen overnight, but develops over time. To avoid reverse causation, dietary assessment should be done before disease diagnosis, in this case before GDM diagnosis, as is done in the ALSWH and GLIMP2 studies (chapters 5-7). Furthermore, depending on the method used, dietary intake may reflect intake at a certain point in time, which is not necessarily representative of habitual intake. For example, seasonal variation might influence dietary intake. Pregnancy is a major life event that can influence 
dietary intake. For example, food safety recommendations are given to ensure avoidance of contaminants, but also pregnancy-induced food aversions, nausea and vomiting can affect dietary intake. Therefore, diet measured several years before pregnancy (e.g. ALSWH) may not reflect the diet consumed during pregnancy, and vice versa, diet assessed during pregnancy might not reflect habitual pre-pregnancy diet, whereas both can have an effect on the development of insulin resistance (see figure 9.1). In the ALSWH study, two FFQs were administered six years apart and sensitivity analyses in which data from both were combined as long-term dietary intake showed similar associations as those based on the FFQ closest to the pregnancy (chapter 5, 6). In theory, multiple measurements both in the preconception and pregnancy period are necessary to accurately capture dietary intake and changes over time. In chapter 7, we assessed diet quality and micronutrient intake in the preconception period, on average 13 weeks before conception, and twice during pregnancy. We observed that dietary intake remained stable over this time frame. Combined with results obtained from others [94-96], it is likely that, although total intake and intake of specific foods may change in the year before the pregnancy, the effect on diet quality and micronutrient intake is limited. Furthermore, in the GLIMP2 study, we did not observe a significant effect of nausea and vomiting on dietary intake estimates.

\section{Observational versus intervention studies}

Results presented in this thesis with respect to the association between diet and GDM development (chapters 5-7) are based on observational data, as are the majority of results of other studies. However, randomized controlled trials (RCTs) are needed to prove causality. A recent systematic review and meta-analysis summarized results from 23 RCTs investigating combined diet and exercise interventions to prevent GDM compared to no intervention and concluded that there was a possible reduced risk of GDM (average risk ratio $0.85,95 \%$ confidence interval 0.71 to $1.01 ; 6633$ women; 19 RCTs), but that the quality of the evidence was moderate [97]. Furthermore, there was large heterogeneity observed in the type of dietary intervention given, so effective dietary components are difficult to disentangle.

Another limitation of RCTs is that they often start during pregnancy, due to feasibility reasons, and thus have only a short time window (e.g. from 12 weeks of gestation at inclusion to 24-28 weeks of gestation for GDM testing) to change dietary intake and for this change in diet to have an effect on insulin resistance and glucose homeostasis. Therefore, new trials aim to start already in the preconception period. One of these trials is the recently started large multicentre, multi-ethnic randomized "Nutritional intervention Preconception and during Pregnancy to maintain healthy glucose metabolism and offspring health (NiPPeR)" trial investigating the effect of an optimized nutritional drink with myo-inositol, micronutrients and probiotics on glucose tolerance that starts with 
the intervention in the preconception phase [98]. A major challenge of this study, however, is the large number of women needed, $n=1800$, to be able to study the aimed 600 pregnancies, as not all women will conceive and anticipating dropout.

\section{Diet during pregnancy in a broader perspective}

A high prevalence of inadequate pre-pregnancy dietary intake of folate, calcium, magnesium, potassium and vitamin E was observed in an Australian population (chapter 6) and a large proportion of Dutch women who wished to become pregnant did not meet recommendations for folate, vitamin D and $n-3$ fatty acids intake (chapter 8). These suboptimal prepregnancy intakes were also observed in other populations [99-102], and although more prevalent among lower educated women [102, 103], our results indicate that even in highly educated women recommendations are not met. In chapter 7 , we observed that diet quality, not considering supplement use, did not change in women from preconception to the end of the second trimester. This is in line with other studies observing that, although energy intake increased, diet quality and micronutrient intake did not change $[101,104,105]$. This is of concern, as pregnancy is a potential window of opportunity to improve diet quality [106-108]. More and more focus has been placed on starting interventions already in the preconception period, when maternal diet and nutrient status can be improved to provide an optimal intrauterine environment before foetal development starts. However, preconception care is not a standardized part of general health care [109] and awareness of preconception health among women and health professionals is low, and responsibility for providing preconception care is unclear [106].

Improving diet quality during pregnancy and ideally in the preconception period may not only lead to a lower risk of developing GDM. By providing an optimal intrauterine environment, chronic disease risk in childhood and adulthood may be reduced as postulated by the developmental origins of health and disease $(\mathrm{DOHaD})$ hypothesis $[110,111]$. In addition, improving diet quality in the preconception period might reduce subfertility rates [112-114], gestational weigh gain [115], gestational hypertension [116], preeclampsia [117], intrauterine growth retardation [118], preterm delivery [119], risk of Caesarean section [115] and potentially many others [120].

As demonstrated in chapters $\mathbf{7}$ and $\mathbf{8}$, supplementation can help in meeting micronutrient intake recommendations and improving micronutrient status. However, some considerations should be taken into account regarding supplement use. Women who take prenatal supplements are often women with a higher educational level, who, in general, already achieve higher intakes of micronutrients [121, 122]. Health policies encouraging supplement use might not reach the women 
with the lowest intake levels, but increase supplement consumption in health-conscious women with partly already adequate intake levels, who are consequently at risk of excess intake above the upper level of intake [101]. This might especially occur in countries with mandatory fortification of bread, for example, with folate, such as in the United States, United Kingdom and Australia. In the GLIMP2 study (chapter 7) $14 \%$ of the women consumed folate above the upper level of intake. For other micronutrients, we observed no intake above upper levels. Intake of micronutrients above the recommended upper level can cause medical complaints such as nerve damage with long-term excessive intake of vitamin B6 [123, 124]. Furthermore, little is known about whether high doses of micronutrients might affect development of the unborn child, but in light of the DOHaD hypothesis, this seems plausible. A recent study has found a link between high doses of folic acid during pregnancy (>5 mg/d) and impaired psychomotor development at 12-23 months of age [125]. More research is needed to unravel the adverse effects of excess micronutrient intake, especially for potential adverse effects in the offspring.

Thus, adequate dietary intake is important and a supplement can help in achieving recommended intakes, but caution is warranted with respect to the doses and should not exceed the recommended daily allowance.

\section{Recommendations for future research}

Current scientific evidence suggests a beneficial role of a healthy diet in GDM prevention, especially when a healthy diet is achieved before pregnancy. However, more research is needed to further understand the role of diet in the development of GDM and defining the foods and nutrients driving observed associations, as well as potential underlying mechanisms. This information can be used for tailoring dietary interventions to prevent GDM.

As outlined above, current intervention studies may be limited by a short time frame for dietary changes to exert an effect on glucose homeostasis and insulin resistance. Starting in the preconception period is thus a logical next step. Recruitment of women in the preconception period was the biggest challenge in our small-scale GLIMP2 study. The currently ongoing NiPPeR trial [98] will not only provide information on the effect of a nutritional drink on GDM development, but also provide vital information on feasibility and practicality of research in the preconception period on a large scale.

Furthermore, research should include both intake and status markers, as these complement each other and together they provide a more complete picture than either one alone. Our observations of changes in micronutrient intake, supplement use and status markers warrant additional research in a 
larger study sample. Multiple measurements throughout the pregnancy are preferable, especially regarding status markers. As supplement use can significantly influence status markers, detailed assessment of supplemental intake is desirable.

Prevention of GDM is preferred above treatment, but identification of women with GDM as well as timely treatment is essential. Although the IADSPG guidelines are endorsed by several leading organizations such as the WHO and ADA, the debate regarding screening and diagnostic criteria continues, especially in light of increasing prevalence and health care burden. It is important that future research will identify those at risk of adverse pregnancy outcomes and those who might benefit from treatment. Cost-effectiveness studies will give more insight in treatment and health care costs versus benefits from preventing adverse pregnancy outcomes.

Finally, efforts to optimize and improve dietary assessment methods to minimize bias should not be forgotten, as dietary intake estimates are the key stone in nutrition research.

\section{Implications for clinical and public health practice}

In the Netherlands, GDM screening is recommended [126], but there are no strict recommendations with regard to screening and diagnostic criteria. Results from this thesis underline the importance of timely screening and start of treatment, as women with (borderline) GDM have an increased risk of common adverse pregnancy outcomes. Furthermore, risk of GDM can potentially be reduced, by providing women with dietary counselling to improve diet quality. The discussion of potential food hazards is a common routine at the first prenatal visit, and dietary counselling could be amended to this. This requires proper informing and training of obstetricians, GPs, midwives and others involved. Dietary counselling at the first prenatal visit might not only reduce risk of GDM development, but could beneficially impact a number of pregnancy outcomes and potentially long term health of the foetus.

Ideally, dietary counselling is already provided in the preconception stage, to ensure an adequate nutrient status upon conception. Although the Health Council of the Netherlands already called upon the provision of proper and adequate preconception care a decade ago [127], results of this thesis indicate that more action is needed to improve intake and status of essential micronutrients in the preconception period. This might be partly due to the fact that it is unclear who is responsible for offering preconception care and that women are not aware of the possibility of preconception advice from health care providers [106]. A first step for reaching women in the preconception stage could be fertility clinics. As obesity is associated with reduced fertility [128], this might provide a high-risk group that could benefit from dietary counselling. 
Dietary counselling should include information regarding the importance of meeting dietary recommendations, how to improve diet quality, the potential benefit of supplement use, but also information regarding the correct dose and use of supplements and risk of excessive intake.

\section{Conclusions}

Based on the results described in this thesis as well as existing literature, it is probable that a healthy diet can reduce risk of developing gestational diabetes. Most convincing evidence is observed for diet in the pre-pregnancy period. In both an Australian and a Dutch population, diet in the pre-pregnancy period was considered suboptimal and intake of several vital micronutrients, including folate, was below recommended intakes for a large proportion of women. The pre-pregnancy period thus is an ideal situation for improving dietary intakes. Supplements can help bridging the gap between dietary intake and recommended intake, but caution regarding excessive intake is warranted. Furthermore, borderline gestational diabetes was associated with higher risk of common adverse pregnancy outcomes, stressing the urgency of gestational diabetes diagnosis and timely treatment. 


\section{References}

1. Hoffman, L., C. Nolan, J.D. Wilson, et al., Gestational diabetes mellitus--management guidelines. The Australasian Diabetes in Pregnancy Society. Med. J. Aust., 1998. 169(2): p. 93-7.

2. Buckley, B.S., J. Harreiter, P. Damm, et al., Gestational diabetes mellitus in Europe: prevalence, current screening practice and barriers to screening. A review. Diabet. Med., 2012. 29(7): p. 844-54.

3. Koning, S.H., K. Hoogenberg, H.L. Lutgers, et al., Gestational Diabetes Mellitus:current knowledge and unmet needs. J. Diabetes, 2016. 8(6): p. 770-781.

4. $\quad$ Ryan, E.A., Diagnosing gestational diabetes. Diabetologia, 2011. 54(3): p. 480-6.

5. International Association of Diabetes Pregnancy Study Groups Consensus Panel, International association of diabetes and pregnancy study groups recommendations on the diagnosis and classification of hyperglycemia in pregnancy. Diabetes Care, 2010. 33(3): p. 676-82.

6. Ryan, E.A., Clinical diagnosis of gestational diabetes. Clin. Obstet. Gynecol., 2013. 56(4): p. 774-87.

7. World Health Organization, Definition, diagnosis and classification of diabetes mellitus and its complications. 1999, World Health Organization: Geneva, Switzerland.

8. Black, M.H., D.A. Sacks, A.H. Xiang, et al., Clinical outcomes of pregnancies complicated by mild gestational diabetes mellitus differ by combinations of abnormal oral glucose tolerance test values. Diabetes Care, 2010. 33(12): p. 252430.

9. Koning, S.H., J.J. van Zanden, K. Hoogenberg, et al., New diagnostic criteria for gestational diabetes mellitus and their impact on the number of diagnoses and pregnancy outcomes. Diabetologia, 2017.

10. American Diabetes Association, Diagnosis and classification of diabetes mellitus. Diabetes Care, 2014. 37 Suppl 1: p. S81-90.

11. World Health Organization, Diagnostic criteria and classification of hyperglycaemia first detected in pregnancy. 2013, Geneva, Switzerland: World Health Organization.

12. Cundy, T., E. Ackermann, and E.A. Ryan, Gestational diabetes: new criteria may triple the prevalence but effect on outcomes is unclear. BMJ, 2014. 348: p. g1567.

13. Visser, G.H. and H.W. de Valk, Is the evidence strong enough to change the diagnostic criteria for gestational diabetes now? Am. J. Obstet. Gynecol., 2013. 208(4): p. 260-4.

14. Flack, J.R., G.P. Ross, S. Ho, et al., Recommended changes to diagnostic criteria for gestational diabetes: impact on workload. Aust. N. Z. J. Obstet. Gynaecol., 2010. 50(5): p. 439-43.

15. Hu, F.B., Dietary pattern analysis: a new direction in nutritional epidemiology. Curr. Opin. Lipidol., 2002. 13(1): p. 39.

16. Kant, A.K., Indexes of overall diet quality: a review. J. Am. Diet. Assoc., 1996. 96(8): p. 785-91.

17. Waijers, P.M., E.J. Feskens, and M.C. Ocke, A critical review of predefined diet quality scores. Br. J. Nutr., 2007. 97(2): p. 219-31.

18. van de Rest, O., A.A. Berendsen, A. Haveman-Nies, et al., Dietary patterns, cognitive decline, and dementia: a systematic review. Adv. Nutr., 2015. 6(2): p. 154-68.

19. Health Council of the Netherlands, Dutch dietary guidelines 2015. Publication no. 2015/24. 2015, Health Council of the Netherlands: The Hague.

20. Biesbroek, S., W.M.M. Verschuren, J.M.A. Boer, et al., Does a better adherence to dietary guidelines reduce mortality risk and environmental impact in the Dutch sub-cohort of the European Prospective Investigation into Cancer and Nutrition? Br. J. Nutr., 2017. 118(1): p. 69-80.

21. Health Council of the Netherlands, Dutch dietary guidelines 2015 - Background document dairy. Publication no. A15/32. 2015, Health Council of the Netherlands: The Hague.

22. Bao, W., K. Bowers, D.K. Tobias, et al., Prepregnancy low-carbohydrate dietary pattern and risk of gestational diabetes mellitus: a prospective cohort study. Am. J. Clin. Nutr., 2014.

23. Izadi, V., H. Tehrani, F. Haghighatdoost, et al., Adherence to the DASH and Mediterranean diets is associated with decreased risk for gestational diabetes mellitus. Nutrition, 2016. 32(10): p. 1092-6.

24. Karamanos, B., A. Thanopoulou, E. Anastasiou, et al., Relation of the Mediterranean diet with the incidence of gestational diabetes. Eur. J. Clin. Nutr., 2014. 68(1): p. 8-13.

25. Tobias, D.K., C. Zhang, J. Chavarro, et al., Prepregnancy adherence to dietary patterns and lower risk of gestational diabetes mellitus. Am. J. Clin. Nutr., 2012. 96(2): p. 289-95.

26. Tryggvadottir, E.A., H. Medek, B.E. Birgisdottir, et al., Association between healthy maternal dietary pattern and risk for gestational diabetes mellitus. Eur. J. Clin. Nutr., 2016. 70(2): p. 237-42.

27. Meinila, J., A. Valkama, S.B. Koivusalo, et al., Association between diet quality measured by the Healthy Food Intake Index and later risk of gestational diabetes-a secondary analysis of the RADIEL trial. Eur. J. Clin. Nutr., 2017. 71(4): p. 555-557.

28. Donazar-Ezcurra, M., C. Lopez-Del Burgo, M.A. Martinez-Gonzalez, et al., Pre-pregnancy adherences to empirically derived dietary patterns and gestational diabetes risk in a Mediterranean cohort: the Seguimiento Universidad de Navarra (SUN) project. Br. J. Nutr., 2017. 118(9): p. 715-721.

29. He, J.R., M.Y. Yuan, N.N. Chen, et al., Maternal dietary patterns and gestational diabetes mellitus: a large prospective cohort study in China. Br. J. Nutr., 2015. 113(8): p. 1292-300. 
30. Schoenaker, D.A., S.S. Soedamah-Muthu, L.K. Callaway, et al., Pre-pregnancy dietary patterns and risk of gestational diabetes mellitus: results from an Australian population-based prospective cohort study. Diabetologia, 2015. 58(12): p. 2726-35.

31. Shin, D., K.W. Lee, and W.O. Song, Dietary Patterns during Pregnancy Are Associated with Risk of Gestational Diabetes Mellitus. Nutrients, 2015. 7(11): p. 9369-82.

32. Zhang, C., M.B. Schulze, C.G. Solomon, et al., A prospective study of dietary patterns, meat intake and the risk of gestational diabetes mellitus. Diabetologia, 2006. 49(11): p. 2604-13.

33. Practicumhandleiding: Bepaling van de vetzuursamenstelling van cholesterolesters en fosfolipiden in plasma 1.0 Laboratorium Human Voeding, Wageningen UR. n.d.].

34. Bao, W., D.K. Tobias, F.B. Hu, et al., Pre-pregnancy potato consumption and risk of gestational diabetes mellitus: prospective cohort study. BMJ, 2016. 352: p. h6898.

35. Bao, W., D.K. Tobias, S.F. Olsen, et al., Pre-pregnancy fried food consumption and the risk of gestational diabetes mellitus: a prospective cohort study. Diabetologia, 2014.

36. Chen, L., F.B. Hu, E. Yeung, et al., Prospective study of pre-gravid sugar-sweetened beverage consumption and the risk of gestational diabetes mellitus. Diabetes Care, 2009. 32(12): p. 2236-41.

37. Bao, W., K. Bowers, D.K. Tobias, et al., Prepregnancy dietary protein intake, major dietary protein sources, and the risk of gestational diabetes mellitus: a prospective cohort study. Diabetes Care, 2013. 36(7): p. 2001-8.

38. Chen, L., F.B. Hu, E. Yeung, et al., Prepregnancy consumption of fruits and fruit juices and the risk of gestational diabetes mellitus: a prospective cohort study. Diabetes Care, 2012. 35(5): p. 1079-82.

39. Zhang, C., S. Liu, C.G. Solomon, et al., Dietary fiber intake, dietary glycemic load, and the risk for gestational diabetes mellitus. Diabetes Care, 2006. 29(10): p. 2223-30.

40. Mullie $\mathrm{P}$, Boniol M, and Autier P, Re: Pre-pregnancy potato consumption and risk of gestational diabetes mellitus: prospective cohort study [letter to the editor]. BMJ 2016; 352: h6898, 2016.

41. Saldana, T.M., A.M. Siega-Riz, and L.S. Adair, Effect of macronutrient intake on the development of glucose intolerance during pregnancy. Am. J. Clin. Nutr., 2004. 79(3): p. 479-86.

42. Bowers, K., E. Yeung, M.A. Williams, et al., A prospective study of prepregnancy dietary iron intake and risk for gestational diabetes mellitus. Diabetes Care, 2011. 34(7): p. 1557-63.

43. Aune, D., T. Norat, P. Romundstad, et al., Whole grain and refined grain consumption and the risk of type 2 diabetes: a systematic review and dose-response meta-analysis of cohort studies. Eur. J. Epidemiol., 2013. 28(11): p. 845-58.

44. Bennett, W.L., S.H. Liu, H.C. Yeh, et al., Changes in weight and health behaviors after pregnancies complicated by gestational diabetes mellitus: the CARDIA study. Obesity (Silver Spring), 2013. 21(6): p. 1269-75.

45. Bo, S., G. Menato, A. Lezo, et al., Dietary fat and gestational hyperglycaemia. Diabetologia, 2001. 44(8): p. 972-8.

46. Gonzalez-Clemente, J.M., O. Carro, I. Gallach, et al., Increased cholesterol intake in women with gestational diabetes mellitus. Diabetes Metab., 2007. 33(1): p. 25-9.

47. Helin, A., T.I. Kinnunen, J. Raitanen, et al., Iron intake, haemoglobin and risk of gestational diabetes: a prospective cohort study. BMJ open, 2012. 2(5).

48. Ley, S.H., A.J. Hanley, R. Retnakaran, et al., Effect of macronutrient intake during the second trimester on glucose metabolism later in pregnancy. Am. J. Clin. Nutr., 2011. 94(5): p. 1232-40.

49. Park, S., M.Y. Kim, S.H. Baik, et al., Gestational diabetes is associated with high energy and saturated fat intakes and with low plasma visfatin and adiponectin levels independent of prepregnancy BMI. Eur. J. Clin. Nutr., 2013. 67(2): p. 196-201.

50. Qiu, C., I.O. Frederick, C. Zhang, et al., Risk of gestational diabetes mellitus in relation to maternal egg and cholesterol intake. Am. J. Epidemiol., 2011. 173(6): p. 649-58.

51. Radesky, J.S., E. Oken, S.L. Rifas-Shiman, et al., Diet during early pregnancy and development of gestational diabetes. Paediatr. Perinat. Epidemiol., 2008. 22(1): p. 47-59.

52. Wang, Y., L.H. Storlien, A.B. Jenkins, et al., Dietary variables and glucose tolerance in pregnancy. Diabetes Care, 2000. 23(4): p. 460-4.

53. Lairon, D., S. Bertrais, S. Vincent, et al., Dietary fibre intake and clinical indices in the French Supplementation en Vitamines et Mineraux AntioXydants (SU.VI.MAX) adult cohort. Proc. Nutr. Soc., 2003. 62(1): p. 11-5.

54. Roehrig, K.L., The physiological effects of dietary fiber-a review. Food Hydrocolloid, 1988. 2(1): p. 1-18.

55. Davison, K.M. and N.J. Temple, Cereal fiber, fruit fiber, and type 2 diabetes: Explaining the paradox. J. Diabetes Complications, 2018. 32(2): p. 240-245.

56. Russell, W.R., A. Baka, I. Bjorck, et al., Impact of Diet Composition on Blood Glucose Regulation. Crit. Rev. Food Sci. Nutr., 2016. 56(4): p. 541-90.

57. Mclntosh, M. and C. Miller, A diet containing food rich in soluble and insoluble fiber improves glycemic control and reduces hyperlipidemia among patients with type 2 diabetes mellitus. Nutr. Rev., 2001. 59(2): p. 52-5.

58. Poutanen, K.S., P. Dussort, A. Erkner, et al., A review of the characteristics of dietary fibers relevant to appetite and energy intake outcomes in human intervention trials. Am. J. Clin. Nutr., 2017. 106(3): p. 747-754.

59. Bjorck, I. and H.L. Elmstahl, The glycaemic index: importance of dietary fibre and other food properties. Proc. Nutr. Soc., 2003. 62(1): p. 201-6.

60. Dickinson, S. and J. Brand-Miller, Glycemic index, postprandial glycemia and cardiovascular disease. Curr. Opin. Lipidol., 2005. 16(1): p. 69-75. 
61. Wolever, T.M., Carbohydrate and the regulation of blood glucose and metabolism. Nutr. Rev., 2003. 61(5 Pt 2): p. S40-8.

62. Louie, J.C., J.C. Brand-Miller, and R.G. Moses, Carbohydrates, glycemic index, and pregnancy outcomes in gestational diabetes. Curr. Diab. Rep., 2013. 13(1): p. 6-11.

63. Ma, W.J., Z.H. Huang, B.X. Huang, et al., Intensive low-glycaemic-load dietary intervention for the management of glycaemia and serum lipids among women with gestational diabetes: a randomized control trial. Public Health Nutr., 2015. 18(8): p. 1506-13.

64. Scholl, T.O., X. Chen, C.S. Khoo, et al., The dietary glycemic index during pregnancy: influence on infant birth weight, fetal growth, and biomarkers of carbohydrate metabolism. Am. J. Epidemiol., 2004. 159(5): p. 467-74.

65. Berti, C., H.K. Biesalski, R. Gartner, et al., Micronutrients in pregnancy: current knowledge and unresolved questions. Clin. Nutr., 2011. 30(6): p. 689-701.

66. Heer, M. and S. Egert, Nutrients other than carbohydrates: their effects on glucose homeostasis in humans. Diabetes Metab. Res. Rev., 2015. 31(1): p. 14-35.

67. Moore, W.T., S.M. Bowser, D.W. Fausnacht, et al., Beta Cell Function and the Nutritional State: Dietary Factors that Influence Insulin Secretion. Curr. Diab. Rep., 2015. 15(10): p. 76.

68. Zhang, C. and S. Rawal, Dietary iron intake, iron status, and gestational diabetes. Am. J. Clin. Nutr., 2017.

69. Zhuang, T., H. Han, and Z. Yang, Iron, Oxidative Stress and Gestational Diabetes. Nutrients, 2014. 6(9): p. 3968-3980.

70. Potischman, N., Biologic and methodologic issues for nutritional biomarkers. J. Nutr., 2003. 133 Suppl 3: p. 875S$880 \mathrm{~S}$.

71. Bo, S., A. Lezo, G. Menato, et al., Gestational hyperglycemia, zinc, selenium, and antioxidant vitamins. Nutrition, 2005. 21(2): p. 186-91.

72. Zhang, C., M.A. Williams, I.O. Frederick, et al., Vitamin C and the risk of gestational diabetes mellitus: a case-control study. J. Reprod. Med., 2004. 49(4): p. 257-66.

73. Zhang, C., M.A. Williams, T.K. Sorensen, et al., Maternal plasma ascorbic Acid (vitamin C) and risk of gestational diabetes mellitus. Epidemiology, 2004. 15(5): p. 597-604.

74. Freedman, L.S., P.M. Guenther, K.W. Dodd, et al., The population distribution of ratios of usual intakes of dietary components that are consumed every day can be estimated from repeated 24-hour recalls. J. Nutr., 2010. 140(1): $p$. 111-6.

75. Freedman, L.S., P.M. Guenther, S.M. Krebs-Smith, et al., A population's distribution of Healthy Eating Index-2005 component scores can be estimated when more than one 24-hour recall is available. J. Nutr., 2010. 140(8): p. 152934.

76. Timon, C.M., R. van den Barg, R.J. Blain, et al., A review of the design and validation of web-and computer-based 24-h dietary recall tools. Nutr. Res. Rev., 2016. 29(2): p. 268-280.

77. Hodge, A., A.J. Patterson, W.J. Brown, et al., The Anti Cancer Council of Victoria FFQ: relative validity of nutrient intakes compared with weighed food records in young to middle-aged women in a study of iron supplementation. Aust. N. Z. J. Public Health, 2000. 24(6): p. 576-83.

78. Freedman, L.S., A. Schatzkin, D. Midthune, et al., Dealing with dietary measurement error in nutritional cohort studies. J. Natl. Cancer Inst., 2011. 103(14): p. 1086-92.

79. Meader, N., K. King, T. Moe-Byrne, et al., A systematic review on the clustering and co-occurrence of multiple risk behaviours. BMC Public Health, 2016. 16: p. 657.

80. Noble, N., C. Paul, H. Turon, et al., Which modifiable health risk behaviours are related? A systematic review of the clustering of Smoking, Nutrition, Alcohol and Physical activity ('SNAP') health risk factors. Prev. Med., 2015. 81: p. 16-41.

81. St-Onge, M.P., M.A. Grandner, D. Brown, et al., Sleep Duration and Quality: Impact on Lifestyle Behaviors and Cardiometabolic Health: A Scientific Statement From the American Heart Association. Circulation, 2016. 134(18): $p$. e367-e386.

82. Badon, S.E., D.A. Enquobahrie, P.D. Wartko, et al., Healthy Lifestyle During Early Pregnancy and Risk of Gestational Diabetes Mellitus. Am. J. Epidemiol., 2017. 186(3): p. 326-333.

83. Zhang, C., D.K. Tobias, J.E. Chavarro, et al., Adherence to healthy lifestyle and risk of gestational diabetes mellitus: prospective cohort study. BMJ, 2014. 349: p. g5450.

84. Kim, C., D.K. Berger, and S. Chamany, Recurrence of gestational diabetes mellitus a systematic review. Diabetes Care, 2007. 30(5): p. 1314-1319.

85. Arendz, I.J., P.H. Oomen, A. Wolthuis, et al., [Prevalence of gestational diabetes in high-risk pregnancies: screened using an oral glucose tolerance test]. Ned. Tijdschr. Geneeskd., 2013. 157(18): p. A5409.

86. Barbour, L.A., C.E. McCurdy, T.L. Hernandez, et al., Cellular mechanisms for insulin resistance in normal pregnancy and gestational diabetes. Diabetes Care, 2007. 30 Suppl 2: p. S112-9.

87. Catalano, P.M., Trying to understand gestational diabetes. Diabet. Med., 2014. 31(3): p. 273-81.

88. Schoenaker, D.A., S.S. Soedamah-Muthu, and G.D. Mishra, Quantifying the mediating effect of body mass index on the relation between a Mediterranean diet and development of maternal pregnancy complications: the Australian Longitudinal Study on Women's Health. Am. J. Clin. Nutr., 2016. 104(3): p. 638-45.

89. Markovic, T.P., R. Muirhead, S. Overs, et al., Randomized Controlled Trial Investigating the Effects of a Low-Glycemic Index Diet on Pregnancy Outcomes in Women at High Risk of Gestational Diabetes Mellitus: The GI Baby 3 Study. Diabetes Care, 2016. 39(1): p. 31-38. 
90. Mustila, T., J. Raitanen, P. Keskinen, et al., Pragmatic controlled trial to prevent childhood obesity in maternity and child health care clinics: pregnancy and infant weight outcomes (the VACOPP Study). BMC Pediatr., 2013. 13: p. 80.

91. Thornton, Y.S., C. Smarkola, S.M. Kopacz, et al., Perinatal outcomes in nutritionally monitored obese pregnant women: a randomized clinical trial. J. Natl. Med. Assoc., 2009. 101(6): p. 569-77.

92. Vinter, C.A., D.M. Jensen, P. Ovesen, et al., The LiP (Lifestyle in Pregnancy) study: a randomized controlled trial of lifestyle intervention in 360 obese pregnant women. Diabetes Care, 2011. 34(12): p. 2502-7.

93. Wolff, S., J. Legarth, K. Vangsgaard, et al., A randomized trial of the effects of dietary counseling on gestational weight gain and glucose metabolism in obese pregnant women. Int. J. Obes. (Lond.), 2008. 32(3): p. 495-501.

94. Cuco, G., J. Fernandez-Ballart, J. Sala, et al., Dietary patterns and associated lifestyles in preconception, pregnancy and postpartum. Eur. J. Clin. Nutr., 2006. 60(3): p. 364-71.

95. Gresham, E., C.E. Collins, G.D. Mishra, et al., Diet quality before or during pregnancy and the relationship with pregnancy and birth outcomes: the Australian Longitudinal Study on Women's Health. Public Health Nutr., 2016. 19(16): p. 2975-2983.

96. McGowan, C.A. and F.M. McAuliffe, Maternal dietary patterns and associated nutrient intakes during each trimester of pregnancy. Public Health Nutr., 2013. 16(1): p. 97-107.

97. Shepherd, E., J.C. Gomersall, J. Tieu, et al., Combined diet and exercise interventions for preventing gestational diabetes mellitus. The Cochrane database of systematic reviews, 2017. 11: p. CD010443.

98. Godfrey, K.M., W. Cutfield, S.Y. Chan, et al., Nutritional Intervention Preconception and During Pregnancy to Maintain Healthy Glucose Metabolism and Offspring Health ("NiPPeR"): study protocol for a randomised controlled trial. Trials, 2017. 18(1): p. 131.

99. Blumfield, M.L., A.J. Hure, L. Macdonald-Wicks, et al., Systematic review and meta-analysis of energy and macronutrient intakes during pregnancy in developed countries. Nutr. Rev., 2012. 70(6): p. 322-36.

100. Blumfield, M.L., A.J. Hure, L. Macdonald-Wicks, et al., A systematic review and meta-analysis of micronutrient intakes during pregnancy in developed countries. Nutr. Rev., 2013. 71(2): p. 118-32.

101. Dubois, L., M. Diasparra, B. Bedard, et al., Adequacy of nutritional intake from food and supplements in a cohort of pregnant women in Quebec, Canada: the 3D Cohort Study (Design, Develop, Discover). Am. J. Clin. Nutr., 2017. 106(2): p. 541-548.

102. Livock, M., P.J. Anderson, S. Lewis, et al., Maternal micronutrient consumption periconceptionally and during pregnancy: a prospective cohort study. Public Health Nutr., 2017: p. 1-11.

103. Hure, A., A. Young, R. Smith, et al., Diet and pregnancy status in Australian women. Public Health Nutr., 2009. 12(6): p. 853-861.

104. Goletzke, J., A.E. Buyken, J.C.Y. Louie, et al., Dietary micronutrient intake during pregnancy is a function of carbohydrate quality. Am. J. Clin. Nutr., 2015. 102(3): p. 626-632.

105. Crozier, S.R., S.M. Robinson, K.M. Godfrey, et al., Women's dietary patterns change little from before to during pregnancy. J. Nutr., 2009. 139(10): p. 1956-63.

106. Stephenson, J., D. Patel, G. Barrett, et al., How do women prepare for pregnancy? Preconception experiences of women attending antenatal services and views of health professionals. PLoS One, 2014. 9(7): p. e103085.

107. Szwajcer, E., G.J. Hiddink, L. Maas, et al., Nutrition awareness before and throughout different trimesters in pregnancy: a quantitative study among Dutch women. Fam. Pract., 2012. 29 Suppl 1: p. i82-i88.

108. Blondin, J.H. and J.A. LoGiudice, Pregnant women's knowledge and awareness of nutrition. Appl. Nurs. Res., 2018. 39: p. 167-174.

109. Dean, S.V., Z.S. Lassi, A.M. Imam, et al., Preconception care: closing the gap in the continuum of care to accelerate improvements in maternal, newborn and child health. Reproductive health, 2014. 11 Suppl 3: p. S1.

110. Barker, D.J.P., K.M. Godfrey, P.D. Gluckman, et al., Fetal nutrition and cardiovascular disease in adult life. The Lancet, 1993. 341(8850): p. 938-941.

111. Roseboom, T.J., J.H. van der Meulen, A.C. Ravelli, et al., Effects of prenatal exposure to the Dutch famine on adult disease in later life: an overview. Mol. Cell. Endocrinol., 2001. 185(1-2): p. 93-8.

112. Hammiche, F., J.S. Laven, N. van Mil, et al., Tailored preconceptional dietary and lifestyle counselling in a tertiary outpatient clinic in The Netherlands. Hum. Reprod., 2011. 26(9): p. 2432-41.

113. Homan, G., M. Davies, and R. Norman, The impact of lifestyle factors on reproductive performance in the general population and those undergoing infertility treatment: a review. Hum. Reprod. Update, 2007. 13(3): p. 209-223.

114. Mutsaerts, M.A., H. Groen, N.C. ter Bogt, et al., The LIFESTYLE study: costs and effects of a structured lifestyle program in overweight and obese subfertile women to reduce the need for fertility treatment and improve reproductive outcome. A randomised controlled trial. BMC Womens Health, 2010. 10: p. 22.

115. Muktabhant, B., T.A. Lawrie, P. Lumbiganon, et al., Diet or exercise, or both, for preventing excessive weight gain in pregnancy. The Cochrane database of systematic reviews, 2015(6): p. CD007145.

116. Schoenaker, D.A., S.S. Soedamah-Muthu, and G.D. Mishra, The association between dietary factors and gestational hypertension and pre-eclampsia: a systematic review and meta-analysis of observational studies. BioMed Central Medicine, 2014. 12(1): p. 1.

117. Allen, R., E. Rogozinska, P. Sivarajasingam, et al., Effect of diet-and lifestyle-based metabolic risk-modifying interventions on preeclampsia: a meta-analysis. Acta Obstet. Gynecol. Scand., 2014. 93(10): p. 973-85.

118. Emmett, P.M., L.R. Jones, and J. Golding, Pregnancy diet and associated outcomes in the Avon Longitudinal Study of Parents and Children. Nutr. Rev., 2015. 73 Suppl 3: p. 154-74. 


\section{Chapter 9}

119. Saunders, L., L. Guldner, N. Costet, et al., Effect of a Mediterranean diet during pregnancy on fetal growth and preterm delivery: results from a French Caribbean Mother-Child Cohort Study (TIMOUN). Paediatr. Perinat. Epidemiol., 2014. 28(3): p. 235-44.

120. Brantsæter, A.L., M. Haugen, R. Myhre, et al., Diet matters, particularly in pregnancy: results from MoBa studies of maternal diet and pregnancy outcomes. Norsk Epidemiologi, 2014. 24: p. 63-77.

121. Aronsson, C.A., K. Vehik, J. Yang, et al., Use of dietary supplements in pregnant women in relation to sociodemographic factors - a report from The Environmental Determinants of Diabetes in the Young (TEDDY) study. Public Health Nutr., 2013. 16(8): p. 1390-402.

122. Pouchieu, C., R. Levy, C. Faure, et al., Socioeconomic, lifestyle and dietary factors associated with dietary supplement use during pregnancy. PLoS One, 2013. 8(8): p. e70733.

123. Bassler, K.H., Use and abuse of high dosages of vitamin B6. Int. J. Vitam. Nutr. Res. Suppl., 1989. 30: p. 120-6.

124. Simpson, J.L., L.B. Bailey, K. Pietrzik, et al., Micronutrients and women of reproductive potential: required dietary intake and consequences of dietary deficiency or excess. Part II--vitamin D, vitamin A, iron, zinc, iodine, essential fatty acids. J. Matern. Fetal Neonatal Med., 2011. 24(1): p. 1-24.

125. Valera-Gran, D., M. Garcia de la Hera, E.M. Navarrete-Munoz, et al., Folic acid supplements during pregnancy and child psychomotor development after the first year of life. JAMA pediatrics, 2014. 168(11): p. e142611.

126. NVOG, Diabetes Mellitus en Zwangerschap. Nederlandse Vereniging voor Obstetrie en Gynaecologie, 2010: p. Versie 2.0.

127. Health Council of the Netherlands, Preconception care: a good beginning. Publication no. 2007/19, 2007: p. The Hague: Health Council of the Netherlands.

128. Jungheim, E.S., J.L. Travieso, and M.M. Hopeman, Weighing the impact of obesity on female reproductive function and fertility. Nutr. Rev., 2013. 71 Suppl 1: p. S3-8. 
General discussion 

Summary 
Gestational diabetes mellitus (GDM) is one of the most common metabolic complications during pregnancy and prevalence has continued to increase worldwide. As BMI is one of the most important risk factors for development of GDM, prevention of GDM has focused on modifiable factors including diet. A range of dietary factors has been investigated, but results per dietary factor are limited and inconclusive (chapter 1). This thesis aims to provide more insight into dietary intake and nutrient status before and during pregnancy and into the association of dietary intake and nutrient status with risk of GDM. In addition, two methodological topics are addressed: the development of an index reflecting adherence to the Dutch dietary guidelines of 2015 and the effect of combining food frequency questionnaire (FFQ) and 24-hour recall (24hR) data on bias in diet-disease associations.

In chapter 2, the development and evaluation of the Dutch Healthy Diet 2015 (DHD15) index score is described. The DHD15 index score assesses adherence to the Dutch dietary guidelines of 2015 and consists of 15 components representing the 15 food-based dietary guidelines. Per component the score ranges between zero and ten, resulting in a total score between zero (no adherence) and 150 (complete adherence). We evaluated the DHD15 index based on data from two 24hR and one FFQ from 885 men and women, aged 20-70 years, participating in the longitudinal NQplus study. A higher DHD15 index score was inversely associated with BMI, smoking, and intakes of energy, total fat, and saturated fat and positively associated with energy-adjusted micronutrient intakes. We concluded that the DHD15 index is a good marker of diet quality.

Measurement error in dietary intakes leads to biased diet-disease associations. If dietary intake is assessed with a second method, regression calibration (RC) or enhanced regression calibration (ERC) can be used to combine dietary intakes. This could result in less biased associations. In chapter 3, we investigated the effect of combining dietary intakes obtained with two methods (24hR and FFQ) on diet-disease associations using data from 236 participants of the NQplus study. We observed that combining FFQ and $24 \mathrm{hR}$ data with RC and ERC for protein and potassium resulted in empirical attenuation factors approaching 1 , indicating less biased diet-disease associations.

In chapter 4, the prevalence of common delivery-related adverse pregnancy outcomes was compared between women with normal glucose tolerance during pregnancy (NGT), women with treated GDM and women with untreated borderline GDM (BGDM). BGDM was defined as women with an abnormal 50-grams glucose challenge test and a normal 75-grams oral glucose tolerance test. Medical file data were used from 1049 women who had undergone GDM screening and had given birth in hospital Gelderse Vallei Ede in the period 2010-2014. Women with treated GDM had a higher risk of laceration and women with untreated BGDM had a higher risk of laceration and (unscheduled) Caesarean section as compared to women with NGT. Furthermore, we observed that associations between glucose 
tolerance and adverse pregnancy outcomes were not mediated by macrosomia (birth weight $>4000$ gram).

In chapter 5, the associations between pre-pregnancy dietary carbohydrate quantity and quality with risk of GDM were investigated in women of reproductive age in an Australian cohort. A total of 3,607 women aged 25-30 years were followed-up for self-reported GDM development from 2003 till 2015. Pre-pregnancy dietary intake was assessed with a FFQ in 2003 and 2009. Carbohydrate quantity was assessed based on total carbohydrate intake and the low-carbohydrate diet (LCD) score. Carbohydrate quality was assessed by studying fibre intake, total sugar intake, glycaemic index, glycaemic load and intake of carbohydrate-rich food groups. A relatively low carbohydrate and high fat and protein intake as expressed by the LCD score and a higher cereal intake were significantly associated with higher risk of GDM, whereas higher fibre, fruit and fruit juice intakes were associated with lower risk of GDM.

Chapter 6 describes the prevalence of inadequate micronutrient intakes and the association of prepregnancy micronutrient adequacy with risk of GDM in the same Australian cohort of women of reproductive age. We used a summary measure of adequate micronutrient intake across 13 micronutrients, i.e. the mean adequacy ratio (MAR). Prevalence of inadequate dietary micronutrient intake was more than $50 \%$ for the micronutrients calcium, potassium, magnesium, vitamin $\mathrm{E}$ and folate, indicating suboptimal pre-pregnancy micronutrient intakes. A higher overall micronutrient adequacy (i.e. MAR) was associated with a lower risk of developing GDM.

In chapter 7, changes in folate, vitamin B6, vitamin B12, vitamin D and iron intake, their status markers and diet quality from preconception to the second trimester of pregnancy are described. In addition, associations of these micronutrient intakes, their status markers and diet quality with glucose tolerance during pregnancy were examined. Data from 91 Dutch women at increased risk of GDM, aged 18-40 years, and with either a wish to get pregnant or less than 24 weeks pregnant was collected longitudinally. Data was collected at preconception $(n=67), 12$ weeks of pregnancy $(n=47)$ and 24 weeks of pregnancy $(n=55)$. At each time point women underwent a fasting venipuncture and a 75grams oral glucose tolerance test. Dietary intake was assessed at each time point with a validated FFQ and two non-consecutive $24 \mathrm{hR}$. We observed significant changes in total micronutrient intakes throughout pregnancy, due to changes in supplemental intakes, whereas dietary micronutrient intakes and diet quality remained stable. Nutrient status levels changed significantly from preconception to the second trimester of pregnancy. For folate, vitamin B6 and vitamin D this could be partly explained by changes in intake. In general, no associations between fasting and 2-hour glucose levels and HbA1c levels with diet quality, micronutrient intake or status levels were observed; except for a weak inverse association of folate intake with 2-hour glucose levels, and a weak positive association between ferritin 
and 2-hour glucose levels. Diet quality was inversely associated with fasting glucose in a sensitivity analysis, excluding data from participants with only one measurement.

Using data from the same study collected at preconception from the same study $(n=66)$, main dietary sources of folate, vitamin $D$, and the $n-3$ fatty acids eicosapentaenoic acid (EPA) and docosahexaenoic acid (DHA) were determined in chapter 8. Additionally, associations of these intakes with their blood levels were examined. A large proportion of women did not meet the Dutch recommended intakes of folate $(50 \%)$, vitamin D (67\%), and EPA+DHA (52\%). Vegetables were the main contributor of dietary folate intake (25\%), oils and fats of dietary vitamin D intake (39\%), and fish of dietary EPA and DHA intake (69\%). Fourteen percent had an inadequate folate status and $23 \%$ an inadequate vitamin $D$ status. Supplemental folate intake, supplemental and dietary vitamin D intake and dietary EPA+DHA intake were significantly associated with their blood levels.

In chapter 9, the main findings of this thesis were summarized and a reflection on methodological aspects was given. Considering results described in this thesis and associations reported by other studies, it is probable that a healthy diet can reduce risk of developing GDM. Most convincing evidence was observed for diet in the pre-pregnancy period. More research is needed to understand which foods and nutrients drive diet-GDM associations, and to unravel underlying mechanisms. Diet in the pre-pregnancy period was considered suboptimal and intakes of several vital micronutrients, including folate, were below recommended intakes for a large proportion of women in two different studies. Although the Health Council of the Netherlands already called upon the provision of proper and adequate preconception care a decade ago, more action is needed to improve intake and status of essential micronutrients in the preconception period. 
Summary 

Dutch Summary

Nederlandse samenvatting 
Diabetes gravidarum (ook wel zwangerschapsdiabetes of gestational diabetes genoemd) is één van de meest voorkomende metabole complicaties die tijdens de zwangerschap kan optreden. We spreken van diabetes gravidarum (GDM) als er sprake is van koolhydratenintolerantie of een hoge bloedsuiker (glucose) spiegel tijdens de zwangerschap. Het hebben van overgewicht of obesitas is één van de grootste risicofactoren voor het krijgen van GDM. Daarom richt de preventie van GDM zich op risicofactoren van obesitas die we kunnen veranderen, zoals de voedingsinname. In Hoofdstuk 1 wordt een overzicht gegeven van onderzoek naar voedingsinname en GDM. Helaas zijn de resultaten beperkt en niet altijd eenduidig. Het doel van dit proefschrift is om meer inzicht te krijgen in de voedingsinname en nutriëntstatus van vrouwen voor en tijdens hun zwangerschap en de associatie tussen voedingsinname, nutriëntstatus en het risico op het krijgen van GDM. Daarnaast bekijken we twee methodologische aspecten van het meten van voedingsinname: 1) het ontwikkelen van een score die weergeeft hoe goed iemand eet volgens de Nederlandse voedingsrichtlijnen van 2015 en 2) het combineren van voedingsinnamegegevens verzameld met een voedselfrequentievragenlijst (FFQ) met voedingsinnamegegevens verzameld met een 24 -uursnavraag (24hR) en effect daarvan op voedingsziekte associaties.

In Hoofdstuk 2 wordt de ontwikkeling en evaluatie van de Dutch Healthy Diet 2015 (DHD15) index score beschreven. Deze DHD15 index score geeft weer in welke mate iemand voldoet aan de Nederlandse voedingsrichtlijnen van 2015. De index score bestaat uit 15 componenten, 1 voor elke richtlijn. Voor elke component kan een score tussen de 0 en 10 punten behaald worden. Deze componentscores worden bij elkaar opgeteld, wat resulteert in de totaalscore. De laagste totaalscore van 0 punten geeft aan dat iemand helemaal niet voldoet aan de richtlijnen en de hoogste totaalscore van 150 score wordt gegeven als iemand voldoet aan alle richtlijnen. Om de DHD15 index score te evalueren hebben we de gegevens gebruikt van 885 mannen en vrouwen van tussen de 20 en 70 jaar oud die hebben meegedaan aan de longitudinale NQplus studie en twee 24hR en één FFQ hebben ingevuld. We zagen dat een hogere DHD15 index score negatief geassocieerd was met BMI, roken en energie-, vet-, en verzadigd vetinname en positief geassocieerd was met micronutriëntinnames nadat deze gecorrigeerd waren voor energie-inname. We concluderen hieruit dat de DHD15 index een goede graadmeter is voor het bepalen van de kwaliteit van de voeding.

Meetfouten in voedingsinnamegegevens kan leiden tot systematische fouten in gerapporteerde voedings-ziekte associaties. Indien de voedingsinname met een tweede methode wordt gemeten, kan de regressiecalibratie (RC) of uitgebreide regressiecalibratie (ERC) techniek gebruikt worden om de voedingsinnamegegevens van de twee verschillende methodes te combineren. Het combineren van voedingsinnamegegevens van twee verschillende methoden kan leiden tot minder fouten in voedingsziekte associaties. In Hoofdstuk $\mathbf{3}$ onderzoeken we wat het effect is van het combineren van 
voedingsinnamegegevens verzameld met twee verschillende methodes (24hR en FFQ) op de systematische fout in voedings-ziekte associaties. Hiervoor gebruikten we gegevens van 236 deelnemers van de NQplus studie. We zagen dat het combineren (met behulp van de RC en ERC technieken) van FFQ en $24 \mathrm{hR}$ innamegegevens van eiwit en kalium leidde tot attenuatiefactoren dichtbij 1. Dit geeft aan dat het combineren van FFQ en $24 \mathrm{hR}$ gegevens kan leiden tot minder systematische fout in gerapporteerde voedings-ziekte associaties.

In Hoofdstuk 4 hebben we veelvoorkomende bevallingscomplicaties vergeleken tussen vrouwen met normale glucosetolerantie (NGT) tijdens de zwangerschap, vrouwen met GDM die behandeld werden en vrouwen met licht afwijkende glucose-intolerantie, maar die geen behandeling ondergingen (BGDM). Voor dit onderzoek hebben we medische gegevens van 1049 vrouwen gebruikt. Deze vrouwen waren getest op GDM en zijn bevallen in ziekenhuis Gelderse Vallei tussen 2010 en 2014. Vrouwen met behandelde GDM hadden een hoger risico op een ruptuur, terwijl vrouwen met onbehandelde BGDM een hoger risico hadden op een ruptuur en (ongeplande) keizersnede vergeleken met vrouwen met NGT. Verder zagen we dat de associaties tussen glucosetolerantie en bevallingscomplicaties niet gemedieerd werden door macrosomie (geboortegewicht $>4000 \mathrm{~g}$ ).

In Hoofdstuk 5 bekeken we of de hoeveelheid en kwaliteit van de koolhydraten in de voeding voor de zwangerschap geassocieerd was met het risico op het krijgen van GDM. Hiervoor gebruikten we gegevens van 3607 Australische vrouwen tussen de 25 en 30 jaar oud die 12 jaar lang (2003-2015) gevolgd werden om te kijken of ze GDM kregen. Voedingsinname voor de zwangerschap werd in 2003 en 2009 gemeten met een FFQ. De koolhydratenhoeveelheid werd gemeten aan de hand van totaal gegeten koolhydraten en de low-carbohydate diet (LCD) score. Koolhydratenkwaliteit werd bekeken met behulp van de totale hoeveelheid vezelinname, suikerinname, glycemische index, glycemische load en inname van koolhydraatrijke voedselgroepen. Een relatief lage koolhydrateninname en hoge vet en eiwitinname (weergegeven met de LCD-score) en een hoge inname van ontbijtgranen was geassocieerd met een hoger risico op het ontwikkelen van GDM, terwijl een hoge vezelinname, fruitinname en vruchtensapinname geassocieerd waren met een lager risico op het krijgen van GDM.

In Hoofdstuk 6 beschrijven we de prevalentie van inadequate micronutriëntinname en de associatie met het krijgen van zwangerschapsdiabetes. Hiervoor gebruikten we de gegevens van dezelfde 3607 Australische vrouwen. We gebruiken de mean adequacy ratio (MAR) om weer te geven of de micronutriëntinname van 13 verschillende micronutriënten adequaat is. Meer dan $50 \%$ van de vrouwen had onvoldoende inname van calcium, kalium, magnesium, vitamine $E$ en foliumzuur. Vrouwen met een meer adequate micronutriëntinname (weergegeven met de MAR), hadden een lager risico om GDM te ontwikkelen. 
In Hoofdstuk 7 wordt beschreven hoe de inname van foliumzuur, vitamine B6, vitamine B12, vitamine D en ijzer, hun statusmarkers en kwaliteit van de voeding veranderen van de preconceptiefase tot het tweede trimester van de zwangerschap. Daarnaast worden de associaties van deze micronutriëntinnames, statusmarkers en kwaliteit van de voeding met glucosetolerantie tijdens de zwangerschap beschreven. Hiervoor gebruikten we longitudinale gegevens van 91 Nederlandse vrouwen (18-40 jaar oud) met een verhoogd risico op GDM die of een zwangerschapswens hadden of minder dan 24 weken zwanger waren. Gegevens werden verzameld voor de zwangerschap ( $n=67)$, bij 12 weken zwangerschap ( $n=47$ ) en bij 24 weken zwangerschap ( $n=55)$. Op elk meetmoment was er een nuchtere bloedafname en een 75grams glucosetolerantie test. Voedingsinname werd op elk meetmoment gemeten met een gevalideerde FFQ en $24 \mathrm{hR}$ op twee willekeurige, niet-opeenvolgende dagen. We zagen dat de micronutriëntinnames significant veranderden tijdens de zwangerschap door veranderingen in supplementinname. Micronutriëntinnames uit de voeding bleef gelijk. Ook de nutriëntstatus veranderde significant tijdens de zwangerschap. De veranderingen in nutriëntstatus van foliumzuur, vitamine B6 en vitamine D konden gedeeltelijk verklaard worden door veranderingen in de inname. Over het algemeen zagen we geen associatie tussen nuchtere glucose, 2-uurs glucose en HbA1c waardes met kwaliteit van de voeding, micronutriëntinname of micronutriëntstatus. De enige uitzonderingen waren een zwakke negatieve associatie tussen foliumzuur en 2-uurs glucose en een zwakke positieve associatie tussen ijzerstatus en 2-uurs glucosewaardes. Kwaliteit van de voeding was negatief geassocieerd met nuchtere glucosewaardes in een sensitiviteitsanalyse, waarin de gegevens van deelnemers die maar één meting hadden gedaan niet mee werden genomen.

Met de gegevens van dezelfde studie hebben we in Hoofdstuk 8 gekeken naar de belangrijkste voedingsbronnen van foliumzuur, vitamine $D$ en de omega-3-vetzuren EPA en DHA in de preconceptiefase $(n=66)$. Daarnaast hebben we gekeken of deze micronutriëntinnames geassocieerd waren met micronutriëntbloedwaardes. Een groot deel van de vrouwen kreeg minder binnen dan de aanbevolen hoeveelheden voor foliumzuur (50\%), vitamine D (67\%) en EPA+DHA (52\%). Groenten waren de belangrijkste voedingsbron van foliumzuurinname $(25 \%)$, oliën en vetten van vitamine $D$ (39\%) en vis van EPA en DHA (69\%). Veertien procent van de vrouwen had een inadequate foliumzuurstatus en $23 \%$ had een inadequate vitamine D-status. Foliumzuurinname uit supplementen, vitamine D-inname uit zowel de voeding als supplementen en EPA+DHA-inname uit de voeding waren significant geassocieerd met bloedwaardes van deze micronutriënten.

In Hoofdstuk 9 worden de belangrijkste bevindingen van dit proefschrift samengevat en worden de belangrijkste methodologische aspecten besproken. Wanneer we zowel de resultaten van dit promotieonderzoek als de resultaten van overige onderzoeken beschouwen, kan er geconcludeerd worden dat gezonde voeding het risico op het ontwikkelen van GDM waarschijnlijk kan verminderen. 
Het meest overtuigende bewijs daarvoor komt uit onderzoeken die hebben gekeken naar voedingsinname voor de zwangerschap. $\mathrm{Er}$ is meer onderzoek nodig om te begrijpen welke voedingsmiddelen en nutriënten ten grondslag liggen aan deze waargenomen associaties en welke mechanismes hierbij een rol spelen. De voedingsinname in de periode voor de zwangerschap bleek suboptimaal en de innames van verschillende micronutriënten, waaronder foliumzuur, bleken voor een groot deel van de vrouwen uit twee verschillende studies lager te zijn dan de aanbevolen dagelijkse hoeveelheid. Hoewel de Gezondheidsraad al meer dan 10 jaar geleden opriep tot een betere preconceptiezorg, is er nog steeds veel te verbeteren wat betreft de inname en nutriëntstatus van essentiële micronutriënten in de preconceptiefase. 

Acknowledgements

Dankwoord 
Zo, dat was het: vier jaar werk samengevat in 200 pagina's. Natuurlijk heb ik al het werk niet alleen gedaan en waren er bloed, zweet, tranen en heel veel andere mensen voor nodig om dit voor elkaar te krijgen. Al die mensen wil ik hierbij ontzettend bedanken en een paar mensen in het bijzonder.

Allereerst mijn co-promotor Anouk en promotor Edith. Ontzettend bedankt dat jullie het aandurfden om met mij deze uitdaging aan te gaan. We wisten dat de studie uitdagend zou worden en dankzij jullie verloor ik nooit de hoop op een goede afloop. Anouk, ik kon altijd bij jou terecht met vragen en twijfels. Ik heb ontzettend veel van je geleerd en ik wil je onwijs bedanken voor de fijne samenwerking. Edith, zonder jouw positieve instelling, eeuwige optimisme, creatieve ideeën en vertrouwen was ik niet zover gekomen, bedankt!

Ik wil ook alle co-auteurs van mijn artikelen bedanken. Ontzettend bedankt voor jullie tijd en moeite om de artikelen naar een hoger plan te tillen. In het bijzonder wil ik Daniëlle en Sabita bedanken. Jullie hebben me op het idee gebracht om naar Australië te gaan om met de ALSWH data te werken, en jullie waren onwijs betrokken bij het tot een goed einde brengen van de twee ALSWH artikelen. Gita and colleagues of the Centre for Longitudinal and Life Course Research, thank you for the opportunities, hospitality, lunches, coffee breaks and board game nights during my amazing two month stay in Australia.

I would like to thank the committee Bas Kemp, Tessa Roseboom, Harold de Valk and Maijalliisa Erkkola for reading my thesis and being present at my defence.

Het grootste gedeelte van de afgelopen vier jaar heb ik gewerkt aan het opzetten en uitvoeren van de GLIMP-studies. Dat was een enorm leerzame ervaring en was nooit gelukt zonder de hulp van heel veel mensen. Allereerst wil ik Rik en Rahul bedanken die van het begin af aan bij het project betroken zijn geweest en mij enorm op weg hebben geholpen in de wondere wereld van ziekenhuizen. Verder wil ik iedereen van Ziekenhuis Gelderse Vallei, Rijnstate en Slingeland Ziekenhuis bedanken voor hun inzet voor de GLIMP-studies.

Ook alle deelnemers van de GLIMP-studies hartelijk bedankt voor uw bijdrage. Zonder $\mathrm{u}$ is er geen onderzoek mogelijk!

Ook vanuit de afdeling Humane Voeding hebben ontzettend veel mensen een steentje bijgedragen aan de opzet en uitvoer van de GLIMP-studies. ledereen die ik de afgelopen jaren hiervoor heb lastiggevallen, ontzettend bedankt. Ik ben bijzonder veel dank verschuldigd aan Anne, die mijn go-to persoon was bij elke vraag die ik had en veel logistiek en administratief (denk)werk uit handen heeft genomen. Ook onderzoeksmedewerkers Noortje bij de start van de GLIMP2 studie en Renske en Ellen bij het laatste jaar van de studie, zonder jullie inzet was het nooit gelukt, ontzettend bedankt! 
Very, Malou, Nina, Marinda, Eline, Vera, Hannah, Isobel, Ellen, Claudia, Paulie, Isabel, Anne, ik vond het erg leuk om jullie te begeleiden bij jullie thesis of stage. Bedankt voor jullie tomeloze inzet bij de metingen.

Eén ding is zeker, zonder de steun van de fantastische collega's van de afdeling had ik het niet gered. Bedankt voor de vaak nuttige en altijd gezellige koffiepauzes, eerst in het Agro en later met de HNEfavorieten in het Futurum en Helix. PhDtour commissie 2015 (Janne, Yfke, Irene, Guido, Yuri en Merel), wat was het leuk om met jullie de reis naar de oostkust van de VS te organiseren. Briljante ideeën met een soms wat tegenvallende praktijk hebben gezorgd voor onvergetelijke herinneringen. Marije, wat vond ik het fijn om een vaste kantoorgenoot te krijgen die ook net begonnen was met haar PhD. Samen hebben we heel wat dingen uitgevogeld en dankzij jou leerde ik dat ook grote projecten met meerdere partners zijn nadelen had. Je was een onmisbare steun en inspireerde me op mindere dagen, superfijn dat je me nu wil bijstaan als paranimf! Yfke, bij jou kon ik altijd mijn hart luchten, even afleiding zoeken of een nodige schop onder m'n kont krijgen. Samen hebben we de oostkust van de VS onveilig gemaakt scheurend in onze mini-van en heerlijk geborreld (o-nee, genetwerkt) op zonnige terrasjes. Heel erg leuk dat je me wil bijstaan als paranimf.

Gelukkig waren er ook genoeg mensen om me te aan herinneren aan een leven naast het werk. Lieve meiden van de Restancie, bedankt voor alle leuke etentjes en weekendjes weg. Ilse en Marleen, sinds de middelbare school hebben we al een hoop meegemaakt. Bedankt voor alle gezelligheid, uitjes en heerlijke onzin op zijn tijd!

Waar zou ik zijn zonder mijn lieve (schoon)familie. Ed, Ank en Mandy, bedankt voor de lieve mensen die jullie zijn. Jeroen, onwijs lieve, grappige en soms zeer irritante broer. Bedankt voor jouw nuchterheid, heerlijke serieuze en minder serieuze gesprekken. Sas, jouw zwangerschap en de geboorte van Lynn gaven me een andere kijk op mijn onderzoek. Ik kan met jou heerlijk shoppen, kletsen of genieten van een lekker stuk taart. Je hebt altijd een luisterend oor en bent de beste zus die ik me kan wensen. Pap en mam, zonder jullie was ik nergens, bedankt voor jullie onvoorwaardelijke steun!

Last, maar zeker not least: Mikel. Mijn denker, mijn doener en alles er tussenin op de momenten dat ik het zelf allemaal niet meer wist. Het (lees ik) was zeker niet altijd even makkelijk. Bedankt dat je altijd in me gelooft, zelfs als ik onverwacht reisjes naar de VS en Australië aankondig. Zonder jouw steun, liefheid en begrip was het me niet gelukt.

\section{Moniek}



About the author 
CV

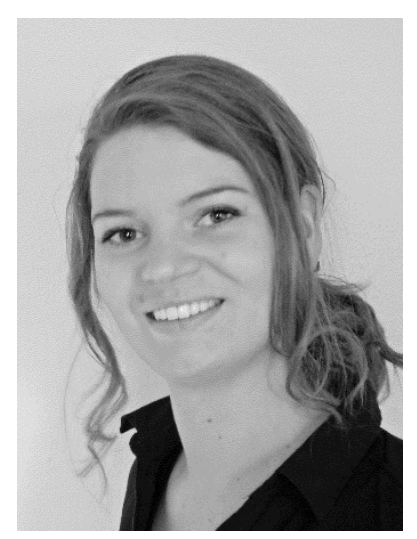

Moniek Looman was born on February $20^{\text {th }}, 1990$ in Zutphen, the Netherlands. In 2008, she received her secondary school diploma from Stedelijk Dalton College in Zutphen. Shortly thereafter, she started her postsecondary education in Nutrition and Health at Wageningen University. She did her MSc thesis within the SLIMMER project, a diabetes prevention program implemented in a real-life setting. She looked at the effect of a ten-month lifestyle intervention on weight, dietary intake and physical activity. She completed her internship at the National Institute of Public Health and the Environment in which she investigated the association of lifestyle changes on cardiovascular risk and all-cause mortality in the Doetinchem Cohort Study. In 2011, Moniek finished her MSc Nutrition and Health, specialization Epidemiology and Public Health cum laude and was appointed as PhD candidate at the Division of Human Nutrition. Her research focussed on the role of diet and nutrient status in the development of gestational diabetes. During her PhD project, Moniek conducted a medical file study and a small-scaled multicentre observational study. In 2017, Moniek visited the School of Public Health of the University of Queensland in Australia to work with data from the ALSWH study and learn more about longitudinal data-analyses and life course research. During her PhD, Moniek joined the educational programme of the graduate school VLAG. She attended several (inter)national conferences and courses and was involved in teaching and supervising BSc and MSc students during their thesis projects. Furthermore, Moniek was member of the organising committee of the academic excursion for fellow PhD candidates to the East Coast of the USA in 2015. In 2017, she was runner-up for the Foppe ten Hoor award during the Dutch Nutritional Science Days. Currently, Moniek is involved in teaching and coordinating Epidemiology and Public Health courses at Wageningen University. 


\section{List of publications}

\section{Publications in peer-reviewed journals}

Looman M, Schoenaker DAJM, Soedamah-Muthu SS, Geelen A, Feskens EJM, Mishra GD. Prepregnancy dietary carbohydrate quantity and quality, and risk of developing gestational diabetes: the Australian Longitudinal Study on Women's Health. Accepted for publication in Br J Nutr

Looman M, Feskens EJM, de Rijk M, Meijboom S, Biesbroek S, Temme EH, de Vries J, Geelen A (2017) Development and evaluation of the Dutch Healthy Diet index 2015. Public Health Nutr 20, 22892299.

Biesbroek S, Verschuren WMM, Boer JMA, van de Kamp ME, van der Schouw YT, Geelen A, Looman M, Temme EHM (2017) Does a better adherence to dietary guidelines reduce mortality risk and environmental impact in the Dutch sub-cohort of the European Prospective Investigation into Cancer and Nutrition? Br J Nutr 118, 69-80.

Hulsegge G, Looman M, Smit HA, Daviglus ML, van der Schouw YT, Verschuren WM (2016) Lifestyle Changes in Young Adulthood and Middle Age and Risk of Cardiovascular Disease and All-Cause Mortality: The Doetinchem Cohort Study. Journal of the American Heart Association 5.

\section{Submitted publications}

Looman M, Boshuizen HC, Feskens, EJM, Geelen A. Using enhanced regression calibration to combine dietary intake estimates from 24-hour recall and food frequency questionnaire improves diet-disease associations. Submitted.

Looman M, Geelen A, Zandstra D, Samlal RAK, Heijligenberg R, Feskens EJM. Adverse pregnancy outcomes in treated gestational diabetes and untreated borderline gestational diabetes. Submitted.

Looman M, Schoenaker DAJM, Soedamah-Muthu SS, Mishra GD Geelen A, Feskens EJM. Prepregnancy dietary micronutrient adequacy and risk of developing gestational diabetes: the Australian Longitudinal Study on Women's Health. Submitted.

Looman M, van den Berg C, Geelen A, Samlal RAK, Heijligenberg R, Klein Gunnewiek JMT, Balvers MGJ, Leendertz-Eggen CL, Wijnberger LDE, Feskens EJM, Brouwer-Brolsma EM. Supplement use and dietary sources of folate, vitamin $\mathrm{D}$, and $\mathrm{n}-3$ fatty acids during preconception: the GLIMP2 study. Submitted. 
About the author

\section{Published abstracts}

Looman M, Geelen A, Samlal RAK, Heijligenberg R, Feskens EJM (2015) Gestational diabetes mellitus and macrosomia in a Dutch population: determinants and other adverse pregnancy outcomes. Eur J Epidemiol 30,787-788

Looman M, Schoenaker DAJM, Soedamah-Muthu SS, Geelen A, Feskens EJM, Mishra GD (2017) Prepregnancy dietary, lifestyle and reproductive risk factors of gestational diabetes in an Australian population-based cohort. Journal Dev Orig Health Dis 8, S387-S388. 


\section{Overview of completed training activities}

Discipline specific activities

Organiser and location

Year

\section{Courses}

Good Clinical Practice cursus

Exposure assessment

Masterclass confounding

Epigenetics and epigenesis

Multivariate analysis for food data/sciences

Conferences and meetings

Food for Thought

Wetenschapsavond ZGV

Nationale diabetes dag

Nutritional Science Days

European Congress of Epidemiology

- Healthy Living

Diabetes and pregnancy screening and prevention meeting

WEON

World Congress Developmental Origins of Health and Disease

\section{Presentations}

Oral presentation, Food for Thought

Oral presentation, Nutritional Science Days

Oral presentation, Nutritional Science Days

Poster presentation, European Congress of Epidemiology

Poster presentation, WEON

Poster presentation, World Congress DOHaD
Ziekenhuis Gelderse Vallei, Ede 2014

VLAG, Wageningen 2014

VLAG, Wageningen 2014

VLAG, Wageningen 2014

VLAG, Wageningen 2016

Alliantie Voeding in de Zorg, Ede 2013-2017 Ziekenhuis Gelderse Vallei, Ede 2014-2016 NDF, Den Haag 2014 NWO, Heeze

VvE, Maastricht 2015

DALI project group, Brussels 2015 VvE, Wageningen 2016

DOHaD Society, Rotterdam 2017

Alliantie Voeding in de Zorg, Ede 2015 NWO, Heeze 2015

NWO, Heeze 2017

VvE, Maastricht2 2015

VvE, Wageningen 2016

DOHaD Society, Rotterdam 
About the author

\section{General courses}

PhD week

VLAG, Wageningen

2014

Data Management

WGS, Wageningen

2014

Scientific Publishing

WGS, Wageningen

2016

Scientific Writing

WGS, Wageningen

2015

Project and Time management

WGS, Wageningen

2015

Teaching and Supervising thesis students

ESD, Wageningen

2015

Career perspectives

WGS, Wageningen

2017

Optional courses and activities

Preparation of PhD proposal

HNE/VLAG, Wageningen

2014

PhD study tour USA

HNE/VLAG, Wageningen

2015

Organizing Committee PhD study tour 2015

HNE/VLAG, Wageningen

2014-2015

MENU-D research meetings

HNE, Wageningen

2015-2018

Paperclip research meetings

HNE, Wageningen

2014-2018 
Notes 


\section{Colophon}

The research described in this thesis was financially supported by the Division of Human Nutrition, Wageningen University.

Financial support from Wageningen University for printing this thesis is gratefully acknowledged.

Cover Mikel Minkman

Lay-out Moniek Looman

Printed by Digiforce - Proefschriftmaken.nl, Vianen

Copyright (C) Moniek Looman, 2018 



\section{Propositions}

1. Gestational diabetes prevention should focus on improving diet quality before as well as during pregnancy (this thesis)

2. Despite publichealth campaigns, micronutrient intakes of women in the preconception period remain inadequate (this thesis)

3. Nonceliac gluten sensitivity can be explained by a nocebo effecti.e. induction or worsening of symptoms due to negative expectations

4. Risk avoidance is a favourable personality trait

5. Small is beautiful also applies to research

6. Coffee breaks are essential for a PhD student

7. Every thinker needs a doer, and vice-versa

Propositions belonging to the thesis entitled:

Diet, nutrient status and gestational diabetes

Moniek Looman

Wageningen, May 18th 2018 